\title{
Magnetic Phases in the Hubbard Model
}

\author{
Dissertation \\ zur Erlangung des mathematisch-naturwissenschaftlichen \\ Doktorgrades \\ "Doctor rerum naturalium" \\ der Georg-August-Universität Göttingen
}

vorgelegt von

Robert Peters

aus Karl-Marx-Stadt (jetzt Chemnitz)

Göttingen, 2009 
D 7

Referent: Prof. Dr. Thomas Pruschke Koreferent: Prof. Dr. Frithjof Anders

Tag der mündlichen Prüfung : 19.11.2009 
<smiles>C#CC#CC#CC#CC(C)(C#C)C#C</smiles> 
Lines from "Hsin-hsin Ming"

Shinjinmei (or Shinjin no Mei) (Japanese)

By Third Ch'an Patriarch Chien-chih Seng-ts'an

"Kon ni ki sureba shi o e. Shõ ni shitagaeba shû o shissu." 


\section{Contents}

1 Magnetism and Strong Correlations $\quad 7$

1.1 Introduction . . . . . . . . . . . . . . . . 7

1.2 Transition Metal Oxides . . . . . . . . . . . . . 8

1.2.1 Light Transition Metal Oxides . . . . . . . . . . 11

1.2 .2 Cobaltates . . . . . . . . . . . . . . . . 12

1.2.3 Cuprates ....................... 13

1.2.4 Manganites . . . . . . . . . . . . . . . . . . 14

1.3 Hubbard Model . . . . . . . . . . . . . . . . . . . . . 17

1.4 Exchange Interactions . . . . . . . . . . . . . . 20

1.5 Types of Magnetic Order . . . . . . . . . . . . . . . 22

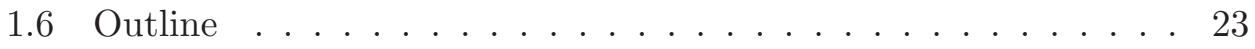

2 Dynamical Mean Field Theory 25

2.1 Introduction . . . . . . . . . . . . . . . . . 25

2.2 Cavity Construction . . . . . . . . . . . . . . 25

2.3 Dynamical Mean Field Theory . . . . . . . . . . . . . . 28

2.4 Magnetic solutions . . . . . . . . . . . . . . . . . . 30

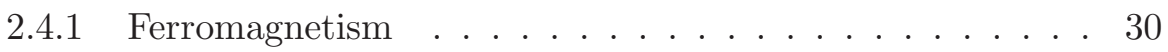

2.4 .2 Antiferromagnetism . . . . . . . . . . . . 32

2.5 Bethe lattice . . . . . . . . . . . . . . . . . . . 32

2.5.1 Bethe lattice with next nearest neighbor hopping . . . . 33

3 Impurity Solver - Theoretical Background 39

3.1 The Anderson Impurity Model . . . . . . . . . . . . . . . . . . . 39

3.2 Discretization of the Band States . . . . . . . . . . . . . 41

3.3 Numerical Renormalization Group . . . . . . . . . . . . . 42

3.3.1 Discretization within the NRG . . . . . . . . . 42

3.3.2 Iterative diagonalization . . . . . . . . . . . . . 43

3.3.3 Calculation of impurity properties . . . . . . . . . 45 
3.3.4 Calculating the Self-energy . . . . . . . . . . . 48

3.3.5 Two-orbital Anderson model . . . . . . . . . . . . . . 49

3.4 Density Matrix Renormalization Group . . . . . . . . . . . 50

3.4 .1 Iterative diagonalization . . . . . . . . . . 50

3.4.2 Calculation of dynamical properties . . . . . . . 52

3.4 .3 Algorithm . . . . . . . . . . . . . . . 53

4 NRG and DMRG Spectral Functions for the Impurity Model 55

4.1 Introduction . . . . . . . . . . . . . . . . . 55

4.2 Used Discretization Schemes . . . . . . . . . . . . . . 57

4.3 Deconvolution . . . . . . . . . . . . . . . . . 58

4.3 .1 Matrix Inversion . . . . . . . . . . . . . . 58

4.3.2 Maximum Entropy Ansatz . . . . . . . . . . . . . . . 59

4.4 Comparison between DMRG and NRG . . . . . . . . . . . 60

4.4 .1 Single Impurity Anderson Model . . . . . . . . . . . . . 60

4.4 .2 Two-Orbital Anderson Model . . . . . . . . . . . . . 64

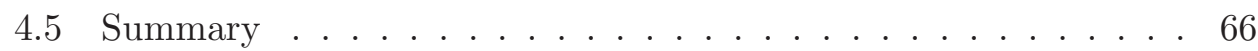

5 One-orbital Hubbard model $\quad 67$

5.1 Introduction . . . . . . . . . . . . . . . . 67

5.2 Semi-elliptic Density of States . . . . . . . . . . . . . . 68

5.2.1 Metal Insulator Transition . . . . . . . . . . . . . 68

5.2 .2 Magnetic Phase Diagram . . . . . . . . . . . . . . 69

5.2 .3 Spin Density Waves . . . . . . . . . . . . . . 72

5.3 Comparison of DMRG and NRG . . . . . . . . . . . 77

5.4 Frustrated Bethe Lattice . . . . . . . . . . . . . . . . . 80

5.4 Half-Filling . . . . . . . . . . . . . . . 80

5.4 .2 Antiferromagnetism at half-filling . . . . . . . . . 82

5.4.3 Nearly fully frustrated system . . . . . . . . . . . . . 85

5.4 .4 Doped System . . . . . . . . . . . . . . 89

5.4.5 Ferromagnetism in the Frustrated system . . . . . . . . 92

5.4.6 Energies for the different magnetic states . . . . . . . . 95

5.5 Summary . . . . . . . . . . . . . . . . . 97

6 Two-Orbital Hubbard Model $\quad 99$

6.1 Introduction . . . . . . . . . . . . . . . . . . . . 999

6.2 Two Site Cluster . . . . . . . . . . . . . . . . . . . 100

6.3 Magnetic Phase Diagram . . . . . . . . . . . . . . . . 103

6.4 Quarter-Filling ....................... 107

6.4.1 Ferromagnetic Metal Insulator Transition . . . . . . . . . 107

6.4.2 Antiferromagnetism and Charge Order . . . . . . . . . . 113

6.5 Summary . . . . . . . . . . . . . . . . 115

$\begin{array}{lll}7 & \text { Summary and Outlook } & 117\end{array}$

$\begin{array}{ll}\text { Appendices } & 121\end{array}$

A Calculation of the Ground State Energy 123 
CHAPTER 1

\section{Magnetism and Strong Correlations}

\subsection{Introduction}

The ability of some materials to attract others has fascinated many people for over 2500 years. Perhaps the widest known legend is by Pliny the elder, who described how the shepherd "Magnes" stuck with his iron nails in his shoes to a black stone while he pastured his flocks. Besides this legend the first definite statements were by Thales of Miletus about 585 B.C., saying that lodestone, the naturally occurring mineral magnetite $\mathrm{Fe}_{3} \mathrm{O}_{4}$, can attract iron [1]. The name magnetite most probably comes from the old Greek city "Magnesia" in Asia Minor, where large depositions of the magnetic mineral could be found $[1,2]$. Since then there have been developed many explanations and applications of the phenomenon magnetism. The first widely known application is the compass, which was most likely invented in China and was then used since the 11th century in Europe [1,2]. William Gilbert, who was an English physicist and natural scientist, wrote in 1600 "De Magnete" [3], in which he describes experiments and phenomenology of magnets and also states that the earth itself is a big magnet explaining the compass. This was the first modern attempt to understand magnetism. In the 19th century a big breakthrough was achieved by many scientists ${ }^{1}$, who noticed and explained the connection between electricity and magnetism [4]. But still the origin of remanent magnetism could not be explained. It was not before the development of the quantum theory of materials [5] and the discovery of the spin in the first half of the 20th century that the way was free for explaining and describing the source of magnetism. The application of magnetic fields is today indispensable. What began with the

\footnotetext{
${ }^{1}$ to mention some of them: C Oersted, J Biot, F Savart, A Ampere, M Faraday and J Maxwell
} 
compass, is nowadays used in power production, means of transportation and computer devices like storage and memory. Today's magnetic materials used in electronics are not some stones found in nature, but precisely engineered thin films and multilayer structures on the nanometer scale [6]. The guiding principles today are "smaller and faster". Thus the exact study and understanding of the underlying physics in the nowadays used materials and structures is crucial for designing new devices.

Today's theories about the origin of magnetism rely on the concepts of charge, spin and quantum mechanics developed in the early 20th century [5]. The pioneers of the quantum theory noticed that the angular momentum and the spin of electrons can form magnetic moments, which eventually align. This arrangement of moments is the origin of the magnetic fields observed around permanent magnets [1]. The widely known magnetism often actually denotes ferromagnetism in which all magnetic moments are aligned parallel. But in general it is clear that the moments may align in more complicated arrangements, if this is energetically favorable. In this work I will use the term "magnetic ordering" in the sense of some periodic, long range alignment of spins regardless of the definite arrangement.

The Curie temperature of iron, the temperature below which the magnetic moments are aligned parallel, is 1043K [7]. One should now ask, what is the force, which is responsible for this order. From a classical electrodynamics lecture [4], one knows that magnetic moments act on each other via a dipole interaction. One can simply estimate the strength of this interaction in iron to be $0.1 \mathrm{~K}[7]$. For sure, this force is too weak to produce such a high Curie temperature. As it will become clear below, the reasons for the alignment are actually the Coulomb interaction between electrons, and the Pauli exclusion principle.

If one looks at the periodic table of elements and searches for the elements, which show ferromagnetic behavior at room temperature one will only find $\mathrm{Fe}$, Co, Ni. All three have partially filled d-orbitals. When looking for chemical compounds, which order at room temperature or below, one must notice that often partially filled d- or f-orbitals are involved. So there are three major groups showing magnetic ordering: transition metals, transition metal oxides and rare earth elements as well as actinides and their compounds [7]. Besides these groups one should also mention that some organic compounds including transition metal atoms can show magnetic ordering [7].

\subsection{Transition Metal Oxides}

The above discussion results in the obvious question: How is magnetic ordering connected to d-orbitals? Consider the $3 \mathrm{~d}$-orbital atomic wave function in a transition metal. The wave function of a free atom can always be written as a product of a radial part and a spherical harmonics. The $3 \mathrm{~d}$-orbitals have angular momentum $l=2$. As the $1 s-, 2 s-, 2 p-, 3 s-$ and $3 p$-orbitals have different angular momentum, the $3 \mathrm{~d}$-orbitals are orthogonal to them because of their spherical harmonics. Thus the radial part of the wave function has 


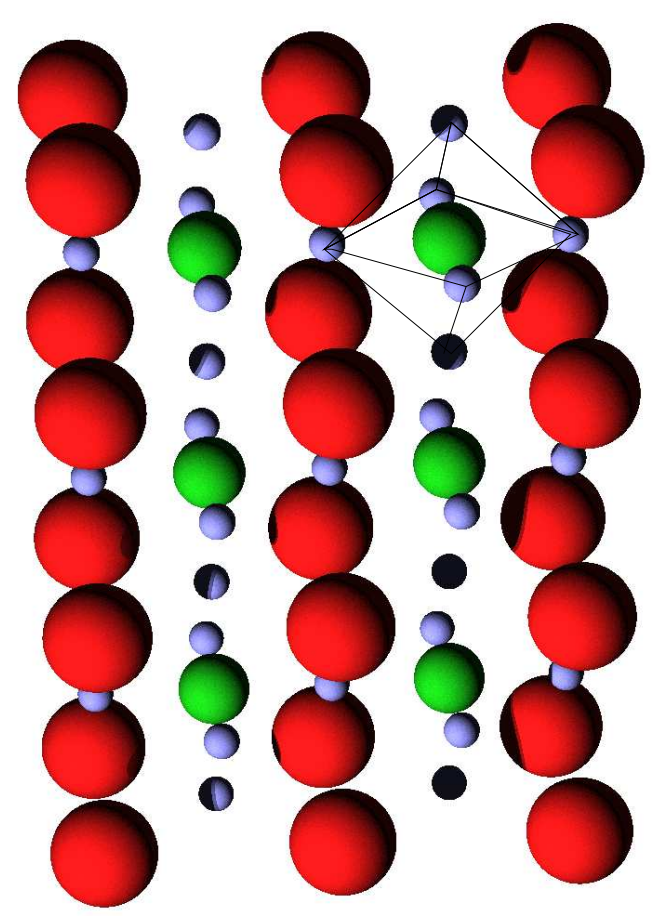

Figure 1.1: Cubic perovskite structure of a typical transition metal oxide. The green spheres are the transition metal atoms $(\mathrm{M})$, which are surrounded by octahedra of oxygen drawn as small blue spheres. The big red spheres represent the A-atoms of $\mathrm{AMO}_{3}$.

no node and does not "extend" as far as the 3s- or 3p-orbitals. In contrast, the 4 s-orbitals with angular momentum $l=0$ extend very far from the nucleus of the atoms. The 4s-orbitals lie energetically lower than the $3 \mathrm{~d}$-orbitals and are filled before them. The electrons in the 4s-bands are most important for screening the Coulomb interaction in the $3 \mathrm{~d}$-band $[8,9]$. When the energy difference between both bands is large, the screening is very poor leading to a strong Coulomb interaction between the d-electrons. On the other hand, the 3d-orbitals extend less and thus have only a small overlap with each other. Therefore the bandwidth is much smaller, resulting in a tendency to localize, which can lead to more pronounced correlation effects.

The following elements are most important when studying the physics of $3 \mathrm{~d}-$ transition metals: Ti, V, Cr, Mn, Fe, Co, Ni and Cu. These elements often form compounds with oxygen [10]. The typical structure of transition metal oxides is the cubic perovskite like $\mathrm{AMO}_{3}$, see figure 1.1, where the transition metal atoms $(\mathrm{M})$, green spheres, are surrounded by an octahedra of oxygen atoms $(\mathrm{O})$, blue spheres. The A-atoms, red spheres, represent some heavy elements. These compounds can contain partially filled d-shells, which turn out to be especially important for explaining their physical properties. The transition metal atoms are situated in an octahedral environment. Crystal field theory predicts in this case, that the 5 -fold degenerate d-orbitals split into 3 -fold $t_{2 g}$-levels and 2 -fold $e_{g}$-levels [7]. In figure 1.2 one can see the level splitting of d-orbitals in 


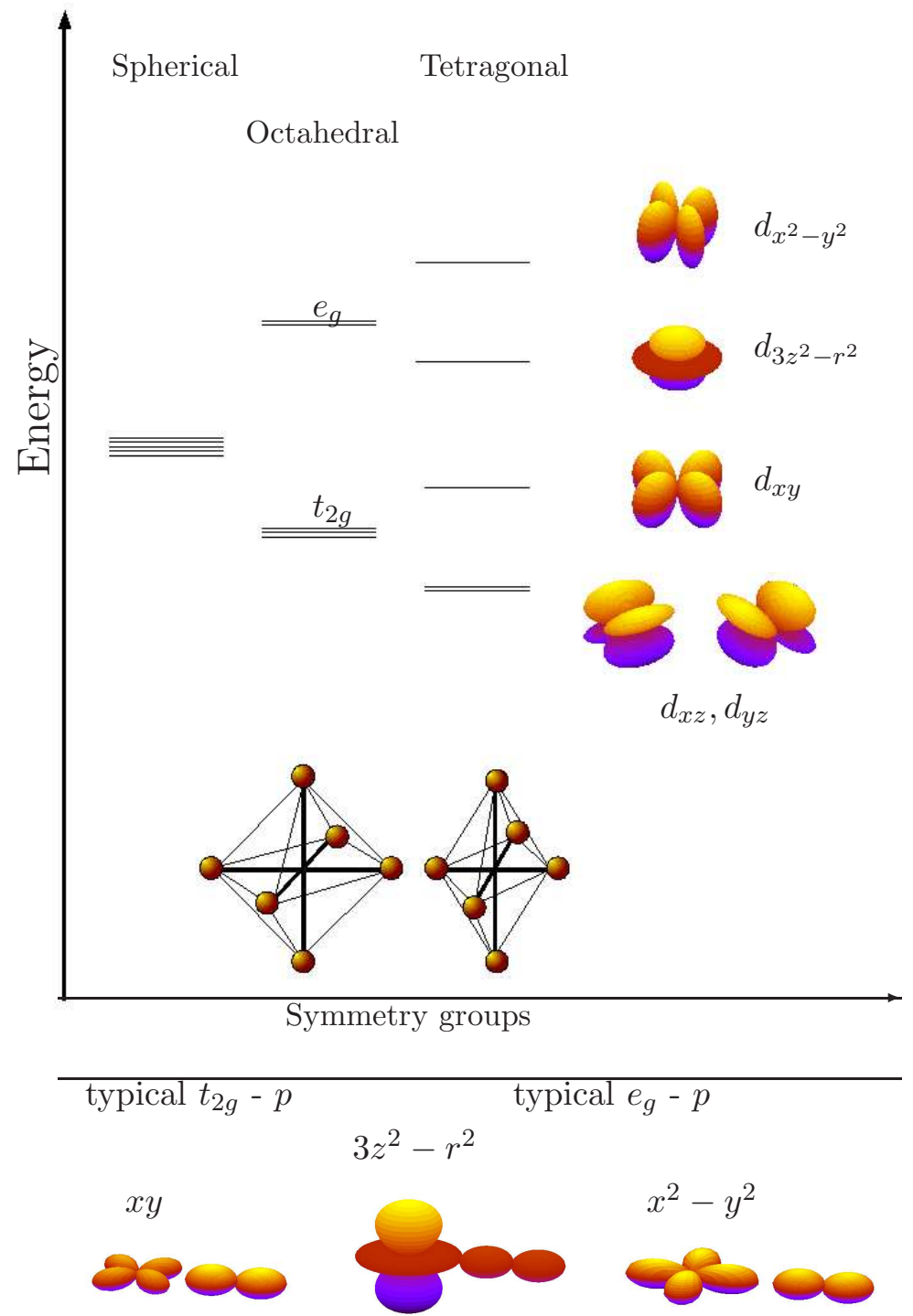

Figure 1.2: Energetic splitting of the d-orbitals in different symmetry groups. The energy is in arbitrary units, as the picture is only supposed to illustrate the splitting. The exact position of the levels depend on the specific system. Left: 5-fold degenerate levels in spherical symmetry. Next: Splitting in octahedral symmetry into a $t_{2 g^{-}}$and $e_{g}$-band. The $t_{2 g}$-levels have lower energy in a cubic perovskite. Next: Splitting in tetragonal symmetry, as it occurs in a static Jahn-Teller distortion [11]. Right: Shape and classification of the d-orbitals. The spread in "z"-direction is color coded. The pictures at the bottom show typical configurations between one $\mathrm{d}$ - and a neighboring p-orbital showing that the overlap is largest for the $e_{g}$-levels. 
octahedral and tetrahedral environment. On the right side of the figure one can see the basic shape of the d-orbitals. In the cubic perovskite the orbitals of the $t_{2 g}$-wave functions point to the corners of the cube while the $e_{g}$-wave functions point to the faces of the cube where the oxygen atoms are located. In this configuration the $t_{2 g}$-levels are lower in energy due to the repulsive electrostatic interaction with the occupied p-orbitals of oxygen. The splitting of $t_{2 g^{-}}$and $e_{g}$-orbitals can be as large as $2-3 \mathrm{eV}$ [10]. In transition metal oxides the direct overlap between the d-orbitals of neighboring sites is often negligible. The hopping of the electrons from one transition metal atom to another is mediated by the oxygen surrounding these [10]. The strength of this process depends on the exact positions and the energy levels of the oxygen and the transition metal atoms, but it can be assumed to be small. From this fact it is clear that the electrical resistivity of these compounds can show interesting properties. Band theory predicts that a partially filled band is always a metal, but it was already reported in 1937 by J. de Boer and E. Verwey $[12,13]$ that many transition metal oxides with partially filled d-orbitals are very poor metals or even insulators [14]. In a very simplified picture one can imagine that at low temperatures in a halffilled band one electron sits at each lattice site. If now an electron moves through the lattice, it has to pay an extra energy when it visits a site where an electron is already located. For strong enough repulsive interaction, this will prevent the electrons from moving at all [13]. This is an example of a Mott insulator, which is due to strong repulsive electron-electron interactions. An insulator formed by a partially filled shell will have localized electrons, which may form magnetic moments due to their spin and angular momentum. As this localization is a collective phenomenon of the electrons there are correlations between them eventually aligning the magnetic moments. This shows the close connection between strong correlations, metal insulator transition and magnetism. In the following I will address some particular interesting examples of transition metal oxides. Some of the phenomena discussed in the next paragraphs will appear again later in this thesis.

\subsubsection{Light Transition Metal Oxides}

A prominent example showing a metal insulator transition is Vanadiumoxide, $\mathrm{V}_{2} \mathrm{O}_{3}$ [14,15], for with the phase diagram is depicted in figure 1.3. For low temperatures and low pressure an antiferromagnetically ordered state is realized. Applying pressure does not change the local electron-electron interaction in this compound, but it changes the overlap between the electronic wave functions thus changing the bandwidth. Therefore the ratio between the interaction and the bandwidth is tuned. Strikingly, for moderate temperatures a paramagnetic metal insulator transition, a drop in the resistivity of several orders of magnitude, can be observed. The transition can be triggered by changing the pressure, temperature or chemical substitution. Vanadiumoxide crystallizes in the corundum-structure, which introduces frustration to the system [14]. Frustration means here that there are competing magnetic interactions, which cannot all be satisfied simultaneously. This effect can be the reason, why the antiferromagnetic state in $\mathrm{V}_{2} \mathrm{O}_{3}$ becomes quickly unstable, when increasing the 


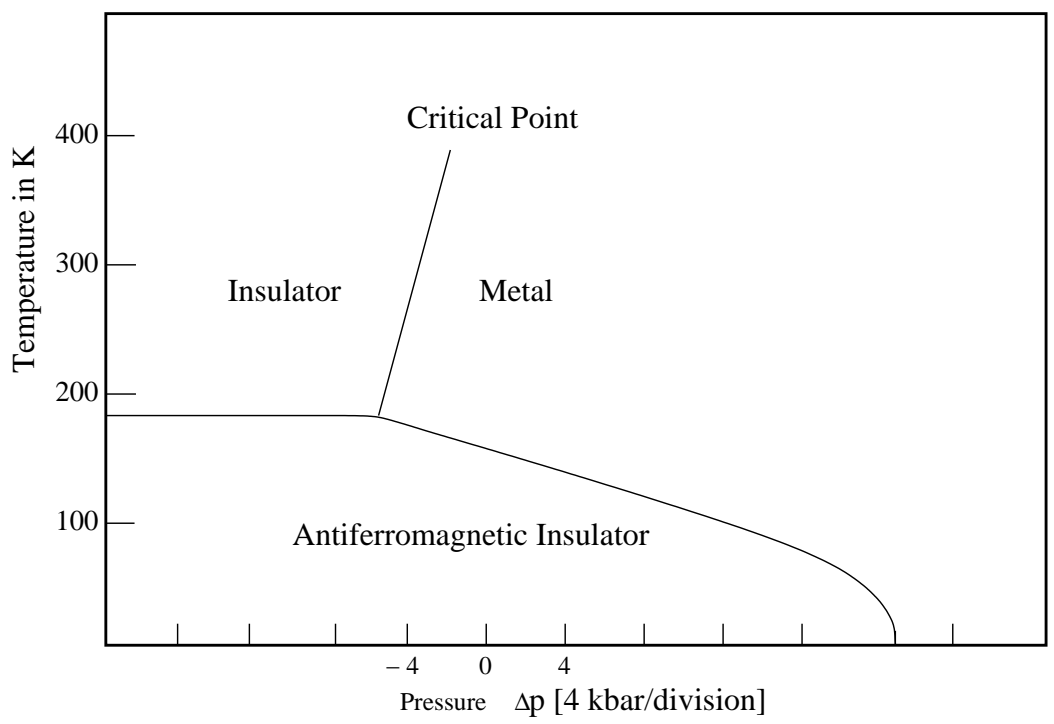

Figure 1.3: Phase diagram of $\mathrm{V}_{2} \mathrm{O}_{3}$ redrawn after D. McWhan et al. [15]. For low temperatures the system forms an antiferromagnetic insulator which becomes unstable towards a paramagnetic metal with increasing pressure. Interestingly, for temperatures between $200 \mathrm{~K}<T<400 \mathrm{~K}$ a pressure dependent paramagnetic metal insulator transition is observed.

temperature and a paramagnetic metal insulator transition can be observed. Other light transition metal oxides with perovskite structure are, for example, $\mathrm{R}_{1-x} \mathrm{~A}_{x} \mathrm{TiO}_{3}$ and $\mathrm{R}_{1-x} \mathrm{~A}_{x} \mathrm{VO}_{3}$ (R: trivalent cations, A: divalent cations) with their parent compounds like $\mathrm{YTiO}_{3}$ and $\mathrm{LaVO}_{3}$. In these substances the $t_{2 g}$-bands are filled with one or two electrons respectively, thus being partially filled. Nevertheless both materials are insulators [16,17]. They are appropriate systems for experimental investigation of the paramagnetic metal insulator transition triggered by doping [18]. For example, with increasing hole concentration in $\mathrm{La}_{1-x} \mathrm{Sr}_{x} \mathrm{TiO}_{3}$, one can observe a metal insulator transition for $x=0.05$.

\subsubsection{Cobaltates}

Cobaltates have been studied because of their quite unique magnetic and transport properties. For example, $\mathrm{NaCO}_{2} \mathrm{O}_{4}$ shows a large thermoelectric effect [19]. A thermoelectric device creates a voltage, when there is a temperature gradient present in the material. The discussed applications are manifold, for example utilization of waste heat or power supply for deep space probes [20]. The dorbitals in Cobaltates are filled with five to six electrons [10,14]. There is a strong competition between different electron configurations, so-called valencefluctuations [21]. At low temperatures all electrons occupy the energetically lower $t_{2 g}$-band forming a low spin configuration. But it is also possible that one or two electrons occupy the $e_{g}$-band forming intermediate or high-spin configurations. The competition between these states lead to very interesting 


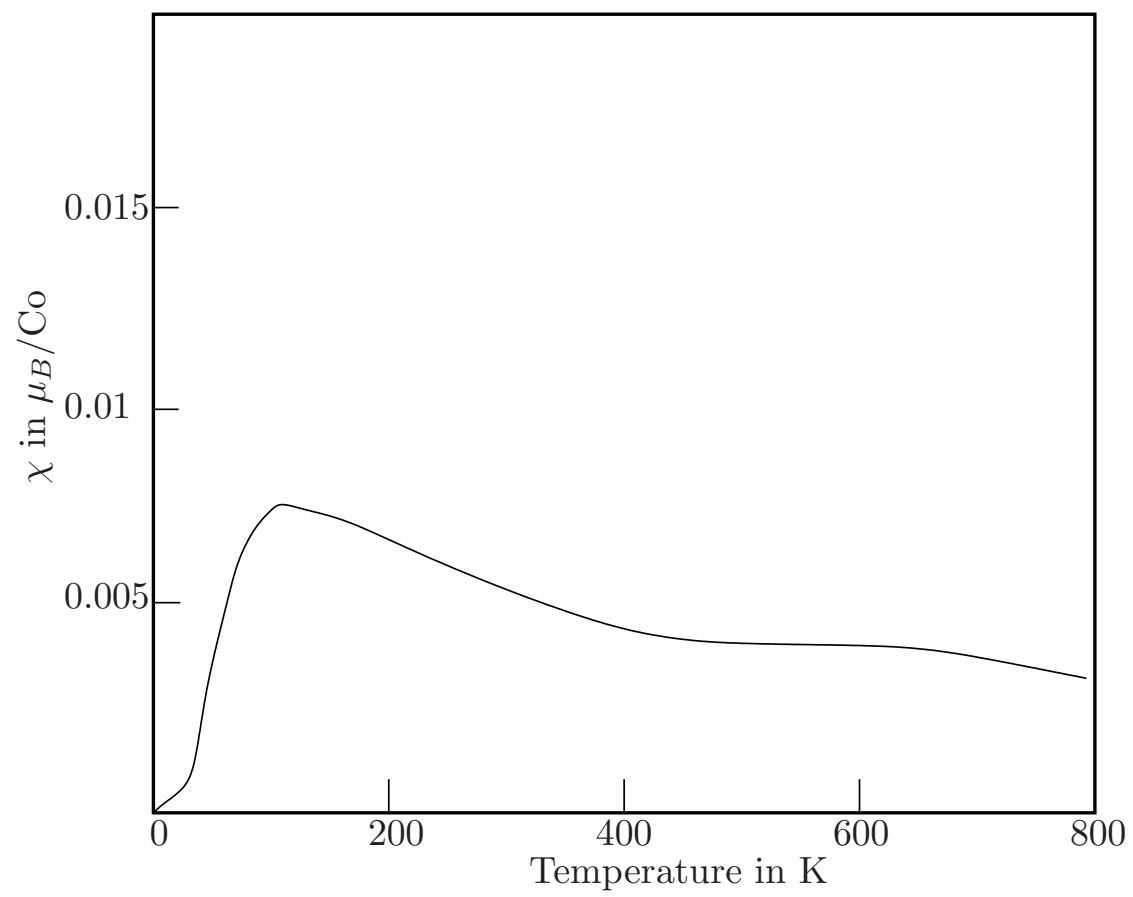

Figure 1.4: Magnetic susceptibility $\chi$ versus temperature of $\mathrm{LaCoO}_{3}$ showing the nonmagnetic ground state and the transition to the high spin state, indicated by the maximum in the curve. (This is a sketch redrawn after M. Imada et al. Rev. Mod. Phys. 701039 (page 1235) [14])

transport and magnetic behavior of $\mathrm{LaCoO}_{3}$ shown in figure 1.4 [14,22,23]. At low temperatures this compound is a non-magnetic insulator with low-spin state meaning the $t_{2 g}$-band is fully occupied. However, for increasing temperature the spin state changes and the magnetic susceptibility shows a maximum at about $100 \mathrm{~K}$. $\mathrm{LaCoO}_{3}$ and the hole-doped $\mathrm{La}_{1-x} \mathrm{Sr}_{x} \mathrm{CoO}_{3}$ are excellent examples how the different orbitals can influence the physics.

\subsubsection{Cuprates}

High-temperature (high- $T_{C}$ ) superconductivity was first discovered in 1986 in the copper oxide $\mathrm{La}_{1.85} \mathrm{Ba}_{0.15} \mathrm{CuO}_{4}[24,25]$. High- $T_{C}$ superconductivity refers to compounds with transition temperatures $T_{C}>30 \mathrm{~K}$ and is a very active field in physics. Today a large number of cuprate compounds showing high- $T_{C}$ superconductivity are known. Their structure is typically a layered perovskite structure with $\mathrm{CuO}_{2}$ layers $[14,25]$. Soon after the discovery it was evident that strong electron correlations are important in these compounds [25, 26]. $\mathrm{La}_{2} \mathrm{CuO}_{4}$ is an antiferromagnetic insulator with a Néel-temperature around $T_{N}=300 \mathrm{~K}$. The degeneracy of the $e_{g}$-orbitals is lifted due to the anisotropy, which mainly originates from the two-dimensionality. $\mathrm{La}_{2} \mathrm{CuO}_{4}$ has 9 electrons

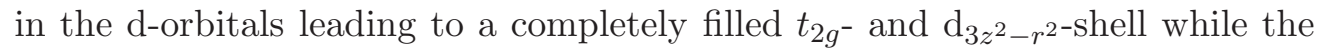
remaining $\mathrm{d}_{x^{2}-y^{2}}$-shell is half-filled. The half-filled band combined with the insulating behavior is a strong evidence for the importance of strong electron 
correlations. The superconductivity arises when a small amount of hole-carriers are introduced by doping for example with $\mathrm{Ba}$ [25]. For some compounds the transition temperature can be higher than $T=77 \mathrm{~K}$, the boiling point of nitrogen $[27]$.

\subsubsection{Manganites}

The last interesting material class which I want to introduce here, are the manganites [14,28-31]. They became famous because of the colossal magneto resistance effect. This is a large change in resistivity upon changing the strength of the magnetic field near the Curie temperature. If one now considers, that reading and writing of memory in a computer or other electronic devices use exactly such resistance changes, one can easily imagine, why these materials receives so much interest. Manganites are composed of $\mathrm{MnO}_{3}$ octahedra with perovskite structure. The manganese d-orbitals have no direct overlap with each other. The overlap is mediated by the inbetween lying oxygen atoms. A typical example for a manganite compound is $\mathrm{La}_{1-x} \mathrm{Ca}_{x} \mathrm{MnO}_{3}$. Figure 1.5 shows a sample picture and some particular important experiments. All three pictures correspond to $\mathrm{La}_{0.75} \mathrm{Ca}_{0.25} \mathrm{MnO}_{3}$. The experiments were performed at the University of Göttingen by S. A. Köster and V. Moshnyaga. The upper left picture shows a photograph of such a sample, while the upper right picture shows a high resolution transmission electron microscopy image of this compound, which was grown on $\mathrm{MgO}$. In this picture a superstructure of $\mathrm{La}$ and Ca was found. Both upper pictures are supposed to give the reader an impression how the samples look like, for which the experiments are performed. The lower pictures show the results of three typical experiments. The resistivity is plotted versus the temperature. For zero magnetic field, the resistivity increases for decreasing temperatures until the sample reaches its Curie temperature. At the Curie temperature there is a sudden drop in the resistivity. By applying a magnetic field of $H=5 \mathrm{~T}$ one can increase the Curie temperature. The resistivity of the system shows a much smaller initial increase, but now decreases at higher temperatures. This change is called the colossal magneto resistance effect [29], as the resistivity around the Curie temperatures changes several order of magnitude. The lower panels show the ferromagnetic magnetization of this compound for low temperatures and a ferromagnetic hysteresis curve, when applying a magnetic field. Such phase transitions into magnetic phases will be the principal focus of this work.

Additionally, the phase diagram of this compound is shown in figure 1.6. The manganese d-orbitals are filled with $n=4-x$ electrons. The electronic configuration is such that strong electron-electron interactions including strong ferromagnetic Hund's coupling play an important role $[33,34]$. Three of the $4-x$ electrons occupy the $t_{2 g}$-orbitals. Ferromagnetic Hund's coupling forces them to align forming a $S=3 / 2$-spin. As the exchange between the $t_{2 g}$-states of different manganese atoms is negligible, these states can be treated as localized spins, which couple ferromagnetically to the $e_{g}$-band. The remaining electrons occupy the $e_{g}$-band. There is a hopping of the $e_{g}$-electrons between different manganese atoms. Similar as in $\mathrm{V}_{2} \mathrm{O}_{3}$, the screening is not very efficient 

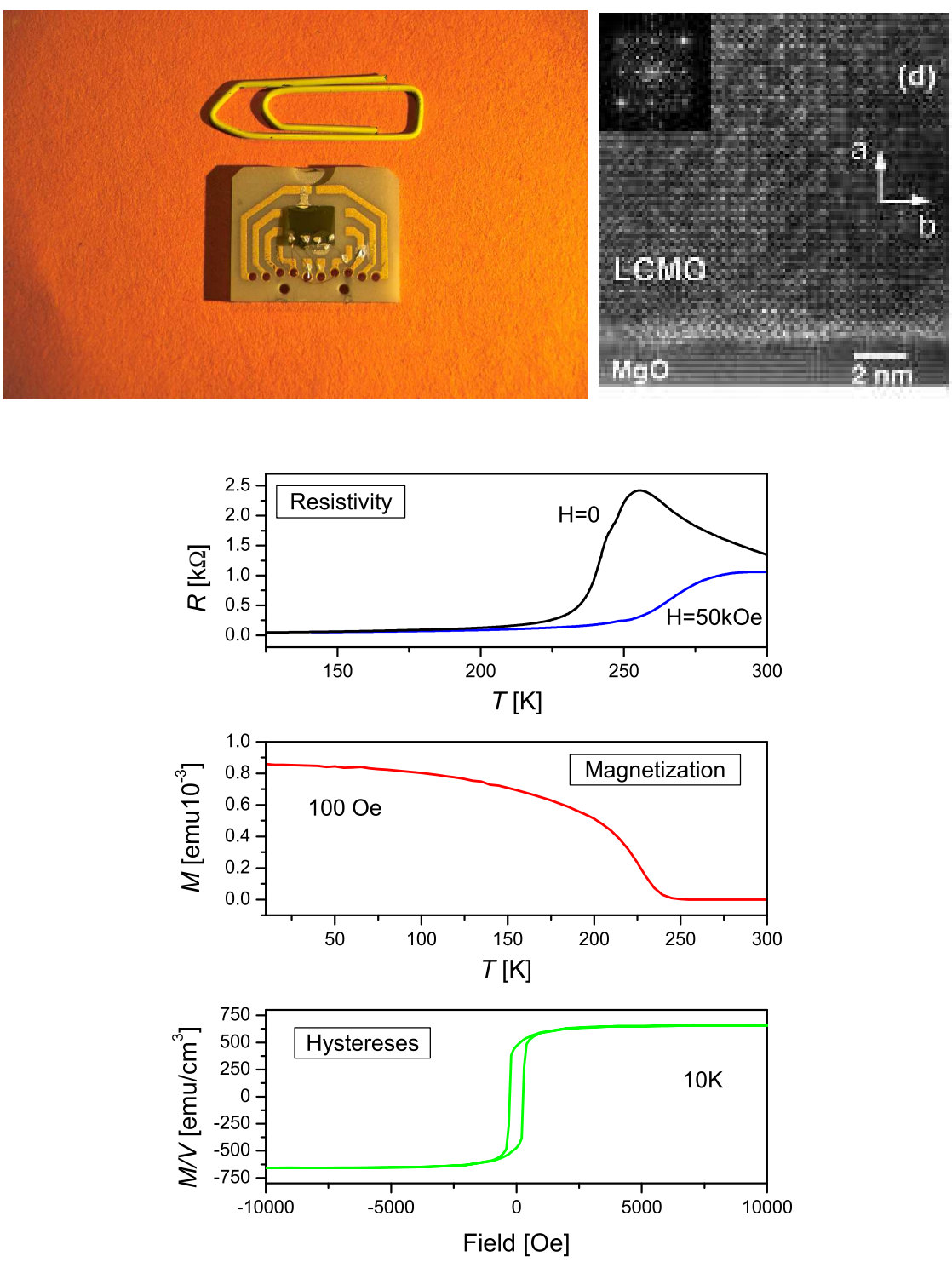

Figure 1.5: All three pictures correspond to $\mathrm{La}_{0.75} \mathrm{Ca}_{0.25} \mathrm{MnO}_{3}$. Top Left: Picture of a sample, taken by S. A. Köster at the University of Göttingen. Top Right: High Resolution Transmission Electron Microscopy image of such a sample. The $\mathrm{La}_{0.75} \mathrm{Ca}_{0.25} \mathrm{MnO}_{3}$ thin film is grown on $\mathrm{MgO}$. Remarkably, V. Moshnyaga et al. found a perovskite superstructure due to La/Ca ordering. (Reprinted with permission from V. Moshnyaga et al. Phys. Rev. Lett. 97, 107205 (2006). COPYRIGHT 2006 by American Physical Society [32]) Bottom: The picture shows three experiments, performed by S. A. Köster and V. Moshnyaga, in the ferromagnetic region of this compound. The upper panel shows the resistance $R$ for two different magnetic fields $H$. The middle panel shows the ferromagnetic magnetization $M$ via temperature $T$ and the lower panel shows a ferromagnetic hysteresis curve $M(H)$. 


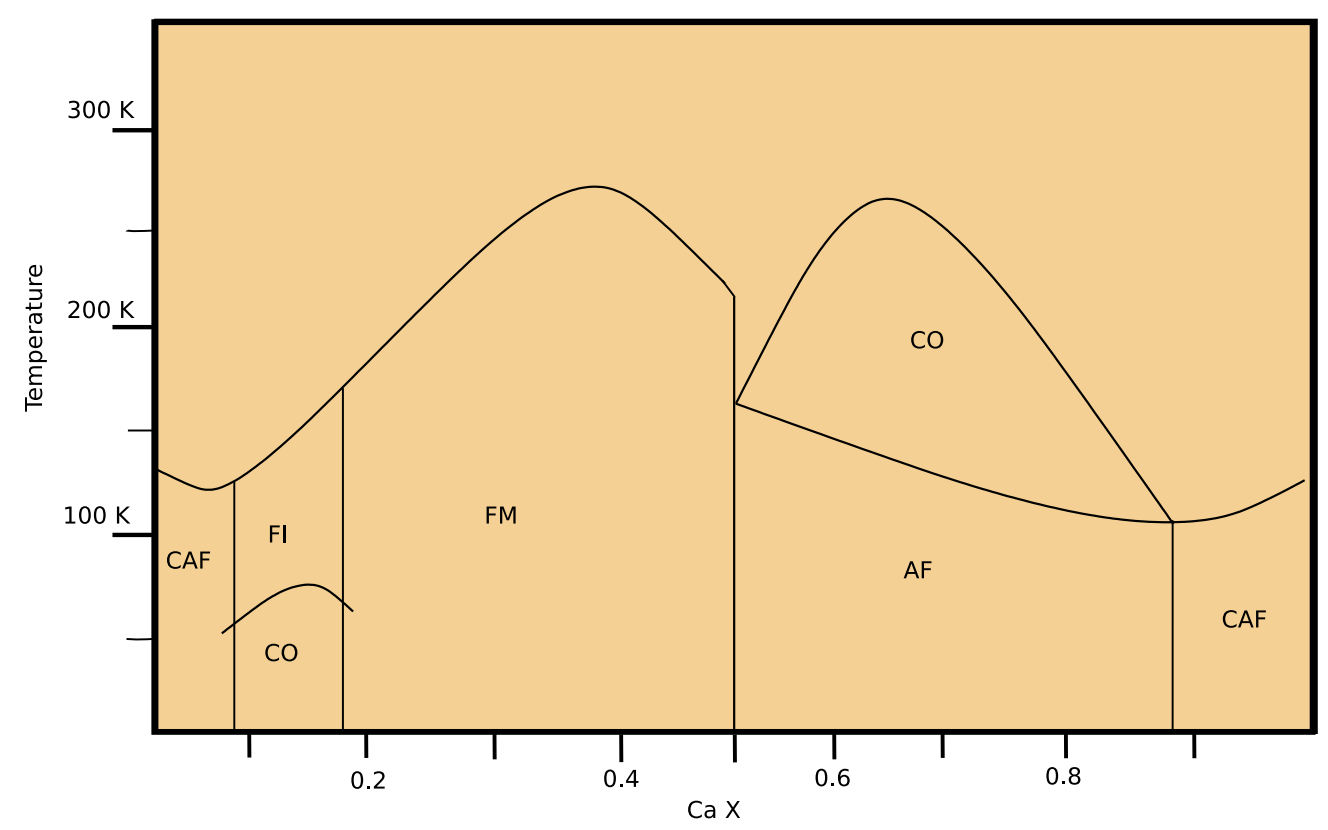

Figure 1.6: Magnetic Phase diagram of $\mathrm{La}_{1-x} \mathrm{Ca}_{x} \mathrm{MnO}_{3}$. Redrawn after S. Cheong and H. Hwang "Ferromagnetism vs. Charge/Orbital Ordering" in Colossal magneto resistive oxides by Y. Tokura [29].

leading to strong Coulomb interaction in the $e_{g}$-band. For understanding the physics of manganites, the Jahn-Teller distortion is crucial $[35,36]$. The symmetry reduction leads to the situation that the $e_{g}$-band is further split. This distortion especially occurs, if there is one $e_{g}$-electron at one site, corresponding to quarter-filling. The low temperature magnetic phase diagram is shown in figure 1.6 [29]. $\mathrm{LaMnO}_{3}$ is known to be a A-type antiferromagnet, meaning a ferromagnetic coupling in the orthorhombic planes and an antiferromagnetic coupling perpendicular. For small $x$ of Ca doping, the Jahn-Teller distortion is very strong, splitting the energy of the $e_{g}$-orbitals, and the system forms a canted antiferromagnetic state (CAF), which is orbitally ordered. For larger doping the system undergoes a transition to a ferromagnetic insulator (FI) and later for $0.2 \leq x \leq 0.5$ to a ferromagnetic metal (FM). This is the regime, where the CMR effect can be observed, as the paramagnetic phase above the Curie temperature is an insulator. For $x>0.5$ there are less than 0.5 electrons per site in the $e_{g}$-band, and the system forms an antiferromagnetic state again $(\mathrm{AF})$, where the electrons are localized in stripes. Such a charge ordering is denoted with $(\mathrm{CO})$ in the phase diagram. At the end of the $\mathrm{La}_{1-x} \mathrm{Ca}_{x} \mathrm{MnO}_{3}$ series is $\mathrm{CaMnO}_{3}$, which is like $\mathrm{LaMnO}_{3}$ an antiferromagnetic insulator (AF). This phase diagram obviously shows the competition between charge, spin and orbital order in the case of manganites. 


\subsection{Hubbard Model}

How should one describe such systems? A complete Hamiltonian would comprise the kinetic energy of each nuclei, the kinetic energy of each electron, the Coulomb interaction between the nuclei, the Coulomb interaction between the electrons and the nuclei and the Coulomb interaction between all electrons. As I want to describe macroscopic effects, in which at least $10^{23}$ particles are involved, the mentioned Hamiltonian is supposed to describe everything from plasma physics at high temperatures, every possible composition of the particles including solids and molecules, to low temperature collective phenomena like superconductivity and magnetism. As it is impossible and not intended to solve the emerging equations, the problem has to be drastically simplified: A solid consists of ions and electrons forming a three dimensional crystal. Since the ions are much heavier than the electrons, the velocity of the the nuclei is of the order $10^{-4}-10^{-5}$ smaller than the velocity of the electrons, which motivates to think of the ions as forming a rigid lattice. This ansatz is denoted as Born-Oppenheimer approximation [37]. With this approximation one completely rules out the possibility of melting and other lattice effects. Thus, structural changes as can be seen in manganites will not be taken into account in this work. Also phonons arising from lattice vibrations [37] are neglected in this thesis.

A model suitable for describing strongly interacting electrons on a lattice is the Hubbard model [38-40]. One way of motivating it, starts in the single electron picture trying to identify low temperature degrees of freedom and to extract interaction parameter ab initio. Therefore one attempts to solve for the oneelectron band-structure for the given periodic potential formed by the crystal structure. A method able to do so is the Density Functional Theory [41-43]. From the bands near the Fermi energy, which are the d-bands in transition metal oxides being most important for the low temperature physics, one can now construct Wannier functions. These states are well localized around each nucleus. With these states one can calculate the proper values for the electronelectron interaction [44-49] and set up a model Hamiltonian for the d-bands. I will now present another way for setting up a model Hamiltonian [50,51] for degenerate bands. This way is a bit less rigorous, but it helps understanding the underlying principles. The complete Hamiltonian is the sum of the single particle Hamiltonian $H_{T}$ and the interaction part $H_{U}$

$$
H=H_{T}+H_{U}
$$

One starts from the d-orbital wave functions of the transition metal $\Psi_{i, m, \sigma}(r)$, where $i$ labels the site, $m$ labels the orbital quantum number, $\sigma$ the spin quantum number, and $r$ corresponds to the position. The corresponding second quantized operator $c_{i, m, \sigma}^{\dagger}$ creates an electron with this wave function. One can 
now write the single particle Hamiltonian as

$$
\begin{aligned}
H_{T} & =\sum_{i j} \sum_{m} \sum_{\sigma} t_{i j, m} c_{i, m, \sigma}^{\dagger} c_{j, m, \sigma}, \\
t_{i j, m} & =\int d^{3} r \Psi_{i, m, \sigma}^{*}(r)\left(-\frac{\hbar^{2}}{2 m} \nabla^{2}+V(r)\right) \Psi_{j, m, \sigma}(r) .
\end{aligned}
$$

A problem with this approach is that it is difficult to determine the effective potential $V(r)$. If one assumes it to be the periodic potential of the nuclei, $t_{i j}$ will be negligible, because the transition metal atoms are far apart from each other. The exchange between different sites $i$ and $j$ is mediated by the oxygen p-orbitals. Thus, if one really wants to calculate the coupling between different sites, one has to include hopping processes mediated by the oxygen into the potential $V(r)$. The usual and simplest way is to guess some value for $t_{i j}$. Typical values are less than $1 \mathrm{eV}$ taking into account experiments on transition metal oxides.

The electron-electron interaction can be written as

$$
\begin{aligned}
H_{U} & =\sum_{i} \sum_{m_{1} \ldots m_{4}} \sum_{\sigma_{1} \ldots \sigma_{4}} U_{m_{1} \ldots m_{4}} c_{i, m_{1}, \sigma_{1}}^{\dagger} c_{i, m_{2}, \sigma_{2}}^{\dagger} c_{i, m_{3}, \sigma_{3}} c_{i, m_{4}, \sigma_{4}} \\
U_{m_{1} \ldots m_{4}} & =\int d^{3} r_{1} \int d^{3} r_{2} \Psi_{m_{1}}^{*}\left(r_{1}\right) \Psi_{m_{2}}^{*}\left(r_{2}\right) U\left(r_{1}-r_{2}\right) \Psi_{m_{3}}\left(r_{2}\right) \Psi_{m_{4}}\left(r_{1}\right) .
\end{aligned}
$$

Here as well, one cannot simply write $U\left(r_{1}-r_{2}\right) \sim \frac{e^{2}}{\left|r_{1}-r_{2}\right|}$ as the bare Coulomb interaction, as one would neglect the screening effects of the remaining s- and pelectrons. If one would calculate $U$ with the bare Coulomb interaction one finds values of about $U \approx 20 \mathrm{eV}$ [9], which are too large to describe real experiments. From experiments one can extract typical values of $U=4-8 \mathrm{eV}$ for transition metal oxides [9].

The two particle interaction can be assumed to be purely local, because the direct overlap between d-orbitals of different sites is negligible and screening effects reduce the interaction range. In equation (1.2) are several different matrix elements included. Due to the symmetry of the d-orbitals only even powers of the wave functions $\Psi_{m}$ lead to non-vanishing matrix elements [51]. The two particle interaction thus reads

$$
\begin{aligned}
H_{U}= & \sum_{i} \sum_{m} U_{m} n_{i, m, \uparrow} n_{i, m, \downarrow} \\
& +\sum_{i} \sum_{l<m, \sigma} U_{l m} n_{i, l, \sigma} n_{i, m,-\sigma} \\
& +\sum_{i} \sum_{l<m, \sigma}\left(U_{l m}-J_{l m}\right) n_{i, l, \sigma} n_{i, m, \sigma} \\
& -\sum_{i} \sum_{l<m, \sigma} J_{l m} c_{i, l, \sigma}^{\dagger} c_{i, l,-\sigma} c_{i, m,-\sigma}^{\dagger} c_{i, m, \sigma} \\
& +\frac{1}{2} \sum_{i} \sum_{l, m, \sigma} J_{l m} c_{i, l, \sigma}^{\dagger} c_{i, l,-\sigma}^{\dagger} c_{i, m,-\sigma} c_{i, m, \sigma}
\end{aligned}
$$




$$
\begin{aligned}
U_{m} & =\int d^{3} r_{1} \int d^{3} r_{2}\left|\Psi_{m}\left(r_{1}\right)\right|^{2} U\left(\left|r_{1}-r_{2}\right|\right)\left|\Psi_{m}\left(r_{2}\right)\right|^{2} \\
U_{l m} & =\int d^{3} r_{1} \int d^{3} r_{2}\left|\Psi_{l}\left(r_{1}\right)\right|^{2} U\left(\left|r_{1}-r_{2}\right|\right)\left|\Psi_{m}\left(r_{2}\right)\right|^{2} \\
J_{l m} & =\int d^{3} r_{1} \int d^{3} r_{2} \Psi_{l}^{*}\left(r_{1}\right) \Psi_{m}^{*}\left(r_{2}\right) U\left(\left|r_{1}-r_{2}\right|\right) \Psi_{l}\left(r_{2}\right) \Psi_{m}\left(r_{1}\right) .
\end{aligned}
$$

The operator $n=c^{\dagger} c$ is the density operator for an electron. Since the interaction is local and spin independent, the position index $i$ and the spin index $\sigma$ are neglected in the integrals. If one now assumes that the interaction parameters are the same for all d-orbitals, writing $U=U_{m}, U^{\prime}=U_{l m}$ and $J=J_{l m}$, one can further collect terms and ends up with

$$
\begin{aligned}
H_{U}= & U \sum_{i} \sum_{m} n_{i, m, \uparrow} n_{i, m, \downarrow}+\left(U^{\prime}-\frac{1}{2} J\right) \sum_{i} \sum_{l<m, \sigma, \sigma^{\prime}} n_{i, l, \sigma} n_{i, m, \sigma^{\prime}} \\
& -2 J \sum_{i} \sum_{l<m} \vec{S}_{i, l} \cdot \vec{S}_{i, m}+J \sum_{i} \sum_{l, m} c_{i, l, \uparrow}^{\dagger} c_{i, l, \downarrow}^{\dagger} c_{i, m, \downarrow} c_{i, m, \uparrow}
\end{aligned}
$$

The first two terms in equation (1.3) are intra-orbital and inter-orbital densitydensity interactions. The third term is a spin-spin interaction between different orbitals. It can be written as

$$
\vec{S}_{l} \cdot \vec{S}_{m}=\frac{1}{2}\left(S_{l}^{+} S_{m}^{-}+S_{l}^{-} S_{m}^{+}\right)+S_{l}^{z} S_{m}^{z}
$$

with spin-raising and -lowering operators $S^{+}$and $S^{-}$. The last term in equation (1.3) is a pair hopping term. In this work I will neglect this pair hopping term. This must be regarded as approximation to the real multi-orbital Hubbard model [52] and is done for numerical reasons. When neglecting this term, one can introduce an additional conserved orbital quantum number. As I will show later, this dramatically reduces the numerical effort. The spin-spin interaction, third term in equation (1.3), corresponds to the ferromagnetical Hund's coupling. Hund's rules state that all orbitals are first single occupied with electrons of parallel spin. This is represented by the spin-spin interaction, which lowers the energy if electrons occupy different orbitals with parallel spin. Of course, occupying different orbitals also avoids the energetic costs of the densitydensity interactions. The Hund's rule was not introduced artificially into this Hamiltonian but arises quite naturally from the general form of the two particle interaction. If all five d-bands are equivalent, the following equation for the interaction parameters holds [51]:

$$
U=U^{\prime}+2 J
$$

The multi-orbital Hubbard model $[50,51]$ is now the sum of the non-local single particle term (1.1) and the local interaction term (1.3)

$$
H=H_{T}+H_{U} \text {. }
$$

In some cases there is effectively only one d-band left at the Fermi energy. In this situation one can use the one-orbital Hubbard model [38-40], defined as

$$
H=\sum_{i j, \sigma} t_{i j} c_{i, \sigma}^{\dagger} c_{j, \sigma}+U \sum_{i} n_{i, \uparrow} n_{i, \downarrow}
$$


The interaction term now consists only of the intra-band interaction $U$. Made up of only two terms the model looks very simple. But the appearance is deceiving. There are only very few rigorous results [53-55]. The Hubbard model exhibits various phenomena like the metal insulator transition, antiferromagnetism, ferromagnetism and superconductivity, stating only some.

The model is easy to handle in the extreme limits of vanishing hopping, $t=0$, or vanishing interaction $U=0$. For vanishing interaction, $U=0$, one reproduces the energy bands for the d-orbitals from which the Hubbard model can be derived. The precise band structure depends on the lattice. The physics is determined by non-interacting electrons which fill the bands up to the Fermi energy. For a half-filled band the system shows metallic behavior. The other extreme case is a vanishing hopping amplitude $t$, but a finite and repulsive electron-electron interaction $U>0$. There is no coupling between the atoms. The ground state is a distribution of the electrons onto the atoms, trying to avoid double occupancies, if possible, and is therefore highly degenerate. This ground state is clearly insulating. The competition between both terms in equation (1.5), the tendency of delocalization versus localization, leads to the interesting phenomena just mentioned above for different materials.

In this thesis I will focus on the one-orbital Hubbard model (1.5) and the twoorbital Hubbard model, in which the orbital index in equation (1.3) can take only the values $m=\{1,2\}$. The two-orbital Hubbard model is a very good model for describing the physics of a correlated $e_{g}$-band. It takes into account the strong local electron-electron interactions, the hopping from one atom to another, and the orbital degeneracy. More precisely, it represents the situation of transition metal oxides with octahedral symmetry, where the Fermi energy lies within the $e_{g}$-band. The model completely neglects the $t_{2 g}$-band. The coupling of the $t_{2 g}$-band to the $e_{g}$-band due to Hund's coupling can be very strong as in the case of manganites [33]. Furthermore, the model does not include any coupling between the electrons and the lattice. The lattice, given by the hopping parameter $t_{i j}$, is fixed and there is no coupling to phonons created by lattice vibrations. So one cannot expect to see all the aspects of transition metal oxides. Nevertheless, as I will show in this work, the twoorbital Hubbard model shows a very interesting ground state phase diagram with different ordered phases. It is the basic model for investigating the physics of strongly correlated electron systems including orbital degeneracy $[50,51]$.

\subsection{Exchange Interactions}

After introducing the model and its parameters I will shortly discuss two very important exchange mechanisms between different transition metal atoms. The exchange is mediated by the oxygen p-orbitals. Oxygen has the strong tendency to fill its p-orbitals to establish a completely filled shell like Neon [10]. Thus, one can assume that after an electron has moved from an oxygen atom to a transition metal atom, immediately another electron will fill up this p-shell of the oxygen. This results in a rather weak hopping amplitude $t$ between the transition metal d-orbitals. The resulting coupling between the two transition 
metal atoms is called indirect exchange. One remarkable point is, that although there is no magnetic interaction between both transition metal atoms, there is a tendency for aligning the spins of the electrons either parallel or antiparallel depending on the precise situation. As I will show now, this alignment is only due to the Coulomb interaction and the Pauli exclusion principle. The most important processes in transition metal oxides are called super exchange and double exchange.

\section{Super Exchange}

I will firstly address the super exchange $[56,57]$. It is called like this, because it extends the normally short range direct exchange to a longer range. For a better understanding, I will look at the relevant states of two transition metal atoms. The situation is such that there is only one possible state at each of the two atoms. Altogether there are two electrons in the system. Thus we can assume that the basis for this problem consists of 6 states, reading

$$
\begin{array}{rlrl}
|1\rangle & =|\uparrow\rangle_{1}|\uparrow\rangle_{2}, & |2\rangle=|\downarrow\rangle_{1}|\downarrow\rangle_{2}, & |3\rangle=|\uparrow\rangle_{1}|\downarrow\rangle_{2}, \\
|4\rangle=|\downarrow\rangle_{1}|\uparrow\rangle_{2}, & |5\rangle=|\uparrow \downarrow\rangle_{1}|0\rangle_{2}, & |6\rangle=|0\rangle_{1}|\uparrow \downarrow\rangle_{2},
\end{array}
$$

where the index at each "ket" represents the index of the atom and the arrow denotes the alignment of the electron. The case of an empty atom is denoted as $|0\rangle$. I assume that there is a strong but finite repulsive interaction with amplitude $U$, if two electrons are situated at the same atom. This is the case for the states $|5\rangle$ and $|6\rangle$. Secondly, there is a possible hopping of an electron from one atom to the other with amplitude $-t$. There are no spin flip interactions. Thus the Hamiltonian matrix can be written as

$$
H=\left(\begin{array}{cccccc}
0 & 0 & 0 & 0 & 0 & 0 \\
0 & 0 & 0 & 0 & 0 & 0 \\
0 & 0 & 0 & 0 & -t & -t \\
0 & 0 & 0 & 0 & -t & -t \\
0 & 0 & -t & -t & U & 0 \\
0 & 0 & -t & -t & 0 & U
\end{array}\right) .
$$

While the parallel configurations, states $|1\rangle$ and $|2\rangle$, have ground state energy $E=0$, there is one mixed state, mainly consisting of the states with spin up at one atom and spin down at the other atom, which has energy $E=\frac{1}{2} U-\frac{1}{2} \sqrt{U^{2}+16 t^{2}} \approx-8 \frac{t^{2}}{U}$ for large $U$. This energy gain of an antiparallel spin state is called super exchange, and can lead to an antiferromagnetic ground state in an extended system, where all nearest neighbor electrons are aligned antiparallel. A well known example for a transition metal oxide with antiferromagnetic structure due to super exchange is $\mathrm{NiO}$ [1].

\section{Double Exchange}

Indirect exchange can also lead to a ferromagnetic coupling. A first clear sign was found in 1950 in the manganite compound $\mathrm{La}_{1-x} \mathrm{Ca}_{x} \mathrm{MnO}_{3}$ [58]. The results could be explained by the indirect exchange called double exchange [59-61]. The 


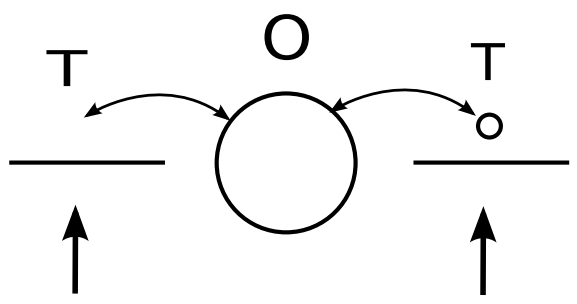

Figure 1.7: Typical situation of a double exchange process. For each transition metal atom " $\mathrm{T}$ " there is one localized magnetic moment and one itinerant level. Oxygen "O" acts as intermediary for the hopping.

situation is such that there is a localized moment at each transition metal atom and one electron, which can move from one atom to another by hybridization through the oxygen atom, see figure 1.7. One can imagine that the localized moments are formed by a half-filled $t_{2 g}$-shell and the itinerant electron occupies an $e_{g}$-state. An important point is that there is only one itinerant electron for two transition metal atoms, which couples locally via Hund's coupling to the local moments. As there are no spin flip processes, I assume that the single electron is an up-electron. A basis for this problem is given by

$$
\begin{array}{rlrl}
|1\rangle & =|\Uparrow, \uparrow\rangle_{1}|\Uparrow, 0\rangle_{2}, & |2\rangle=|\Uparrow, 0\rangle_{1}|\Uparrow, \uparrow\rangle_{2}, & |3\rangle=|\Downarrow, \uparrow\rangle_{1}|\Uparrow, 0\rangle_{2}, \\
|4\rangle=|\Downarrow, 0\rangle_{1}|\Uparrow, \uparrow\rangle_{2}, & |5\rangle=|\Uparrow, \uparrow\rangle_{1}|\Downarrow, 0\rangle_{2}, & |6\rangle=|\Uparrow, 0\rangle_{1}|\Downarrow, \uparrow\rangle_{2}, \\
|7\rangle=|\Downarrow, \uparrow\rangle_{1}|\Downarrow, 0\rangle_{2}, & |8\rangle=|\Downarrow, 0\rangle_{1}|\Downarrow, \uparrow\rangle_{2}, &
\end{array}
$$

where the double arrow represents the localized moment and the small arrow the electron which is either at atom 1 or atom 2. The Hamilton matrix for this problem reads,

$$
H=\left(\begin{array}{cccccccc}
-J & -t & 0 & 0 & 0 & 0 & 0 & 0 \\
-t & -J & 0 & 0 & 0 & 0 & 0 & 0 \\
0 & 0 & J & -t & 0 & 0 & 0 & 0 \\
0 & 0 & -t & -J & 0 & 0 & 0 & 0 \\
0 & 0 & 0 & 0 & -J & -t & 0 & 0 \\
0 & 0 & 0 & 0 & -t & J & 0 & 0 \\
0 & 0 & 0 & 0 & 0 & 0 & J & -t \\
0 & 0 & 0 & 0 & 0 & 0 & -t & J
\end{array}\right) .
$$

The ground state of this system is a combination of the first two states with energy $E=-J-t$, where $J>0$ is the Hund's coupling and $t$ the hopping amplitude. This state is clearly a "ferromagnetic" state, as both moments and the electron point into the same direction.

\subsection{Types of Magnetic Order}

These very simplified models already show that depending on the exact situation magnetic moments may align parallel or antiparallel. In general, for 

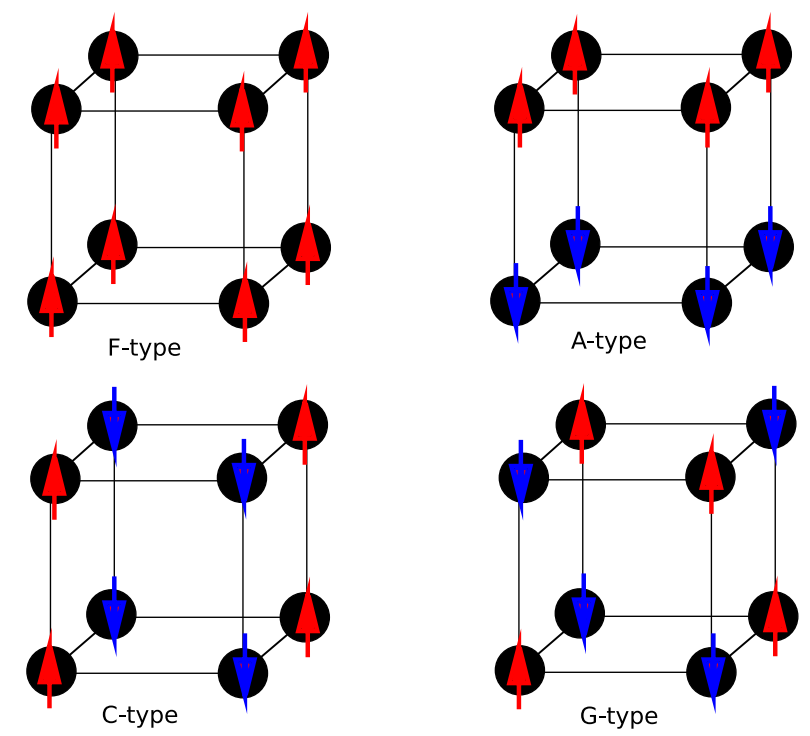

Figure 1.8: Different possible magnetic orders for a simple cubic lattice. Red arrows correspond to up electrons and blue arrows to down electrons. See text for explanation.

real materials more complex arrangements are possible. The phase diagram of $\mathrm{La}_{1-x} \mathrm{Ca}_{x} \mathrm{MnO}_{3}$, chapter 1.2.4, is a characteristic example. Figure 1.8 shows some common spin arrangements for a cubic lattice [29]. The F-type denotes an ordinary ferromagnetic state, while in the A-type electrons in one layer are ordered ferromagnetically but the layers itself are ordered antiferromagnetically. In the C-type the moments lying along the z-direction are aligned ferromagnetically. The G-type order represents a Néel-state in which all nearest neighbors are aligned antiparallel. Nevertheless, these four types are only very simple examples. In nature the situation if often more complex involving charge, spin and orbital degrees of freedom. In figure 1.8 only situations were shown where the moments are parallel or antiparallel. But the direction of the moments may change by a smaller angle than $180^{\circ}$ leading to a canted state or an order with a periodicity larger than two. In the latter case one speaks of spin density waves.

\subsection{Outline}

The further outline of this work is as follows. In the next two chapters I will explain the theoretical foundations of the later calculations. I will introduce the dynamical mean field theory and the Bethe lattice, for which I performed the calculations. As the dynamical mean field theory relates the lattice problem onto a quantum impurity model, I will present in chapter 3 the numerical renormalization group and the density matrix renormalization group, which both can be used for solving impurity models.

After introducing their theoretical concepts in chapter 3, chapter 4 directly addresses a comparison of both impurity solvers for some selected situations, which must be handled when trying to calculate magnetic phases within the 
dynamical mean field theory.

Finally, chapter 5 and 6 are dedicated to the results for the magnetic phases of the Hubbard model. Chapter 5 deals with the one-orbital situation. The possible magnetic states are analyzed over a wide range of the interaction parameters also varying the form of the electron hopping $t_{i j}$. The form of the hopping parameter is changed by introducing a next nearest neighbor hopping term, which has influence on the metal insulator transition, the antiferromagnetic Néel-state, and the possible ferromagnetic states. Chapter 6 analyzes the magnetic properties of the two-orbital Hubbard model. There one can observe the competition of the just mentioned super exchange and the double exchange for fillings of $\langle n\rangle \approx 1.5$. Besides this, quarter filling represents a situation, where one can observe a clash of several different phases corresponding to the spin, charge, and orbital degrees of freedom. Finally, I will summarize this work and give a short outlook. 
CHAPTER 2

\section{Dynamical Mean Field Theory}

\subsection{Introduction}

This chapter gives an introduction to the Dynamical Mean Field Theory, which I have used for solving the lattice model. I will discuss the mathematical derivation of the method and the approximations made. As the main goal of this work is the calculation of magnetic phase diagrams of the Hubbard model and properties of the system within these phases, I will show how to stabilize magnetic ordered solutions. In the last section of this chapter, I will introduce the lattice for which the calculations were performed.

\subsection{Cavity Construction}

For the sake of simplicity of the derivation, I will use a simple example. So let me first introduce the Ising model $[62,63]$. Let there be a number of independent variables $\sigma_{i}=\{-1,1\}$. One can assume these variables to be spins on a lattice. The Hamiltonian for this model reads

$$
H=\frac{1}{2} \sum_{i, j} J_{i, j} \sigma_{i} \sigma_{j}-h \sum_{i} \sigma_{i} .
$$

The constants $J_{i, j}$ represent a coupling between the spins, and $h$ is a homogeneous magnetic field acting on all spins. The property, which completely describes the physics and behavior of the system in equilibrium for a given temperature $T$, is the free energy $F$ or the partition function $Z$, respectively,

$$
\begin{aligned}
Z\left(\beta, J_{i, j}, h\right) & =\underset{\{\sigma\}}{\mathcal{T}_{r}} \exp (-\beta H) \\
F\left(\beta, J_{i, j}, h\right) & =-\frac{1}{\beta} \log Z,
\end{aligned}
$$


where $\beta=1 /\left(k_{B} T\right)$. Expectation values and correlation functions can be easily calculated by derivatives of the free energy. For example the magnetization, given by $M=\sum_{i} \mathcal{T}_{\sigma}\left(\sigma_{i} \exp (-\beta H)\right) / Z$, can be written as

$$
M=-\frac{d F\left(\beta, J_{i, j}, h\right)}{d h} .
$$

The magnetization represents an order parameter for the ferromagnetic phase transition occurring in this model $[62,63]$. The Ising model at $T=0$ with ferromagnetic coupling $J_{i, j}<0$ between nearest neighbors only is clearly ferromagnetic. The lowest energy configuration is such, that all spins are aligned in the same direction. At this point the system is $Z_{2}$ symmetric, meaning that the ground state is twofold degenerate. The value of all spins is simultaneously either $\sigma=1$ or $\sigma=-1$. At very high temperatures, $J_{i, j} \ll k_{B} T$, the system will be in the paramagnetic state, where all spins are in principle decoupled and thus $M=0$. The existence of a finite temperature, $T>0$, below which the spins begin to order, critically depends on the dimension of the system. For dimension $d=1$, the spins align only for $T=0[62,63]$, while for $d=2$ the system exhibits a finite temperature phase transition, as found by Onsager [64]. Already for the three dimensional system there is no analytical solution to this problem.

For strongly interacting electrons on a lattice, as introduced in chapter 1.3, the situation is even more difficult. Nevertheless, there are numerical and analytical methods to study strongly correlated electron systems. A numerical method that is able to directly simulate a lattice model is Quantum Monte Carlo [6567]. But Quantum Monte Carlo is limited to small systems and simulations of rather high temperatures due to the computational effort. Besides this, there are parameter regions where the simulations will fail because of the signproblem $[68,69]$. There are also other approaches like exact diagonalization, which is limited to even smaller clusters. These examples are by no means all in the zoo of possible methods for such systems. I wanted to illustrate that there are different methods all having advantages and disadvantages. There is no best method for the model I am intending to study. An overview about analytical and numerical methods for interacting quantum systems focusing on metal insulator transitions can be found in M. Imada [14].

In this work I use the dynamical mean field theory. Using this approach it is possible to scan through the whole parameter region, temperature and interaction parameters. I am able to identify and analyze different phases. Of course, this method has also some drawbacks. Besides the approximations, which I will state below, there are also sometimes problems stabilizing ordered phases. I will come back to this point later.

The aim of any mean field theory is to relate the lattice problem to a pure local problem, called impurity problem, by partially tracing out all degrees of freedom but one site, here denoted as "site 0". This is called a cavity construction [70]. For this purpose, one splits the trace of the partition function into a part containing only degrees of freedom of "site 0 ", $\mathcal{T} r_{0}$, and one part containing the rest of the system, $\mathcal{T}_{r}$. The Hamiltonian is split into three parts: $H_{0}$ containing only parts of the single site $0, H_{0}^{C}$ parts which connect the single 
site to the rest of the system and $H^{C}$ the rest of the system, which contains no degrees of freedom from the single site. The partition function for the Ising model can then be written as

$$
\begin{aligned}
Z & =\mathcal{T r} \exp (-\beta H) \\
& =\mathcal{T}_{\{0\}}\left[\exp \left(-\beta H_{0}\right){ }_{\{C\}}^{\mathcal{T}_{r}}\left[\exp \left(-\beta H_{0}^{C}\right) \exp \left(-\beta H^{C}\right)\right]\right]
\end{aligned}
$$

since it denotes a classical model. Expanding $\exp \left(-\beta H_{0}^{C}\right)$, one finds

$$
\begin{aligned}
Z= & \mathcal{T r}_{r} \exp (-\beta H) \\
= & \mathcal{T}_{\{0\}}\left[\operatorname { e x p } ( \beta h \sigma _ { 0 } ) \mathcal { T } _ { \{ C \} } \left[\exp \left(-\beta \frac{1}{2} \sigma_{0} \sum_{i \neq 0}\left(J_{0 i}+J_{i 0}\right) \sigma_{i}\right)\right.\right. \\
& \left.\left.\exp \left(-\beta\left(\frac{1}{2} \sum_{i, j \neq 0} J_{i j} \sigma_{i} \sigma_{j}-h \sum_{i \neq 0} \sigma_{i}\right)\right)\right]\right] \\
= & \mathcal{T}_{\{0\}}\left[\operatorname { e x p } ( \beta h \sigma _ { 0 } ) \underset { \{ C \} } { \mathcal { T } _ { r } } \left[\sum_{n=0}^{\infty} \frac{1}{n !}\left(-\beta \frac{1}{2} \sigma_{0} \sum_{i \neq 0}\left(J_{0 i}+J_{i 0}\right) \sigma_{i}\right)^{n}\right.\right. \\
& \left.\left.\exp \left(-\beta\left(\frac{1}{2} \sum_{i, j \neq 0} J_{i j} \sigma_{i} \sigma_{j}-h \sum_{i \neq 0} \sigma_{i}\right)\right)\right]\right]
\end{aligned}
$$

If one now performs the trace over the system without site 0 , one can perform a cumulant expansion and obtains

$$
\begin{aligned}
Z= & \underset{\{0\}}{\mathcal{T}_{r}}\left[\operatorname { e x p } \left(\beta h \sigma_{0}+\sum_{n=1}^{\infty} \frac{\left(\sigma_{0}\right)^{n}}{n !}\right.\right. \\
& \left.\left.\sum_{i_{1} \ldots i_{n}}(-\beta)^{n}\left(\prod_{k=1}^{n} \frac{1}{2}\left(J_{0 i_{k}}+J_{i_{k} 0}\right)\right)\left\langle\sigma_{i_{1}} \ldots \sigma_{i_{n}}\right\rangle_{\text {cum }}^{C}\right)\right]
\end{aligned}
$$

Here $\langle\cdot\rangle_{\text {cum }}^{C}$ denotes the cumulant within the cavity system. Until now everything is exact. But nothing was gained, as the new action contains all powers of $\sigma_{0}$ and infinite many cumulant expectation values have to be calculated.

The first approximation is to assume that the expectation value in the system without site 0 is the same as in the system with site 0 , so $\langle\cdot\rangle_{\text {cum }}^{C}=\langle\cdot\rangle_{\text {cum }}$. The next step is to neglect all terms in the sum for $n>1$. Both approximations can be justified by an infinite coordination number $z$ and an infinite extended lattice. One ends up with the following formula

$$
Z=\underset{\left\{\sigma_{0}\right\}}{\mathcal{T} r} \exp \left(-\beta \sigma_{0}\left(-h+\frac{1}{2} \sum_{i}\left(J_{0 i}+J_{i 0}\right)\left\langle\sigma_{i}\right\rangle\right)\right)
$$

where $\left\langle\sigma_{i}\right\rangle_{\text {cum }}=\left\langle\sigma_{i}\right\rangle$ holds. Analyzing a homogeneous system with interactions between $z$ nearest neighbors only

$$
J_{0 i}=J_{i 0}=\left\{\begin{array}{ll}
J & \text { i is nearest neighbor of } 0 \\
0 & \text { else }
\end{array},\right.
$$


the partition function for the Ising model in the mean field approximation reads

$$
Z=\mathcal{T} r[\exp (-\beta \sigma(-h+z J\langle\sigma\rangle))]
$$

where $\langle\sigma\rangle$ has to be calculated self-consistently. It can be shown that this formula is exact in the limit of an infinite dimensional lattice, in the sense of an infinite coordination number $z$. There one has to scale $z J \rightarrow J^{\star}=$ const for $z \rightarrow \infty$ [70]. Calculating the expectation value $\langle\sigma\rangle$ finally yields the familiar selfconsistency equation in the Weiss molecular field theory of the ferromagnet [71]

$$
m=\tanh \left(-\beta\left(-h+J^{\star} m\right)\right) .
$$

A spontaneous magnetization, $h=0$ but $m \neq 0$, can occur for $\beta\left|J^{\star}\right|>1$ leading to a finite temperature phase transition at $T=\left|J^{\star}\right| / k_{B}$.

\subsection{Dynamical Mean Field Theory}

The next step is to formulate this theory for a quantum mechanical system like the Hubbard model [70,72-80]. I will present a derivation for the one-orbital Hubbard model, as the calculations do not change for a multi-orbital Hubbard model. The main difference between both Hamiltonian is the local interaction part, which is absorbed unchanged into the effective action derived. The partition function must now be written as a functional integral over Grassmann variables [81]

$$
\begin{aligned}
Z= & \int \prod_{i} D c_{i, \sigma}^{\dagger} D c_{i, \sigma} \exp (-S) \\
S= & \int_{0}^{\beta} d \tau\left(\sum_{i, \sigma} c_{i, \sigma}^{\dagger}(\tau) \partial_{\tau} c_{i, \sigma}(\tau)+\sum_{i j, \sigma} t_{i j} c_{i, \sigma}^{\dagger}(\tau) c_{j, \sigma}(\tau)\right. \\
& \left.-\mu \sum_{i, \sigma} n_{i, \sigma}(\tau)+U \sum_{i} n_{i, \uparrow}(\tau) n_{i, \downarrow}(\tau)\right) .
\end{aligned}
$$

The imaginary time argument $\tau$ of the Grassmann variables will be dropped in the following formulas. With respect to a single site 0 , one again splits up the action into the part of the single site $S_{0}$, the part connecting the single site with the lattice $S_{0}^{C}$, and the rest of the action $S^{C}$. Expanding the action in the $S_{0}^{C}$ part, one again obtains an effective action for the single site [70]

$$
\begin{aligned}
S_{\mathrm{eff}}= & \int_{0}^{\beta} d \tau\left(\sum_{\sigma} c_{0, \sigma}^{\dagger}\left(\partial_{\tau}-\mu\right) c_{0, \sigma}+U n_{0, \uparrow} n_{0, \downarrow}\right) \\
& +\sum_{\sigma} \sum_{n=1}^{\infty} \frac{1}{n !} \sum_{i_{1} \ldots j_{n}} \int_{0}^{\beta} d \tau_{i_{1}} \ldots d \tau_{j_{n}}\left(t_{0 i_{1}} c_{0, \sigma}^{\dagger} \ldots t_{0 i_{n}} c_{0, \sigma}^{\dagger}\right. \\
& \left.G_{i_{1} \ldots j_{n}}^{C, \text { con }}\left(\tau_{i_{1}} \ldots \tau_{j_{n}}\right) t_{j_{1} 0} c_{0, \sigma} \ldots t_{j_{n} 0} c_{0, \sigma}\right) .
\end{aligned}
$$


Instead of the expectation values $\left\langle\sigma_{i_{1}} \ldots \sigma_{i_{n}}\right\rangle$ in the Ising model, the effective field is now given by the connected Green's functions of the cavity system $G^{C, c o n}$. One can now perform the same approximations as above and neglect all terms with $n \geq 2$ in this action. This results in an effective field, which only contains the one-particle Green's function, see textbook definition [82],

$$
\begin{aligned}
G^{C}\left(\tau-\tau^{\prime}\right) & =-\left\langle\mathcal{T} c(\tau) c^{\dagger}\left(\tau^{\prime}\right)\right\rangle_{C} \\
G^{C}\left(i \omega_{n}\right) & =\int_{0}^{\beta} \exp \left(i \omega_{n} \tau\right) G^{C}(\tau) \\
G^{C}(\zeta) & =\frac{1}{\zeta-\Sigma(\zeta)}
\end{aligned}
$$

in the action of the cavity Hamiltonian $\langle\cdot\rangle_{C}$. The third line in equation (2.1) follows from analytic continuation of the the second line introducing the selfenergy of the system $\Sigma(\zeta)$ [81]. I can neglect the subscript "con", as the usual one-particle Green's function equals the definition of the connected one-particle Green's function. The effective action is now given by

$$
\begin{aligned}
S_{\mathrm{eff}}= & \int_{0}^{\beta} d \tau d \tau^{\prime} \sum_{\sigma} c_{0, \sigma}^{\dagger}(\tau)\left(\left(\partial_{\tau}-\mu\right) \delta\left(\tau-\tau^{\prime}\right)+\sum_{i j \neq 0} t_{i 0} t_{j 0} G_{i j}^{C}\left(\tau-\tau^{\prime}\right)\right) c_{0, \sigma}\left(\tau^{\prime}\right) \\
& +\int_{0}^{\beta} d \tau U n_{0, \uparrow}(\tau) n_{0, \downarrow}(\tau) .
\end{aligned}
$$

The effective field $G_{i j}^{C}(\tau)$ depends on the imaginary time $\tau$, hence the name dynamical mean field theory. Although this problem contains only degrees of freedom of a single site, there is no explicit solution for arbitrary $G_{i j}^{C}$. This effective single site problem corresponds to a quantum impurity problem. The relation between this effective action and that of a quantum impurity model is shown in the next chapter. There I will also discuss two numerical approaches to obtain the one-particle Green's function for such models.

At this point I will bring this effective action into a more appropriate form introducing the effective field $\mathcal{G}$ :

$$
S_{\mathrm{eff}}=:-\int_{0}^{\beta} d \tau d \tau^{\prime} \sum_{\sigma} c_{0, \sigma}^{\dagger} \mathcal{G}^{-1}\left(\tau-\tau^{\prime}\right) c_{0, \sigma}+U n_{0, \uparrow} n_{0 \downarrow} .
$$

Fourier transformation and analytic continuation with respect to $\tau$ of the noninteracting part of this action yields

$$
\mathcal{G}^{-1}(\omega+i 0):=\omega+i 0+\mu-\sum_{i j} t_{0 i} t_{0 j} G_{i j}^{C}(\omega+i 0)
$$

The shape of the lattice only enters in the sum over all Green's functions $\sum_{i j} t_{0 i} t_{0 j} G_{i j}^{C}(\omega+i 0)$. Doing the Fourier transformation in the lattice sites [70], one ends up with

$$
\mathcal{G}^{-1}(\omega+i 0)=\Sigma(\omega+i 0)+\left(\int d \epsilon \frac{\rho(\epsilon)}{\omega+i 0+\mu-\Sigma(\omega+i 0)-\epsilon}\right)^{-1}
$$


where $\rho(\epsilon)$ is the density of states of the non-interacting lattice problem. One uses here that the self-energy is purely local $\Sigma_{i j}=\Sigma \delta_{i j}[75-77]$. The complete lattice structure and all hopping terms are included in $\rho(\epsilon)$. This integral is the only point in the DMFT, where the lattice structure enters.

As one cannot calculate the Green's function (2.1) explicitly for an arbitrary impurity action, I will solve the self-consistency equation $(2.1,2.2)$ by an iterative procedure. Starting with an arbitrary self-energy, which can be zero, one calculates the effective action and solves the corresponding impurity problem for a new self-energy. This must be repeated until the self-energy does not change anymore. This procedure is shown in figure 2.1.

Summarized, one has done the following approximations: One neglects all terms of higher order than one in the expansion of the effective action, and one assumes the self-energy to be local. One should ask, how good are these approximations? Are they controlled and is there a limit, where these equations are exact? The answer to the last question is affirmative. In the limit of infinite dimensions or infinite coordination number, the dynamical mean field theory becomes exact. The crucial point in this limit is that one has to scale the hopping parameter $t_{i j}$ as

$$
t_{i j} \sqrt{z}^{|i-j|} \longrightarrow t_{i j}^{\star}
$$

to get a non-trivial solution for the physical properties of the system [75]. Taking the limit $z \longrightarrow \infty$, one can proof that all non-local contributions to the selfenergy vanish and that the truncation of the terms in the effective action is exact [75].

The DMFT must be considered as an approximation for real materials. Nevertheless, calculations showed that within the DMFT a large amount of physical properties of, for example, transition metal compounds can be analyzed, and also compared to experiments at least qualitatively, sometimes even quantitatively [83-87]. The actual structure of the lattice does appear in the DMFT equation only through the local density of states. It has a profound effect on the details of the resulting physics. In particular, different lattice types are still discriminated by the actual form of the DOS and asymmetries give rise to competitions between interactions. Therefore the DMFT is a good starting point to analyze and understand the very difficult many body effects seen in real materials.

\subsection{Magnetic solutions}

\subsubsection{Ferromagnetism}

In this work I will concentrate on magnetically ordered states. With the DMFT I am able to solve the lattice problem and obtain the lattice Green's function or self-energy. In the above equations the effective field $\mathcal{G}(2.2)$ was completely spin independent. Due to the SU2 symmetry of the Hamiltonian, one cannot expect to obtain a magnetically ordered state as solution. To achieve this, one has to calculate independent effective fields for the spin-up and spin-down 


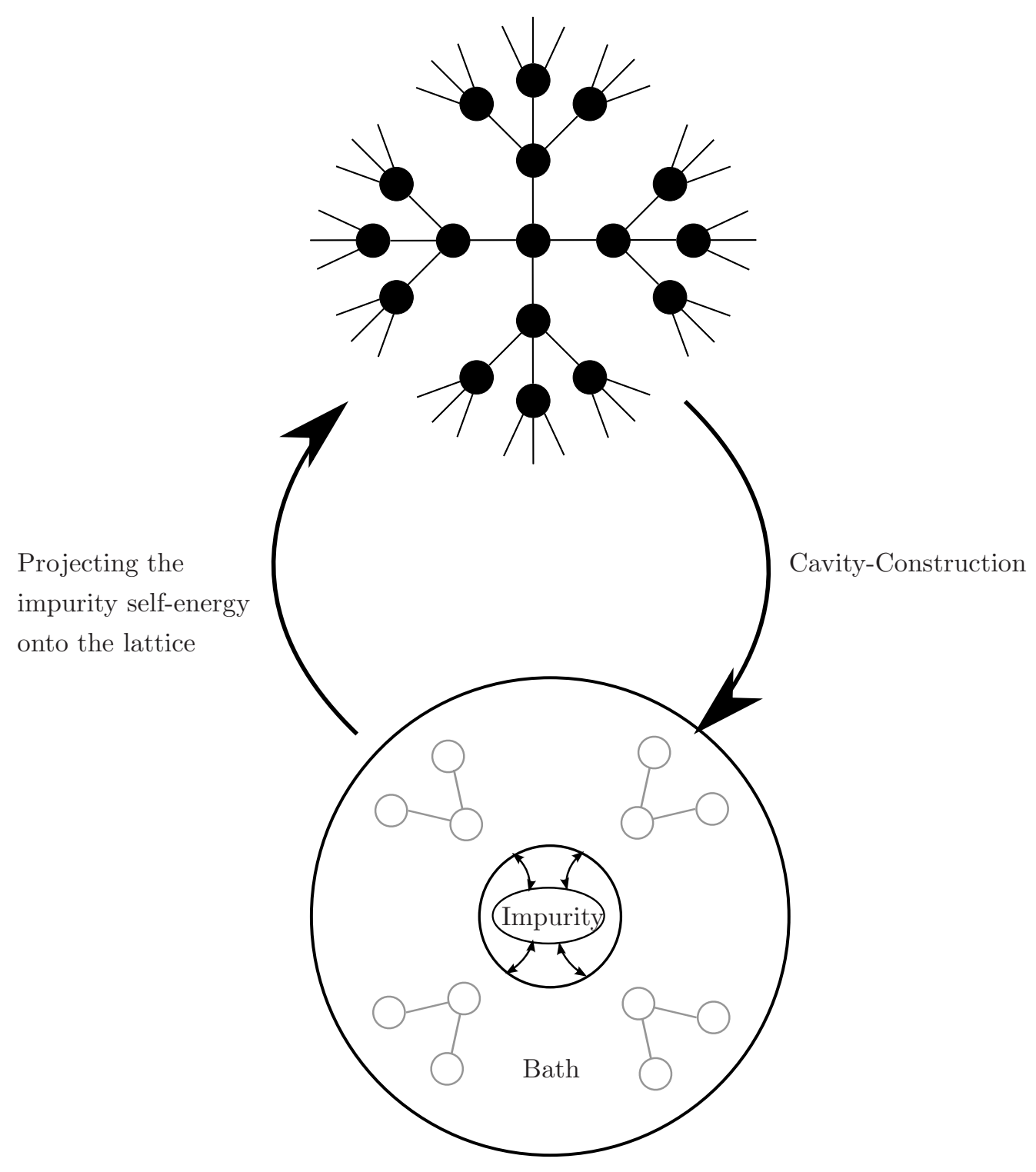

Figure 2.1: Iterative DMFT-loop. The lattice is projected onto an impurity problem. The solution of the impurity calculation is projected back onto the lattice. This is repeated until convergence. 
components $[70]$

$$
\mathcal{G}_{\sigma}^{-1}\left(i \omega_{n}\right)=\Sigma_{\sigma}\left(i \omega_{n}\right)+\left(\int d \epsilon \frac{\rho(\epsilon)}{i \omega_{n}+\mu-\Sigma_{\sigma}\left(i \omega_{n}\right)-\epsilon}\right)^{-1} .
$$

Additionally, it is important to break the SU2 symmetry of the system in the very first iteration by applying a small magnetic field [70]. After the first iteration I switch off the magnetic field and continue as described in figure 2.1. If the system possesses a ferromagnetic instability, it will evolve into a selfconsistent solution with $\Sigma_{\uparrow} \neq \Sigma_{\downarrow}$ and a polarization $p=\frac{\left|n_{\uparrow}-n_{\downarrow}\right|}{n_{\uparrow}+n_{\downarrow}}>0$. The occupations can be calculated from the self-energy by

$$
n_{\sigma}=-\frac{1}{\pi} \int d \omega f(\omega) \mathfrak{I m} \int d \epsilon \frac{\rho(\epsilon)}{\omega+i 0+\mu-\Sigma_{\sigma}(\omega)-\epsilon},
$$

where $f(\omega)$ denotes the Fermi-function.

\subsubsection{Antiferromagnetism}

I will also present solutions with antiferromagnetic long-range order. The important point is, that this ordered state is non-homogeneous; neighboring sites, A and B, have different self-energies [70,73]. Focusing on the Néel-state, the following relation holds

$$
\Sigma_{A, \sigma}=\Sigma_{B,-\sigma}
$$

To derive the proper equation for the effective field, one has to assume that the lattice is bipartite. This means that one can divide the lattice into two sublattices, and each site of the A-sublattice has only B-sites as nearest neighbors. Then one can show that the self-consistency condition still holds, but now in matrix form of the two sublattices [70,73]. The Green's function entering must be calculated by matrix inversion

$$
\begin{aligned}
\mathbf{G}\left(\zeta_{A}, \zeta_{B}\right) & =\int d \epsilon \rho(\epsilon)\left(\left(\begin{array}{cc}
\zeta_{A \sigma} & 0 \\
0 & \zeta_{B \sigma}
\end{array}\right)-\mathbf{H}_{T}\right)^{-1} \\
& =\int d \epsilon \rho(\epsilon)\left(\begin{array}{cc}
\zeta_{A \sigma} & -\epsilon \\
-\epsilon & \zeta_{B \sigma}
\end{array}\right)^{-1} .
\end{aligned}
$$

The matrix $H_{T}$ represents the hopping term between the sublattices. Using equation (2.4) one can now calculate the new effective fields for both spin components and sublattices. As relation (2.3) holds for the two sublattices, one still needs to solve only one impurity problem per DMFT iteration, as interchanging spin labels yield the solution for the other sublattice.

\subsection{Bethe lattice}

In this last section of the chapter, I will introduce the Bethe lattice, for which I have performed the calculations. The lattice itself is very artificial. The construction of the Bethe lattice with coordination number $z$ is very easily done in shells: 
- Begin with drawing one site, which is shell 0.

- Connect the first site with $z$ neighboring sites, building shell 1 .

- Connect all sites of the last shell with $z-1$ new sites, building the new shell.

- There are no closed loops.

Because of this symmetric construction of the shells and the important point that there are no closed loops, implying that there is only one way from site A to site B, many models can be solved exactly for the Bethe lattice [88]. However, for the Hubbard model this is in general not the case, but one can solve the model without two particle interaction $U=0$ [70]. Moreover, one is able to calculate the density of states (DOS) of the lattice, which is essential for the DMFT. As the Bethe lattice is bipartite, I am able to perform antiferromagnetic calculations.

The DOS $\rho(\epsilon)$ is given by the imaginary part of the local Green's function as $\rho(\epsilon)=-1 / \pi \mathfrak{I m} G(\epsilon+i 0)$. The hopping parameter is assumed to be independent of the site indices and non-vanishing only between nearest neighbors, $t_{i j}=t$. The local Green's function can be written as [89]

$$
G_{00}^{-1}(\zeta)=\zeta-t^{2} \sum_{i \in N N} G_{i i}^{(0)}(\zeta)
$$

$G^{(0)}$ is the Green's function for the lattice, where site 0 is removed. But for infinite coordination number $G_{i i}^{(0)}(\zeta)=G_{i i}(\zeta)=G_{00}(\zeta)$ holds and is independent of the lattice site. Performing the appropriate scaling $t=: \frac{t^{\star}}{\sqrt{z}}$ yields $G_{00}^{-1}(\zeta)=\zeta-\left(t^{\star}\right)^{2} G_{00}(\zeta)$. One can now simply solve for $G:=G_{00}$, obtaining

$$
\begin{aligned}
G(\zeta) & =\frac{1}{2\left(t^{\star}\right)^{2}}\left(\zeta+\sqrt{\zeta^{2}-4\left(t^{\star}\right)^{2}}\right) \\
\rho(\omega) & =\frac{1}{2 \pi\left(t^{\star}\right)^{2}} \sqrt{4\left(t^{\star}\right)^{2}-\omega^{2}} .
\end{aligned}
$$

Thus the DOS of the Bethe lattice (2.5) with nearest neighbor hopping only is a semi-ellipse. One should note, that the Hilbert transformation from equation (2.2) can be performed by inserting the argument into the local Green's function $(2.5)$.

\subsubsection{Bethe lattice with next nearest neighbor hopping}

DMFT calculations depend sensitively on the DOS. The shape of the DOS can be tuned by introducing next nearest neighbor hopping, as shown in figure 2.3. I will now derive the DOS for the Bethe lattice with nearest neighbor (NN) and next nearest neighbor (NNN) hopping. I will call the hopping operator between NN-sites $H_{1}$ and the hopping operator between NNN-sites $H_{2}$. One can express the NNN-hopping term through the NN-hopping term. In infinite dimensions one obtains $[90,91]$

$$
H_{2}=\left(H_{1}\right)^{2}-1
$$



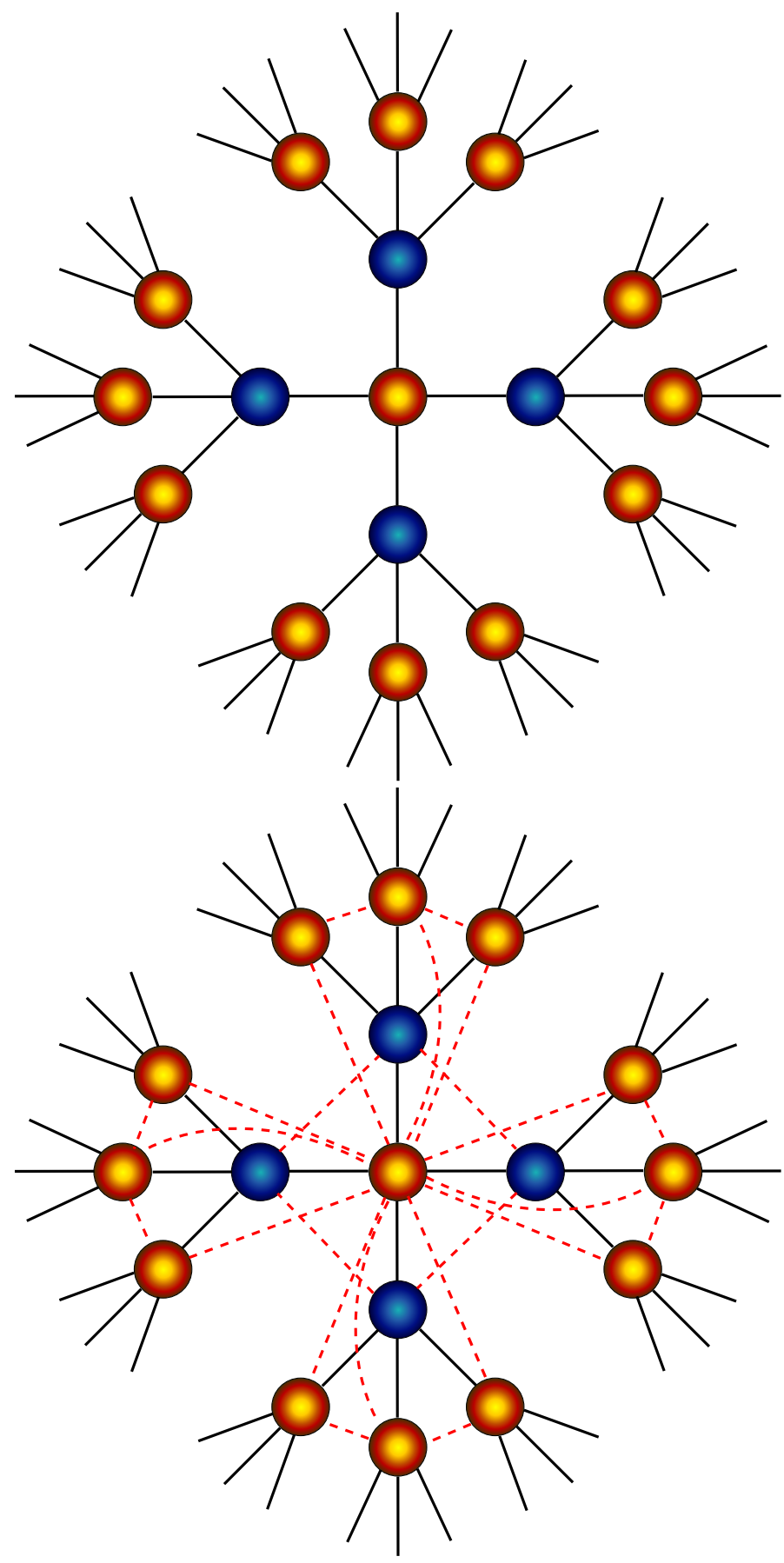

Figure 2.2: Upper panel: Part of the Bethe lattice with coordination number $z=4$. The colors reveal the bipartite structure of the lattice. Lower panel: Bethe lattice including next nearest neighbor bonds. In the lower panel it becomes apparent that the next nearest neighbor bonds act between sites with the same color (sites of the same sublattice). This introduces frustration to the Néel-state. 
This equation states that the eigenstates of the Hamiltonian $H=H_{1}+H_{2}$ equal that of $H_{1}$ alone. Let $t_{1}$ be the amplitude for the NN-hopping and $t_{2}$ for the NNN-hopping. The local Green's function can thus be evaluated to $[90,91]$

$$
\begin{aligned}
G_{t_{1}, t_{2}}(\zeta) & =\left\langle 0\left|\frac{1}{\zeta-H}\right| 0\right\rangle \\
& =\int d \epsilon \frac{\rho(\epsilon)}{\zeta-t_{1} \epsilon-t_{2}\left(\epsilon^{2}-1\right)} \\
& =\frac{1}{2 t_{2} b(\zeta)}[G(a+b(\zeta))-G(a-b(\zeta))] \\
\rho(\epsilon) & =\frac{1}{2 \pi} \sqrt{4-\epsilon^{2}} \\
a & =\frac{-t_{1}}{2 t_{2}} \\
b(\zeta) & =\sqrt{\zeta / t_{2}+a^{2}+1} .
\end{aligned}
$$

The third line in equation (2.6) was gained by partial fraction decomposition of the second line. $G(\zeta)$ (without subscript) represents the Green's function for $t_{2}=0$ given by equation (2.5). The DOS of the system without and with

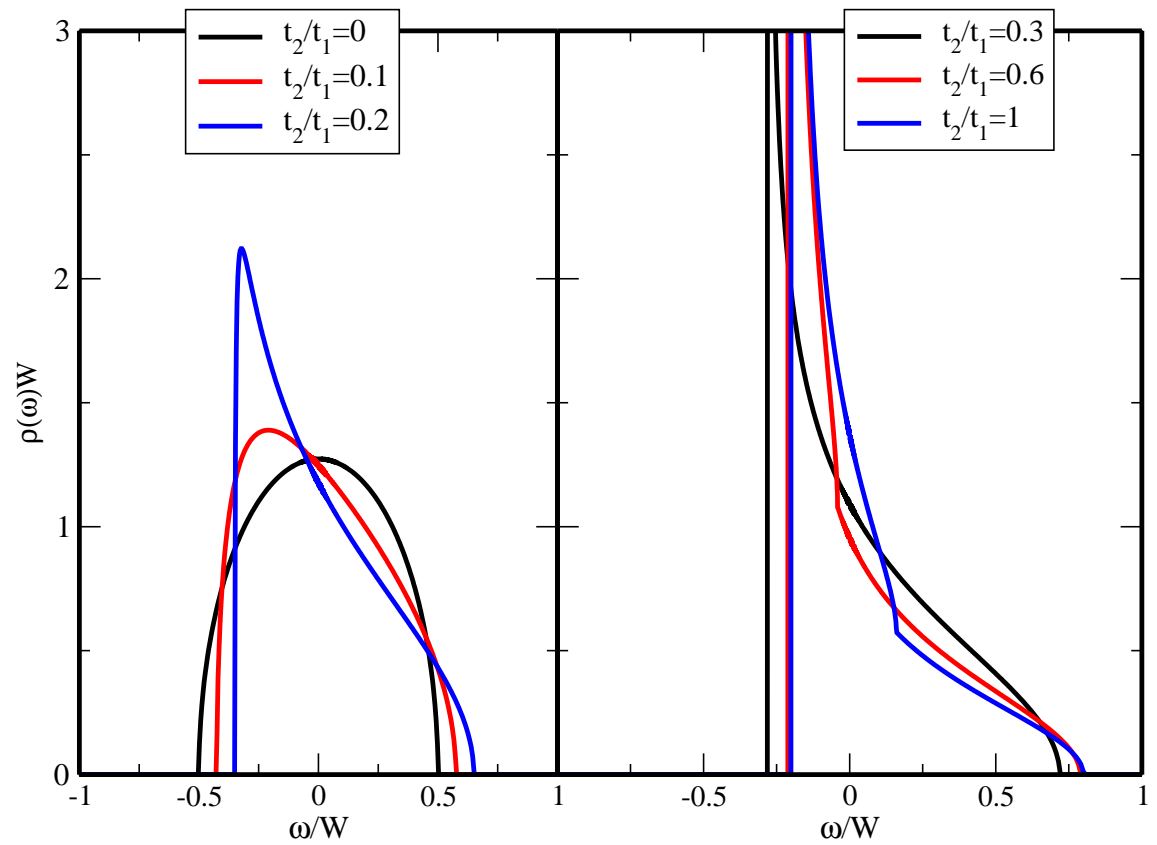

Figure 2.3: DOS in infinite dimensions for the Bethe lattice with NN-hopping $t_{1}$ and NNN-hopping $t_{2}$. The left panel shows the situation for $\frac{t_{2}}{t_{1}}<\frac{1}{4}$, in which no singularity is present. The right panel shows $\frac{t_{2}}{t_{1}} \geq \frac{1}{4}$ with a singularity at the lower band edge. The functions are normalized with the bandwidth $W$.

next nearest neighbor hopping can be seen in figure 2.3. The black curve in the left panel shows the semi-elliptic DOS of the Bethe lattice with NN-hopping only, while the other curves correspond to a finite $t_{2}>0$. When examining the 

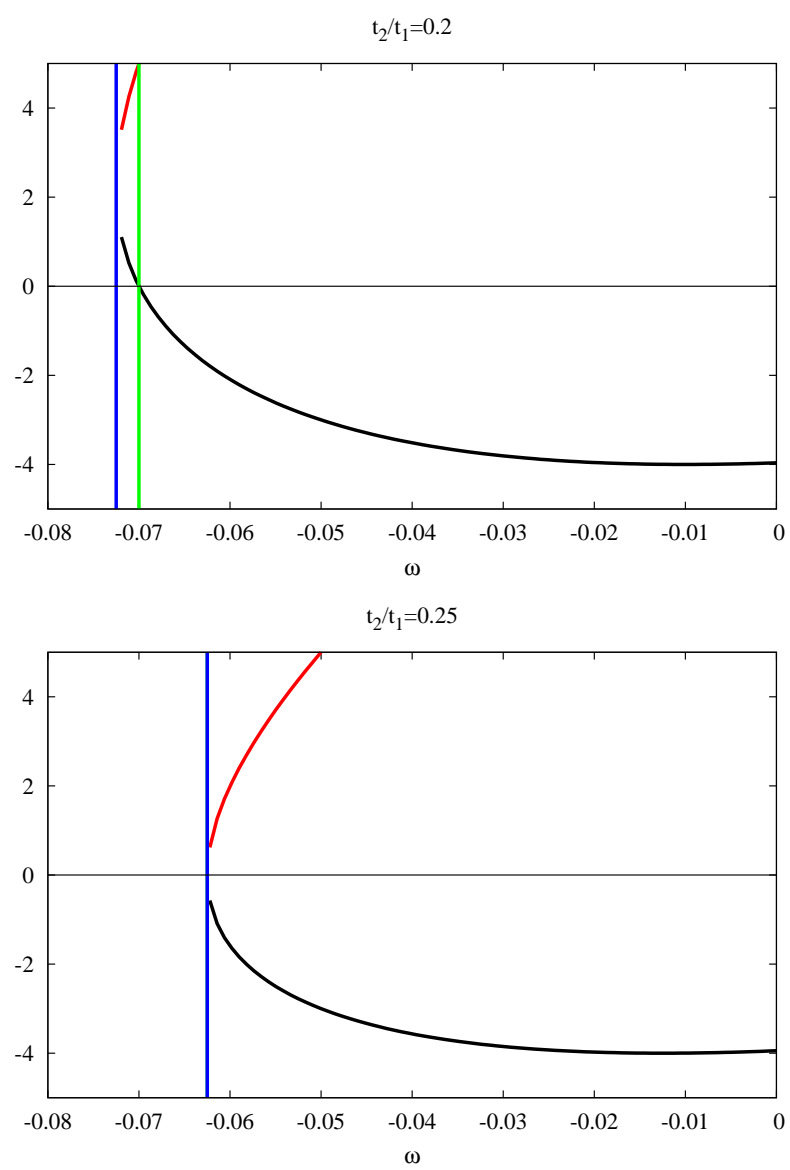

$\mathrm{t}_{2} / \mathrm{t}_{1}=0.3$

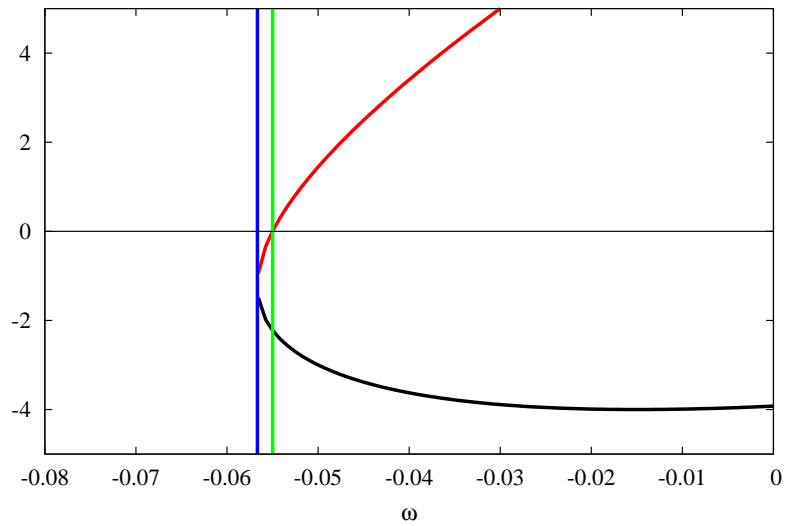

Figure 2.4: The Green's function for NN- and NNN-hopping is given by a sum of two Green's function for NN-hopping only. These three plots show the arguments of the NN-Green's function for different values of NNN-hopping. (See also the text for explanation) The black and the red curves are $(a+b(\omega))^{2}-4$ and $(a-b(\omega))^{2}-4$, respectively. The NN-hopping is in all three panels $t_{1}=0.05$. The blue vertical line represents the singularity at $\omega_{3}$ while the green vertical line represents the position of the roots of both functions at $\omega_{2}$. 
spectral function, $\rho(\omega)=-\frac{1}{\pi} \mathfrak{I m} G_{t_{1}, t_{2}}(\omega+i 0)$, one finds the following three key values for $\omega$

$$
\begin{aligned}
& \omega_{1}=3 t_{2}+2 t_{1} \\
& \omega_{2}=3 t_{2}-2 t_{1} \\
& \omega_{3}=-\frac{t_{1}^{2}}{4 t_{2}}-t_{2} .
\end{aligned}
$$

Equation (2.6) has only a non-vanishing imaginary part, if $(a+b(\omega))^{2}-4<0$ or $(a-b(\omega))^{2}-4<0$ holds, which are the square root arguments of the Green's function for NN-hopping entering. Both functions can be seen in figure 2.4. The frequency $\omega_{1}$ is the upper frequency at which the imaginary part of equation (2.6) vanishes. This means that $\rho(\omega)$ is zero for $\omega>\omega_{1}$. The vertical lines in figure 2.4 represent $\omega_{2}$ and $\omega_{3}$. At frequency $\omega_{2}$ there is a root in the arguments. For $\frac{t_{2}}{t_{1}}<\frac{1}{4}$ (upper panel in figure 2.4) both functions are positive for $\omega<\omega_{2}$ resulting in a vanishing imaginary part of equation (2.6) and thus vanishing spectral function. Frequency $\omega_{3}$ is the lower end of both functions. For $\omega<\omega_{3}$ both functions become imaginary, which does not lead to a finite contribution to the spectral function, as it cancels out. For $\frac{t_{2}}{t_{1}}>\frac{1}{4}$ both functions are shifted to negative values and there is always a negative argument for $\omega_{3} \leq \omega \leq \omega_{2}$. Thus the lower band edges of the spectral function is given by $\omega_{2}\left(\omega_{3}\right)$ for $\frac{t_{2}}{t_{1}}<\frac{1}{4}$ $\left(\frac{t_{2}}{t_{1}}>\frac{1}{4}\right)$. The frequency $\omega_{3}$ is exactly the point where $b\left(\omega_{3}\right)=0$. Looking in equation (2.6) one sees that there is a $\frac{1}{b(\omega)}$-term resulting in a square-root singularity for $\frac{t_{2}}{t_{1}}>\frac{1}{4}$ at $\omega=\omega_{3}$. This can be seen in figure 2.3. In the left panel the situation $\frac{t_{2}}{t_{1}}<\frac{1}{4}$ is plotted. There is no singularity. In the right panel there is always a singularity at the lower band edge, exactly at $\omega=\omega_{3}$. From the considerations one can derive the bandwidth of the DOS to be

$$
W=\left\{\begin{array}{ll}
4 t_{1} & t_{2} / t_{1}<1 / 4 \\
2 t_{1}+4 t_{2}+t_{1}^{2} /\left(4 t_{2}\right) & t_{2} / t_{1}>1 / 4
\end{array} .\right.
$$

In the case of next nearest neighbor hopping antiferromagnetic calculations are possible, too. The hopping Hamiltonian entering in equation (2.4) now reads $[90,91]$

$$
\mathbf{H}_{\mathbf{T}}=\left(\begin{array}{cc}
t_{2}\left(\epsilon^{2}-1\right) & t_{1} \epsilon \\
t_{1} \epsilon & t_{2}\left(\epsilon^{2}-1\right)
\end{array}\right) .
$$

As I will show later, the asymmetry and especially the singularity have great influence on the DMFT calculations. Introducing the next nearest neighbor hopping leads to frustration of the antiferromagnetic Néel-state and thus to completely different magnetic ground states. 
CHAPTER 3

\section{Impurity Solver - Theoretical Background}

\subsection{The Anderson Impurity Model}

In the last chapter I derived an effective action for the Hubbard model within DMFT. This action consists of only one interacting site coupled to non-interacting electrons in a bath, which has to be solved self-consistently. In this chapter I will introduce the single impurity Anderson model [92], which is strongly related to this effective action and discuss two strategies, how one can solve this model numerically. In this work I have in particular used two matrix based renormalization group techniques. One is the Numerical Renormalization Group (NRG) [93,94], the other is the Density Matrix Renormalization Group (DMRG) $[95,96]$. I will explain, how both methods work and what their advantages and disadvantages are.

The Anderson impurity model describes a magnetic atom embedded into a conduction band. It can be written as

$$
\begin{aligned}
H_{A n d}= & \sum_{\vec{k}, \sigma} \epsilon_{\vec{k}} c_{\vec{k}, \sigma}^{\dagger} c_{\vec{k}, \sigma}+\sum_{\sigma} \epsilon_{d} c_{d, \sigma}^{\dagger} c_{d, \sigma}+U c_{d, \uparrow}^{\dagger} c_{d, \uparrow} c_{d, \downarrow}^{\dagger} c_{d, \downarrow} \\
& +\sum_{\vec{k}, \sigma} V_{\vec{k}}\left(c_{\vec{k}, \sigma}^{\dagger} c_{d, \sigma}+c_{d, \sigma}^{\dagger} c_{\vec{k}, \sigma}\right)
\end{aligned}
$$

where $c_{\vec{k}, \sigma}^{\dagger}\left(c_{\vec{k}, \sigma}\right)$ creates (annihilates) an electron in the conduction band with quantum number $(\vec{k}, \sigma)$ and energy $\epsilon_{\vec{k}}$. In this Hamiltonian $\vec{k}$ represents a three-dimensional momentum vector. The impurity itself is represented by the operators $c_{d, \sigma}^{\dagger}\left(c_{d, \sigma}\right)$, where $\epsilon_{d}$ is the energy level and $U$ the amplitude for a 
density-density interaction on the impurity. Finally, there is a hybridization between the impurity and the conduction band given by $V_{\vec{k}}$. The Anderson model, and related with it the Kondo model [97-99], became famous for describing the physics of the Kondo problem. This effect occurs, when the resistivity of, for example, gold with embedded cobalt atoms is measured [100]. For low temperatures an unusual increase of the resistivity is found. This physical situation can be correctly described and understood with Hamiltonian (3.1) [93,98].

The Anderson Hamiltonian is most often simplified by expanding the conduction operators into spherical harmonics and assuming that only the s-wave states are coupled to the impurity level $[93,94,101,102]$. Thus the Hamiltonian can be written in the following form

$$
\begin{array}{r}
H_{A n d}=\sum_{\sigma} \int_{-D}^{D} d \epsilon \epsilon c_{\epsilon, \sigma}^{\dagger} c_{\epsilon, \sigma}+\sum_{\sigma} \epsilon_{d} c_{d, \sigma}^{\dagger} c_{d, \sigma}+U c_{d, \uparrow}^{\dagger} c_{d, \uparrow} c_{d, \downarrow}^{\dagger} c_{d, \downarrow} \\
+\sum_{\sigma} \int_{-D}^{D} d \epsilon V(\epsilon) \sqrt{\rho(\epsilon)}\left(c_{\epsilon, \sigma}^{\dagger} c_{d, \sigma}+c_{d, \sigma}^{\dagger} c_{\epsilon, \sigma}\right) .
\end{array}
$$

The s-wave band of the conduction electrons is here assumed to have energies between $-D$ and $D$. The Hamiltonian (3.2) can be visualized as in figure 3.1.

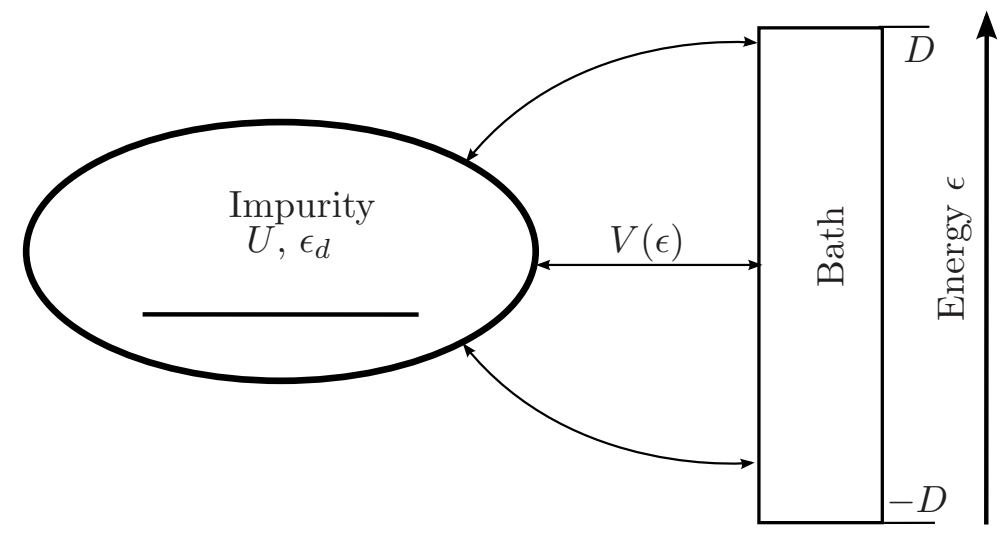

Figure 3.1: Visualization of the Anderson model. An impurity site, with parameters $U$ and $\epsilon_{d}$, is coupled via a hybridization to a bath of non-interacting electrons.

In equation (3.2) I can formally integrate out the non-interacting conduction band, deriving an effective action for the impurity level [103]

$$
S_{\text {eff,And }}=\sum_{i \omega_{n}} \sum_{\sigma} c_{d, \sigma}^{\dagger}\left(i \omega_{n}+\epsilon_{d}+\int d \epsilon \frac{V(\epsilon)^{2} \rho(\epsilon)}{i \omega_{n}-\epsilon}\right) c_{d, \sigma}+U c_{d, \uparrow}^{\dagger} c_{d, \uparrow} c_{d, \downarrow}^{\dagger} c_{d, \downarrow}
$$

where all operators turned into Grassmann variables. This action (3.3) has the same form as the effective DMFT action, see chapter 2.3. I should mention here that $\rho(\epsilon)$ is different to the density of states of the lattice in the DMFT. It is the DOS of the bath in equation (3.2), generated by the dispersion $\epsilon_{\vec{k}}$. I will now define the coupling between the impurity level and the conduction band as $h(\epsilon):=V(\epsilon) \sqrt{\rho(\epsilon)}$. Comparing with the effective action in the DMFT, one 
can identify

$$
\mathcal{G}_{0}^{-1}\left(i \omega_{n}\right)=i \omega_{n}+\epsilon_{d}+\int d \epsilon \frac{h(\epsilon)^{2}}{i \omega_{n}-\epsilon} .
$$

As $\mathcal{G}_{0}$ is an analytic function in the upper and lower complex plane, one can solve for the coupling

$$
h(\epsilon)=\sqrt{-\frac{\mathfrak{I m} \mathcal{G}_{0}^{-1}(\epsilon+i 0)}{\pi} .}
$$

With equation (3.4), I related the DMFT self-consistency loop with the Anderson model. The Anderson model is a fully quantum mechanical many body problem and there is no exact solution for calculating dynamical properties. Nevertheless, for getting an approximate solution for the Anderson model a bundle of techniques are available [98].

I will introduce matrix based renormalization group techniques in the next section. They are completely in the spirit of the renormalization group in statistical mechanics for critical behavior. For example, in the block spin renormalization [93] one replaces small clusters of spins with only one new spin representing the whole small cluster. Doing this iteratively one can look for fixed points in this calculation, which describe the physics in the thermodynamic limit.

A similar principle is used in the NRG/DMRG. Here, one starts with only a very small part of the whole problem, which can be diagonalized exactly. Then, by truncating the already diagonalized part and adding new degrees of freedom and repeated diagonalization, one can calculate iteratively an approximation for the complete system.

\subsection{Discretization of the Band States}

Before one can apply NRG/DMRG as impurity solver one has to rewrite the Hamiltonian (3.2) as an one-dimensional chain of non-interacting sites coupled to one interacting site (see figure 3.2). The Anderson Hamiltonian can be

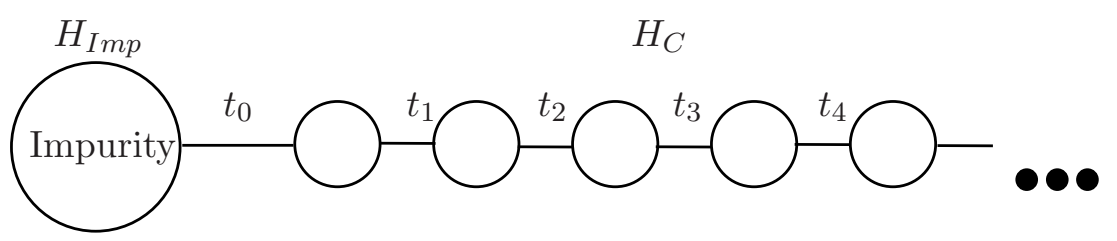

Figure 3.2: Single impurity Anderson model in form of a chain. The impurity site is unchanged. The non-interacting bath is rewritten to a semi-infinite chain with nearest neighbor hopping. $H_{I m p}$ and $H_{C}$ correspond to equation (3.5).

written as

$$
\begin{aligned}
H_{\text {And }} & =H_{\text {Imp }}+H_{c} \\
H_{\text {Imp }} & =\sum_{\sigma} \epsilon_{d} c_{d, \sigma}^{\dagger} c_{d, \sigma}+U c_{d, \uparrow}^{\dagger} c_{d, \uparrow} c_{d, \downarrow}^{\dagger} c_{d, \downarrow} \\
H_{C} & =\sum_{\sigma} \int_{-1}^{1} d \epsilon \epsilon c_{\epsilon, \sigma}^{\dagger} c_{\epsilon, \sigma}+\sum_{\sigma} \int_{-1}^{1} d \epsilon h(\epsilon)\left(c_{\epsilon, \sigma}^{\dagger} c_{d, \sigma}+c_{d, \sigma}^{\dagger} c_{\epsilon, \sigma}\right),
\end{aligned}
$$


where the conduction band energies have been scaled so that $D=1$. I will now rewrite the integral in $H_{C}$ as a discrete sum. For this purpose the interval $[-1,1]$ has been divided into disjoint intervals:

$$
[-1,1]=\bigcup_{i}\left[\omega_{l, i}, \omega_{u, i}\right]
$$

On each interval an orthonormal basis $\Psi_{i, k}:\left[\omega_{l, i}, \omega_{u, i}\right] \longrightarrow \mathbb{R}$ is set up so that $\int d \epsilon \Psi_{i, k}(\epsilon) \Psi_{i^{\prime}, k^{\prime}}(\epsilon)=\delta_{i, i^{\prime}} \delta_{k, k^{\prime}}$. One can expand a function within this basis set. For $\Psi_{i, k}$, one can use normalized Legendre polynomials or a local Fourier basis. With these functions, one defines new operators

$$
\begin{aligned}
c_{i, k, \sigma} & =\int d \epsilon c_{\epsilon, \sigma} \Psi_{i, k}(\epsilon) \\
c_{\epsilon, \sigma} & =\sum_{i, k} c_{i, k, \sigma} \Psi_{i, k}(\epsilon) .
\end{aligned}
$$

These operators follow the usual anti-commutator relations, as the basis is orthonormal $[93,101,102]$. One can now rewrite (3.5)

$$
\begin{aligned}
H_{c} & =\sum_{k, k^{\prime}, i, \sigma} \epsilon_{i, k, k^{\prime}} c_{i, k, \sigma}^{\dagger} c_{i, k^{\prime}, \sigma}+\sum_{k, i, \sigma} h_{i, k}\left(c_{i, k, \sigma}^{\dagger} c_{d, \sigma}+c_{d, \sigma}^{\dagger} c_{i, k, \sigma}\right) \\
\epsilon_{i, k, k^{\prime}} & =\int d \epsilon \epsilon \Psi_{i, k}(\epsilon) \Psi_{i, k^{\prime}}(\epsilon) \\
h_{i, k} & =\int d \epsilon h(\epsilon) \Psi_{i, k}(\epsilon) .
\end{aligned}
$$

Analyzing equation (3.7), one can see, that each operator $c_{i, k, \sigma}$ couples to the impurity $c_{d, \sigma}$. This kind of Hamiltonian is usually referred to as star geometry. For real calculations, one has to truncate the orthonormal basis set to only a few basis functions. This must be done for assuring that the number of sites is finite. Finally, one can tridiagonalize $H_{C}$ (3.7) with a Householder algorithm [104] bringing it into chain geometry, as can be seen in figure 3.2.

\subsection{Numerical Renormalization Group}

\subsubsection{Discretization within the NRG}

The Numerical Renormalization Group (NRG) was developed by Kenneth Wilson [93] in order to explain the Kondo effect. Some years later the method was applied to the Single Impurity Anderson Hamiltonian by H. KrishnaMurthy $[101,102]$. An overview of this method and recent developments can be found in R. Bulla et al. [94].

The main point in the NRG algorithm is that one has to use a logarithmic mesh for the discretization of the conduction band. The reason for this will become clear below. Hence, the intervals have the form

$$
\begin{aligned}
I_{n, \pm} & =\left[ \pm \Lambda^{-(n+1)}, \pm \Lambda^{-n}\right] \quad n \in \mathbb{N} \\
L_{n} & =\left|\Lambda^{-(n+1)}-\Lambda^{-n}\right| .
\end{aligned}
$$


The constant $\Lambda>1$ is a free parameter, setting the logarithmic discretization of the intervals. The goal of the logarithmic discretization is to have a very good resolution, meaning very small intervals, near the Fermi energy $\epsilon=0$. Usually, one uses values for the discretization of approximately $\Lambda \approx 2$. As described above, one sets up an orthonormal basis within each interval. For NRG calculations one neglects all terms but the constant function in each interval. It can be shown that the coupling between the different orthonormal functions is small [93]. Nevertheless, it is a rather rude approximation, especially when using $\Lambda=2$, which is only justified a posteriori by the results gained $[93,94,101,102]$. On the other hand, using only the constant function on each interval, it can be shown that the solution is exact for $\Lambda \rightarrow 1$. So it is recommendable always to check the results by doing a calculation with smaller $\Lambda$. If nothing changes, one can assume to have a good approximation.

One can now transform the conduction band Hamiltonian $H_{c}$ into the form of a linear chain with the unchanged impurity at the beginning of the chain (see figure 3.2). The important point of the logarithmic discretization and this mapping is that the coupling constants $t_{i}$ decrease exponentially along the chain. This exponential decrease is of great importance, as the iterative diagonalization is doomed to fail, if this property is not given. I will explain this in more detail in the next section.

In real calculations the chain is cut after $N$-sites. For NRG-calculations typical chain lengths are of the order of $N=15-100$ sites. Although $N=15$ sites sounds very few, as the degrees of freedom grow exponentially, there are already

$$
4^{15}=1073741824
$$

degrees of freedom. This number is too large for a direct diagonalization, so one is forced to use iterative diagonalization schemes.

\subsubsection{Iterative diagonalization}

The iterative diagonalization procedure of this semi-infinite chain is visualized in figure 3.3. One begins with setting up the complete Fock space for the impurity site. As the number of states is small, it can be diagonalized completely. Taking the new eigenstates one can set up the basis for the impurity coupled to the first site of the chain. The new Fock space is just a tensor product of the eigenstates of the impurity and a Fock space basis for the first site. Diagonalizing this small cluster again yields the eigenstates, which are used to set up the new basis including the next site. This is done iteratively as can be seen in figure 3.3. The problem is that the number of states in the Fock space grows exponentially. For a complete diagonalization one is usually limited to about three or four sites of the lattice. In order to continue one has to truncate the Fock space. In NRG calculation one takes the $N_{K}$ energetic lowest Fock space states. Only these are used to set up the new Fock space including the next site. As a result, one can continue to iteratively diagonalize the chain always truncating the space to the energetic lowest $N_{K}$ states. The reason why this truncation scheme works here is that the coupling of the next site to the already diagonalized part of the chain is weak compared to the current energetic scale. The coupling between 


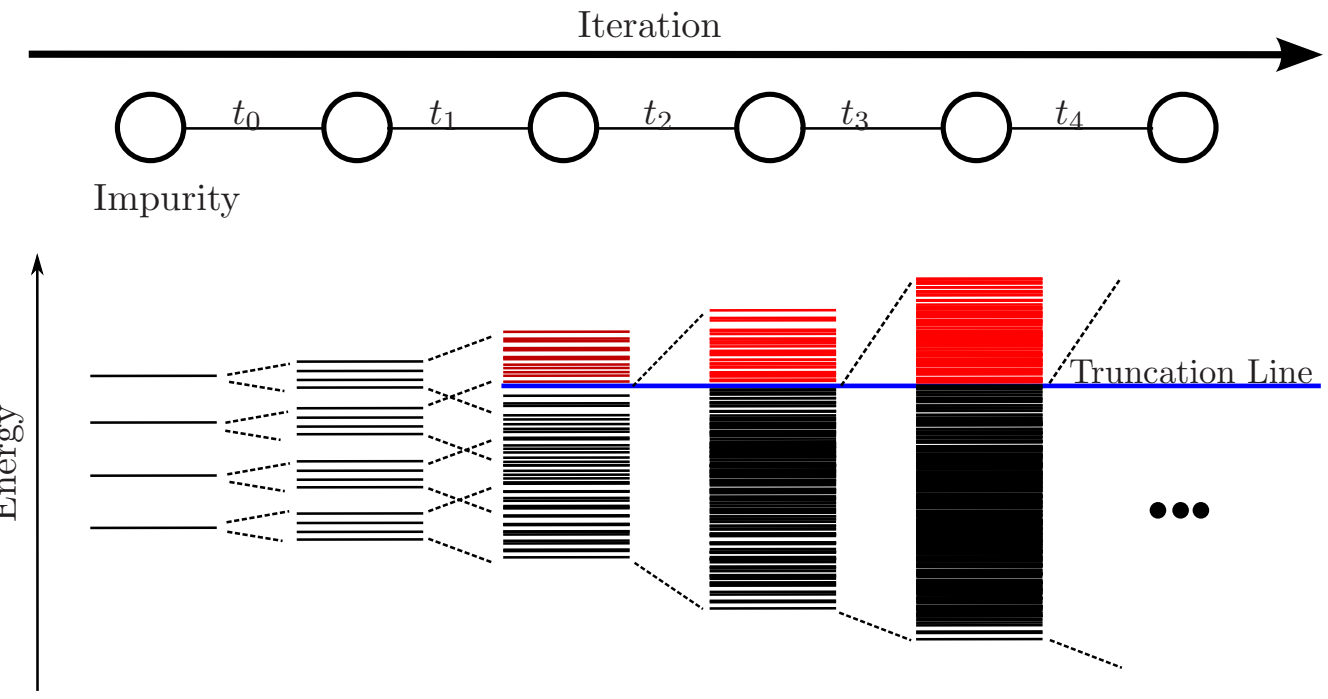

Figure 3.3: Iterative diagonalization procedure in the NRG. See the text for description.

the states should be small compared to the difference between the energetic lowest and highest state. Loosely speaking, the next site is supposed to be only a small perturbation for the already diagonalized chain. This is ensured by the exponential decrease of the hopping amplitude $t_{i}$, which comes from the logarithmic discretization of the bath states in the beginning. This iteration procedure can be written as

$$
\begin{aligned}
H_{N}= & \Lambda^{(N-1) / 2}\left(H_{\text {Imp }}+t_{0} \sum_{\sigma}\left(c_{d, \sigma}^{\dagger} c_{0, \sigma}+c_{0, \sigma}^{\dagger} c_{d, \sigma}\right)\right. \\
& \left.+\sum_{i=0, \sigma}^{N} \epsilon_{i} c_{i, \sigma}^{\dagger} c_{i, \sigma}+\sum_{i=0, \sigma}^{N-1} t_{i+1}\left(c_{i, \sigma}^{\dagger} c_{i+1, \sigma}+c_{i+1, \sigma}^{\dagger} c_{i, \sigma}\right)\right) \\
H_{N+1}= & \sqrt{\Lambda} H_{N}+\Lambda^{N / 2} \epsilon_{N+1} \sum_{\sigma} c_{N+1, \sigma}^{\dagger} c_{N+1, \sigma} \\
& +\Lambda^{N / 2} t_{N+1} \sum_{\sigma}\left(c_{N, \sigma}^{\dagger} c_{N+1, \sigma}+c_{N+1, \sigma}^{\dagger} c_{N, \sigma}\right) \\
H= & \lim _{N \rightarrow \infty} \Lambda^{-(N-1) / 2} H_{N} .
\end{aligned}
$$

This defines a renormalization group transformation $R$ [93,101,102]

$$
H_{N+1}=R\left[H_{N}\right],
$$

which transforms a Hamiltonian $H_{N}$ into another Hamiltonian $H_{N+1}$. The prefactor $\Lambda^{(N-1) / 2}$ has been introduced in equation (3.8) to compensate the decreasing of the hopping parameters $t_{i}$. Thus, the energy levels remain of the same order of magnitude ensuring a constant numerical diagonalization error. Even if one is able to continue iteratively diagonalizing the semi-infinite chain, one has to stop at some point. There are two criteria when to stop the calculation. The first criterion is given when the calculation has reached a fixed point, 
meaning, that the energy levels of $H_{N}$ equal $H_{N+2}$ and do not change anymore. The energy levels may change from even to odd iterations even when the fixed point is reached. When the zero temperature fixed point is reached, one can stop the iterative procedure and one is able to calculate zero temperature properties. Care must be taken, as also meta-stable fixed points may exist. The other criterion for stopping the procedure applies if one is interested in finite temperature results. As the coupling decreases exponentially, also the imposed energy scale decreases. At some point the changing of the energy levels will be smaller than the energy scale defined by the temperature. The changes in the position of the energy levels by further diagonalizing the chain will not change the Boltzmann weights in the calculation of thermodynamical properties for the given temperature anymore. This is the point when one can stop the diagonalization of the chain. Further diagonalization will not change the finite temperature results.

\subsubsection{Calculation of impurity properties}

The calculation of static expectation values on the impurity is straight forward [94]. One simply sets up the matrix for the corresponding operator at the beginning of the iterative diagonalization. This matrix is always updated during the procedure into the current basis. Using the energy values and the corresponding Boltzmann weights one is able to calculate thermodynamic static expectation values. Let $A$ be the operator of interest, $\beta=\frac{1}{k_{B} T}$ the inverse temperature, $Z$ the partition function, and $|i\rangle$ a state from the current basis with energy $E_{i}$. Then the expectation value reads

$$
\langle A\rangle=\frac{1}{Z} \sum_{i} \exp \left(-\beta E_{i}\right)\langle i|A| i\rangle .
$$

In this formula the sum is supposed to run over a complete basis set, which is difficult to determine, as the basis is truncated during the iterative diagonalization. For thermodynamic properties it is often a very good approximation to assume that the only relevant contributions for a given temperature come from the chain with the length, where the temperature equals approximately the characteristic energy scale of the last site [94]. A more sophisticated approach will be shown below.

The calculation of dynamical properties is a little bit more subtle. At the end of the calculation one is able to calculate static expectation values and has found the eigenstates and the corresponding energies. Thus one is able to use Lehmann's formula to calculate spectral functions for an operator $A$. The retarded single particle Green's function for fermionic operators is given by

$$
G_{A B}(t)=-i \Theta(t) \mathcal{T} \rho[A(t) B(0)+B(0) A(t)] .
$$

If there were no truncation during the diagonalization, using Laplace transformation, one would obtain

$$
G_{A B}(\zeta)=\frac{1}{Z} \sum_{i, j}\langle i|A| j\rangle\langle j|B| i\rangle \frac{\exp \left(-\beta E_{i}\right)+\exp \left(-\beta E_{j}\right)}{\zeta+E_{i}-E_{j}},
$$


where $\rho=\exp (-\beta H) / Z$ was inserted. Furthermore, the partition function is given by $Z=\sum_{i} \exp \left(-\beta E_{i}\right)$, and $\zeta$ is a complex variable. The spectral function obtained by this reads

$$
\rho_{A B}(\omega)=\frac{1}{Z} \sum_{i, j}\langle i|A| j\rangle\langle j|B| i\rangle\left(\exp \left(-\beta E_{i}\right)+\exp \left(-\beta E_{j}\right)\right) \delta\left(\omega+E_{i}-E_{j}\right) .
$$

The problematic point in this formula is that the sum over $i, j$ must be over a complete basis set. If one uses this formula only with the basis set of the last iteration, one will use only a very tiny fraction of a complete basis due to the truncation during the iterative diagonalization. Consequently, the calculated spectral function will contain only frequency points very close to $\omega=0$. All high energy features will be lost. But adding all iterations of the chain will give an over-complete basis. It will include a double counting of some states, which must be accounted for by some regulation process $[105,106]$. This regulation procedure is in some way arbitrary and introduces a new and uncontrolled approximation.

To overcome this regulation one has to identify a complete basis set. The idea, how to do this in the NRG chain, was by F. Anders and A. Schiller [107, 108]. It was tested for equilibrium Green's functions by RP, T. Pruschke and F. Anders [109] and separately by A. Weichselbaum and J. von Delft [110]. The new approach does focus on a Wilson chain of length $N$. In the beginning of the iteration procedure a complete basis can be simply identified as a tensor product of all single site Fock space states. Before the first truncation in the iterative diagonalization procedure at site $m<N$, the eigenstates of the diagonalization $\Psi_{k_{m}}^{m}$ yield a complete basis set of the chain of site $m$. If one performs a tensor product with a basis for the rest of the chain $m<k \leq N$,

$$
\Psi_{k_{m}}^{m} \otimes \psi_{k_{m+1}}^{m+1} \otimes \ldots \otimes \psi_{k_{N}}^{N}
$$

one still obtains a complete basis set. Here $\Psi_{k_{m}}^{m}$ is an eigenstate of the chain of length $m$ and $\psi_{k_{m}+1}^{m+1}$ a basis state of the single site $m+1$. If one now truncates the chain at length $m$, one neglects these states times a complete basis for the rest of the chain, when trying to build up a complete basis set. The part of the basis, which is created by the kept states, the states which are not truncated, is not changed by the diagonalization. The kept states will be changed in the diagonalization of the chain of length $m+1$. But a tensor product of all states in $m+1$ with a complete basis for the Fock space for $m+1<k \leq N$ spans the same space as the kept states at length $m$ times a basis for the Fock space $m<k \leq N$ :

$$
\begin{aligned}
& \operatorname{Span}\left(\sum_{k_{m} \in \mathrm{kept}} \sum_{k_{m+1}, \ldots, k_{N}} \Psi_{k_{m}}^{m} \otimes \psi_{k_{m+1}}^{m+1} \otimes \ldots \otimes \psi_{k_{N}}^{N}\right)= \\
& \operatorname{Span}\left(\sum_{k_{m+1}} \sum_{k_{m+2}, \ldots, k_{N}} \Psi_{k_{m+1}}^{m+1} \otimes \psi_{k_{m+2}}^{m+2} \otimes \ldots \otimes \psi_{k_{N}}^{N}\right) .
\end{aligned}
$$


Thus, one can identify a complete basis set which consists of all truncated states times a basis for the rest of the chain, where they have been truncated. In the last step of the iterative diagonalization all states are supposed to be truncated. In the following, the notation for a state shall be:

$$
|\Psi\rangle=\left|m, k_{m}, l\right\rangle=\left|m, k_{m}\right\rangle \otimes|l\rangle,
$$

where $m$ labels the chain site, $k_{m}$ is the index of the eigenstates at this site and $l$ is an index for a state in the basis for the rest of the chain. After the truncation of a state, denoted as $|\cdot\rangle_{\text {Trunc }}$, the impurity properties inherent in this state are not changed anymore as the state is decoupled from the rest of the chain

$$
{ }_{\text {Trunc }}\left\langle m, k_{m_{1}}, l_{1}\left|A_{\text {Imp }}\right| m, k_{m_{2}}, l_{2}\right\rangle_{\text {Trunc }}=\left\langle m, k_{m_{1}}\left|A_{\text {Imp }}\right| m, k_{m_{2}}\right\rangle \delta_{l_{1}, l_{2}} .
$$

Thus the environment states $|l\rangle$ do not change the expectation values of an impurity operator after the truncation. If one now inserts a complete basis set consisting only of truncated states in equation (3.10), one question must still be clarified: What is the expectation value of two states truncated at different sites $m$ and $m^{\prime}$ in the chain for $m<m^{\prime}$. A state truncated at site $m^{\prime}$ can be written as a linear combination of kept states at site $m$

$$
\left|m^{\prime}, k_{m}^{\prime}, l\right\rangle_{\text {Trunc }}=\sum_{k_{m} \in \mathrm{kept}} \sum_{l^{\prime}} \Phi_{k_{m}, l^{\prime}}\left|m, k_{m}, l^{\prime}\right\rangle
$$

where the $l^{\prime}$ is labeling environment states. $\Phi_{k_{m}, l^{\prime}}$ are coefficients in the orthogonal matrices of the diagonalization. Thus the contributions of combinations of states truncated at different shells $m<m^{\prime}$ can be accounted for. One has to take the combinations of the truncated state $k_{m}$ in shell $m$ and all the kept states in the same shell, as the environment states do not change the expectation value with an impurity operator.

Although the environment states do not change these direct expectation values with an impurity operator, they influence the value of the density operator. Their influence on it can be taken into account with the reduced density matrix $[111]$

$$
\rho_{k_{m}, k_{m}^{\prime}}^{r e d}=\sum_{l}\left\langle m, k_{m}, l|\rho| m, k_{m}^{\prime}, l\right\rangle .
$$

The contributions of a state to the static or dynamical expectation value of the impurity can be calculated correctly at the site, where the state is truncated. For calculating magnetic solutions within the NRG, it is essential to use the reduced density matrix $\rho^{\text {red }}$ [111]. A small magnetic field can strongly influence the ground state properties of an impurity calculation. Calculating dynamical properties without using the reduced density matrix did often dramatically underestimate the influence of the magnetic field.

In summary, when doing a calculation using a complete basis set, one must take all the contributions of all truncated states and all states of the last iteration. In the end this can be easily understood. All kept states are only refined in the following iterations. Thus contribution between two kept states can be taken 
into account in later iterations. Defining $|m, i\rangle$ as a state in iteration $m$, the Green's function can be written as

$$
\begin{aligned}
G_{A B}(\zeta)= & \sum_{m=0}^{N} \sum_{i, j, k}^{\prime}\left(\langle m, i|A| m, j\rangle \rho_{j, k}^{r e d}\langle m, k|B| m, i\rangle\right. \\
& \left.+\langle m, i|A| m, k\rangle \rho_{j, i}^{r e d}\langle m, k|B| m, j\rangle\right) \frac{1}{\zeta+E_{i}-E_{k}} \\
\rho_{A B}(\omega)= & \sum_{m=0}^{N} \sum_{i, j, k}^{\prime}\left(\langle m, i|A| m, j\rangle \rho_{j, k}^{r e d}\langle m, k|B| m, i\rangle\right. \\
& \left.+\langle m, i|A| m, k\rangle \rho_{j, i}^{r e d}\langle m, k|B| m, j\rangle\right) \delta\left(\omega-E_{i}+E_{k}\right),
\end{aligned}
$$

where now the summations $\sum_{i, j, k}{ }^{\prime}$ is such, that at least one of the states $|m, i\rangle$ or $|m, k\rangle$ is a truncated state. The spectral function is again given by $\rho_{A B}(\omega)=$ $-\frac{1}{\pi} \mathfrak{I m} G_{A B}(\omega+i 0)$.

This formula can now be applied to the NRG diagonalization. After diagonalization of the complete chain, one sets up the density matrix and iterates backwards from the end of the chain, setting up the reduced density matrix for each site. For each iteration one applies equation (3.11) and collects all the delta peaks for the spectral function. To gain a smooth spectrum, one has to replace the delta peaks in equation (3.11) by continuous functions. As the discretization was done on a logarithmic scale, it is quite common to use Gaussian functions on a logarithmic scale for doing this broadening [94]

$$
\delta(\omega-E) \rightarrow \frac{\exp \left(-\frac{b^{2}}{4}\right)}{b E \sqrt{\pi}} \exp \left(-(\log (|\omega| / E) / b)^{2}\right)
$$

where $b$ is a broadening parameter, whose value is typically $b \approx 0.3-0.8$. For frequencies below the smallest energetic discretization $\Lambda^{-(N-1) / 2}$ it is advisable to use Lorentzians instead of logarithmic Gaussians. The reason is that pure logarithmic Gaussians will always result in $\rho(\omega=0)=0$. As one can think of the smallest energetic discretization as a finite temperature, it is justified to use a Lorentzian here.

The same complete Fock space treatment can be used for calculating static expectation values, too [110]. One can determine the exact Boltzmann weight to each Fock space state in the complete basis set. This will lead to the situation that more than only one NRG shell contributes to thermodynamical properties. The advantage of this procedure is, that one can calculate properties at arbitrary temperature with precise Boltzmann weights.

\subsubsection{Calculating the Self-energy}

For the DMFT self-consistency equation one needs the self-energy of the impurity. The expression for the self-energy can be derived by equations of mo- 
tion $[112]$

$$
\begin{aligned}
1= & \left(\zeta-\epsilon_{d}\right) G_{c_{d, \sigma}, c_{d, \sigma}^{\dagger}}(\zeta)-U G_{c_{d, \sigma} c_{d,-\sigma}^{\dagger} c_{d,-\sigma}, c_{d, \sigma}^{\dagger}}(\zeta) \\
& -\sum_{\vec{k}} V_{\vec{k}} G_{c_{\vec{k}, \sigma}, c_{d, \sigma}^{\dagger}}(\zeta) \\
0= & \left(\zeta-\epsilon_{k}\right) G_{c_{\vec{k}, \sigma}, c_{d, \sigma}^{\dagger}}(\zeta)-V_{\vec{k}} G_{c_{d, \sigma}, c_{d, \sigma}^{\dagger}}(\zeta) \\
1= & \left(\zeta-\epsilon_{d}\right) G_{c_{d, \sigma}, c_{d, \sigma}^{\dagger}}(\zeta)-U F_{\sigma}(\zeta)-\Delta G_{c_{d, \sigma}, c_{d, \sigma}^{\dagger}}(\zeta) \\
\Delta(\zeta)= & \sum_{\vec{k}} V_{\vec{k}}^{2} \frac{1}{\zeta-\epsilon_{\vec{k}}} \\
F_{\sigma}(\zeta)= & G_{c_{d, \sigma} c_{d,-\sigma}^{\dagger} c_{d,-\sigma}, c_{d, \sigma}^{\dagger}}(\zeta),
\end{aligned}
$$

finally yielding

$$
\Rightarrow \Sigma_{\sigma}(\zeta)=U \frac{F_{\sigma}(\zeta)}{G_{c_{d, \sigma}, c_{d, \sigma}^{\dagger}}(\zeta)}
$$

with the notation of equation (3.1) and the Green's function $G_{A, B}$ for operators $A$ and $B$. Thus, the self-energy of the impurity can be calculated as a quotient of two Green's functions, which in turn can be calculated within the scheme just derived in this chapter.

\subsubsection{Two-orbital Anderson model}

In the last sections I have dealt with the single impurity Anderson model. But focusing on a two-orbital Hubbard model within DMFT, it is essential to introduce the two-orbital Anderson model, as DMFT will map the multiorbital lattice model onto a multi-orbital Anderson model. The local part of the Anderson Hamiltonian is the same as in the Hubbard model. Thus the Hamiltonian for the two-orbital Anderson model reads

$$
\begin{aligned}
H_{A n d, 2}= & H_{C}+H_{I m p} \\
H_{I m p}= & \sum_{m=1}^{2} \sum_{\sigma} \epsilon_{d} n_{d, m, \sigma}+U \sum_{m=1}^{2} n_{d, m, \uparrow} n_{d, m, \downarrow} \\
& +\left(U^{\prime}-\frac{1}{2} J\right) \sum_{\sigma, \sigma^{\prime}} n_{d, 1, \sigma} n_{d, 2, \sigma^{\prime}}-2 J \vec{S}_{d, 1} \cdot \vec{S}_{d, 2} \\
H_{C}= & \sum_{m=1}^{2} \sum_{\sigma} \int_{-D}^{D} d \epsilon \epsilon c_{\epsilon, m, \sigma}^{\dagger} c_{\epsilon, m, \sigma} \\
& +\sum_{m=1}^{2} \sum_{\sigma} \int_{-D}^{D} d \epsilon V(\epsilon) \sqrt{\rho(\epsilon)}\left(c_{\epsilon, m, \sigma}^{\dagger} c_{d, m, \sigma}+c_{d, m, \sigma}^{\dagger} c_{\epsilon, m, \sigma}\right),
\end{aligned}
$$

where each operator has an additional orbital quantum number $m$. Operators acting on the impurity are labeled with "d". This model can be mapped, as shown above, on an one-dimensional chain, where each site has a local Fock 
space consisting of 16 states due to the two degenerate conduction bands. Thus the complete Fock space grows with the number of sites $N$ as $16^{N}$. To get reasonable results for static and dynamic properties within the NRG, I kept up to 5000 states per iteration. For one complete diagonalization and the calculation of spectral functions one needs up to 16GB main memory and several processor hours computing time on a multi-core-processor. In contrast, for an one-orbital model often 1000 - 2000 states and 1GB main memory are enough. For the calculations in the two-orbital model I used three conserved quantum numbers: particle number $N$, total $S_{Z}$ of the spin components and total orbital quantum number $T_{z}$. If one would include the above mentioned pair hopping term into the Hamiltonian the orbital quantum number would not be conserved anymore. This would result in larger quantum number subspaces during the iterative diagonalization and thus larger amount of main memory and time for the computation.

\subsection{Density Matrix Renormalization Group}

I developed a new Density Matrix Renormalization Group (DMRG) code. I especially focused on solving impurity problems like the Anderson impurity model. The main reason for this development was to get some independent results with a method different from the NRG for the magnetic phase diagram in the Hubbard model. DMRG was originally designed for treating onedimensional systems $[95,96,113,114]$. But as I have shown above, an impurity model like the Anderson model can be mapped onto a one-dimensional chain [93]. In contrast to the NRG, no logarithmic discretization must be used for the mapping within the DMRG. For this method one can freely choose the intervals. This allows for more freedom and one is able to properly control the accuracy of the discretization. DMRG can then be used for diagonalizing this chain representing the Anderson impurity model [115-118].

\subsubsection{Iterative diagonalization}

Similar to the NRG, also in the DMRG the chain is diagonalized iteratively. Here too, the Fock space grows exponentially reaching very quickly a size, which cannot be diagonalized anymore in suitable time. One has to truncate the Fock space selecting a smaller number of states, which will build up the next iteration. This is done in a way best fitting the aim of the procedure. In the NRG the states with the lowest energies were selected to create the next Hamiltonian. The reason for this was the exponentially decreasing hopping parameters and the focus on the low energy eigenstates of the problem. In the DMRG the hopping parameters can have arbitrary values. Selecting the lowest eigenstates in smaller blocks for building up larger blocks fails because of wrong boundary conditions [119]. In DMRG the new states for an enlarged block are calculated using the ground state of the whole system. From the ground state one can build up the reduced density matrix [120] for the enlarged block. Let me assume the whole system is build up from four blocks, see figure 3.4. The middle blocks B2 and B3 correspond to single sites containing only a very limited number of 


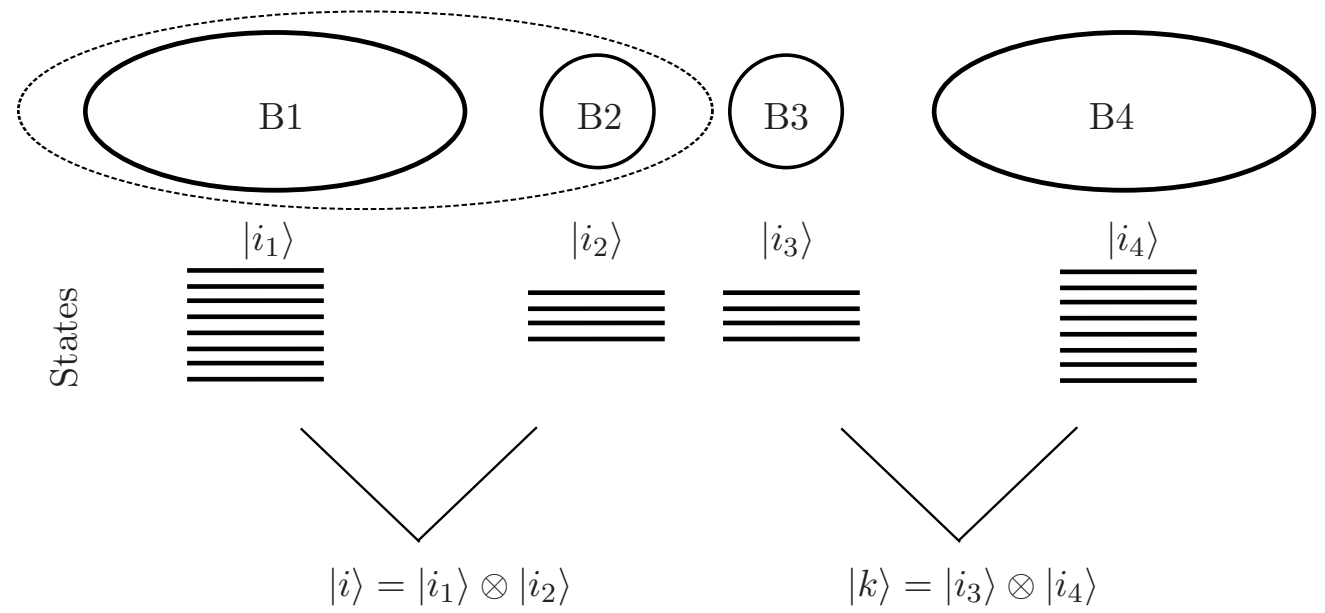

Figure 3.4: One iteration step in DMRG. The blocks are named B1, B2, B3 and B4 with states $\left|i_{1}\right\rangle,\left|i_{2}\right\rangle,\left|i_{3}\right\rangle$ and $\left|i_{4}\right\rangle$. A basis set is formed by tensor product for the two left blocks and two right blocks, respectively.

states. The remaining blocks B1 and B4 represent all sites of the left or the right part. Both are supposed to already contain a truncated Fock space. I will now explain the method how to build up the next enlarged block.

Each block consists of a finite number of states $\left|i_{k}\right\rangle$. From these states the Hamiltonian for the whole system can be set up. For this Hamiltonian one searches the ground state by the Lanczos method or some similar technique $[121,122]$. Assuming the chain is represented by 4 blocks the ground state can be written as

$$
\Psi=\sum_{i_{1}, i_{2}, i_{3}, i_{4}} \Phi_{i_{1}, i_{2}, i_{3}, i_{4}}\left|i_{1}\right\rangle \otimes\left|i_{2}\right\rangle \otimes\left|i_{3}\right\rangle \otimes\left|i_{4}\right\rangle
$$

One can now combine both left blocks forming one new block $|i\rangle=\left|i_{1}\right\rangle \otimes\left|i_{2}\right\rangle$. The same is done for the two right blocks. As a result, one can write the ground state in the states of the two big blocks

$$
\Psi=\sum_{i, k} \psi_{i, k}|i\rangle \otimes|k\rangle
$$

From the coefficients $\psi_{i, k}$ one can now form the reduced density matrix for the left blocks

$$
\rho_{i, j}=\sum_{k} \psi_{i, k} \psi_{j, k}
$$

where $i, j$ are indices of the left block, $k$ an index of the right block and $\psi_{i, k}$ is assumed to be real. This matrix $\rho_{i, j}$ is supposed to have a maximal dimension of about $\operatorname{dim} \rho_{i j} \approx 1000$, as one has to diagonalize this matrix completely in each iteration.

Let me assume that I want to calculate the expectation value of a bounded operator $\|A\|=\max _{\phi}\left|\frac{\langle\phi|A| \phi\rangle}{\langle\phi \mid \phi\rangle}\right|=c_{A}$ acting only in the left enlarged block. This expectation value is given by the trace of the operator times the density 
operator $\rho$. As the operator acts only at the left block, the density operator corresponds to equation (3.13). As $\rho$ is a symmetric matrix one can write it as $\rho=\sum_{i}\left|\lambda_{i}\right\rangle \lambda_{i}\left\langle\lambda_{i}\right|$, where the eigenvalues are supposed to be ordered from large to small. One wants to truncate the dimension of the block from $\operatorname{dim} \rho_{i j}$ to $m<\operatorname{dim} \rho_{i j}$, thus taking the eigenstates with the largest eigenvalues. The expectation value can be written as

$$
\begin{aligned}
\langle A\rangle & =\mathcal{T} r \rho A \\
& \approx \sum_{i=0}^{m} \lambda_{i}\left\langle\lambda_{i}|A| \lambda_{i}\right\rangle .
\end{aligned}
$$

The error of the truncation for this operator can be easily approximated by

$$
\left|\langle A\rangle-\sum_{i=0}^{m} \lambda_{i}\left\langle\lambda_{i}|A| \lambda_{i}\right\rangle\right|=\sum_{i=m+1}^{\operatorname{dim}} \lambda_{i} c_{A}
$$

being proportional to the sum of the neglected smallest eigenvalues. Thus the eigenstates with the largest eigenvalues of the density operator best describe the operator $A$ [113]. This holds for every bounded operator, especially for the part of the Hamiltonian describing the system of the two blocks. One can also argue that these states best describe the ground state wave function [123] or maximize the quantum entanglement [124].

The algorithm now works as follows: First one calculates the ground state of four single sites. From this a combined block including two single sites is created. So the new system consist of the combined block and three single sites from which a combined block of three sites is created. Going on like this the whole chain can be built up. After setting up the whole chain, one can use the same algorithm to improve the basis stored in each combined block by first enlarging the left blocks and later the right blocks. Iterating this procedure one can get a very accurate ground state wave function of an one-dimensional chain. With this wave function one can calculate static expectation values or correlation functions of the chain. Of course, this includes expectation values of the impurity itself.

\subsubsection{Calculation of dynamical properties}

The DMRG focuses on the ground state of the system. One usually is not able to calculate more than a very few excited states. Therefore, one is not able to use Lehmann's formula for calculating spectral functions. The easiest way calculating a spectral function is using a Lanczos expansion of the spectral function $[125,126]$ calculating the first few moments. But this expansion fails for more complex dynamical spectral functions.

An alternative approach is the correction-vector method $[126,127]$. A very elegant way of formulating it, also improving its accuracy, was introduced by E. Jeckelmann [128]. The Laplace transformed retarded Green's function (3.10) for $T=0$ can be written as

$$
G_{A B}(\zeta)=\left\langle\Psi\left|A \frac{1}{\zeta-H} B\right| \Psi\right\rangle
$$


where $\Psi$ is the ground state of the chain. For numerical calculation it is necessary that $\zeta=\omega+i \eta$ has positive finite imaginary part $\eta$. Instead of inverting the operator $(\omega+i \eta-H)$ one sets up the following equation for the state $|Y\rangle$

$$
\left(\left(E_{0}+\omega-H\right)^{2}+\eta^{2}\right)|Y\rangle=-\eta B|\Psi\rangle
$$

where $E_{0}$ is the ground state energy of the chain. From this one can calculate the spectral function by

$$
\rho(\omega)=-\frac{1}{\pi} \mathfrak{I m}\langle\Psi|A| Y\rangle
$$

One can now formulate this linear equation as a minimization problem [128] of the following functional

$$
W(\omega, \eta)=\left\langle\Phi\left|\left(E_{0}+\omega-H\right)^{2}+\eta^{2}\right| \Phi\right\rangle+2 \eta\langle\Phi|B| \Psi\rangle .
$$

The state $|\Phi\rangle$, which minimizes this functional equals the solution to the equation (3.14)

$$
\left|\Phi_{\text {min }}\right\rangle=|Y\rangle \text {. }
$$

With this approach it is possible to calculate the spectral function of the Anderson model by using the DMRG.

\subsubsection{Algorithm}

At the end of this section I will summarize the DMRG algorithm. First, one brings the Anderson impurity Hamiltonian into the discrete form of a linear chain. In contrast to the NRG it is possible to discretize the conduction band, for example, in linear intervals resulting in non-exponentially decreasing hopping parameters. By this one can control the resolution of the conduction band. As usual, the only interaction between spin-up and spin-down electrons occur on the impurity. Thus it is possible to build up the chain with all the upelectrons to the left, the impurity sites in the middle and the down-electrons to the right. This results in a small local Fock space consisting of only two states: no electron or one electron. This accelerates the whole procedure. One now uses the DMRG for finding the ground state $|\Psi\rangle$ of the whole chain. By increasing and decreasing the size of the outer combined blocks, called sweeping, one improves the basis set used for setting up the Hamiltonian and finally is able to calculate a very accurate ground state wave function. With $|\Psi\rangle$ it is possible to compute static expectation values, such as the occupation of the impurity. When calculating the spectral function one cannot simply use the basis set just gained for the ground state. This will give a very poor result for the spectral function as the basis set is optimized for the ground state and cannot describe the exited state $B|\Psi\rangle$ very well. For each frequency $\omega$, one has to calculate the exited state $B|\Psi\rangle$ and the state $|Y\rangle$ as defined in equation (3.14). One has to improve the basis set by sweeping for these two states and the real part of the spectral function $|X\rangle$ given by

$$
|X\rangle=\frac{H-E_{0}-\omega}{\eta}|Y\rangle .
$$


This means that one includes not only the ground state wave function into the reduced density matrix but also these three exited states [128]. The density matrix is then simply written as sum

$$
\rho_{i j}=\sum_{p} \alpha_{p} \sum_{k} \Psi_{i k}^{\alpha} \Psi_{j k}^{\alpha}
$$

where $p$ labels the four states and $\alpha_{p}$ is some amplitude for the importance of the state. In my calculations I usually used $\alpha=0.4$ for the ground state and $\alpha=0.2$ for each of the exited states. By sweeping for each frequency point one improves the basis set and is finally able to calculate the spectral function.

The coefficient $\eta$ results in a Lorentzian broadening of the spectral function. Decreasing $\eta$ results in a worse convergence to the solution of the equation for the correction-vector (3.14). Not only more time is needed for finding the solution, one also has to include more states $m$ in the density matrix for calculating a stable solution. 
CHAPTER 4

\section{NRG and DMRG Spectral Functions for the Impurity Model}

\subsection{Introduction}

This chapter deals with a comparison between the numerical renormalization group (NRG) and the density matrix renormalization group (DMRG) as impurity solvers. Both methods were introduced in chapter 3. The NRG is well established for calculating spectral functions for one- or two-orbital Anderson models $[94,105]$. These kind of calculations have been done for approximately 20 years. In contrast, the DMRG algorithm, which was used here for the computation of spectral functions, is rather young existing in this form for only 10 years [126-128]. Calculations for impurity systems have been performed for 5

years $[115,116]$. From this fact it is clear that one should test the DMRG as impurity solver before using it in the framework of the DMFT. Intensive tests were performed in the Ph.D. thesis of C. Raas [129].

As the DMRG spectral functions are Lorentz-convoluted, it is recommendable to look at the deconvolution of the data. Therefore, I will give a short introduction into the topic of deconvolution in this chapter. Finally, I will compare the spectral functions of the DMRG and the NRG for different interaction strengths, doping and magnetic fields. All three parameters are important, when using the DMRG in the framework of the dynamical mean field theory for calculating magnetic phase diagrams. Based on these results I will discuss the strengths and the weaknesses of the DMRG as an impurity solver. 


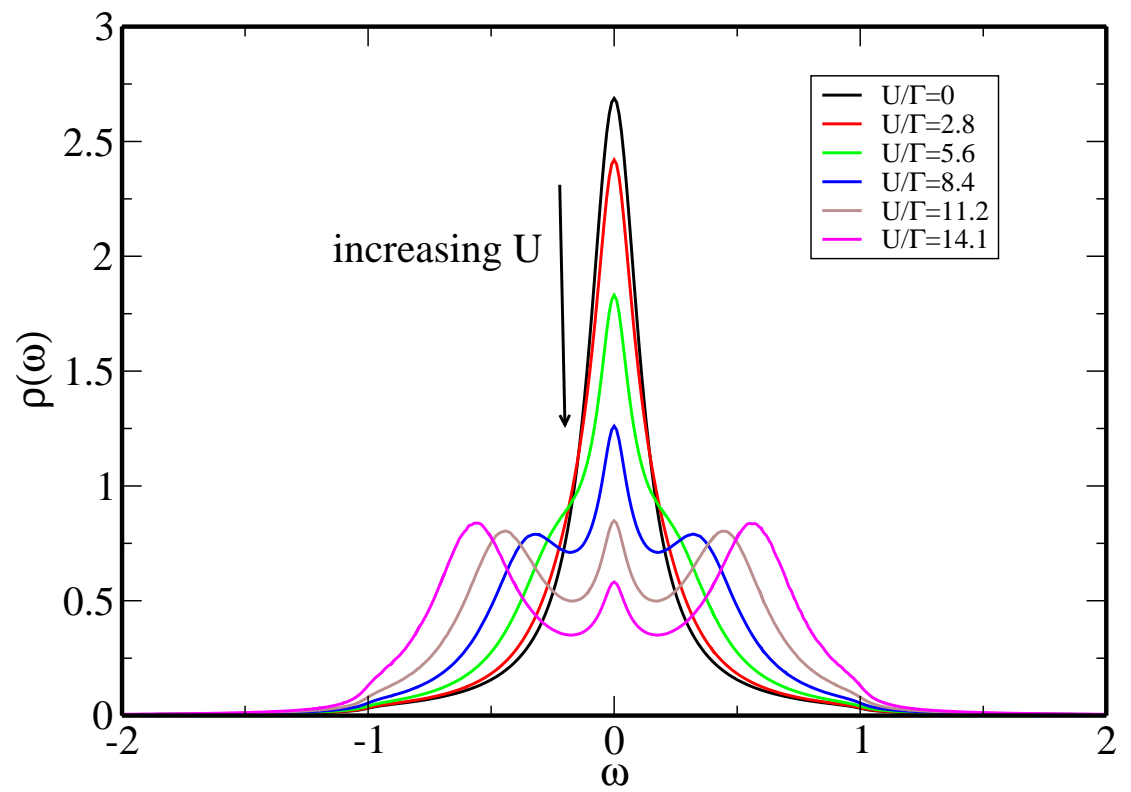

Figure 4.1: Spectral functions from DMRG calculations for the SIAM for $\Gamma=0.07$ and different interaction strengths. Friedel sum rule states $\rho(0)=\frac{1}{\pi \Gamma} \approx 4.54$ in this case. Within the DMRG a broadening of $\eta=0.05$ was used.

The single impurity Anderson model (SIAM), as introduced in chapter 3, reads

$$
\begin{array}{r}
H_{A n d}=\sum_{\sigma} \int_{-D}^{D} d \epsilon \epsilon c_{\epsilon, \sigma}^{\dagger} c_{\epsilon, \sigma}+\sum_{\sigma} \epsilon_{d} c_{d, \sigma}^{\dagger} c_{d, \sigma}+U c_{d, \uparrow}^{\dagger} c_{d, \uparrow} c_{d, \downarrow}^{\dagger} c_{d, \downarrow} \\
\sum_{\sigma} \int_{-D}^{D} d \epsilon V(\epsilon) \sqrt{\rho(\epsilon)}\left(c_{\epsilon, \sigma}^{\dagger} c_{d, \sigma}+c_{d, \sigma}^{\dagger} c_{\epsilon, \sigma}\right) .
\end{array}
$$

A constant coupling $V(\epsilon) \sqrt{\rho(\epsilon)}=$ const between the conduction band and the impurity is chosen throughout this chapter. The hybridization is defined as

$$
\Gamma=\pi V(\epsilon)^{2} \rho(\epsilon)=\pi V^{2} \rho \quad \text { for } \quad \epsilon \in[-D, D]
$$

Figure 4.1 shows spectral functions of the SIAM for different interaction strengths obtained by the DMRG. The general form of the spectral functions agrees very well with the known results [98]. One can see the resonance at the Fermi energy, $\omega=0$, called Kondo resonance. For large enough interaction strengths, additional high energy peaks, called Hubbard peaks, are visible. The spectral weight is transferred from the central peak to the Hubbard peaks when increasing the interaction. But already in this figure one should notice that the Friedel sum rule [98], which states that the half-filled SIAM spectral function is pinned at $\omega=0$ to $\rho(0)=\frac{1}{\pi \Gamma}$, is not fulfilled. I will discuss the spectral functions in more detail later in this chapter. 

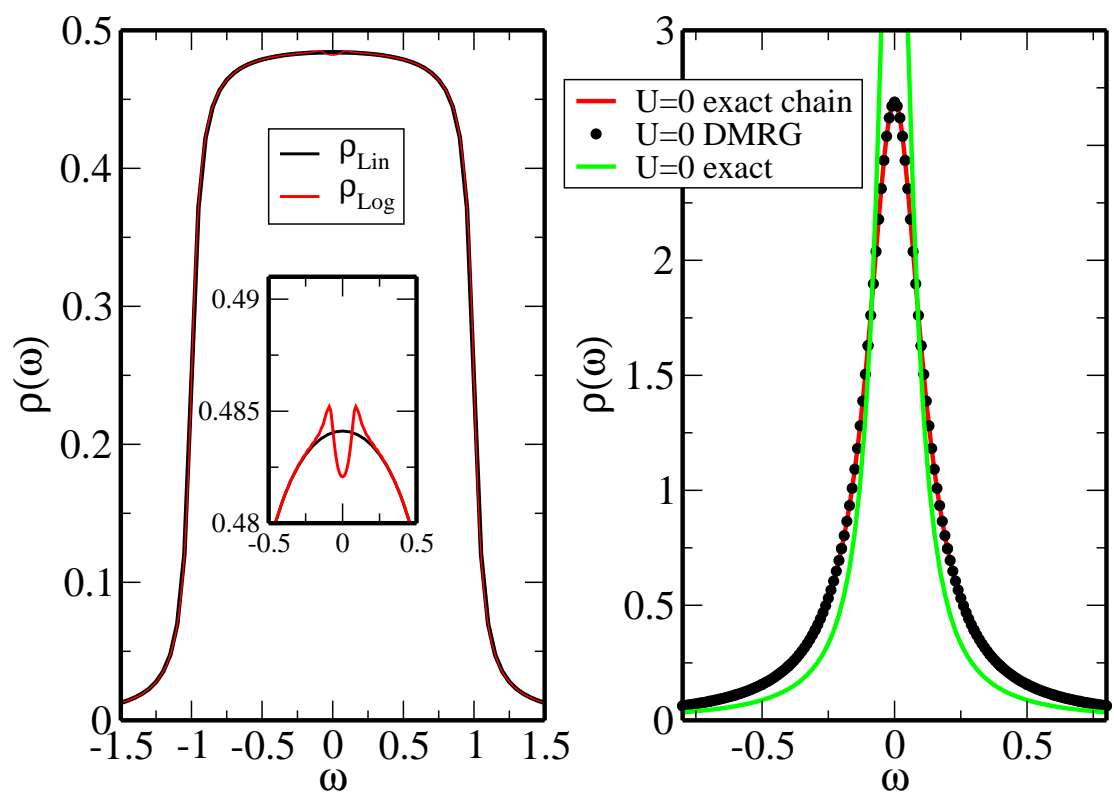

Figure 4.2: Left: Bath spectral function obtained by the diagonalization of the hopping chain. Two different discretization schemes were used. The black (red) line corresponds to linear (linear and logarithmic) intervals. The broadening $\eta=0.05$ was used. Right: $U=0, \Gamma=0.07$ spectral function of the SIAM. The black points and the red line are obtained by diagonalizing the hopping chain. The green curve represents the exact solution to the SIAM. For the DMRG calculations a discretization into 81 intervals was used.

\subsection{Used Discretization Schemes}

Before using either the NRG or the DMRG for the SIAM, one has to discretize the band states (chapter 3). The discretization for the DMRG is quite arbitrary. In figure 4.2 I show two resulting bath Green's functions and the non-interacting impurity spectral function. The bath spectral function represents the form of the bath, which couples to the impurity. The results throughout this chapter were done for a constant bath spectral function $\rho_{0}(\epsilon)=0.5$ for $\epsilon=[-1,1]$. I have tested two kinds of discretization schemes in the DMRG. The black line in the left panel of figure 4.2 corresponds to a pure linear discretization, while the red line corresponds to a linear discretization for large frequencies $|\omega|>0.1$ and a logarithmic discretization for $|\omega|<0.1$. The reason for the logarithmic part is to improve the resolution around the Fermi energy. The bath spectral functions were calculated by exact diagonalization of the chain by

$$
\rho(\omega+i \eta)=-\frac{1}{\pi} \mathfrak{I m}\left\langle\Psi_{0}\left|c \frac{1}{\omega+i \eta-H} c^{\dagger}\right| \Psi_{0}\right\rangle
$$

where $c^{\dagger}$ creates an electron on the last site of the bath sites, which directly couples to the impurity. I have used the same broadening $\eta$ in this calculation as in the DMRG calculations. One can see, that this approach quite nicely reproduces a nearly constant bath continuum near $\omega \approx 0$. The discrepancy between 
0.5 and 0.48 as well as the curvature at the band edges can be explained by the broadening. The logarithmic broadening shows slight oscillations around the point, where I changed from linear to logarithmic discretization. Throughout this chapter I used 81 intervals for the discretization of the bath continuum. The complete DMRG chain with splitting of the spin-up and the spin-down states to the left and right of the impurity adds up to 164 sites. The right panel in figure 4.2 corresponds to the non-interacting SIAM, $U=0$. The red line and the black points, which lie exactly on the red line, are the calculated and broadened spectral function in discretized form. "Exact" means that the hopping chain was diagonalized without DMRG. The green curve corresponds to the exact spectral function of the SIAM. The DMRG reproduces the exact spectral function, which can be obtained after the discretization of the bath states. The difference between the exact curve and the discretized spectral function is due to the discretization and the broadening.

\subsection{Deconvolution}

Due to numerical reasons and finite size effects, see chapter 3 , it is not possible to use equation 4.1 for arbitrary small broadening $\eta$ within the DMRG. From

$$
\begin{aligned}
G(z) & =\left\langle\Psi_{0}\left|c \frac{1}{z-H} c^{\dagger}\right| \Psi_{0}\right\rangle \\
& =\int \frac{\rho(\omega)}{z-\omega} d \omega \\
\Rightarrow \rho(\omega+i \eta) & =-\frac{1}{\pi} \mathfrak{I m} \int \frac{\rho\left(\omega^{\prime}\right)}{\omega+i \eta-\omega^{\prime}} d \omega^{\prime} \\
& =\frac{\eta}{\pi} \int \frac{\rho\left(\omega^{\prime}\right)}{\left(\omega-\omega^{\prime}\right)^{2}+\eta^{2}} d \omega^{\prime}
\end{aligned}
$$

one can see, that the spectral function calculated within the DMRG is convoluted with a Lorentzian. Deconvolution is an ill-posed problem due to two reasons. The first reason is a physical one: The unbroadened spectral function of a finite system, like the finite chain used within DMRG, consists of a finite number of delta-peaks. Of course, this number is rather big. Nevertheless, finite size effects will most likely be visible. As one is interested in the spectral function, when there is a continuum of bath states, these finite size effects are undesirable. Therefore, it is actually not the goal to do a complete deconvolution. But even if a complete deconvolution would be the goal, it would be numerically impossible. The mathematical reason for this is the sensitivity on the input data points. Only small changes in $\rho(\omega+i \eta)$ can lead to rather big changes in $\rho(\omega)$. As the inversion of the integral is done numerically, errors will occur. Another way to formulate the last statement is, that within a given error for the inversion there is more than one solution.

\subsubsection{Matrix Inversion}

A straight forward idea for the deconvolution is to consider the spectral function as a discrete set of points. Thus the integral in equation (4.2) can be written as 
a matrix operation. Let $\rho_{i}=\rho\left(\omega_{i}\right)$ with $\omega_{i+1}-\omega_{i}=d \epsilon$, one can approximate equation (4.2) as

$$
\rho\left(\omega_{i}+i \eta\right)=\frac{\eta}{\pi} \sum_{j} \frac{\rho_{j}}{\left(\omega_{i}-\omega_{j}\right)^{2}+\eta^{2}} d \epsilon=: L_{i j} \rho_{j},
$$

defining the matrix of the Lorentz-convolution $L_{i j}$. This matrix can in principle be inverted. Thus the desired spectral function can be calculated by

$$
\rho_{j}=L_{i j}^{-1} \rho\left(\omega_{i}+i \eta\right)
$$

If one uses a fine mesh in frequency space, the matrix $L_{i j}$ will have eigenvalues, which are approximately zero thus making an inversion practically impossible. Pseudo-inverse techniques can be applied yielding results for the spectral function. These deconvoluted spectral functions have commonly two problems: They are not positive definite and they show strong oscillations. Thus better methods should be used for deconvolution [129].

\subsubsection{Maximum Entropy Ansatz}

A method, which is frequently used for analytic continuation of Quantum Monte Carlo (QMC) data or image restoration, for example, in astronomy, is Maximum Entropy [130-132]. This kind of analytic continuation is closely related to deconvolution as both methods try to invert the same integral. But in the analytic continuation of QMC data one tries to calculate $\rho(\omega)$ from $G(i \omega)$.

The Shannon information entropy is written as

$$
S[\rho(\omega)]=-\int d \omega \rho(\omega) \log \frac{\rho(\omega)}{D(\omega)},
$$

in which $D(\omega)$ is the default model. The entropy without any further conditions is maximal for $\rho(\omega)=D(\omega)$. Throughout this thesis I use a constant default model $D(\omega)=1$. The same default model was used in the deconvolution of DMRG data in the work of C. Raas [129] and P. Dargel [133]. The reason for using a constant default model is the wish for a least biased deconvolution strategy. Finding the maximum is done under the condition that $\rho(\omega)$ is consistent with the $N$ calculated data points $\rho\left(\omega_{i}+i \eta\right)$, which are supposed to be deconvoluted, meaning

$$
\rho\left(\omega_{i}+i \eta\right)=\frac{\eta}{\pi} \int \frac{\rho(\omega)}{\left(\omega-\omega_{i}\right)^{2}+\eta^{2}} d \omega
$$

Using Lagrange-multipliers $\lambda_{i}$ to enforce this condition, one can find the extremum of the entropy by

$$
\begin{aligned}
0 & =\frac{\delta S[\rho(\omega)]}{\delta \rho(\omega)} \\
& =\int d \omega\left(-\log \rho(\omega)-1+\sum_{i} \lambda_{i} \frac{\eta}{\pi} \frac{1}{\left(\omega-\omega_{i}\right)^{2}+\eta^{2}}\right)
\end{aligned}
$$


giving an ansatz for the spectral function as

$$
\rho(\omega)=\exp \left(\sum_{i} \lambda_{i} \frac{\eta}{\pi} \frac{1}{\left(\omega-\omega_{i}\right)^{2}+\eta^{2}}\right) .
$$

This ansatz includes $N$ free parameters $\lambda_{i}$, which can be calculated by equation (4.3). Thus one has to solve $N$ non-linear coupled equations yielding the parameters $\lambda_{i}$. For the results shown in this chapter the system of equations was solved by steepest descent until the desired precision of about

$$
\frac{1}{N} \chi^{2}[\rho(\omega)]:=\frac{1}{N} \sum_{i}\left(\rho\left(\omega_{i}+i \eta\right)-\frac{\eta}{\pi} \int \frac{\rho(\omega)}{\left(\omega-\omega_{i}\right)^{2}+\eta^{2}} d \omega\right)^{2}<0.0002
$$

was gained.

The algorithm can be improved by additional assumptions. For example, one can enforce the norm $\int d \omega \rho(\omega)=1$ being always fulfilled. Another point is to assume error afflicted data. Then one can include the variance

$$
\chi^{2}[\rho(\omega)]=\sum_{i}\left(\rho\left(\omega_{i}+i \eta\right)-\frac{\eta}{\pi} \int \frac{\rho(\omega)}{\left(\omega-\omega_{i}\right)^{2}+\eta^{2}} d \omega\right)^{2}
$$

into the functional, which is maximized, yielding

$$
Q=S[\rho(\omega)]-A \chi^{2}[\rho(\omega)]
$$

The constant $A$ can then be used for tuning the resulting $\rho(\omega)$, so that $\chi^{2}$ equals the magnitude of the assumed errors.

Figure 4.3 shows an example for a deconvolution. I assume no errors in the pure DMRG data. The black line corresponds to the DMRG output for a SIAM with $\Gamma=0.07$ and $U=0.8$. The blue line represents the NRG spectral function. One should notice two things: The Kondo-peak is strongly smeared out in the DMRG-data. On the other hand, the Hubbard satellites are much better resolved within the DMRG than in the NRG. Using now deconvolution on the DMRG data, all peaks become stronger. Nevertheless the Kondo peak having a width of $T_{K} \approx 0.008$ cannot be resolved. Moreover, in the deconvoluted spectral function additional oscillations occur, as can be seen at $\omega \approx \pm 0.15$. These oscillations are unphysical and due to the deconvolution. Similar oscillations occur in much stronger form in the matrix based deconvolution. It is usually very difficult to get rid of these artifacts. Therefore, it is very important to have knowledge about this fact, so that one can interpret this oscillations properly.

\subsection{Comparison between DMRG and NRG}

\subsubsection{Single Impurity Anderson Model}

I will now present several results which show spectral functions for the SIAM. In all figures I have used $\Gamma=0.07$. The initial DMRG broadening was always $\eta=0.05$. Figure 4.4 shows the spectral function for different ratios between the 


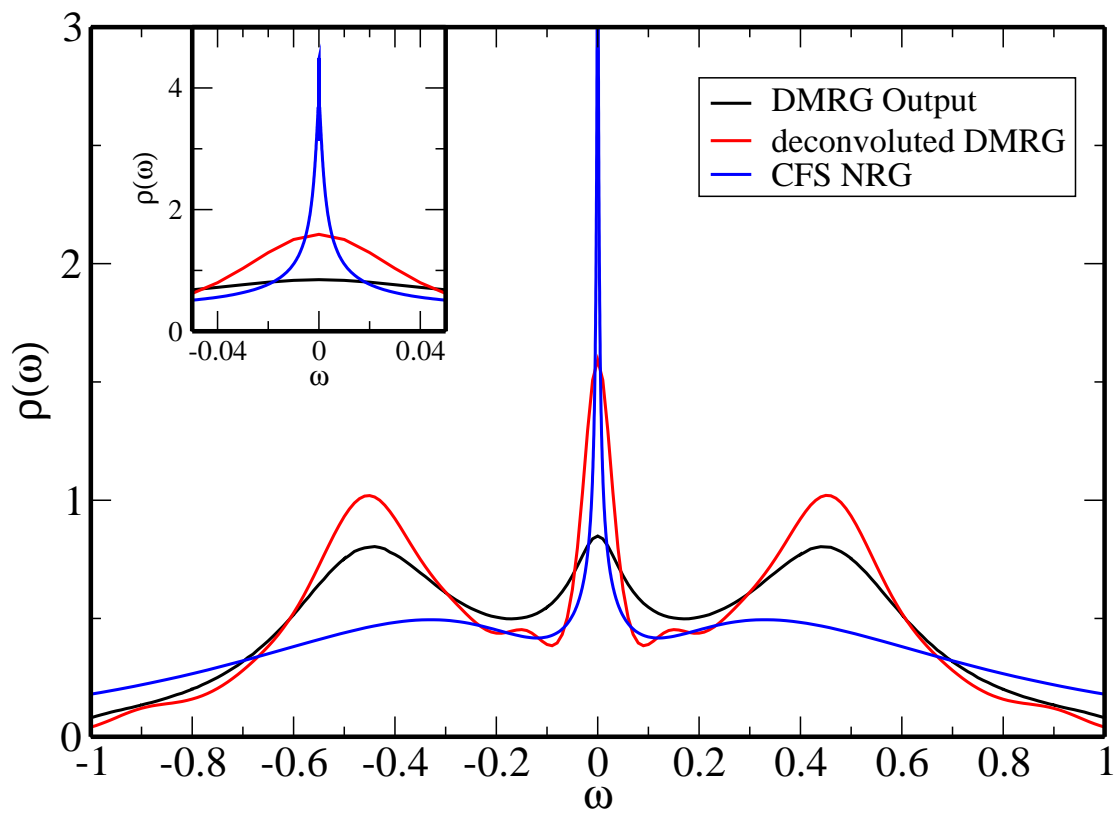

Figure 4.3: Spectral functions for $U=0.8$ and $\Gamma=0.07$. One can see the pure DMRG output and the comparison between the complete Fock space (CFS) NRG and the deconvoluted DMRG spectral function. The initial DMRG broadening was $\eta=0.05$.

interaction strength and the hybridization $\Gamma$. Continuous lines correspond to deconvoluted DMRG results and the dashed lines correspond to NRG results. The lower panel in figure 4.4 shows a magnification of the frequencies around the Fermi energy $\omega=0$. Two things should be obvious: The DMRG results resolve the Hubbard bands at large frequencies very well. But the Kondo resonance at $\omega=0$ is resolved very badly. For the NRG results the opposite statement is true. Due to the logarithmic broadening in the NRG, the Hubbard bands are completely smeared out. Both facts hold true for all figures shown in this chapter. Quite recently it was possible to increase the resolution of the NRG by a special kind of z-averaging [134]. Figure 4.5 shows the spectral functions for $U / \Gamma=11.2$. There are two DMRG calculations included corresponding to a linear discretization and a logarithmic discretization. The height of the Kondo peak within the logarithmic discretization is reduced, thus failing to improve the resolution of the low energy features. This is not astonishing, as the height is mainly limited by the broadening $\eta$. Additionally, I have included in figure 4.5 the NRG spectral functions using the ordinary NRG broadening (CFS NRG) and the high resolution broadening (HR NRG). One can see how the high resolution NRG can resolve the Kondo peak as well as the Hubbard bands with very high accuracy.

For calculating magnetic phase diagrams within the DMFT using the DMRG as an impurity solver, it is necessary that the DMRG is able to calculate spectral functions away from half-filling and is able to handle magnetic fields. Figures 4.6 and 4.7 show that both cases can be treated using the DMRG. Figure 4.6 shows the comparison between the DMRG and the NRG for different on-site 

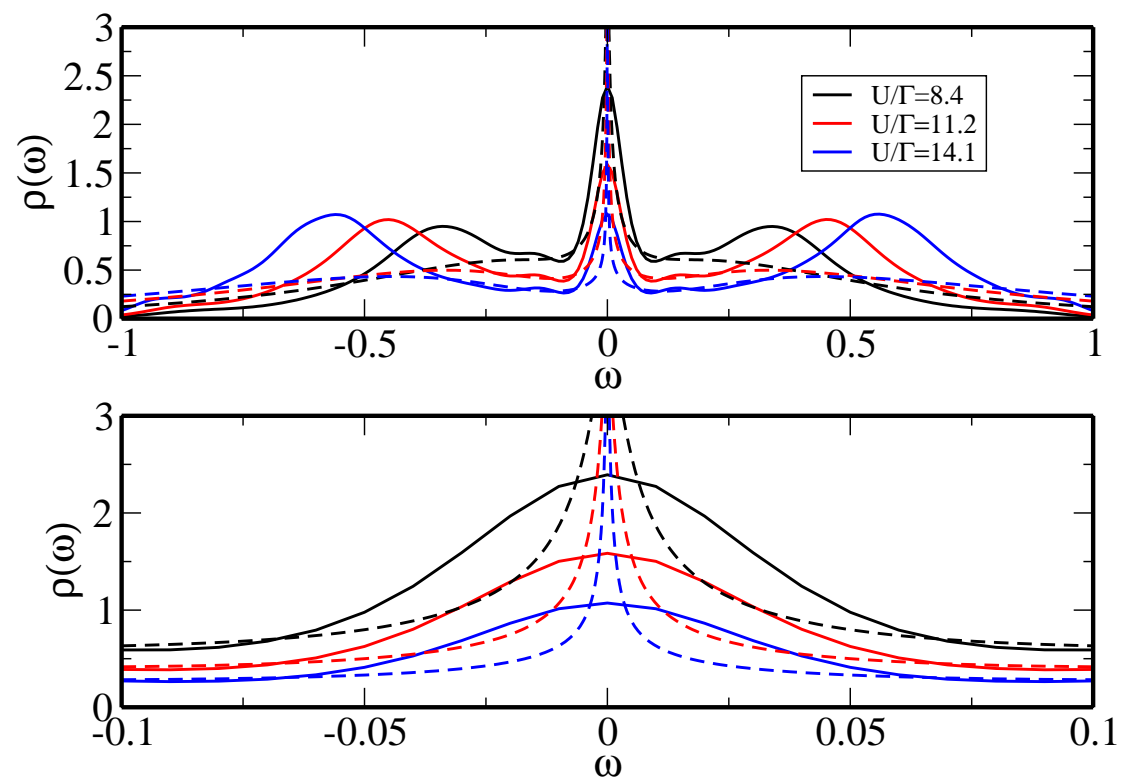

Figure 4.4: Spectral functions for $\Gamma=0.07$ and different interaction strengths. Continues lines correspond to the DMRG, dashed lines to the NRG. The lower panel shows a magnification of the upper panel around the Fermi energy $\omega=0$.

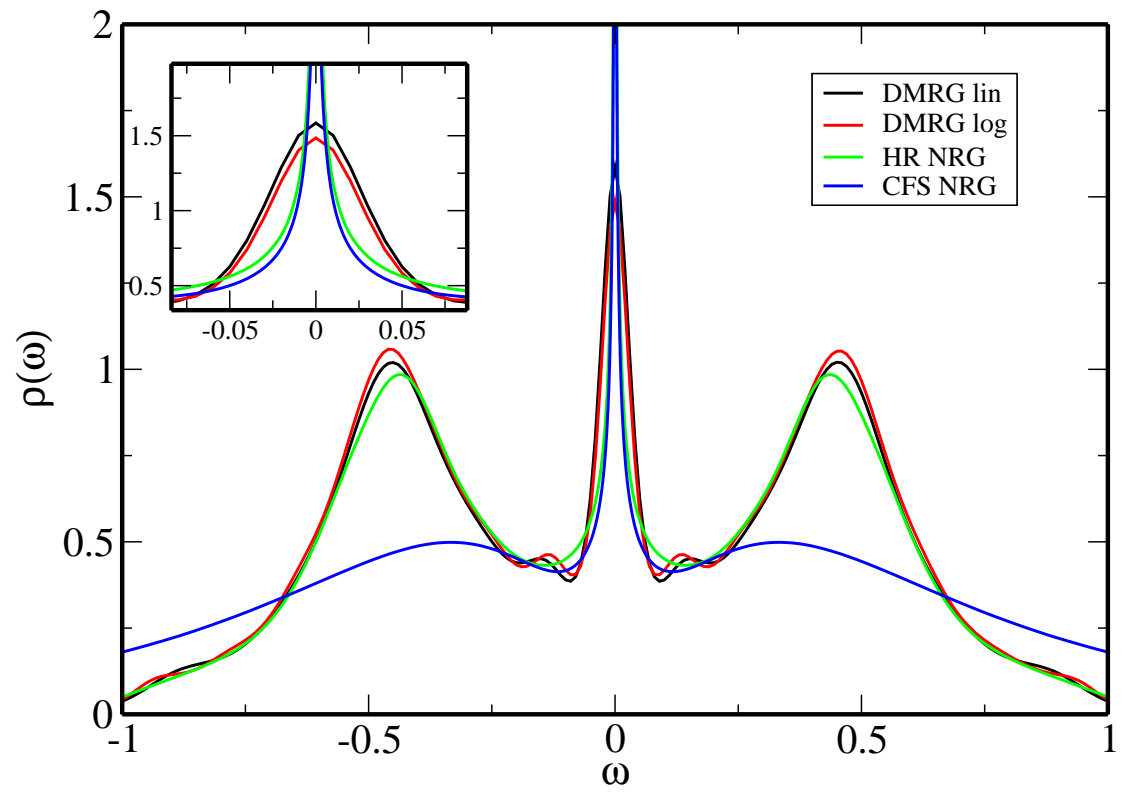

Figure 4.5: Spectral functions for $\Gamma=0.07$ for $U / \Gamma=11.2$. The black and the red line correspond to deconvoluted DMRG results with either linear discretization or logarithmic discretization of the conduction band. The green line represents the high resolution NRG explained in the text (This data was provided by Rok Zitko). The blue line corresponds to the complete Fock space NRG. The inset shows a magnification of the region around the Fermi energy. 


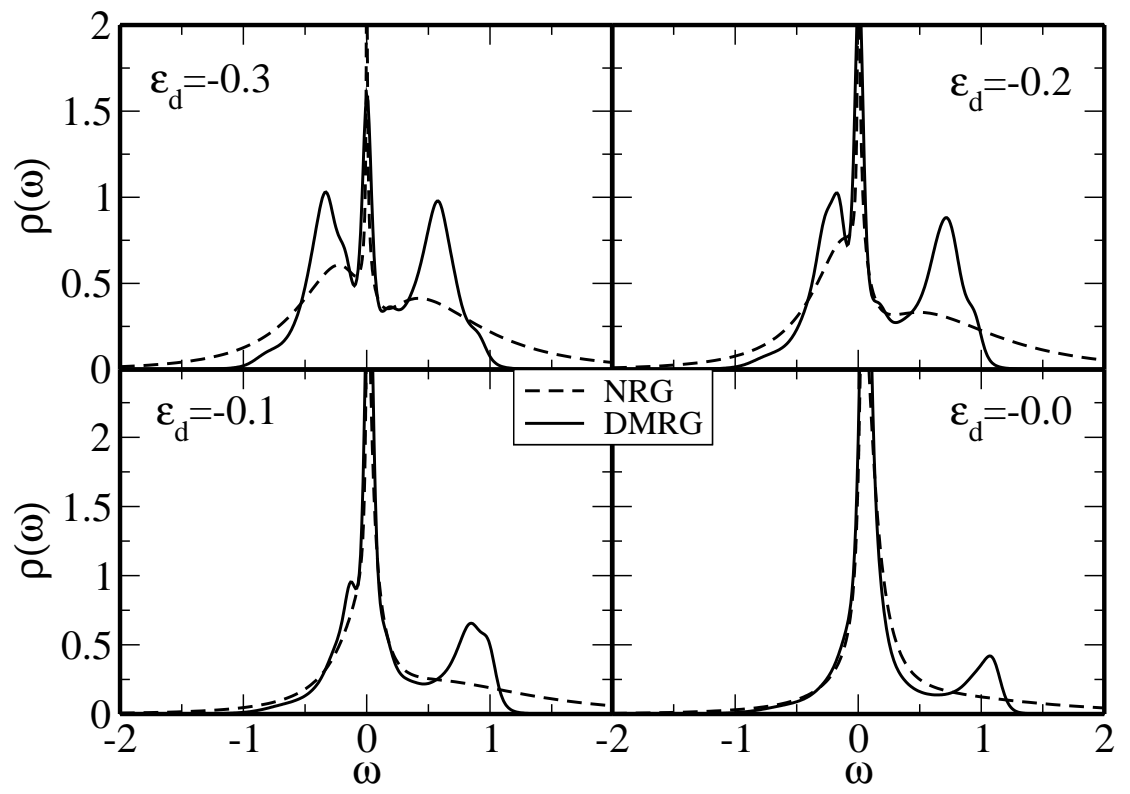

Figure 4.6: Spectral functions for the SIAM with $\Gamma=0.7, U=0.8, T_{k} \approx 0.008$, and different on-site energies.

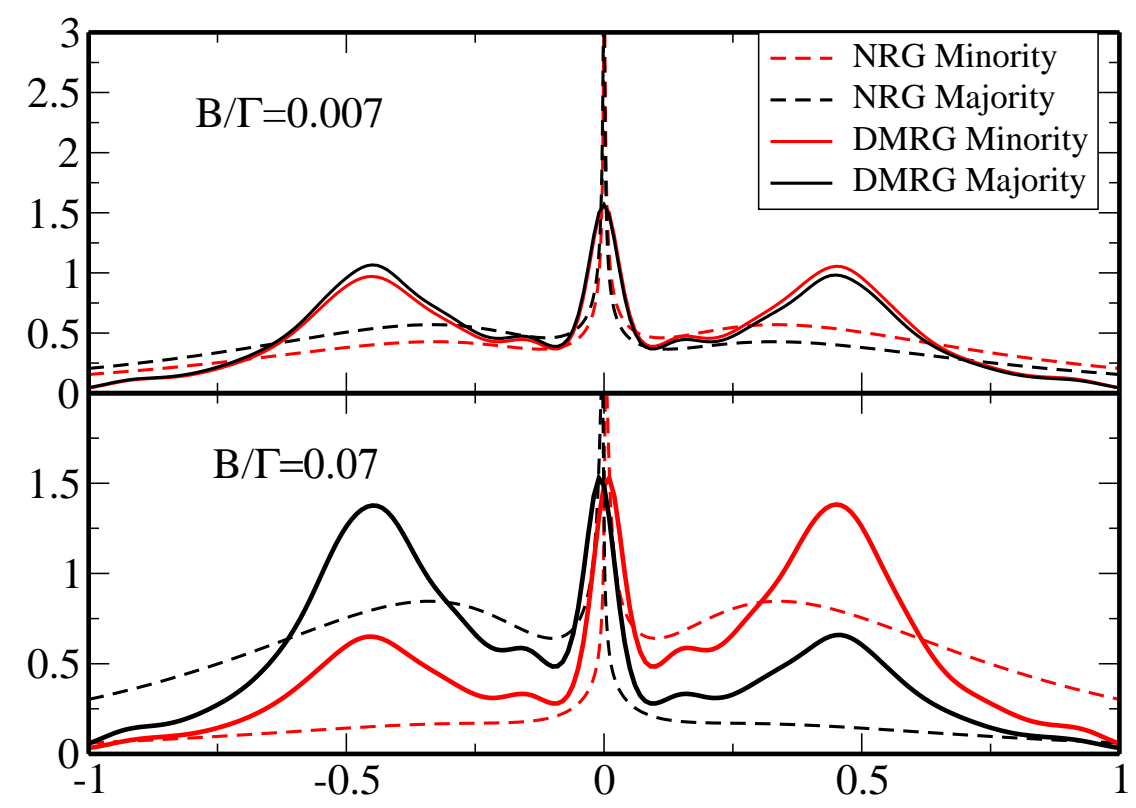

Figure 4.7: Spectral functions for the SIAM with $\Gamma=0.7, U=0.8, T_{k} \approx 0.008$, and different magnetic fields. 
energies, resulting in different occupations of the impurity. Within the NRG results one can see, how the spectral weight is shifted to the right, but one cannot see any substructures in the Hubbard bands. In contrast, within the DMRG results one can nicely see, how the left Hubbard band moves into the Kondo resonance.

Figure 4.7 depicts the results for two different strengths of the magnetic field. In the beginning of the NRG, there was the problem that the NRG dramatically underestimated the effect of small magnetic fields. This problem was firstly solved by introducing the reduced density matrix [111]. The reduced density matrix ensures the correct influence of the ground state on the high energy features in the spectral function. As in the DMRG the correct ground state is used for each frequency, such problems are supposed to be absent. This statement is proven in figure 4.7. The splitting of the majority and minority spin spectral functions is comparable in strength for both methods. Thus the DMRG is able to handle small and large magnetic fields acting on the impurity.

\subsubsection{Two-Orbital Anderson Model}

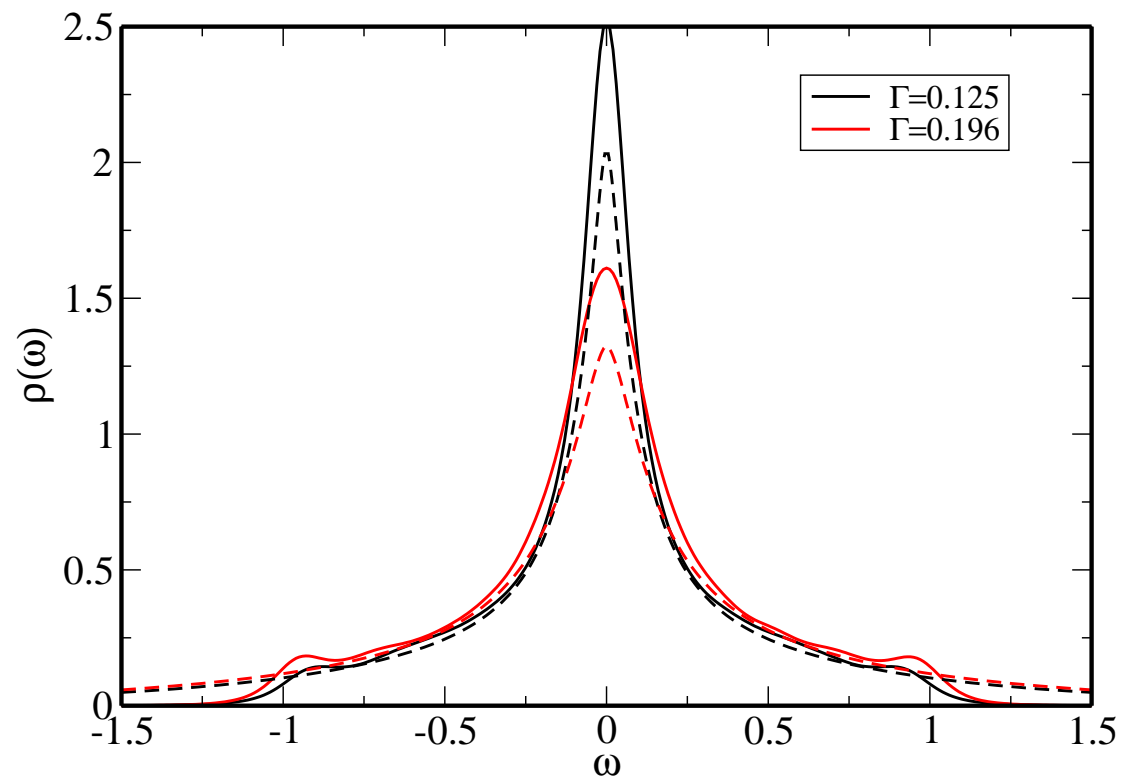

Figure 4.8: Two-orbital Anderson model for $U=0.4, J=0, U^{\prime}=0.4$, and two different hybridizations $\Gamma$. Continuous lines are DMRG results, dashed lines NRG results.

Finally, it is an interesting question to see, if the DMRG can handle the multiorbital Anderson model. An intensive study for a simplified two impurity Anderson model was done by S. Nishimoto et al. [135]. Figure 4.8 shows the results for the two-orbital Anderson model. The construction of the one-dimensional chain was such, that the Fock space states of one orbital were to the left of the impurity and the states of the other orbital to the right. Figure 4.8 and 4.9 shows that it is possible to do multi-orbital impurity calculations using the DMRG. Interestingly, the deconvoluted DMRG spectral functions in figure 4.8 

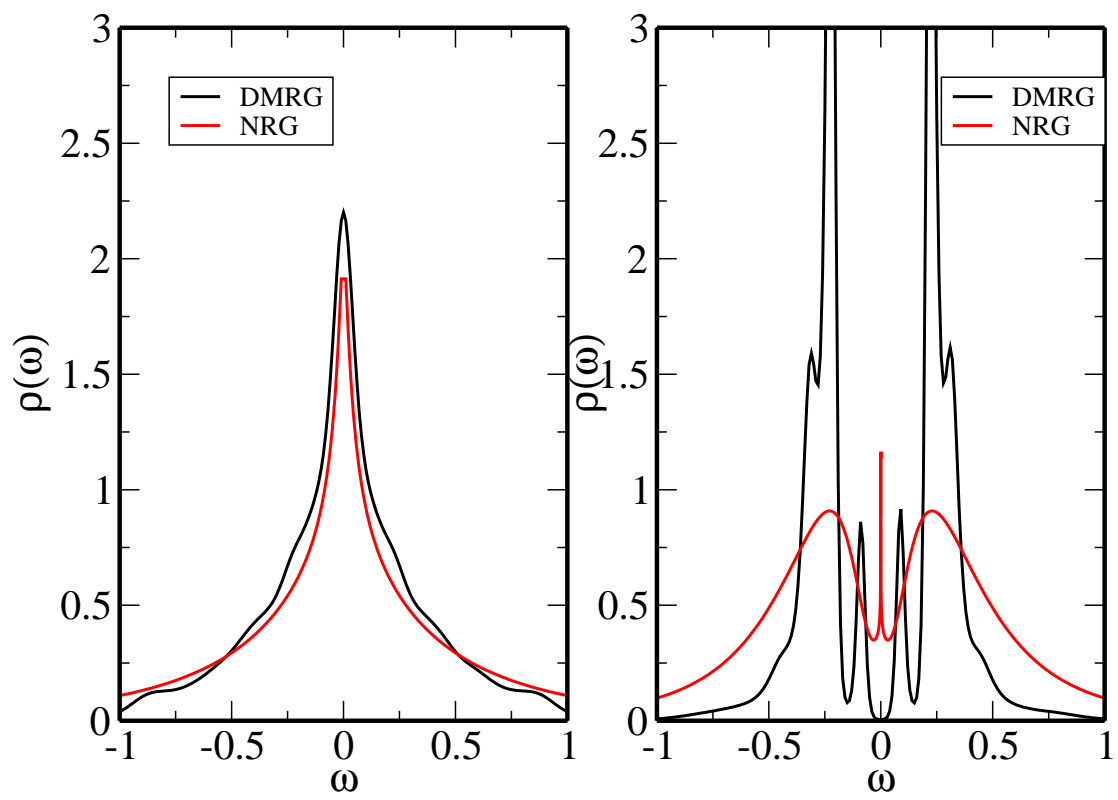

Figure 4.9: Two-orbital Anderson model for $U=0.4, J=0.1, U^{\prime}=0.2$, and two different hybridizations $\Gamma=0.125$ (left) and $\Gamma=0.031$ (right).

with $J=0$ have more spectral weight at the Fermi energy $\omega=0$ than the NRG. This is in contrast to the calculations in the one-orbital Anderson model. As the Friedel sum rule is supposed to hold in this case, one can see, that the DMRG fulfills the sum rule more accurately. For $\Gamma=0.125$ the sum rule predicts $\rho(0)=2.54$, and for $\Gamma=0.196$ it predicts $\rho(0)=1.62$. Two-orbital calculations using the NRG have the big problem, that the Fock space must be built up from 16 states at the same time. Although the shown NRG results were done with $N_{k}=5000$ states kept after the truncation, this number corresponds to $N_{K}=70$ states in the one-orbital model. This explains the discrepancies of the NRG results to the Friedel sum rule. Another aspect in the spectral functions are the band edges at $\omega= \pm 1$. They are again completely smeared out in the NRG due to the large broadening at large frequencies. The DMRG spectral function nicely resolve these band edges. Finally, figure 4.9 shows results for $J=0.1$. Finite Hund's coupling has a profound consequence on the spectral function. The Kondo temperature can be considerably reduced by it [52]. The left panel in figure 4.9 shows the spectral function for relatively large hybridization $\Gamma$, for which no Hubbard peaks are present. In this situation the NRG and the DMRG are very similar. In the right panel one can see the spectral functions for a small hybridization $\Gamma$. The Kondo temperature is very small. It can still be resolved by the NRG, but the DMRG fails. In the DMRG spectral function one can see additional substructures in the Hubbard bands. But it is very difficult to say, if these structures are real or effects of the deconvolution. One should perform additional calculations with other conduction band discretization to check this result. 


\subsection{Summary}

In this chapter I compared results for the spectral functions of the NRG and the DMRG. As the NRG is well established for calculating spectral functions for arbitrary interaction strengths, impurity occupation, and magnetic fields, I mainly tested the DMRG algorithm in this chapter. I have shown that the DMRG is able to calculate dynamical properties of a SIAM reliably. The main problem for the DMRG is the broadening, which can be comparable to the width of the Kondo resonance, thus eventually failing to correctly resolve the low energy features. On the other hand, the DMRG resolves the high energy features like the Hubbard bands very well, at least better than within the NRG. Of course, one should mention that also in the NRG there are ways to improve the resolution of the Hubbard bands [134]. A way to improve the resolution within the DMRG is deconvolution. Although possible, there are limits to what extend structures can be resolved. As the focus of this thesis lies on magnetic phases, which are mainly stabilized due to the high energy features, there is the hope, that the DMRG can deal with these situations.

One very big advantage of the DMRG to the NRG could be seen in the twoorbital Anderson model. As the discretization within the DMRG and also the construction of the chain is rather arbitrary, one has the possibility to split the local Fock space states in the DMRG. This leads to a reduction of the number of states during one iterative diagonalization. In the DMRG, it is possible to separate the band states for each spin and orbital direction, as they are only interacting with each other directly on the impurity. This enables one to perform calculations for even more than two orbitals, as the chain size increases only linearly with the number of orbitals. 
CHAPTER 5

\section{One-orbital Hubbard model}

\subsection{Introduction}

In this chapter I will present my results for the situation, when there is effectively only one orbital left at the Fermi level. This can occur in the case of further symmetry reduction from cubic perovskite to tetragonal structure [14], see chapter 1.2. Assuming that there is no Hund's coupling between the partially filled orbital and the remaining localized electrons, or the remaining bands are completely filled or empty like in the case of cuprates [14], this situation can be modeled by the one-orbital Hubbard model (chapter 1.3)

$$
H=\sum_{i j, \sigma} t_{i j} c_{i, \sigma}^{\dagger} c_{j, \sigma}-\mu \sum_{i, \sigma} n_{i, \sigma}+U \sum_{i} n_{i, \uparrow} n_{i, \downarrow}
$$

where $t_{i j}$ describes the hopping of the electrons, $\mu$ is the chemical potential, and $U$ the amplitude for the electron-electron interaction. The model was already introduced in the first chapter. In this chapter I will examine the effects of all three parameters: Changing the form of the hopping, the ratio between hopping and interaction strength, and the occupation of the system by tuning the chemical potential $\mu$.

All calculations in this chapter were performed within DMFT for a Bethe lattice. In the second section I will address the Hubbard model with nearest neighbor (NN) hopping only, resulting in a semi-elliptic DOS. In the third section I compare DMRG with NRG results for this model. Finally, in the last section of this chapter I will introduce an additional next nearest neighbor (NNN) hopping $t_{2} \neq 0$ leading to an asymmetric DOS. As shown in chapter 2.5, the semi-elliptic density of states for NN-hopping becomes asymmetric and finally develops a singularity at the lower band edge for $t_{2}>0$ and $t_{2} / t_{1}>1 / 4$. For $t_{2}<0$ the DOS is reflected at $\omega=0$ compared to $t_{2}>0$. All the results remain 

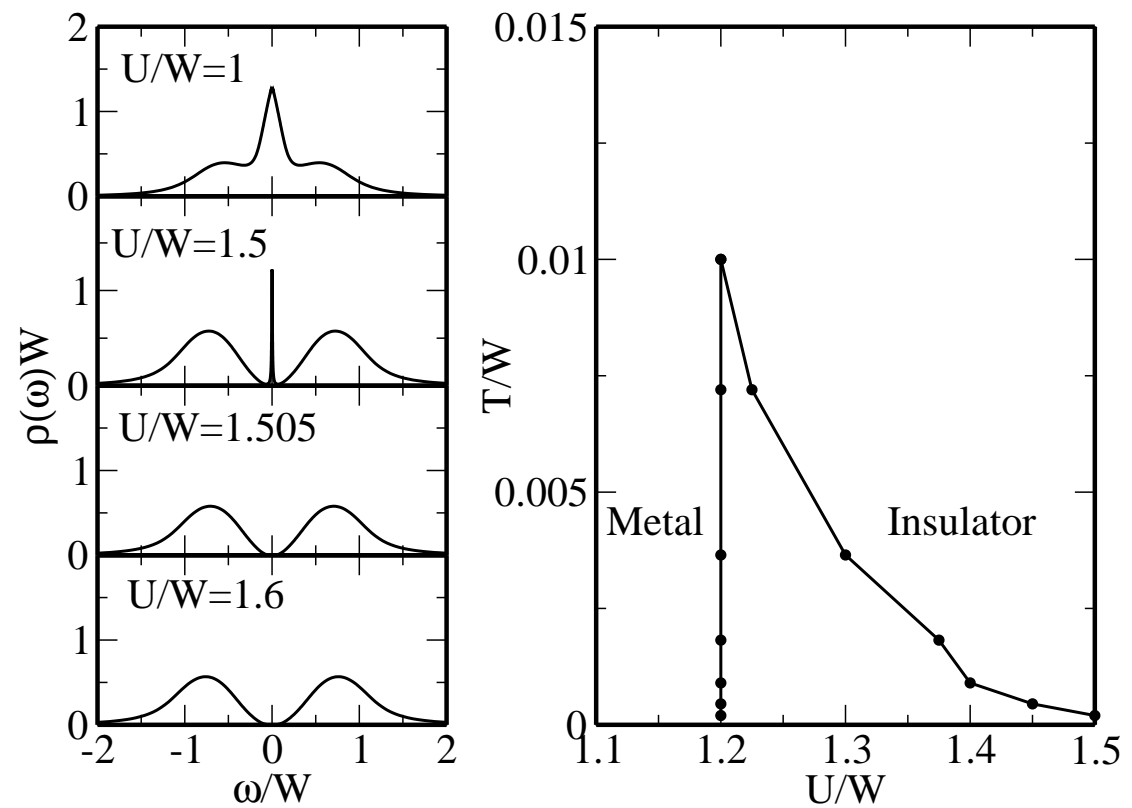

Figure 5.1: Metal insulator transition within DMFT for a Bethe lattice with semielliptic DOS. The left side of the figure shows the evolution of the spectral function $\rho(\omega)$ with increasing interaction strengths $U / W=(1,1.5,1.505,1.6)$ for $T=0$. The right side shows the first order transition lines from a metal to an insulator (right line) and from an insulator to a metal (left line). At $T / W \approx 0.01$ there is a critical point, above which there is only a cross-over between metallic and insulating solutions.

valid with occupation $n=1-x \longrightarrow n=1+x$ for $t_{2} \longrightarrow-t_{2}$. Therefore I will assume through out this chapter $t_{2} \geq 0$.

Most of the results in this chapter are published in Physical Review B [136] and New Journal of Physics [137].

\subsection{Semi-elliptic Density of States}

\subsubsection{Metal Insulator Transition}

Assuming that there is only NN-hopping $t$, the Bethe lattice has a symmetric semi-elliptic DOS, see chapter 2.5. The bandwidth $W=4 t$ of the noninteracting system is in the following used as the energy unit. The calculations are performed with DMFT/NRG for a half-filled system $n=1$ using the NRG discretization parameter $\Lambda=2$, and $N_{K}=1000$ kept states (see chapter 3). As I will show now, the paramagnetic metal insulator transition (PMIT) is strongly connected to the magnetic phase diagram of the Hubbard model. The PMIT is a numerically well analyzed phenomenon within DMFT [106,138-141]. The results obtained in my own calculations can be seen in figure 5.1 and are summarized as follows: When approaching the PMIT from the metallic phase a three peak structure develops with two Hubbard bands and a quasi-particle peak at the Fermi-energy $\omega=0[106,138]$. The width of the quasi-particle peak 
decreases for increasing interaction strengths. Finally at a critical interaction strength the quasi-particle peak vanishes. Thus an insulator is formed. The PMIT occurs for an interaction strength of about $U / W \approx 1.5$ at $T=0$. The left panel of figure 5.1 shows the evolution of the spectral function for increasing interaction strength $U$ for $T / W=2 \cdot 10^{-4}$. When starting the DMFT selfconsistency with an insulating medium, obtained from calculations with large interaction strength, the transition occurs at a lower critical value of the interaction, at which the gap vanishes. The right side of figure 5.1 shows these PMIT transition lines. The right line shows the transition starting with a metallic self-energy, while the left line corresponds to the transition when starting with an insulating self-energy. Between both lines there is a hysteresis region, where both insulating and metallic solutions can be stabilized. Therefore, the transition is of first order for temperatures below the critical temperature. Above the critical temperature there is a smooth cross-over between the metallic and the insulating phase. As I did not perform any $\Lambda \rightarrow 1$ scaling in the NRG, the transition lines and the critical endpoint do not exactly lie upon the DMFT/NRG results by R. Bulla et al. [106,138], but they are very close to them.

\subsubsection{Magnetic Phase Diagram}

The Hubbard model at half-filling exhibits super exchange, as described in chapter 1.4. Therefore it is not surprising to find a strong tendency towards antiferromagnetism. The antiferromagnetic phase in the Hubbard model at half-filling is a well analyzed fact, too [142-145]. It can be shown by perturbation theory that for an unfrustrated lattice, $t_{2}=0$, an antiferromagnetic phase exists at half-filling and $T=0$ for any finite interaction strength $U>0[142,143]$. The paramagnetic metal insulator transition, shown in figure 5.1, has a smaller critical temperature than the Néel-temperature of the antiferromagnetic phase $[70,140,146,147]$. This means that the PMIT is completely covered by the antiferromagnetic phase at half-filling. For analyzing the PMIT in this model the antiferromagnetic phase has to be suppressed artificially during the DMFT self-consistency cycle by, for example, averaging the spin components of the effective medium. A phase diagram, as seen in $\mathrm{V}_{2} \mathrm{O}_{3}$ (chapter 1.2.1), cannot be seen in the Hubbard model with semi-elliptic DOS. It has been argued that frustration might be able to suppress the antiferromagnetic phase revealing the PMIT $[70,147,148]$. I will come back to this point later in this chapter.

Besides antiferromagnetism, Nagaoka ferromagnetism was found in the Hubbard model $[144,149-151]$ for a simple cubic lattice. Interestingly, it is impossible to stabilize this kind of ferromagnetic phase for a Bethe lattice. In the rigorous derivation of Nagaoka for this ferromagnetic state [149], closed loops in the lattice structure are essential. As the Bethe lattice does not possess any closed loops this absence of Nagaoka ferromagnetism is in complete agreement with the theory. The interesting fact is, that the lattice structure only enters the DMFT calculation via the DOS. So changing the DOS from Gaussian with exponentially "long tails" for the hyper-cubic lattice, where Nagaoka ferromagnetism has been observed, to the semi-elliptic DOS with finite support, averts 

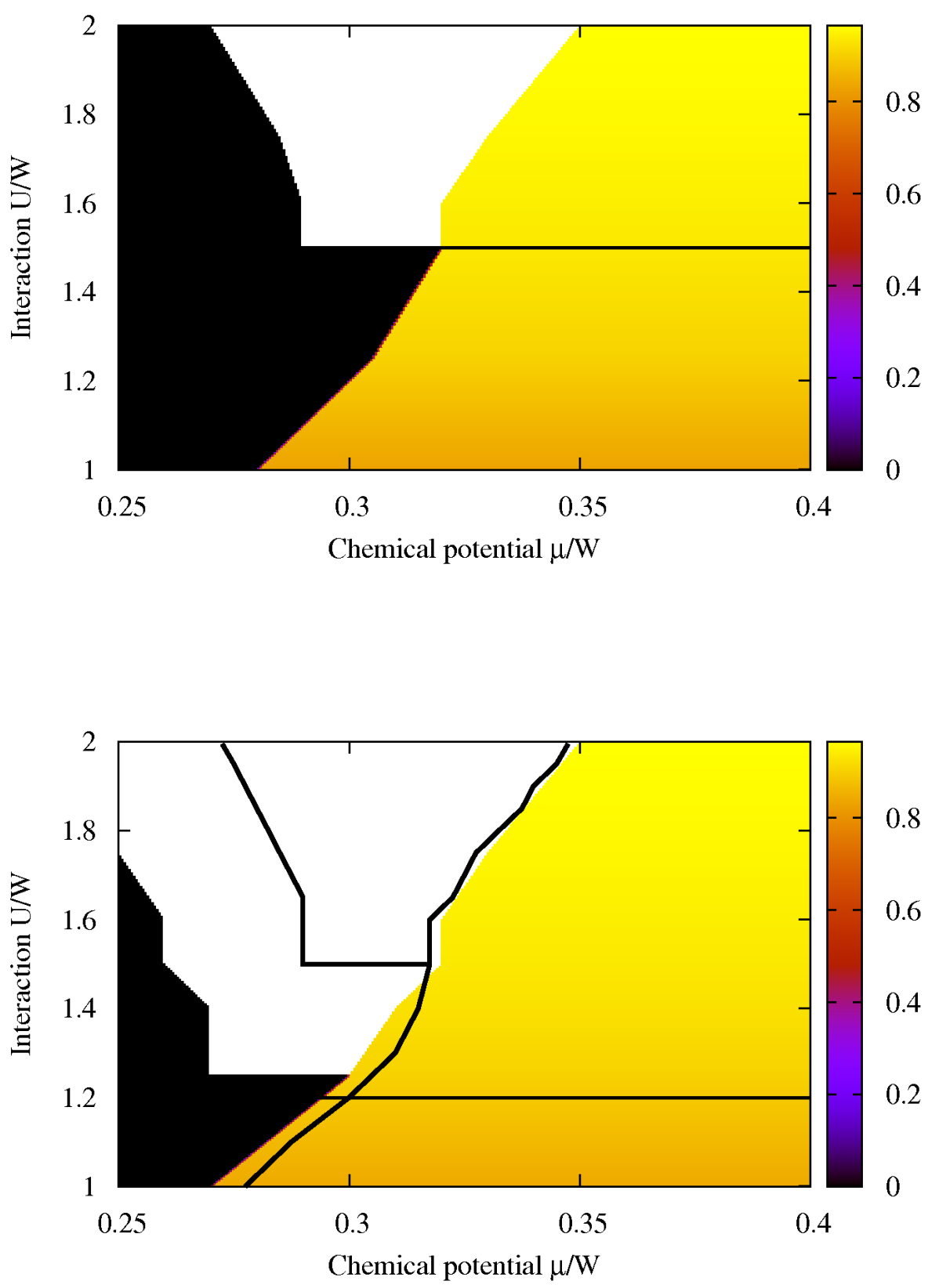

Figure 5.2: Antiferromagnetic polarization for a semi-elliptic DOS and $T / W=2 \cdot 10^{-4}$. The phase diagram was calculated with metallic starting medium in the upper panel (insulating starting medium in the lower panel). The antiferromagnetic Néel-phase (yellow color) exists only exactly at half-filling. Black is the paramagnetic phase away from half-filling and the white part the incommensurate spin density wave (IC). The horizontal black lines correspond to the critical values of $U / W=1.5$ (upper panel) and $U / W \approx 1.2$ (lower panel) of the PMIT. In the lower panel the transition lines of the calculation with metallic starting medium are included. The phase diagrams were created by fitting of approximately 200 data points distributed in each phase diagram. 
the existence of this ferromagnetic state. This stresses the influence of the lattice on the DMFT calculations.

The phase diagram for very low temperature $T / W=2 \cdot 10^{-4}$, different interaction strengths and chemical potentials can be seen in figure 5.2. Both panels show the antiferromagnetic polarization $p=n_{\uparrow}-n_{\downarrow}$, which is colored yellow corresponding to magnitudes larger than $p>0.8$. These phase diagrams were created by using different starting media for the DMFT calculation. The upper panel in figure 5.2 corresponds to a metallic starting medium, while the lower panel corresponds to an insulating starting medium. The media influence the critical value of the interacting strength $U / W$ at which the metal insulator transition occurs in the paramagnetic phase (figure 5.1). In both phase diagrams the antiferromagnetic phase exists only exactly at half-filling. This Néel-state is pinned to $n=1$ and does not change upon slightly changing the chemical potential. Looking at the metallic starting medium, the paramagnetic metal insulator transition would occur for $U / W \approx 1.5$, which is now covered by the antiferromagnetic state. Nevertheless, the magnetic phase diagram changes at this point. For interaction strengths below this value, the paramagnetic phase, represented by the black color, adjoins directly the antiferromagnetic phase at half-filling. The transition is of first order as both occupation and polarization jump, as can be seen in figure 5.3. There exists no stable solution for occupations between $n \approx 0.85$ and $n=1$. This region can be interpreted as phase separated between a paramagnetic solution away half-filling and an antiferromagnetic solution at half-filling [142-144]. The situation completely changes for interaction strengths above the critical value of the paramagnetic metal insulator transition, $U / W=1.5$. If one changes the chemical potential $\mu$ for $U / W>1.5$, one will find a region of parameters where the DMFT calculations do not converge, neither to an antiferromagnetic state nor to a paramagnetic state. This regions lies between the paramagnetic phase and the antiferromagnetic phase at half-filling. An example for a DMFT calculation in this region can be seen in figure 5.4. Both occupation and polarization oscillate with the DMFT iteration, and no convergent solution can be found. If one uses for example Broyden mixing [152] during the DMFT self-consistency circle, one can find a meta-stable solution, for the doped antiferromagnetic state. But as one stops using the mixing technique, the solution starts to iterate away and soon begins to oscillate again. This phase is usually interpreted as an incommensurate spin density wave, as found in these regions earlier by other authors [145,153-156]. Changing the chemical potential leads to a phase transition from the Néelstate at half-filling to this incommensurate phase and later into a paramagnetic phase. The phase diagram is symmetric towards half-filling, due to the symmetric DOS. Therefore the same behavior can be seen for electron doping.

Although the PMIT is covered by the antiferromagnetic phase, it is an interesting observation that it can be seen in the magnetic phase diagram, as at this point the phase separated region vanishes and the incommensurate magnetic phase emerges. This shows the close connection between both phenomena. That this transition occurs at the same interaction values is not by chance, can be seen in the lower panel of figure 5.2. The antiferromagnetic phase diagram depends on the starting medium, too. The lower panel shows the same calcula- 
tions as before, but for an insulating starting medium. The critical interaction strength for the PMIT lies at $U / W \approx 1.2$. The transition in the magnetic phase diagram between the phase separated region and the incommensurate phase occurs now at an interaction strength slightly above $U / W=1.2$. This discrepancy between both critical values of the interaction strength can be most likely explained by a finite resolution effect in the data points. I have additionally included the transition lines of the antiferromagnetic and the incommensurate phase with metallic starting medium into the figure. So one can very nicely see, how the incommensurate phase starts at smaller interaction strengths. One should notice, that for weak interaction strengths there is a hysteresis region, where one can obtain either an antiferromagnetic solution at half-filling or a paramagnetic solution away from half-filling. This is a common feature for a first order transition and can be seen in more detail in figure 5.3. The interaction value, at which the transition occurs, depends on the starting medium.

The incommensurate spin density state (IC) exists for temperatures below $T / W \approx 0.01$, see figures 5.6 and 5.5. If there is a close relation between the transition temperature of the IC phase and the critical temperature of the PMIT is difficult to determine, as the temperature resolution of the data points is bad in this region. Interestingly, the transition value between the phase separated and the IC phase seems to remain at $U / W \approx 1.5$ for increasing temperature, while the PMIT interaction value moves towards smaller values of $U / W$. When increasing the temperature it eventually becomes possible to dope the antiferromagnetic phase. This can be seen in the color gradient in figures 5.5 and 5.6, signaling a decreasing polarization, at the antiferromagnetic phase boundaries. The reason, why the IC-boundary remains at $U / W \approx 1.5$, is most likely due to this effect. The phase boundaries are smeared out and a doped antiferromagnetic phase is stabilized by increasing the temperature. For a temperature $T / W=0.03$, figure 5.6, the IC phase has completely vanished and the antiferromagnetic polarization smoothly decreases from half-filling towards its phase boundary away from half-filling.

\subsubsection{Spin Density Waves}

In the last section I have shown that regions of non-convergent DMFT calculations exist in the magnetic phase diagram. I have interpreted these regions as incommensurate spin density waves, as evidence for these were given by other authors $[145,153-156]$. I will show here, that it is in principle possible to stabilize such phases for a Bethe lattice with NN-hopping. In the theoretical part on the Bethe lattice in chapter 2.5, I have used the following equation for deriving the local Green's function $G_{00}(\zeta)$

$$
G_{00}^{-1}(\zeta)=\zeta-t^{2} \sum_{i \in N N} G_{i i}^{(0)}(\zeta) \rightarrow \zeta-\left(t^{\star}\right)^{2} G_{00}(\zeta)
$$

where $t$ is the hopping amplitude, $z$ the coordination number of the lattice, and the scaling $t=: \frac{t^{\star}}{\sqrt{z}}$ for $z \longrightarrow \infty$ holds. One assumes a homogeneous lattice, 


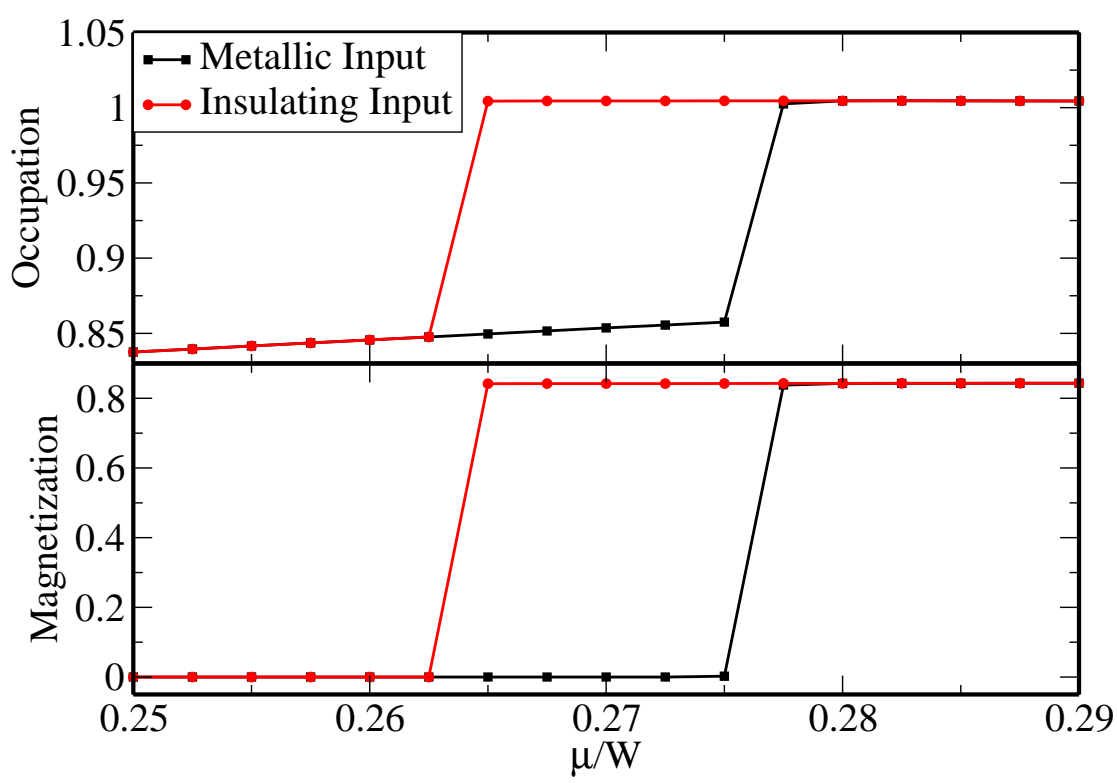

Figure 5.3: First order transition from the paramagnetic state away from half-filling to the antiferromagnetic Néel-state at half-filling for $T / W=2 \cdot 10^{-4}, U / W=1$ and two different starting media in the DMFT calculation.

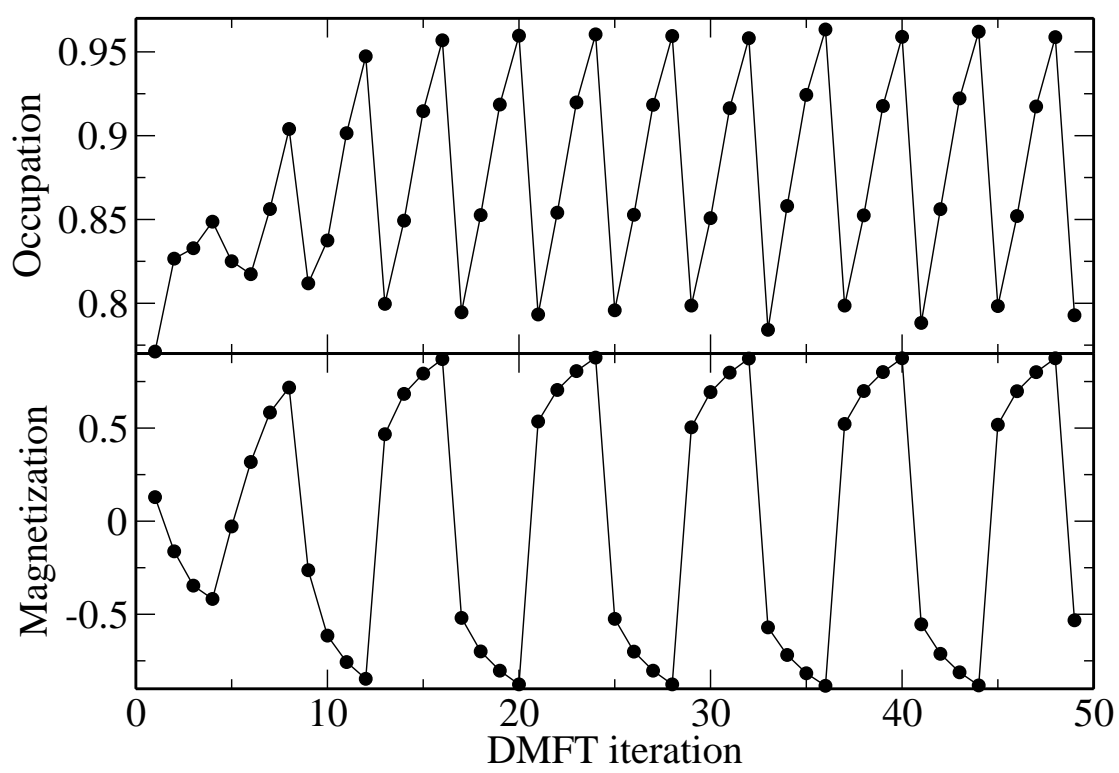

Figure 5.4: Non-convergent DMFT calculation for $T / W=2 \cdot 10^{-4}, U / W=2$, and $\mu / W=0.28$. The occupation and magnetization oscillate with the iteration number. No convergent state can be found. 


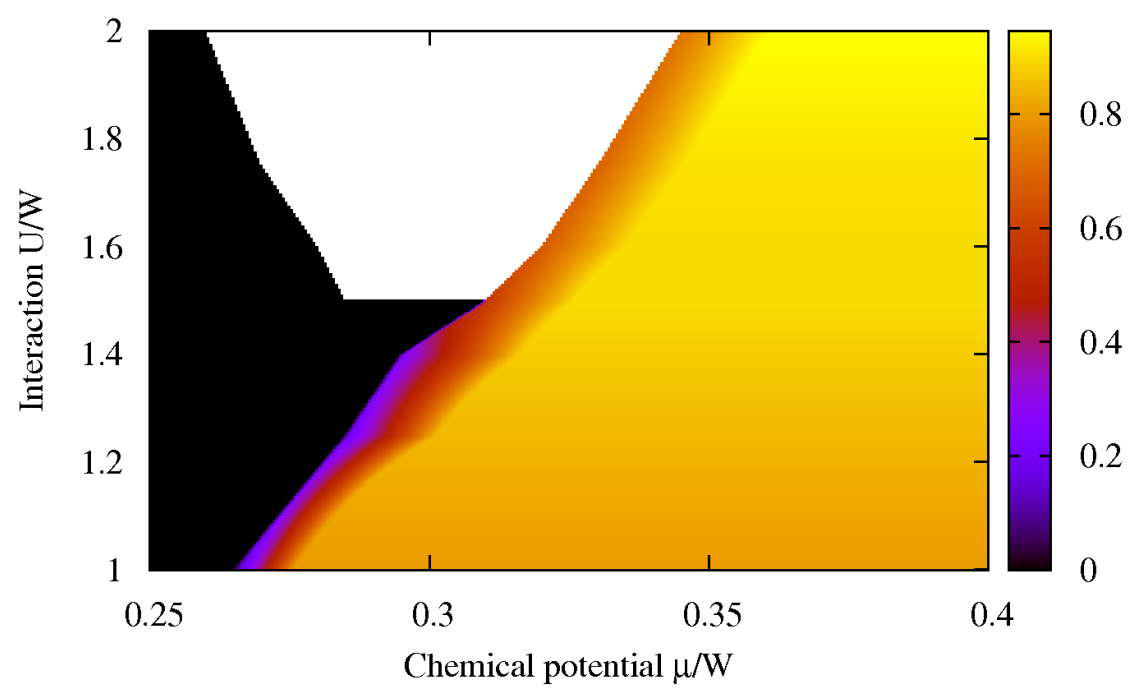

Figure 5.5: Magnetic phase diagram for $T / W=0.007$. The IC phase still exists for $U / W>1.5$. For small interaction strengths the jump of the magnetization at the phase boundary vanishes and there is now a smooth transition.

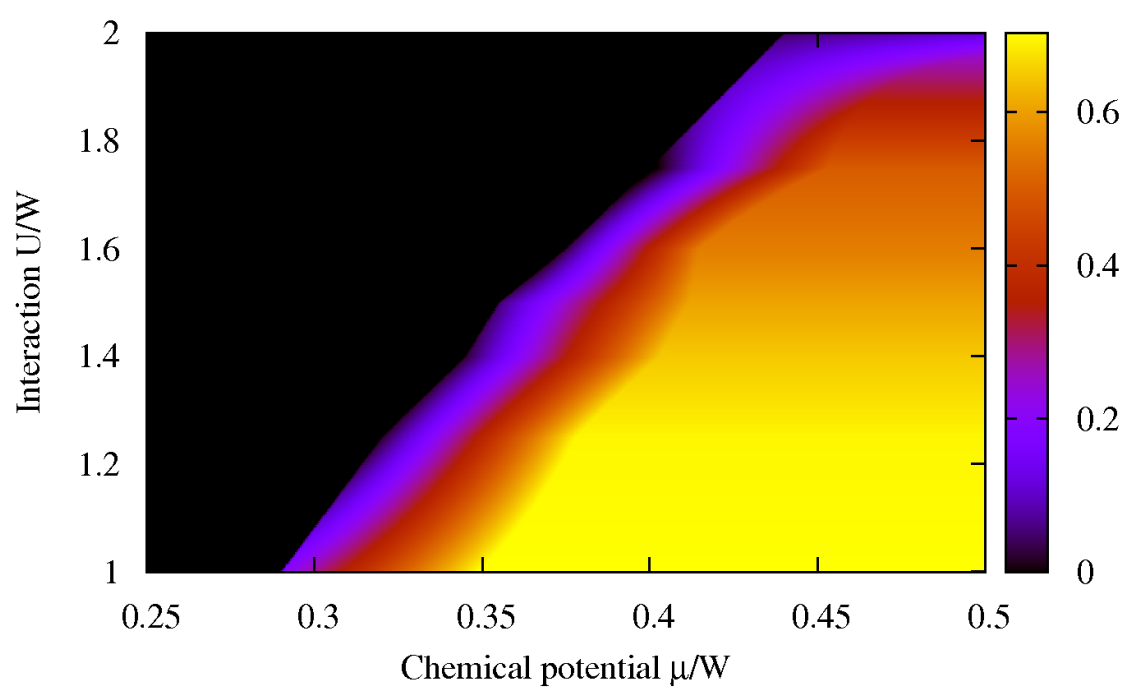

Figure 5.6: Magnetic phase diagram for $T / W=0.03$. The IC phase has completely vanished. 


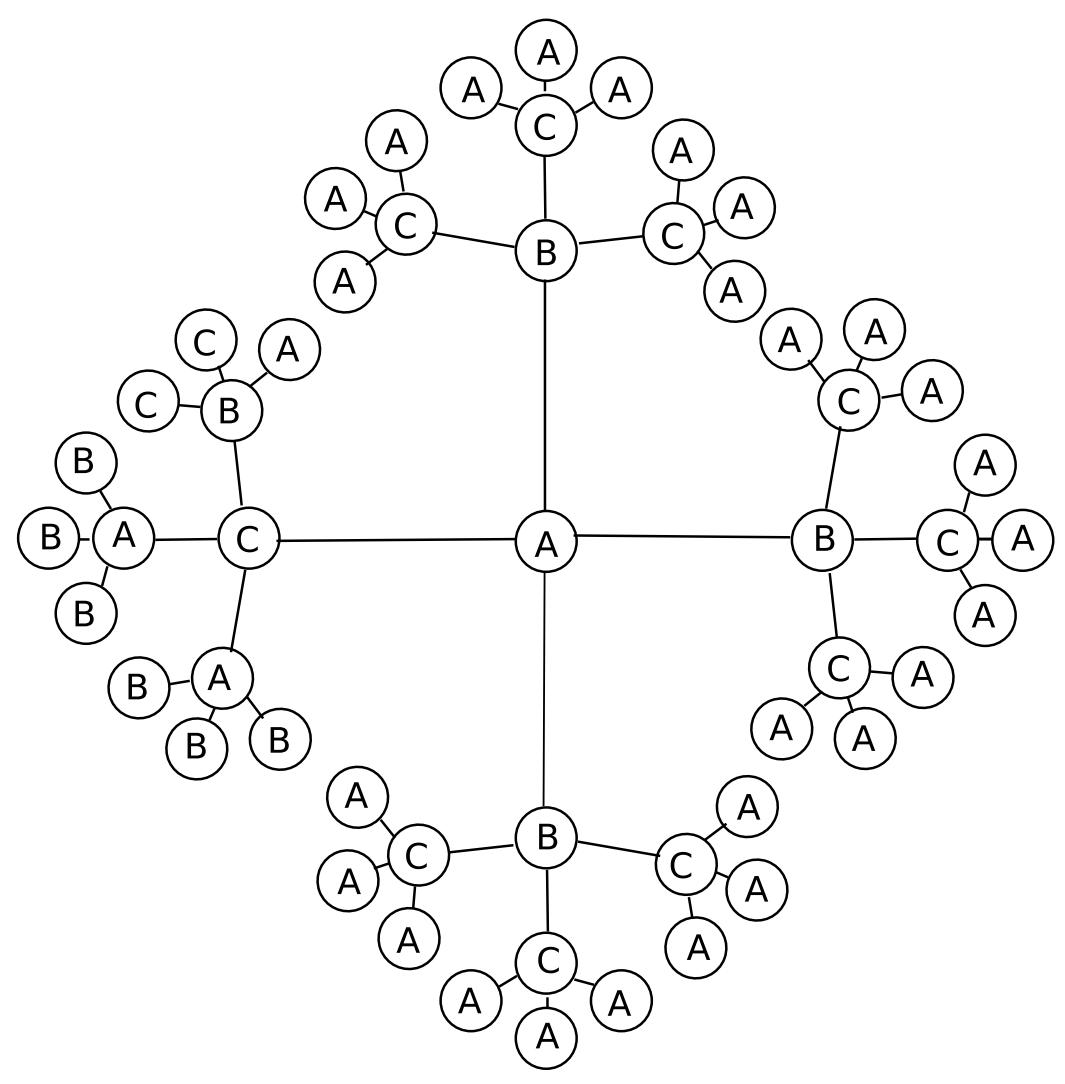

Figure 5.7: Bethe lattice with coordination number $z=4$ and a ABC-structure. Each A-site contacts three B-sites and one C-site.

which leads to $G_{i i}^{(0)}(\zeta)=G_{00}(\zeta)$. One can now assume a phase with broken symmetry, in which neighbors have different self-energy.

For a bipartite lattice one can immediately write down the equations for an ABstructure, because an A-site has only B-neighbors and vice versa. For longer periods it is more difficult to find a lattice division supporting, for example, an ABC-structure. But it is possible to define such structures for a Bethe lattice, where A-sites have direct contact to almost only B-sites and all Bsites contact almost only C-sites and so on. Almost only means that each A-site contacts one C-site and $z-1$ B-sites (see figure 5.7). From this lattice structure one can now set up the equation for each lattice site using equation (5.1). For a finite coordination number the sum over the nearest neighbors in equation (5.1) has contributions of two different Green's functions. But in the limit $z \longrightarrow \infty$ and the proper scaling of the hopping parameter there remains only one contribution. So there are $N$ equations for $N$ different local Green's 


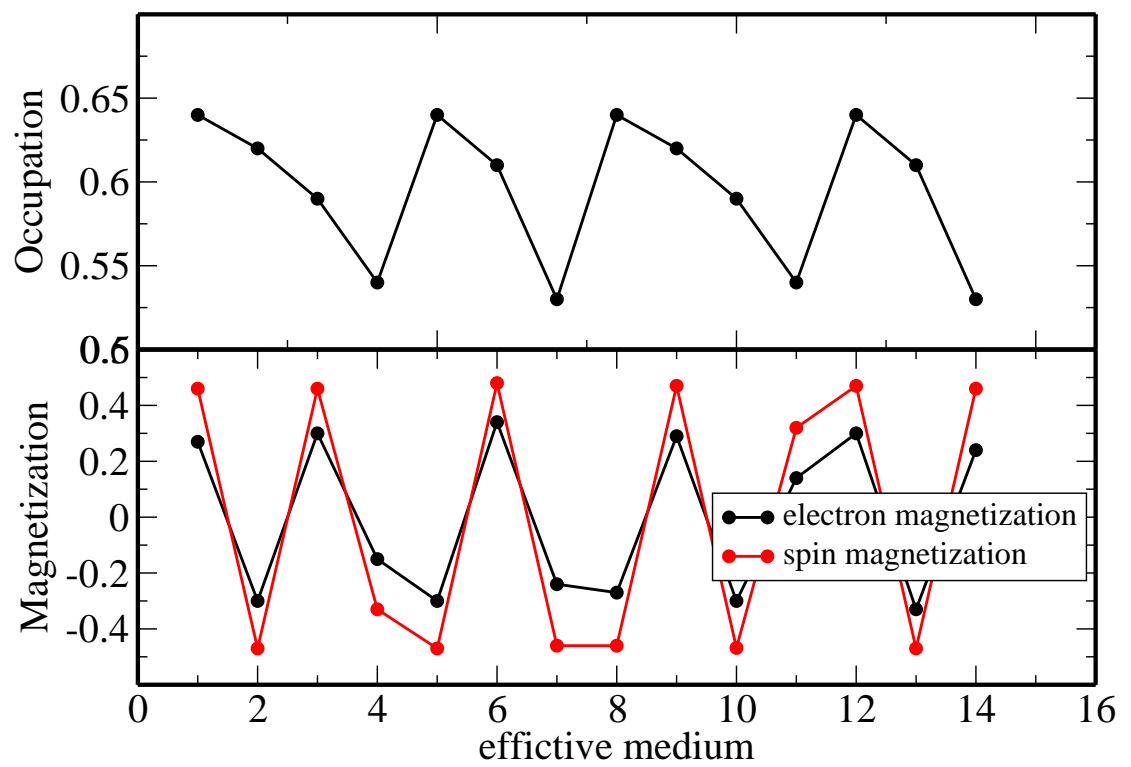

Figure 5.8: Stable spin density state away half-filling in the Kondo-lattice model with period $N=14$. The upper panel shows the occupation for each of the 14 lattice sites. The lower panel shows the magnetization of the electrons and of the spins.

functions, which define a closed set of equations

$$
\begin{aligned}
G_{1}^{-1}\left(\zeta_{1}\right) & =\zeta_{1}-\left(t^{\star}\right)^{2} G_{2}\left(\zeta_{2}\right) \\
G_{2}^{-1}\left(\zeta_{2}\right) & =\zeta_{2}-\left(t^{\star}\right)^{2} G_{3}\left(\zeta_{3}\right) \\
G_{3}^{-1}\left(\zeta_{3}\right) & =\zeta_{3}-\left(t^{\star}\right)^{2} G_{4}\left(\zeta_{4}\right) \\
& \vdots \\
G_{N}^{-1}\left(\zeta_{N}\right) & =\zeta_{N}-\left(t^{\star}\right)^{2} G_{1}\left(\zeta_{1}\right) .
\end{aligned}
$$

This can be solved yielding $N$ local Green's functions from which the new media for the next DMFT iteration are calculated. For an AB-structure this procedure yields the same new media as the matrix inversion shown in chapter 2.4.2 about DMFT.

Unfortunately, one cannot tell from a non-convergent solution, what is the correct period stabilizing the solution. Even though one can read off such a period in figure 5.4, this does not mean that using this will work. This means that one has to try different periods $N$ and hope to find the correct one. For one DMFT iteration for periodicity $N$, one has to solve $N$ different Anderson models. This makes long periods a heavy numerical task. By the end of this thesis I did not succeed in stabilizing a spin density wave in the Hubbard model for strong interaction. But it was possible in another project to stabilize such state in the Kondo-lattice model within DMFT. In this model there is a local spin $S$, which is coupled to the electrons via a spin-spin interaction with amplitude $J$, instead of a local density-density interaction. The model can be 
written as

$$
H=\sum_{i, \sigma} t_{i, j} c_{i, \sigma}^{\dagger} c_{j, \sigma}-\mu \sum_{i, \sigma} n_{i, \sigma}+J \sum_{i} \vec{S}_{i} \cdot \vec{s}_{i}
$$

where $\vec{s}$ is the spin operator for the electrons at each site. Also in this model there is an antiferromagnetic phase at half-filling, which eventually becomes unstable towards a spin-density wave [157] (and references within). Figure 5.8 shows an example for a stable spin density wave for $J / W=0.5, \mu / W=-0.225$, $T \approx 0$, and periodicity $N=14$. One should notice that the occupation and magnetization seems to oscillate with a period of $N \approx 3$, which however did not stabilize the system.

The example given shows that such order is possible. Nevertheless, the method used for stabilizing such states cannot be recommended, as the numerical effort is very high. Additionally, such solutions seem to be rather unstable towards numerical errors, making it very hard to find them.

\subsection{Comparison of DMRG and NRG}

In principle the DMRG is an alternative to the NRG as impurity solver for the DMFT [115-118]. For the Bethe lattice it is possible to formulate the DMFT self-consistency equation as [70]

$$
\mathcal{G}^{-1}(z)=t^{2} G(z)
$$

in which $t$ is the hopping parameter in the Hubbard model, $G(z)$ the impurity Green's function and $\mathcal{G}$ the effective medium of the DMFT. Hence, for the Bethe lattice it is not necessary to calculate the self-energy for determining the effective medium. As it is simpler within the DMRG to calculate the impurity Green's function than calculating the self-energy, I used this formulation of the self-consistency. In the DMRG one usually calculates $G(\omega+i \eta)$. Even though the self-consistency can be formulated for arbitrary $\eta$, for determining the hopping parameters from the effective medium, which are needed in the next DMRG calculation, one needs $\mathcal{G}^{-1}(\omega+i 0)$. Therefore a deconvolution must be performed after every impurity calculation. The PMIT was analyzed within the DMFT and DMRG as impurity solver in several works [158-160]. They showed that it is possible to use DMRG as impurity solver for DMFT and discussed spectral features close to the Mott transition. I will here concentrate on magnetic phases. Because the antiferromagnetic phase at half-filling is the most pronounced magnetic phase in the one-orbital Hubbard model, I will concentrate on this state.

Figure 5.9 shows the antiferromagnetic DMFT results for $U / W=\{0.5 ; 0.8$; $1.0 ; 1.5\}$ and half-filling. The solutions were calculated separately with the DMRG and the NRG. The DMRG was performed for a bandwidth of $W=0.2$ and a broadening $\eta=0.05$. The shown DMRG results are already deconvoluted. For the NRG calculations, I used the same bandwidth and the usual frequency dependent broadening with $b=0.8$, as described in chapter 3.3.3. For $U / W=0.05$ (upper left panel) the antiferromagnetic gap is too small to be 


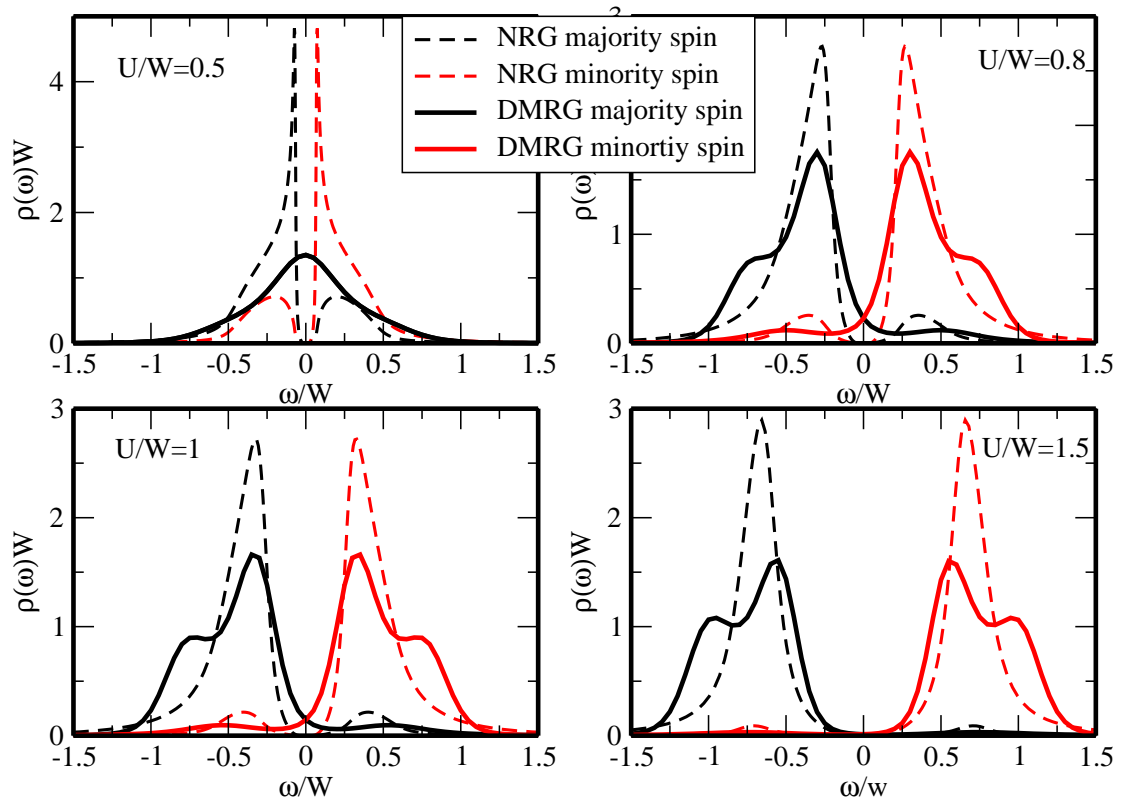

Figure 5.9: Antiferromagnetic DMFT calculations performed with DMRG and NRG as impurity solver for different interaction strengths $U / W$. The DMRG results are already deconvoluted. For $U / W=0.5$ the gap is to small to be resolved by the DMRG. Thus the DMRG failed to stabilize the antiferromagnetic phase.

resolved by the DMRG. The gap-width for this interaction strength, as calculated by the NRG, is $\Delta_{A F} \approx 0.015$. With the mentioned Lorentzian broadening this cannot be resolved and is too small even after deconvolution. Thus the solution of the DMFT with DMRG for this interaction is a half-filled paramagnetic metal. In contrast, the NRG can resolve this gap resulting in an antiferromagnetic insulator with strong van-Hove singularities at the gap edges. For stronger interactions DMFT with DMRG can stabilize the correct solution, meaning an antiferromagnetic state. If one looks carefully, one will notice that even if there is an antiferromagnetic solution for $U / W=0.8$ and $U / W=1.0$, DMRG fails to really open the gap. The DMRG results would actually predict an antiferromagnetic metallic state for these intermediate interactions. The reason for this is again the broadening. The gap-width in both system has the same order of magnitude as the broadening $\eta$. Only for stronger interactions $U / W>1.5$ one can clearly see a gaped antiferromagnetic state in the DMRG calculations. The interaction strengths, at which the DMRG is able to open the gap and form an antiferromagnetic solution, depend on the ratio $\eta / W$. Using smaller broadening will make it possible to better resolve the low frequency parts including the antiferromagnetic gap. But using a smaller broadening $\eta$ will, of course, result in a heavier numerical task.

Another big difference between the spectral functions of the NRG and the DMRG is the structure of the Hubbard bands. As the broadening in the NRG is large for large frequencies, the Hubbard bands do not show additional structures for $U / W \geq 0.8$. In the NRG one can only see for $U / W=0.5$ van-Hove 


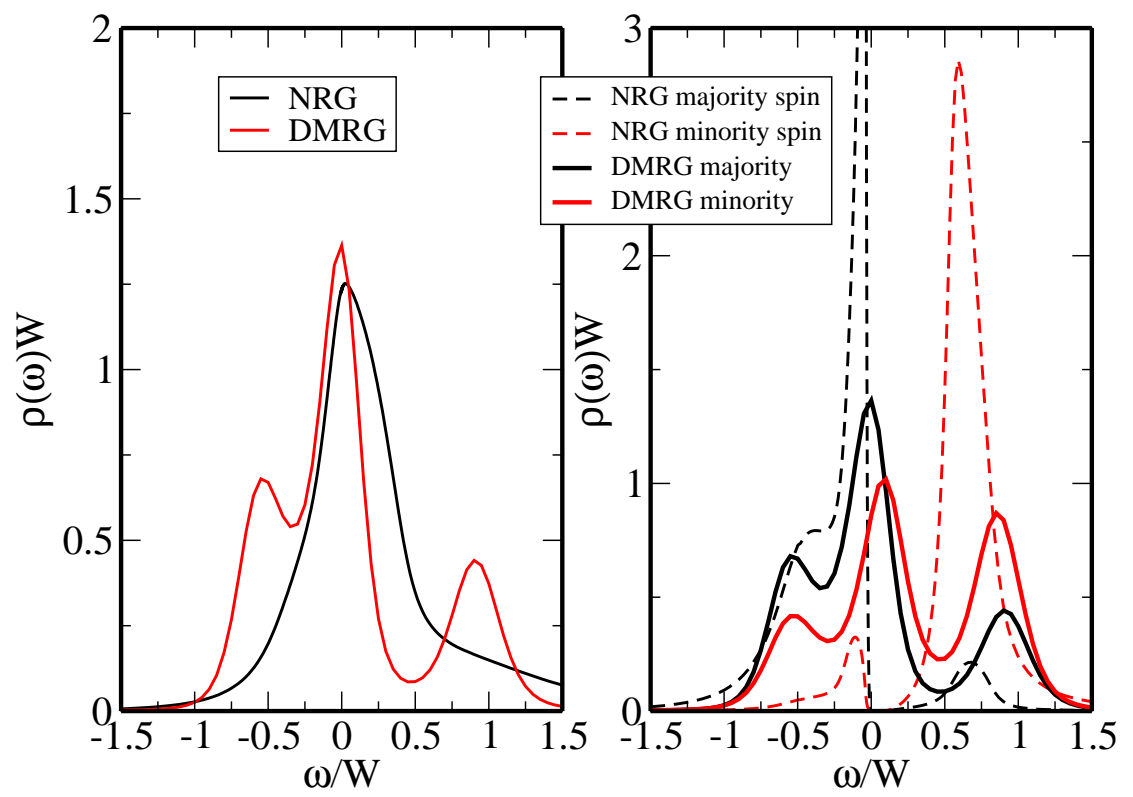

Figure 5.10: DMRG and NRG doped spectral function for $U / W=1$ and $T \approx 0$. The left panel shows the paramagnetic solution for $\mu / W=0.1$. The right panel shows antiferromagnetic solutions for $\mu / W=0.3$.

singularities and a shoulder in the Hubbard bands. The DMRG results include always two structures in each Hubbard band. There are theoretical results on additional structures in the antiferromagnetic state [153,161-164]. These additional structures are created by virtual movement of electrons or holes in long paths. If the structures found here are related to this must be further investigated. It is possible that they are only artifacts of the interplay between broadening and deconvolution. All shown results in figure 5.9 were calculated using the same broadening $\eta=0.05$. One should do the same calculations using different $\eta$. If the structures remain at their positions, they are most likely of physical origin.

Besides the antiferromagnetic behavior for different interaction strengths, one should also compare the doping behavior. Figure 5.10 shows the spectral functions of antiferromagnetic calculations for two different chemical potentials $\mu / W=0.1$ and $\mu / W=0.3$. For $U / W=1$ the system jumps directly from a paramagnetic metal away from half-filling into an antiferromagnetic insulator at $\mu / W \approx 0.27$, as I have discussed above. Notice that although the NRG solution in the left panel of figure 5.10 is half-filled, it is asymmetric. The DMFT/DMRG calculations show a change in their behavior at approximately the same value of the chemical potential. In contrast to the NRG results, DMRG stabilizes a metallic antiferromagnetic state away half-filling. The filling of the system is $\langle n\rangle \approx 0.9$ and lies therefore exactly in the phase separated region in the DMFT/NRG calculations. Although one cannot be completely sure, there is strong evidence for phase separation between the paramagnetic metal and the antiferromagnetic insulator in this parameter regime [142-144]. The reason for 
the stable antiferromagnetic metal is most likely again the interplay between the broadening and deconvolution.

\subsection{Frustrated Bethe Lattice}

\subsubsection{Half-Filling}

I will now introduce frustration to the antiferromagnetic state. Frustration is one of the main arguments when explaining, why the PMIT reaches out of the antiferromagnetic dome in $\mathrm{V}_{2} \mathrm{O}_{3}$ (chapter 1.2.1). This leads to the situation that not all interactions can be saturated. The simplest example is a triangle, in which all bonds are antiferromagnetic. If two of the three sites are in a Néelstate the third site has a bond to an up-site and one to a down-site. Therefore it cannot saturate both bonds. $\mathrm{V}_{2} \mathrm{O}_{3}$ crystallizes in a corundum structure, which is a frustrated three-dimensional lattice [14].

The calculations performed here are done for a Bethe lattice with NN- and NNN-hopping. Including both hopping terms into the Hamiltonian creates triangles in the lattice (see chapter 2.5). The major difference within the DMFT to NN-hopping only is that the DOS becomes now asymmetric and develops a van-Hove singularity at the lower band edge for $t_{2} / t_{1}>1 / 4$. I will first analyze the results for half-filling. There were already calculations within DMFT for a frustrated Bethe lattice. But most of the old calculation used the so called two-sublattice frustrated model $[70,147,148,165-167]$, which results in a particle-hole symmetric DOS even with frustration. As side effect, this way of introducing frustration leaves the paramagnetic phase unchanged. For the frustrated antiferromagnetic ordered system, on the other hand, there exists a lower critical value for the interaction $U$, which increases with increasing frustration. It was furthermore found that the Néel-temperature decreases with increasing frustration such that the PMIT eventually outgrows the antiferromagnetic phase [147]. In early calculations using this way of introducing frustration based on exact diagonalization studies or Hartree-Fock of the two-sublattice fully frustrated model $[70,166,167]$, the authors also found parameter regions in the phase diagram, where an antiferromagnetic metal appeared to be stable. However, this antiferromagnetic metallic phase was later traced back to numerical subtleties in the exact diagonalization procedure and shown to be actually absent from the phase diagram [147].

The first attempt to study the Hubbard model for the Bethe lattice with the correct inclusion of NN- and NNN-hopping has been performed rather recently in 2007 by M. Eckstein et al. [168] within the self-energy functional approach [169]. The authors particularly focused on the PMIT for $t_{2} / t_{1}=3 / 7$ and found phase separation between the insulating and metallic phase.

In this part of the work I will investigate the PMIT as well as the antiferromagnetic phase at half-filling and concentrate on the competition between the paramagnetic phase including the PMIT and the antiferromagnetic phase at intermediate and high grades of frustration. I especially look at the case $t_{2} \rightarrow t_{1}$ and raise the question, if the scenario of the outgrowing PMIT, proposed in [147], still holds for the correct asymmetric density of states. The cal- 


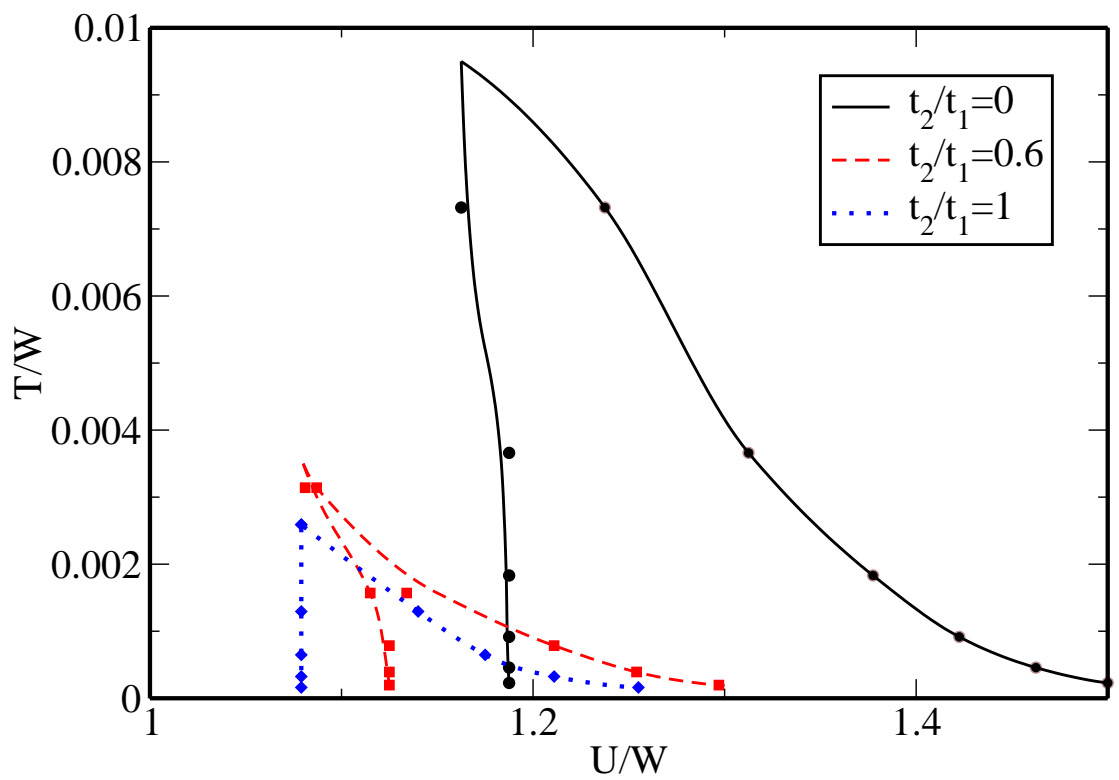

Figure 5.11: The transition lines for the PMIT for different frustrations as function of temperature and interaction strength. For each frustration the right line represents the transition from the metal to the insulator, while the left line represents the transition from the insulator to the metal. Symbols mark the calculated data points, the lines are fits meant as guide to the eye.

culations were done using Wilson's NRG as the impurity solver for the DMFT, with $\Lambda=2, N_{K}=1800$ states kept per NRG step and a logarithmic broadening, $b=0.8$, to obtain spectral functions. Note that the paramagnetic results are obtained by artificially suppressing an antiferromagnetic instability. The occupation was kept fixed at $n=1 \pm 0.005$ by adjusting the chemical potential. In contrast to the case with $t_{2}=0$ it is not possible to achieve $n=1$ here within numerical precision due to the asymmetric DOS. For increasing $t_{2} / t_{1} \rightarrow 1$ the PMIT is shifted towards lower interaction strengths and also lower temperatures, see figure 5.11. While the shift in the interaction strength is rather moderate, there is a large difference in the temperature of the critical endpoint between the unfrustrated and highly frustrated system. A shift of the same magnitude was also reported by M. Eckstein et al. [168]. This observation, of course, renews the interest in the question, to what extent long-range hopping can help to push the paramagnetic MIT out of the expected antiferromagnetic phase for reasonable magnitudes of $t_{2}$ to create a phase diagram similar to the one found for $\mathrm{V}_{2} \mathrm{O}_{3}$. The scenario proposed by R. Zitzler et al. [147] relied on the fact that the paramagnetic phase largely remains unaltered with increasing $t_{2}$. As the Néel-temperature for the antiferromagnet is reduced at the same time, the PMIT can eventually outgrow the antiferromagnetic phase. Due to the reduction of the critical point of the PMIT this must be analyzed more carefully now. 


\subsubsection{Antiferromagnetism at half-filling}

I will now allow for antiferromagnetic ordering in the calculations. Figure 5.12 shows the resulting phase diagrams for $t_{2} / t_{1}=0.6$ (upper panel) and $t_{2} / t_{1}=$ 0.8 (lower panel) for different temperatures and interaction strengths. The phase diagrams were constructed by fitting the magnetization to the actually calculated data points. This of course means that the phase boundaries shown here must be considered as guess only. However, as one does not expect any strange structures to appear, this guess will presumably represent the true phase boundary within a few percent. The full lines in figure 5.12 are the PMIT transitions. Note that for both diagrams the same division of axes was chosen.

In contrast to the Hubbard model for a bipartite lattice with $t_{2}=0$, there now exists a finite critical value $U_{c}^{A F}>0$, below which no antiferromagnetism can be stabilized even for temperature $T \longrightarrow 0$. With increasing frustration the paramagnetic-antiferromagnetic transition is shifted towards higher interaction strengths and lower temperatures, while the PMIT is shifted towards lower interactions strengths. So obviously the PMIT is shifted towards the phase boundaries of the antiferromagnetic dome. So far this is the expected effect of the NNN-hopping which introduces frustration to the antiferromagnetic exchange. However, note that although $t_{2} / t_{1}=0.8$ represents already a very strongly frustrated system, the PMIT still lies well covered within the antiferromagnetic phase.

I will now have a closer look at the paramagnetic-antiferromagnetic transition. Here, R. Zitzler et al. [147] made the prediction that one has to expect a first order transition close to the critical $U_{c}^{A F}$ at low temperatures; while at larger values of $U$ again a second order transition was found. Figure 5.13 shows the staggered magnetization for different temperatures and interaction strengths at fixed $t_{2} / t_{1}=0.8$. The upper panel collects data for the transition at low temperatures at the lower edge of the antiferromagnetic phase. The full lines represent the transition from the paramagnetic to the antiferromagnetic state with increasing interaction strength for two different temperatures, while the dashed lines represent the transitions from the antiferromagnetic to the paramagnetic state with decreasing interaction strength. In the upper panel (low $T$ ) one can clearly see a hysteresis of the antiferromagnetic transition. This hysteresis as well as the jump in the magnetization are clear signs for a first order transition. This antiferromagnetic hysteresis is very pronounced for strong frustration but numerically not resolvable for example for $t_{2} / t_{1}=0.2$. The hysteresis regions seems to shrink with decreasing $t_{2}$ and eventually cannot be resolved anymore with numerical techniques. Note that such a hysteresis is also found in the two-sublattice fully frustrated model [147], which means that this is quite likely a generic effect in frustrated systems at intermediate coupling strengths. The lower panel in Fig. 5.13 shows the staggered magnetization for temperatures just below the corresponding Néel-temperatures and at large interaction strengths. Here the magnetization vanishes smoothly, which is the behavior expected for a second order phase transition. In summary I thus find a first order transition at the critical interaction $U_{c}^{A F}$, where the antiferro- 

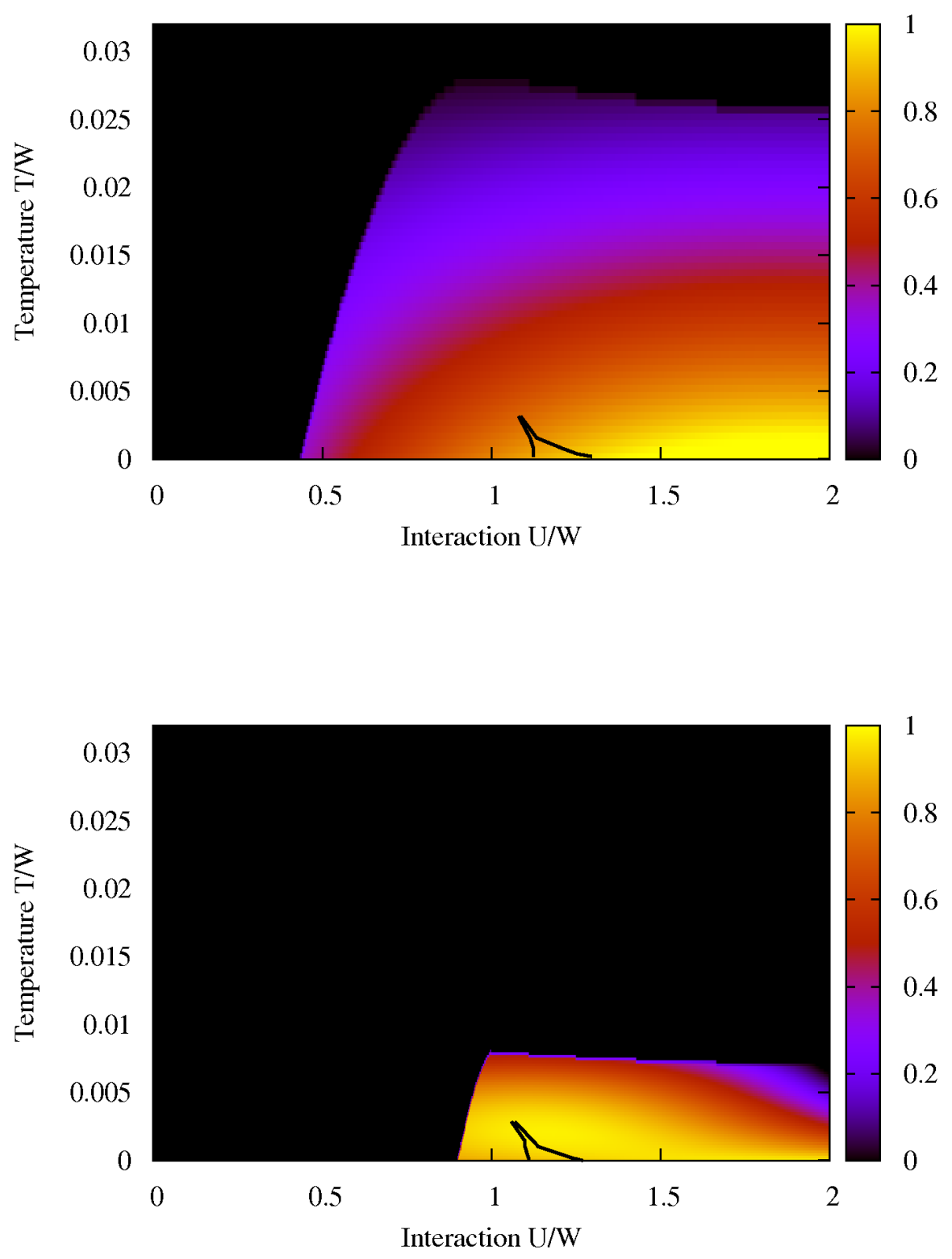

Figure 5.12: The upper (lower) panel shows the $T-U$ phase diagram for $t_{2} / t_{1}=0.6$ $\left(t_{2} / t_{1}=0.8\right)$. The colored area represents the antiferromagnetic phase, while the black area represents the paramagnetic phase. The lines show, where the PMIT in the paramagnetic phase would occur. The phase diagrams were constructed by fitting the magnetization to approximately 70 calculated data points. Additional calculations were performed to find the PMIT-lines. 

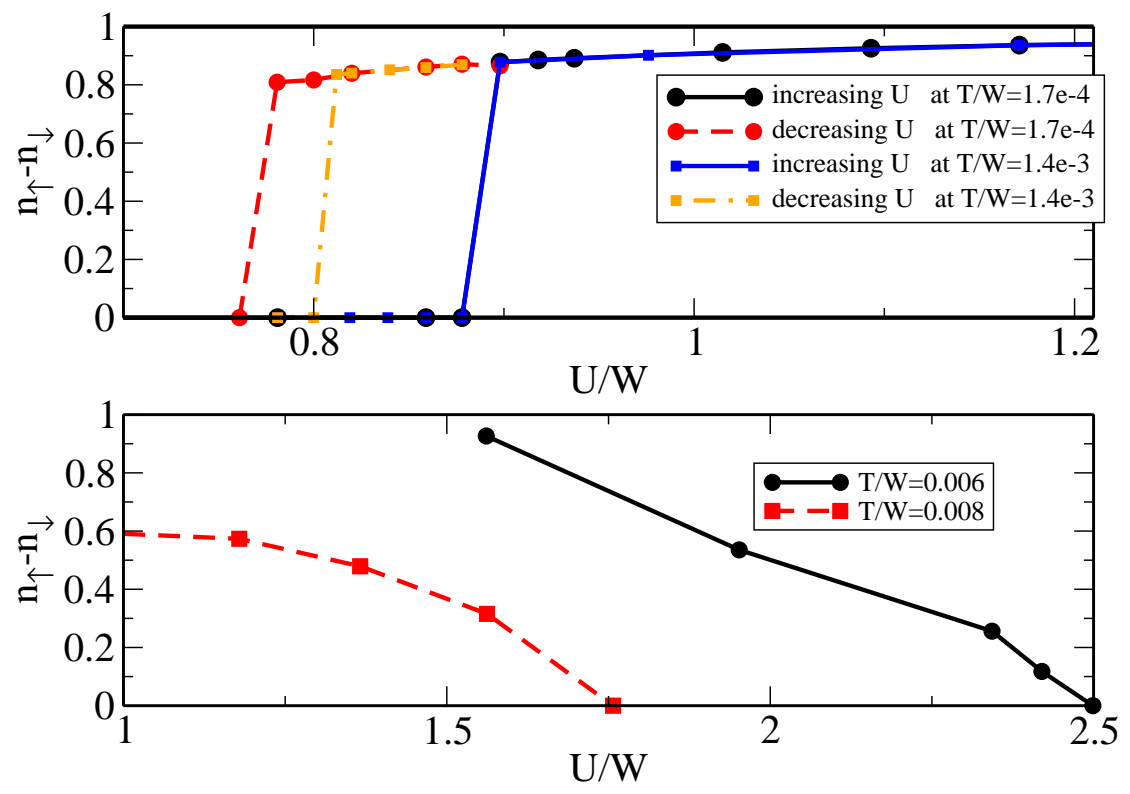

Figure 5.13: Staggered magnetization versus interaction $U / W$ for two different temperatures and $t_{2} / t_{1}=0.8$. In the upper panel there are two transition lines for each temperature, representing either increasing or decreasing interaction strength. The region between both lines embodies a hysteresis region. The lower panel shows the transition for large interaction strengths and high temperatures. Here no hysteresis region can be found, but a smooth transition. The lines are meant as guide to the eye.

magnetism sets in, and a second order transition for large Coulomb parameter $U \gg W$. The merging from both transition lines is an interesting point in itself. There must be a critical point where the first order transition changes into a second order transition. It is however not possible to resolve this merging within DMFT/NRG. The magnetization of the system becomes very small in this region, so it is not possible to distinguish between a (tiny) jump and numerical artifacts of a smoothly vanishing order parameter. Consequently, one cannot decide anymore of what order the transition is.

Antiferromagnetic metallic states at half-filling were reported in earlier publications [148, 165-167] and later ruled out again. In my calculations I saw no evidence for an antiferromagnetic metallic state at half-filling. Especially for strong frustration $t_{2} / t_{1} \approx 0.8$ the system jumps directly from a paramagnetic metallic solution into an antiferromagnetic insulating solution with large magnetization. In the papers cited, the region showing an antiferromagnetic metallic solution broadens with increasing $t_{2}$. This prediction I clearly cannot confirm, as discussed above. Only in systems with small to intermediate frustration there are narrow interaction regimes where I observe a small finite weight at the Fermi level. One must however consider that the occupation number is not exactly one but only within $0.5 \%$, which influences the position of the gap. It was also sometimes difficult to stabilize a DMFT solution in these regions. In summary, I cannot see any clear signs for an antiferromagnetic metallic state at half-filling in my calculations. If any exists, then only for rather low frustrations 
in a very small regime about the critical interaction $U_{C}^{A F}$. To what extent these rather special conditions can then be considered as realistic for real materials is yet another question.

\subsubsection{Nearly fully frustrated system}

In the last part concerning half-filling I want to study the situation, in which $t_{1}$ and $t_{2}$ are comparable in strength. Interestingly, there has been no attempt to calculate the phase diagram on a mean-field level in the strongly frustrated model $t_{2} \approx t_{1}$. Therefore, before discussing the results of the DMFT calculations for strongly frustrated systems let me try to gain some insight into the physics I must expect, by inspecting classical spins on a Bethe lattice with NNinteraction $J_{1}$ and NNN-interaction $J_{2}$. In this calculation I firstly assume a
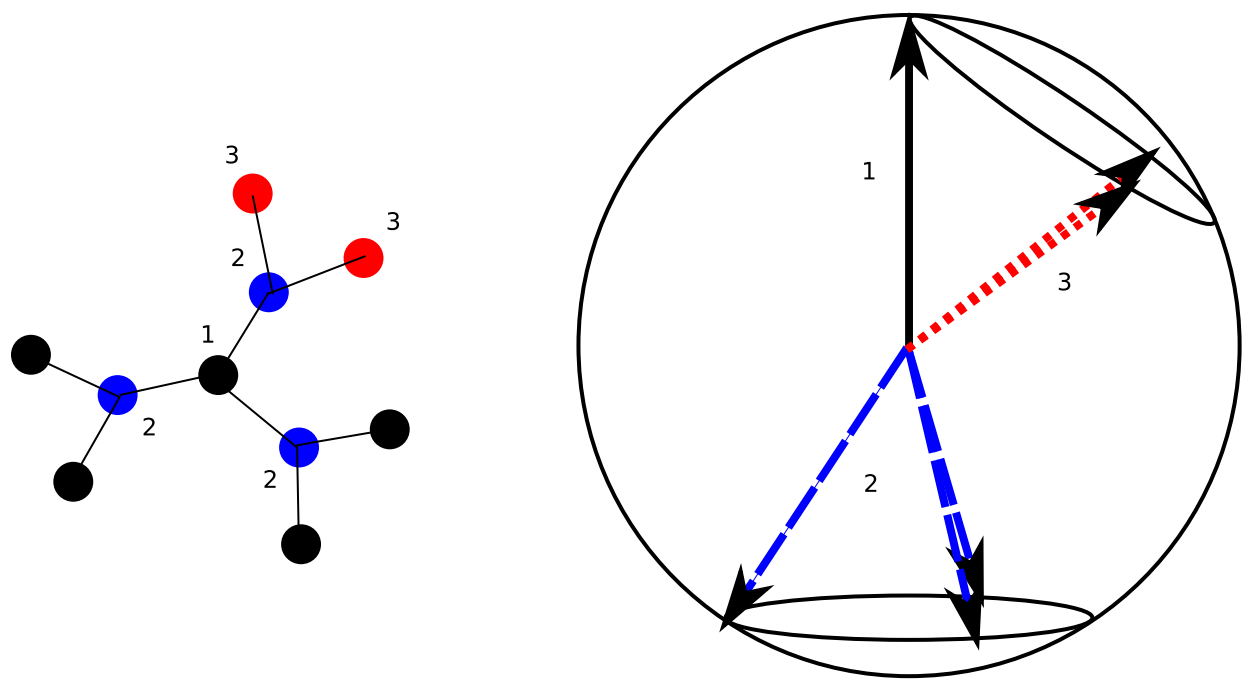

Figure 5.14: Left: Bethe lattice $z=3$ with sites numbered according to the vector spins on the right. The nearest neighbors of site 1 must lie on a circle so there is an angle $\theta$ between 1 and 2. Similarly the nearest neighbors of one of the 2 -spins must lie on a circle including the 3 -spins and the 1 -spin.

Bethe lattice with coordination number $z$. The last parameter entering is the angle $\theta$ between NN-spins. Although the initial assumption that two neighboring spins form an angle $\theta$ may seem somewhat restrictive, I am not aware of other configurations with lower total energy [170]. I want to minimize the energy with respect to this angle. According to figure 5.14 the NN-spins of one spin, must lie on a circle. The spins ending on the circle are all NNN-spins of each other. Due to the antiferromagnetic interaction $J_{2}$, one can assume that they want to maximize the angle between them. Since there are $z$ spins on each circle, they will have an angle $2 \pi / z$ projected on the circle. Using now simple trigonometry, the angle between NNN-spins $\gamma$ is given by

$$
\begin{aligned}
\cos \gamma & =\frac{2-\left(2 R^{2}-2 R^{2} \cos (2 \pi i / z)\right)}{2} \\
& =\cos (\theta)^{2}+\sin (\theta)^{2} \cos (2 \pi i / z)
\end{aligned}
$$




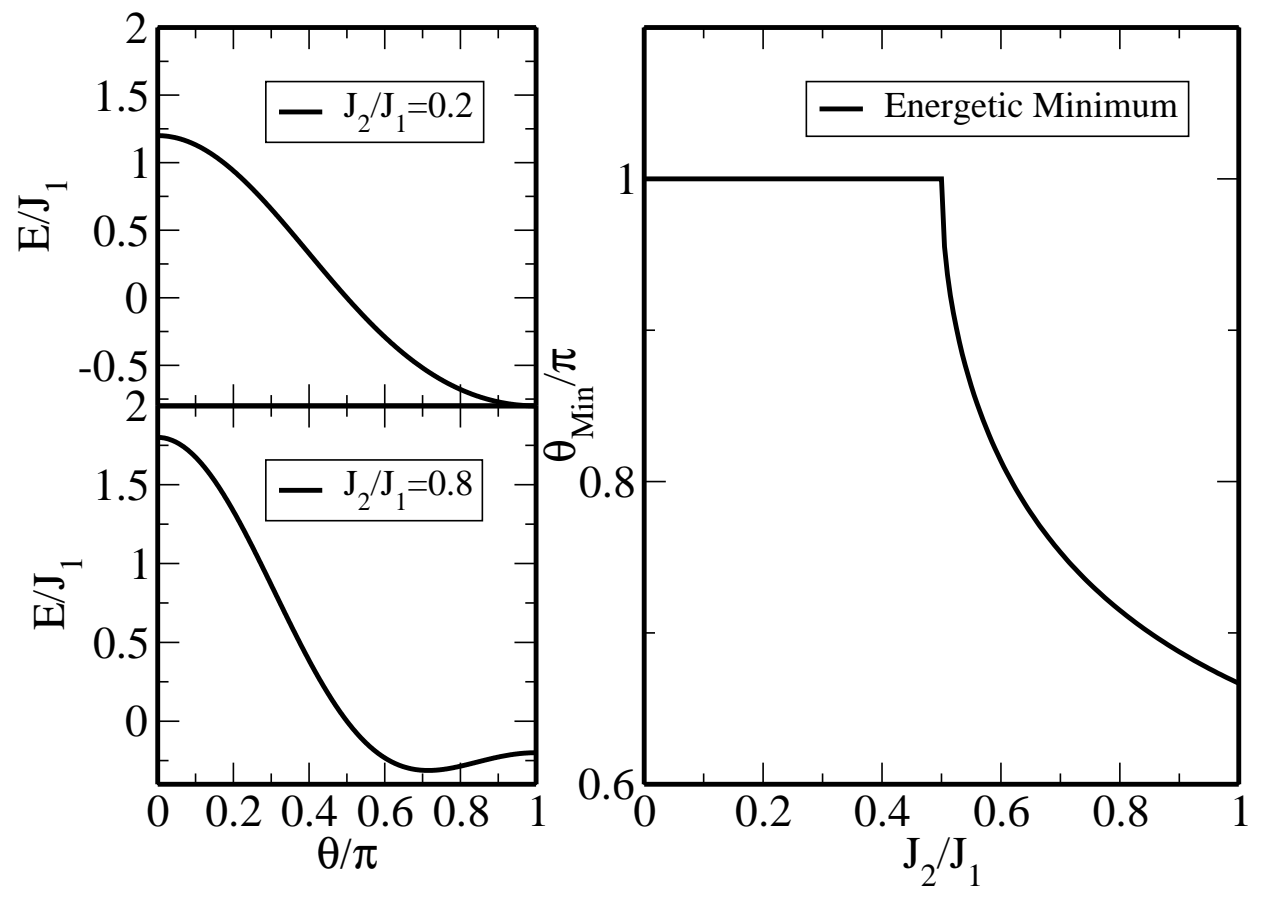

Figure 5.15: Left: Energy $E / J_{1}$ via the nearest neighbor angle $\theta$ for two ratios of $J_{2} / J_{1}$. Right: Nearest neighbor angle $\theta_{\text {Min }}$, which minimizes the energy for different ratios $J_{2} / J_{1}$

where $i$ runs from 0 to $z-1$, giving the different positions on one circle, and $R=\sin (\theta)$ is the radius of the NN-circle. Inserting this into the Hamiltonian

$$
E=J_{1} \sum_{i, j \in N N} \vec{S}_{i} \vec{S}_{j}+J_{2} \sum_{i, j \in N N N} \overrightarrow{S_{i}} \vec{S}_{j}
$$

one finds for the energy

$$
\begin{aligned}
E / 2 N & =J_{1} z \cos (\theta) \\
& +J_{2} z \sum_{i=1}^{z-1}\left(\cos (\theta)^{2}+\sin (\theta)^{2} \cos (2 \pi i / z)\right) .
\end{aligned}
$$

Performing now the limit $z \rightarrow \infty$ and scaling $J_{1} z \rightarrow J_{1}^{\star}$ and $J_{2} z \rightarrow J_{2}^{\star} / z$, as it is done for the hopping amplitudes in DMFT, one finally obtains for the energy per lattice site

$$
E_{z=\infty}(\theta) /(2 N)=J_{1}^{\star} \cos (\theta)+J_{2}^{\star} \cos (\theta)^{2} .
$$

The energy depending on $\theta$ can be seen in figure 5.15. If both interactions $J_{1}^{\star}$ and $J_{2}^{\star}$ are antiferromagnetic the ferromagnetic configuration of the spins represented by $\theta=0$ is always a maximum. The Néel-state $\theta=\pi$ is the stable minimum for $J_{2}^{\star} / J_{1}^{\star}<1 / 2$, because $d^{2} E(\theta=\pi) / d \theta^{2}=J_{1}^{\star}-2 J_{2}^{\star}$. The angle minimizing the energy for $J_{2}^{\star} / J_{1}^{\star}>1 / 2$ is found to be $\theta=\arccos \left(-\frac{J_{1}^{\star}}{2 J_{2}^{\star}}\right)$, corresponding to a periodically modulated spin state. In this very simplified 


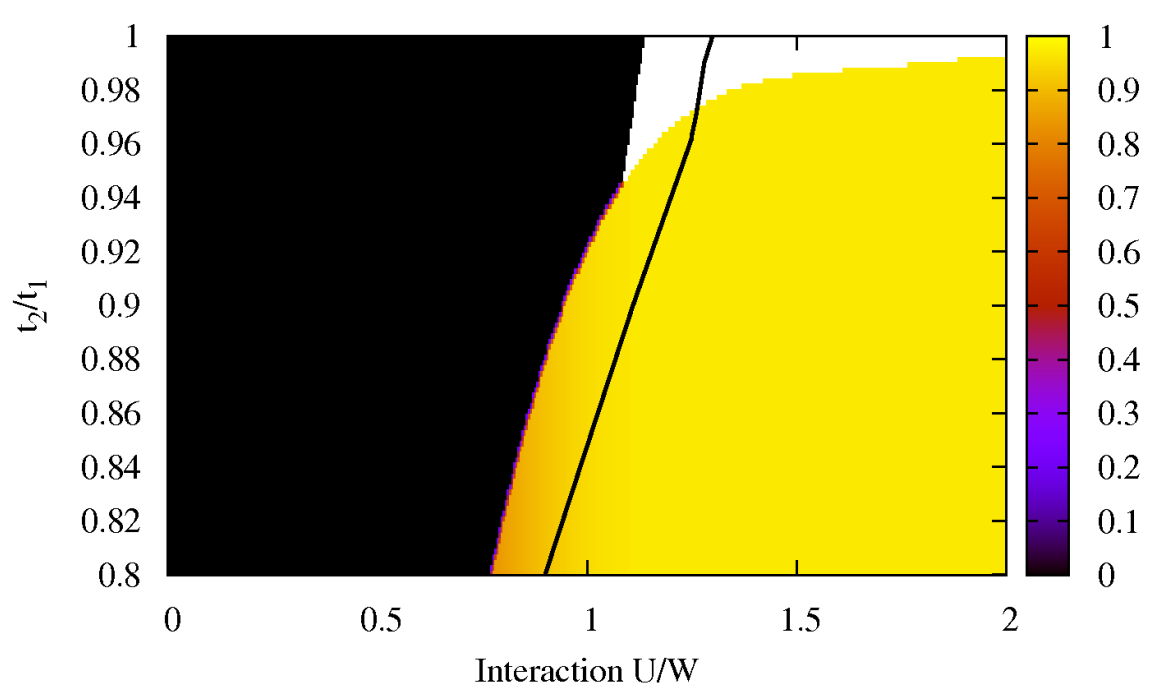

Figure 5.16: Ground state $(T=0)$ phase diagram for different strengths of frustration as function of the interaction. The black line within the magnetic phase signalizes the end of the hysteresis region for increasing and decreasing interaction. The phase boundaries of the incommensurate (IC) phase, white region, are only qualitative (explanation in text).

situation the ground state changes from a Néel-state for $J_{2}^{\star} / J_{1}^{\star}<1 / 2$ to a ground state with periodicity with longer than two sites for $J_{2}^{\star} / J_{1}^{\star}>1 / 2$. For the fully frustrated system $J_{1}^{\star}=J_{2}^{\star}$ the stable ground state configuration corresponds to $\theta=120^{\circ}$.

In DMFT calculations I can only allow for solutions commensurate with the lattice, for which I can derive a formula for the lattice Green's function. This however will possibly be inconsistent with the spin structure favored by the system. If, for example, I perform a calculation focusing on the ferromagnetic solution within a parameter regime, where the system wants to order antiferromagnetically, DMFT will not converge. To investigate spin density wave states with periodicity of more than two lattice sites, one has to set up the correct DMFT self-consistency equations respecting the lattice structure, as can be seen in chapter 5.2.3. While for a Bethe lattice with infinite coordination number and NN-hopping only it is straightforward to extend the DMFT equations to commensurate magnetic structures with periodicity of more than two lattice sites, I did not succeed in devising a scheme that allows for such calculations for systems with NNN-hopping. The NNN-hopping makes it impossible to uniquely identify the connectivity of the respective sublattices. A method proposed by M. Fleck et al. [156] for the two-dimensional cubic lattice is not applicable in this case.

I thus only allowed for paramagnetic, ferromagnetic and antiferromagnetic solu- 


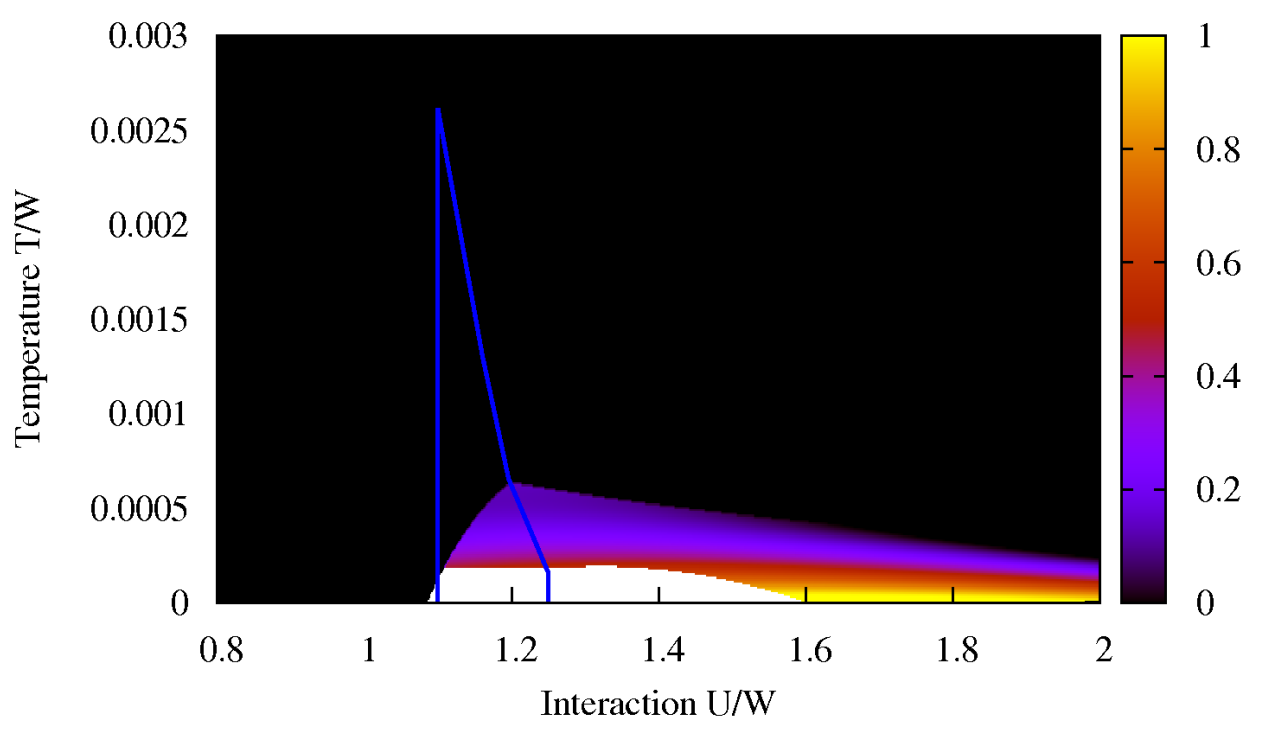

Figure 5.17: Magnetic phase diagram $T-U$ for $t_{2} / t_{1}=0.98$ for insulating starting medium including the PMIT as blue lines. The PMIT lies within the hysteresis region of the magnetic phase, but clearly outgrows it in temperature. The white region designates the IC-phase.

tions in my calculations. The resulting phase diagrams for $t_{2} \rightarrow t_{1}$ are shown in figures 5.16 and 5.17. Figure 5.16 displays the ground states for different grades of frustration and interaction strengths. For $t_{2} / t_{1}<0.95$ the phase diagram always has the same structure as for small and intermediate $t_{2}$. The critical interaction strength $U_{c}^{A F}$ necessary to stabilize the Néel-state increases and for all values above $U_{c}^{A F}$ I find an antiferromagnetic phase with a hysteresis region at the phase boundary. For $0.95<t_{2} / t_{1}<1$ the critical value $U_{c}^{A F}$ one needs to stabilize the Néel-state increases dramatically. For $t_{2}=t_{1}$ finally one does not find an antiferromagnetic Néel-state for any interaction strength $U$. The DMFT calculations however indicate that in this range of $t_{2} / t_{1}$ there actually does exists another magnetic phase. Namely, for sufficiently small temperatures one obtains a finite spin polarization in every DMFT iteration. However, the DMFT does not converge to a unique state as a function of the DMFT iteration (see also Fig. 5.4). In the phase diagrams in figures 5.16 and $5.17 \mathrm{I}$ have left this regime white designating an IC phase. In this parameter regime the Néel-state becomes unstable towards the behavior shown in figure 5.4. Here one can switch between a conventional Néel-state and the IC phase by only a small change of the interaction strength. Note, that the phase boundaries shown in the figure must be taken with some care as I cannot compare the energies of the Néel-state and this IC phase to properly determine the phase 
boundaries. As I observe precisely the same behavior for all investigated values $t_{2} / t_{1}=\{0.96,0.97,0.98,0.99,1.0\} \mathrm{I}$ am convinced that the ground state in this region is an incommensurate state, as to be expected from the results for classical spins. Similar observations also hold for finite temperatures as shown in figure 5.17, where the $T-U$ phase diagram for fixed $t_{2} / t_{1}=0.98$ is displayed. For increasing interactions and $T=0$ there is first a transition from a paramagnetic metal to the IC phase and for $U / W \approx 1.6$ from the IC phase to the Néel-state. For increasing temperature the IC phase eventually becomes unstable towards the Néel-state. In Fig. 5.17 one can also see the PMIT lines. The PMIT lies within the hysteresis region of the magnetic phases but clearly outgrows both magnetic phases. This is the scenario described in R. Zitzler et al. [147].

\subsubsection{Doped System}

As $t_{2}$ becomes finite the DOS becomes asymmetric and consequently the magnetic phase diagram becomes asymmetric with respect to half-filling, too. However, for sufficient small values of $t_{2}$, the magnetic phase diagram will still look very similar to that of the unfrustrated system shown in figure 5.2, with two notable exceptions: For the hole doped side of the phase diagram, the incommensurate magnetic phase sets in at smaller values of the interaction, while on the electron doped side it starts for larger values of the interaction. Thus, for electron doping the phase separation between the antiferromagnetic state at half-filling and the paramagnetic state at $n>1$ prevails for stronger interaction strengths. Already for $t_{2} / t_{1}=0.2$ I find no incommensurate phase on the electron doped side for $U / W<3$. As already stated above [136,147,165-167], in order to stabilize the antiferromagnetic phase for a finite NNN-hopping one needs a finite interaction strength $U_{c}^{A F}>0$.

For $1 / 4 t_{1}<t_{2} \leq t_{1}$ one obtains a strongly asymmetric DOS showing a squareroot singularity at the lower band edge. Here one can expect, and observe, a radically different phase diagram. Figures 5.18 and 5.19 show the antiferromagnetic phase diagrams for $t_{2} / t_{1}=0.6$. The first picture shows the magnetic phases for different chemical potentials $\mu / W$ in the same manner as in figure 5.2. In contrast to $t_{2}=0$, in the frustrated system the antiferromagnetic Néel-state can now be hole-doped at $T=0$. Therefore, I show the same phase diagram in figure 5.19 again, in which the occupation was substituted for the chemical potential. The boundaries of the IC-phase must be taken with care again, as this phase cannot be stabilized. It is interesting to note that the IC-phase is replaced by a doped antiferromagnetic phase. On the electron doped side of the phase diagram, I find neither an IC-phase nor the doped antiferromagnetic phase.

On further increasing the frustration to $t_{2} / t_{1}=0.8$, see figure 5.20 , the Néelstate does extend to large values of the doping $(\langle n\rangle=0.72)$, i.e. strong frustration seems to stabilize the Néel-state away from half-filling. The IC phase, on the other hand, completely vanished from the phase diagram. If one inspects figures 5.19 and 5.20 more closely, one sees that the antiferromagnetic state actually sets in away from half-filling for increasing interaction strength, i.e. 


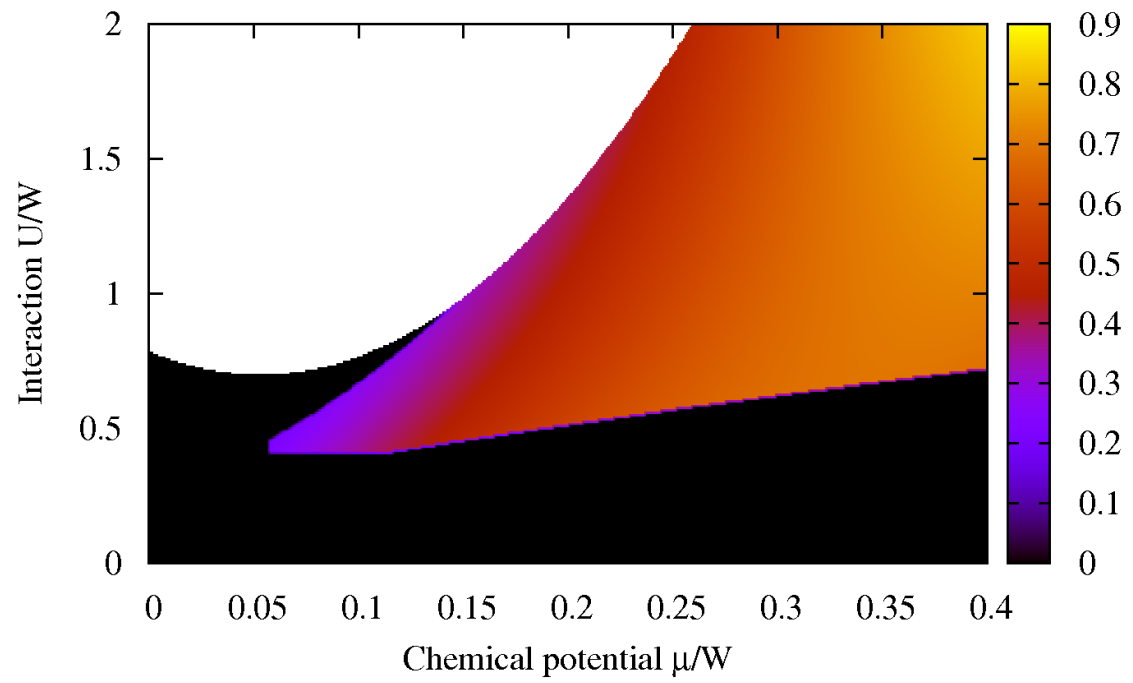

Figure 5.18: Antiferromagnetic ground state $(T=0)$ phase diagram $t_{2} / t_{1}=0.6$ for different chemical potentials $\mu$ and interaction strengths $U$. The color encodes the magnetization. The white region represents the IC phase.

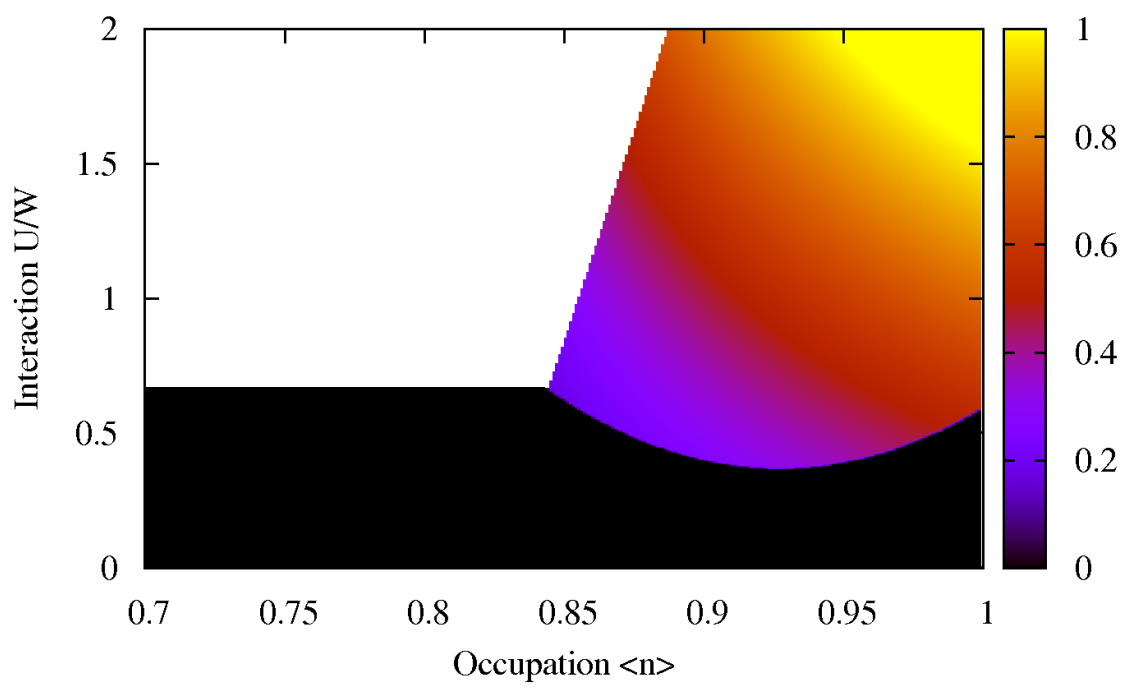

Figure 5.19: The same as in figure 5.18. As the antiferromagnetic phase exists now away from half-filling the chemical potential was substituted by the occupation $\langle n\rangle$. 


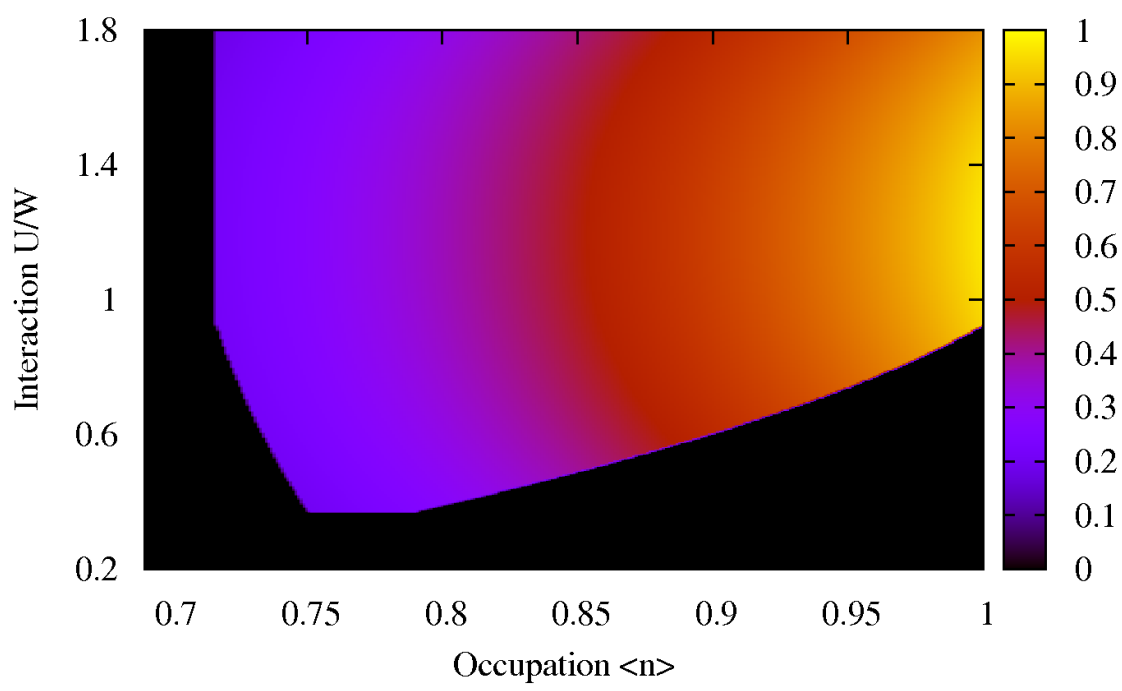

Figure 5.20: Antiferromagnetic ground state $(T=0)$ phase diagram for $t_{2} / t_{1}=0.8$. The color encodes the magnetization. The IC-phase present for $t_{2} / t_{1}=0.6$ has vanished.

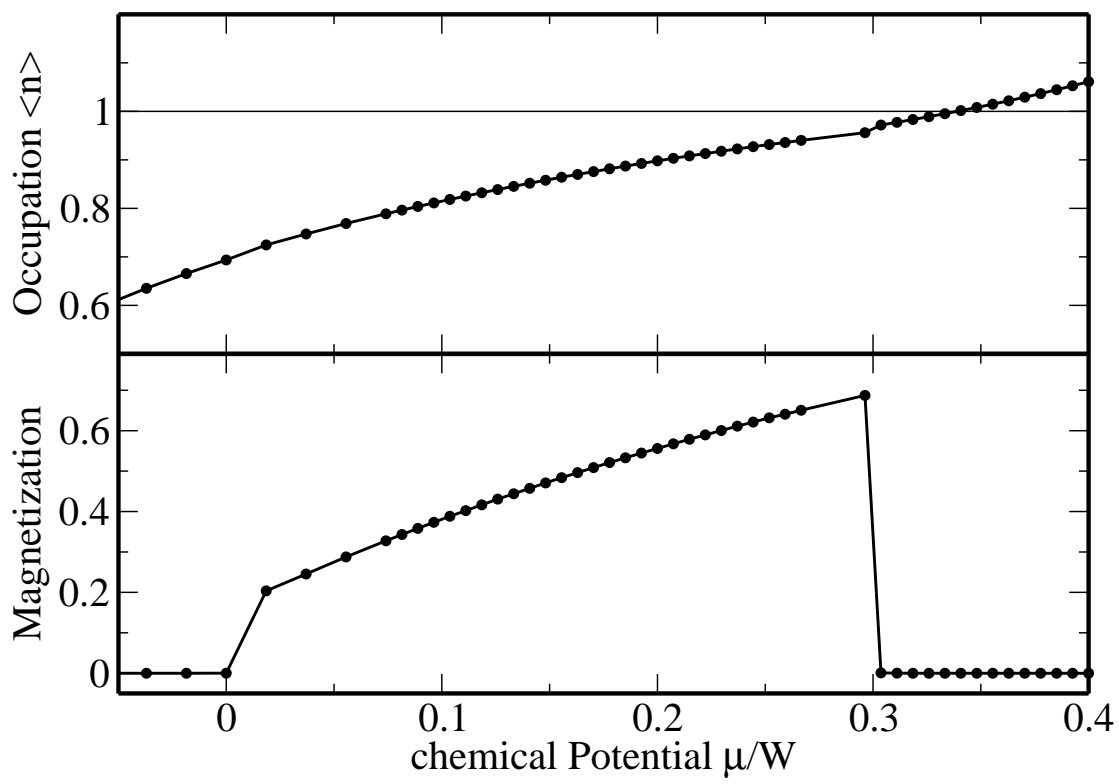

Figure 5.21: Ground state magnetization for $t_{2} / t_{1}=0.8$ and $U / W=0.75$. The upper panel shows the occupation and the lower panel shows the antiferromagnetic magnetization $n_{\uparrow}-n_{\downarrow}$. 


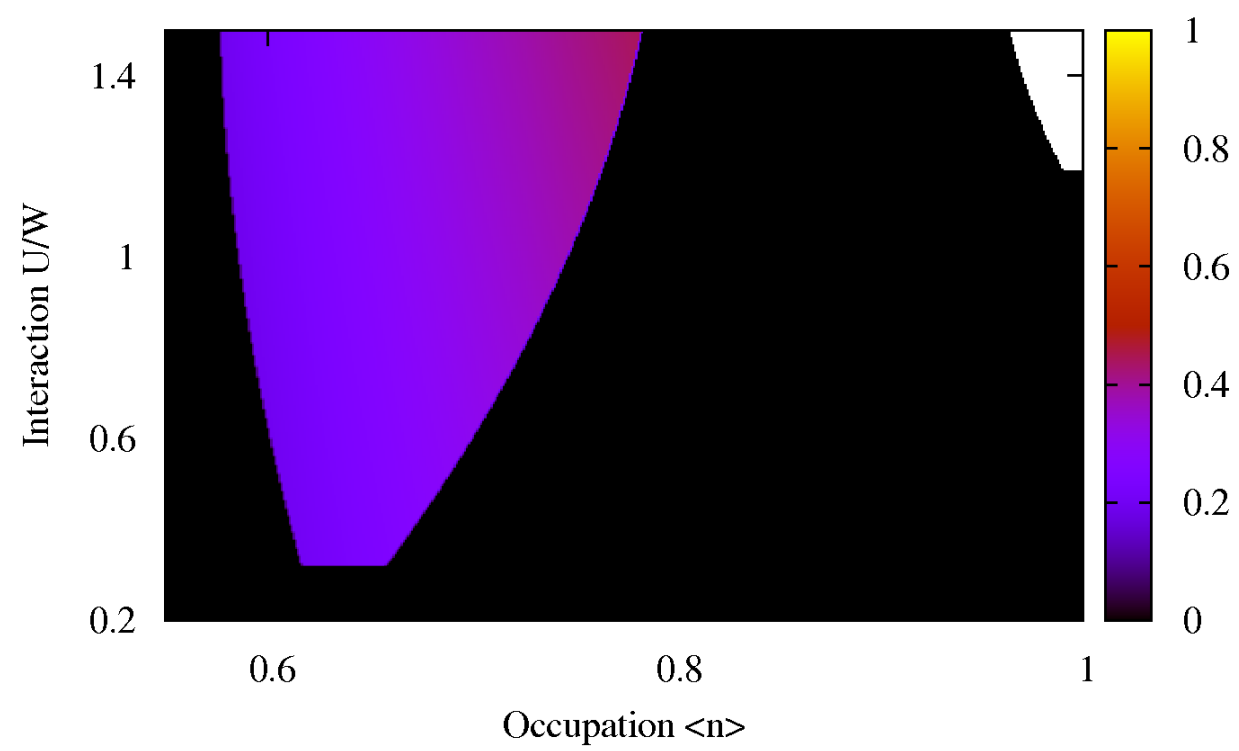

Figure 5.22: Antiferromagnetic ground state $(T=0)$ phase diagram for $t_{2} / t_{1}=1$, fully frustrated system. There is an antiferromagnetic phase, which exists only away from half-filling. For strong enough interaction there is a IC-phase at half-filling, white region.

$U_{C}^{A F}(n)$ has its minimum at $n<1$. At half-filling one finds for this values of interaction a paramagnetic metal. On the electron doped side, one only finds a paramagnetic state, which is still phase separated to the antiferromagnetic state at half-filling.

As discussed previously for half-filling, for large $t_{2} / t_{1}>0.96$ there appears a new phase which, motivated by a $120^{\circ}$-order expected for a classical spin system at this level of frustration, can be interpreted as such a spin density wave order. Figure 5.22 shows the phase diagram for $t_{2}=t_{1}$, i.e. a with respect to antiferromagnetic order fully frustrated spin system. The parameter region for large interaction left blank denotes precisely this spin density wave state, which also can be hole doped. What is most remarkable and rather mysterious, even for the fully frustrated system I find a stable antiferromagnetic Néel-state for fillings between $0.55<n<0.8$. To ensure that this result is not a numerical artifact, I performed several calculations at different temperatures and with different NRG parameters like discretization or states kept. However, for low enough temperatures I always find this antiferromagnetic island. I will come back to this point after discussing the ferromagnetic phase diagram.

\subsubsection{Ferromagnetism in the Frustrated system}

As already mentioned, while antiferromagnetism is the "natural" order occurring in single-band systems as studied here, ferromagnetic order is usually only obtained under more restrictive conditions. In this part I therefore want to 
focus on possible ferromagnetic solutions in the frustrated system.

One of the first heuristic treatments of metallic ferromagnetism was by E. Stoner [171]. He gave the criterion $U D_{F}>1$ for stabilizing ferromagnetism, where $U$ is the value of the on-site Coulomb interaction and $D_{F}$ is the value of the density of states at the Fermi level. Already in this criterion one sees that ferromagnetism is created by the interplay of the kinetic energy, characterized by $D_{F}$, and the Coulomb interaction, characterized by $U$. A rigorous result was obtained by Nagaoka [149], who proved the existence of ferromagnetism for $U=\infty$ and "one hole" for certain lattices.

In the beginning of the 1990's, A. Mielke and H. Tasaki proved the existence of ferromagnetism under certain conditions on the dispersion, known as "flat band ferromagnetism" $[172,173]$. Here the ferromagnetic ground state appears due to a dispersion-less (flat) lowest lying band. This flat band introduces a huge degeneracy of the ground state for $U=0$, which is lifted by the Coulomb interaction. A nice overview about this topic and other rigorous results for ferromagnetism can be found in the work of H. Tasaki [174].

Remembering the singularity in the DOS for $t_{2} / t_{1}>0.25$, the situation present in this system is very similar to the "flat band" scenario. Former studies for an asymmetric DOS [175-178] already showed the existence of ferromagnetism in such situations. Consequently, one has to expect ferromagnetism in this system, too. Indeed, Figure 5.23 shows the ferromagnetic polarization $p=\frac{n_{\uparrow}-n_{\downarrow}}{n_{\uparrow}+n_{\downarrow}}$, color encoded over the occupation $n=n_{\uparrow}+n_{\downarrow}$ and the interaction strength at low temperature $\left(T / W=2 \cdot 10^{-4}\right)$. The NNN-hopping for this system is $t_{2} / t_{1}=0.6$. The ferromagnetic state is fully polarized. One sees, that the singularity in the DOS alone cannot create ferromagnetism. Here one again needs a finite interaction strength of approximately $U / W \approx 0.3$, which however is a realistic number for transition metal compounds of both the $3 \mathrm{~d}$ and $4 \mathrm{~d}$ series. In figure 5.24 I depict the lower and upper critical occupation between which the ferromagnetic state is stable as function of the interaction strength. Below the lower critical occupation, the DMFT simulations do not converge independent of the interaction strength. I believe that this is a numerical problem due to the singularity in the DOS: If the Fermi level lies very close to the singularity, which is the case for $n \rightarrow 0$, the slope of the DOS at the Fermi level is very large. Small differences in the position of structures in the interacting Green's function will consequently have a great influence. However, I cannot rule out the possibility of the existence of another phase in this regime. The occupation number jumps in this region between almost zero and a larger value, and cannot be stabilized. The behavior can only be seen at low temperatures and for $t_{2} / t_{1}>0.25$, where the singularity in the DOS is sufficiently strong and not smeared out by temperature broadening.

At the upper critical occupation and low interaction strengths the system jumps from a fully polarized ferromagnet to a paramagnetic phase. For strong interaction the upper occupation is large enough such that the system directly changes from a ferromagnetic state into the incommensurate phase or the Néel-phase.

As I already noted, the "flat band" scenario indicates that the ferromagnetic state is intimately connected to the appearance of the van-Hove singularity at 


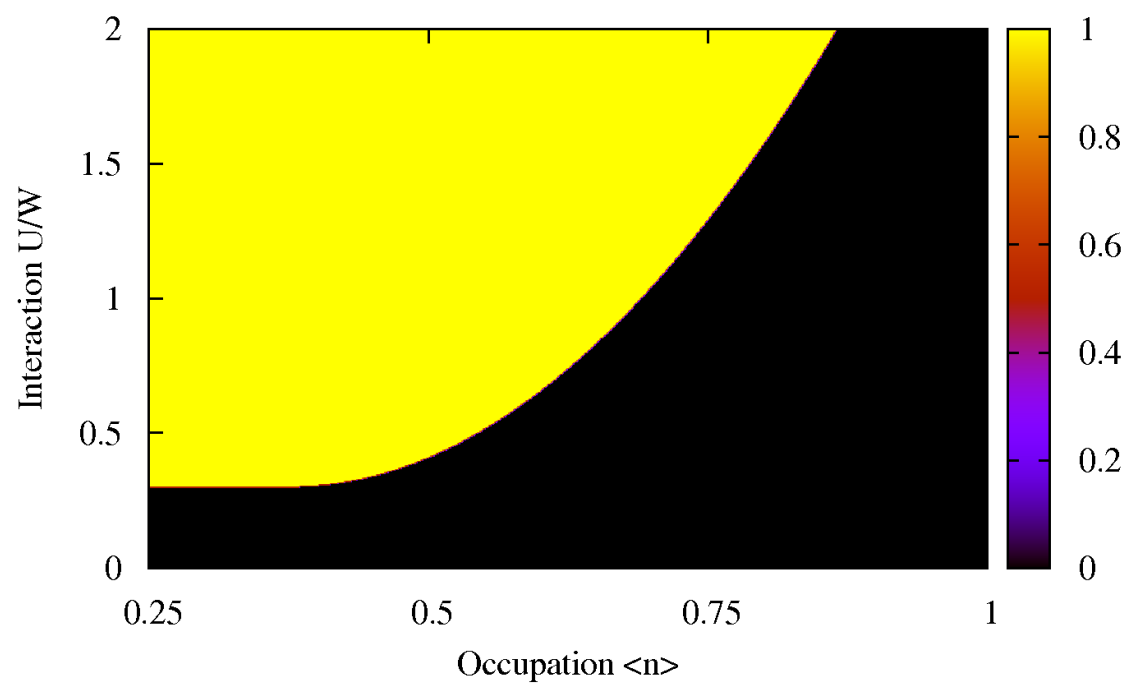

Figure 5.23: Ferromagnetic ground states $(T \approx 0)$ for $t_{2} / t_{1}=0.6$. Color coded is the polarization $p=\left(n_{\uparrow}-n_{\downarrow}\right) /\left(n_{\uparrow}+n_{\downarrow}\right)$.

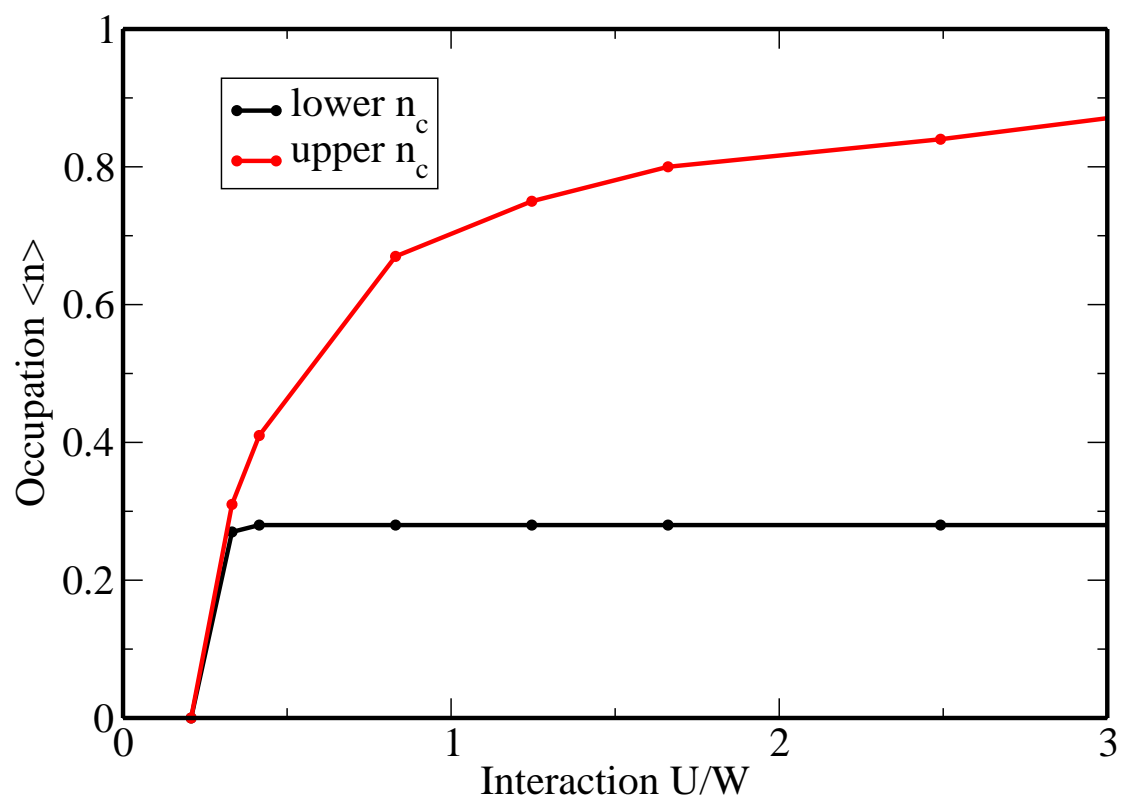

Figure 5.24: Upper and lower critical occupation between which the ferromagnetic phase exists for $t_{2} / t_{1}=0.6$ and $T=0$. 


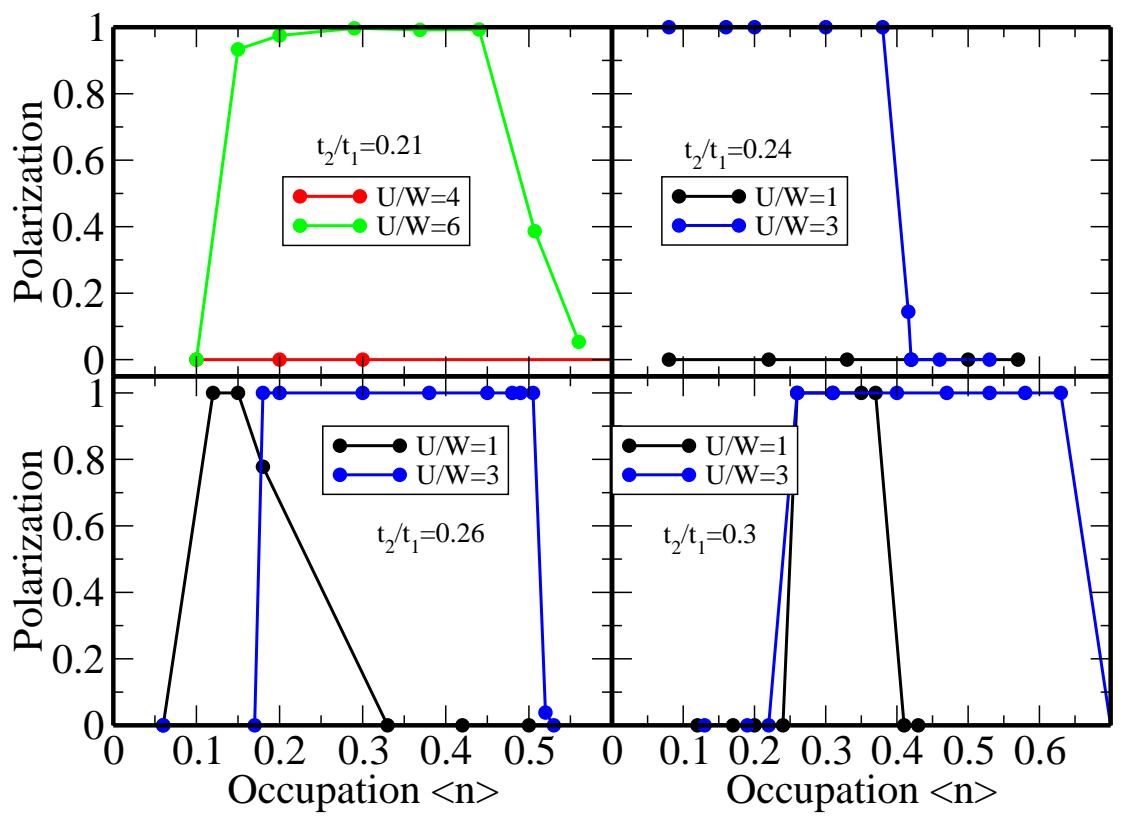

Figure 5.25: Ferromagnetic polarization for $T / W=2 \cdot 10^{-4}$ for $t_{2} / t_{1}$ as the singularity moves into the band. The upper panels show plots for a DOS without singularity. Note that with increasing $t_{2} / t_{1}$ the interaction one needs to stabilize the ferromagnet decreases.

the lower band edge. I will therefore look somewhat closer on the relation of the formation of a ferromagnetic state and the appearance of the singularity in the DOS. Figure 5.25 shows the polarization versus the occupation for different NNN-hopping $t_{2} / t_{1}$ and interaction strengths close to the appearance of the singularity in the DOS. The upper panels represent a situation where there is no singularity present in the DOS. The interaction needed to stabilize a ferromagnetic state in these systems without singularity is strongly increased. For the case of $t_{2} / t_{1}=0.2 \mathrm{I}$ found no ferromagnetic phase for interactions as strong as $U / W \approx 10$. As soon as $t_{2} / t_{1}>0.25$, the critical interaction strength lies below $U / W=1$. Increasing $N N N$-hopping $t_{2}$ as well as increasing the interaction strength favors the ferromagnetic state as it becomes more extended to larger occupations. In the DMFT/QMC study of J. Wahle et al. [175] a peak at the lower band edge was enough to stabilize a ferromagnetic phase at moderate interaction strengths. In my calculations the tendency towards ferromagnetism dramatically decreases for a DOS without singularity.

\subsubsection{Energies for the different magnetic states}

A careful look at the phase diagrams reveals, that there are parameter regions, where one seemingly can obtain both an antiferromagnetic as well as a ferromagnetic solution to the DMFT equations. This is rather unusual because conventionally DMFT will show oscillating behavior if one performs a ferromagnetic calculation in a regime with antiferromagnetic ground state and vice 
versa.

To decide which of the two solutions is the thermodynamical stable state, one has to compare their respective free energies. As the calculations were done practically at $T=0$, I calculate the energy of the system, given by

$$
\frac{\langle H\rangle}{N}=\frac{\left\langle H_{T}\right\rangle}{N}+\frac{U}{N} \sum_{i}\left\langle n_{i \uparrow} n_{i \downarrow}\right\rangle
$$

where $H_{T}$ is the kinetic energy and $N$ the number of sites. The interaction term $U$ is purely local and thus can be taken from the converged impurity calculation.

The kinetic energy, on the other hand, can be calculated from the expression

$$
\frac{\left\langle H_{T}\right\rangle}{N}=\int_{-\infty}^{\infty} d \theta \epsilon(\theta) \rho(\theta) \int_{-\infty}^{0} d \omega\left(-\frac{1}{\pi}\right) \mathfrak{I m} \frac{1}{\omega+\mu-\epsilon(\theta)-\Sigma(\omega+i \eta, \theta)},
$$

where $\Sigma(\zeta, \theta)$ is the lattice self-energy, $\theta$ a suitable variable to label the singleparticle energies on the lattice under consideration, and $\mu$ the chemical potential. Within DMFT, the lattice self-energy is approximated by a local selfenergy, i.e. one may set $\Sigma(\zeta, \theta)=\Sigma(\zeta)$. Furthermore, for the Bethe lattice with infinite coordination $\epsilon(\theta)=t_{1} \theta+t_{2}\left(\theta^{2}-1\right)$ and $\rho(\theta)=\frac{1}{2 \pi} \sqrt{4-\theta^{2}}$ holds. Substituting $\epsilon(\theta)$ by $\epsilon$ in the integral, the resulting DOS takes on the form given in chapter 2.5 .

Since the Néel-state is defined on an $A B$ lattice, one has to distinguish between the inter- and intra-sublattice hopping terms, and the formula for the kinetic energy takes on the form

$$
\begin{aligned}
\frac{\left\langle H_{T}\right\rangle}{N}=-\frac{1}{\pi} \mathfrak{I m} \int_{-\infty}^{\infty} d \theta \rho(\theta) \int_{-\infty}^{0} d \omega & \left(t_{1} \theta\left(G_{A B}(\omega+i \eta)+G_{B A}(\omega+i \eta)\right)\right. \\
& \left.+t_{2}\left(\theta^{2}-1\right)\left(G_{A A}(\omega+i \eta)+G_{B B}(\omega+i \eta)\right)\right)
\end{aligned}
$$

Note that with the definition of the matrix Green's function this formula can be put into the compact matrix form

$$
\begin{aligned}
\frac{\left\langle H_{T}\right\rangle}{N} & =-\frac{1}{\pi} \mathfrak{I m} \int_{-\infty}^{\infty} d \theta \epsilon(\theta) \rho(\theta) \int_{-\infty}^{0} d \omega \sum_{i j}[\underline{\underline{G}}(\omega+i \eta)]_{i j} \\
{[\underline{\underline{G}}(\omega+i \eta)]_{i j} } & :=\left(\begin{array}{cc}
\zeta_{\uparrow}-t_{2}\left(\theta^{2}-1\right) & -t_{1} \theta \\
-t_{1} \theta & \zeta_{\downarrow}-t_{2}\left(\theta^{2}-1\right)
\end{array}\right)_{i j}^{-1} \\
\zeta_{\sigma}(\omega) & :=\omega+\mu-\Sigma_{\sigma}(\omega+i \eta)
\end{aligned}
$$

The energies of the converged solutions for $t_{2}=t_{1}$ and $U / W=2.5$ can be seen in figure 5.26. The antiferromagnetic solution could be stabilized in this parameter region for occupations $0.55<n<0.8$. From figure 5.26 it becomes now clear that the ferromagnetic state has the lowest energy for $n<0.6$. For $0.6<n<0.75$ the antiferromagnetic state takes over as the ground state, but 


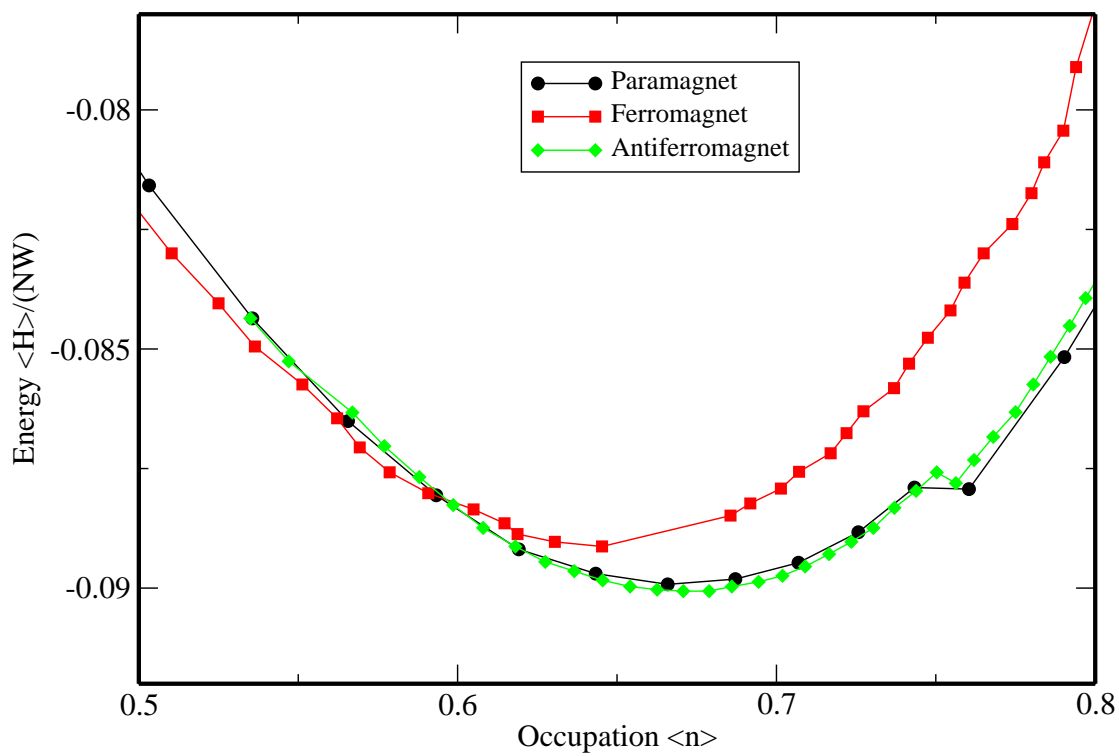

Figure 5.26: Ground state energies for paramagnetic, antiferromagnetic, and ferromagnetic states in the fully frustrated system for $U / W=2.5$. The energy was divided by the number of sites and the non-interacting bandwidth $W$.

is nearly degenerate with the paramagnetic state. For fillings larger than 0.8 no staggered magnetization can be stabilized any more.

Thus, the energy calculations reveal two things. Firstly, an antiferromagnetic Néel-state indeed seems to form away from half-filling in the fully frustrated system. Secondly, the energy differences are extremely small, in particular the antiferromagnet and paramagnet are de facto degenerate over the full parameter regime where the former exists.

To understand this at first rather irritating observation let me recall the wellknown fact that in strongly frustrated systems it is a common feature to have a large number of degenerate ground state configurations, which also can include magnetically ordered ones [174]. Thus, the degeneracy of the antiferromagnet and the paramagnet hints towards the possibility that there may exist a larger number of other magnetically ordered states in this parameter region. Unfortunately I am not able to search for and in particular stabilize those magnetic phases with the technique at hand.

\subsection{Summary}

In this chapter I analyzed the magnetic properties of an one-orbital Hubbard model within the DMFT for a Bethe lattice with infinite coordination. The unfrustrated Bethe lattice with semi-elliptic DOS shows antiferromagnetic behavior at $T=0$ only exactly at half-filling. Interestingly, the doping dependence of the antiferromagnetic phase changes from phase separation between the antiferromagnetic insulator at half-filling and the paramagnetic metal away from half-filling for weak interactions towards an inbetween lying incommensurate 
spin-density phase for strong interactions. This change happens exactly at the same interaction strength at which in the paramagnetic phase the metal insulator transition occurs. This means that one can see signs of the PMIT even in the antiferromagnetic phase, which actually completely covers the PMIT. One must note, however, that for finite $T$ this transition does not trace the PMIT, which maybe due to a stabilization of a doped antiferromagnetic phase, but creates room for discussion.

Furthermore, I proposed a way how to stabilize such spin-density waves for a Bethe lattice. Unfortunately, one has to test for each period separately and the calculation seem to be rather unstable.

For the frustrated Bethe lattice the magnetic phases change. The phase diagram becomes asymmetric towards half-filling. While for hole-doping an antiferromagnetic phase exists away from half-filling, for electron doping the phase separated region becomes more and more extended. For increasing frustration the antiferromagnetic doped state replaces finally the incommensurate spin density wave at the hole doped side of the phase diagram. Additionally, one now needs a finite interaction strength $U_{C}^{A F}>0$ for stabilizing the antiferromagnetic state. Interestingly, for the fully frustrated system, there still exists an antiferromagnetic Néel-phase away from half-filling. The Néel-state can be stabilized in the same parameter region as the ferromagnetic state. Comparing the energies of the possible states, there seems to be a transition from the ferromagnetic to the antiferromagnetic state. But the latter one is energetically almost degenerate to the paramagnetic state. Thus frustration seems to stabilize different states in this parameter region. It is an intriguing possibility, that there could be more degenerate magnetic states in this regime, stabilized due to the frustration. As I am, however, only able to look for homogeneous or Néel-states, this is only speculative, nevertheless motivating further studies of magnetic order in the single-band Hubbard model with different methods to solve the DMFT equations. However, for these studies the Bethe lattice may not be a suitable choice any more, as the definition of a wave vector $\vec{Q}$ to identify the various possible spin structures is not possible here. 
CHAPTER 6

\section{Two-Orbital Hubbard Model}

\subsection{Introduction}

Finally, I will present results for the two-orbital Hubbard model, describing two correlated degenerate bands. It was introduced in chapter 1.3, reading

$$
\begin{aligned}
& H=H_{T}+H_{U} \\
& H_{T}=\sum_{i j} \sum_{m=1}^{2} \sum_{\sigma} t_{i j, m} c_{i, m, \sigma}^{\dagger} c_{j, m, \sigma}, \\
& H_{U}=U \sum_{i} \sum_{m=1}^{2} n_{i, m, \uparrow} n_{i, m, \downarrow}+\left(U^{\prime}-\frac{1}{2} J\right) \sum_{i} \sum_{\substack{l, m=1 \\
l<m}}^{2} \sum_{\sigma, \sigma^{\prime}} n_{i, l, \sigma} n_{i, m, \sigma^{\prime}} \\
& -2 J \sum_{i} \sum_{l<m} \vec{S}_{i l} \cdot \vec{S}_{i m} .
\end{aligned}
$$

The operator $H_{T}$ represents the kinetic energy corresponding to the hopping of the electrons with amplitude $t_{i j}$. I assume throughout this chapter that there is only nearest neighbor hopping and that the hopping amplitudes are degenerate for both bands resulting in equal bandwidths $W=4 t$. The calculations were again performed for a Bethe lattice with semi-elliptic density of states. The interaction $H_{U}$ consists of three different terms: an intra-band density-density interaction with amplitude $U$, an inter-band density-density interaction with amplitude $U^{\prime}-\frac{J}{2}$, and a spin-spin interaction with amplitude $-2 J$, representing the ferromagnetic Hund's coupling for $J>0$. In the calculations shown in this chapter, I mainly concentrate on $U / W=4$, which is a good guess for transition metal oxides [9]. I will vary the Hund's coupling $J$ and the interorbital interaction $U^{\prime}$ and analyze their effects for different occupations of the system. 
The two-orbital Hubbard model is an appropriate model for describing the electronic correlations in manganites, as introduced in chapter 1.2.4. The phase diagram of $\mathrm{La}_{1-x} \mathrm{Ca}_{x} \mathrm{MnO}_{3}$ can be seen in figure 1.6. It shows a very rich phase diagram consisting of magnetic and orbitally ordered phases. Due to the cubic crystal symmetry the d-orbitals split into a threefold degenerate $t_{2 g^{-}}$and a twofold degenerate $e_{g}$-band, which for the coordination present in perovskites has the higher energy compared to the $t_{2 g}$-band. In $\mathrm{La}_{1-x} \mathrm{Ca}_{x} \mathrm{MnO}_{3}$ one has to distribute $4-x$ electrons per site to the d-states according to Hund's rules. Thus, the electronic situation in this compound can be modeled by a partially filled $e_{g}$-band below quarter-filling and a half-filled $t_{2 g}$-band, which couples via Hund's coupling ferromagnetically to the $e_{g}$-electrons. The hopping between the $t_{2 g}$-states is very small and thus the $t_{2 g}$-states are often modeled as localized $S=3 / 2$ spins. Besides this electronic part the lattice degrees of freedom and especially Jahn-Teller distortions are important to correctly describe the physics of manganites.

The aim of this chapter is to understand the role of the electronic degrees of freedom in the complex phase diagram of the manganites. In particular, to what extent the strongly correlated $e_{g}$-band is sufficient to reproduce the general features. Thus the investigations done here completely neglect the $t_{2 g}$-spin and the coupling to the lattice. Based on such an investigation, one can include additional interactions, like the coupling to the $t_{2 g}$-spin (or band) respectively the strong interaction with the lattice step by step, properly identifying for which particular features they are actually responsible. As I will discuss, already this simplified situation shows very complex ground state properties, involving the coupling of charge, spin and orbital degrees of freedom [179].

In the second section of this chapter, I will show calculations for two site clusters, in which the whole lattice consists of only two sites. The third section describes the magnetic ground state diagram of the two-orbital Hubbard model. In this section I focus on occupancies larger than one electron per lattice site. The final section concentrates on the quarter-filled system. Quarter-filling is a very special situation for the two-orbital Hubbard model. Exactly at quarter-filling, I can observe four different long range ordered phases. There is a competition of two different ferromagnetic states, an antiferromagnetic state, and a charge ordered state. Between the two ferromagnetic states one can find a metal insulator transition (MIT). These results are submitted for publication [180].

\subsection{Two Site Cluster}

Before studying the Hubbard model for a Bethe lattice within the DMFT, I will present some calculations for a two site cluster. This means that the whole lattice consists of only two sites, each built up from two orbitals. Altogether the system can be occupied by 8 electrons. But as the system is particle hole symmetric, I will only discuss the subsystems with 4 or less electrons. Even though one cannot expect to see the whole physics of the Hubbard model, one can get some insight into what one can expect.

The ground state of four electrons for a two site cluster, meaning half-filling, is 


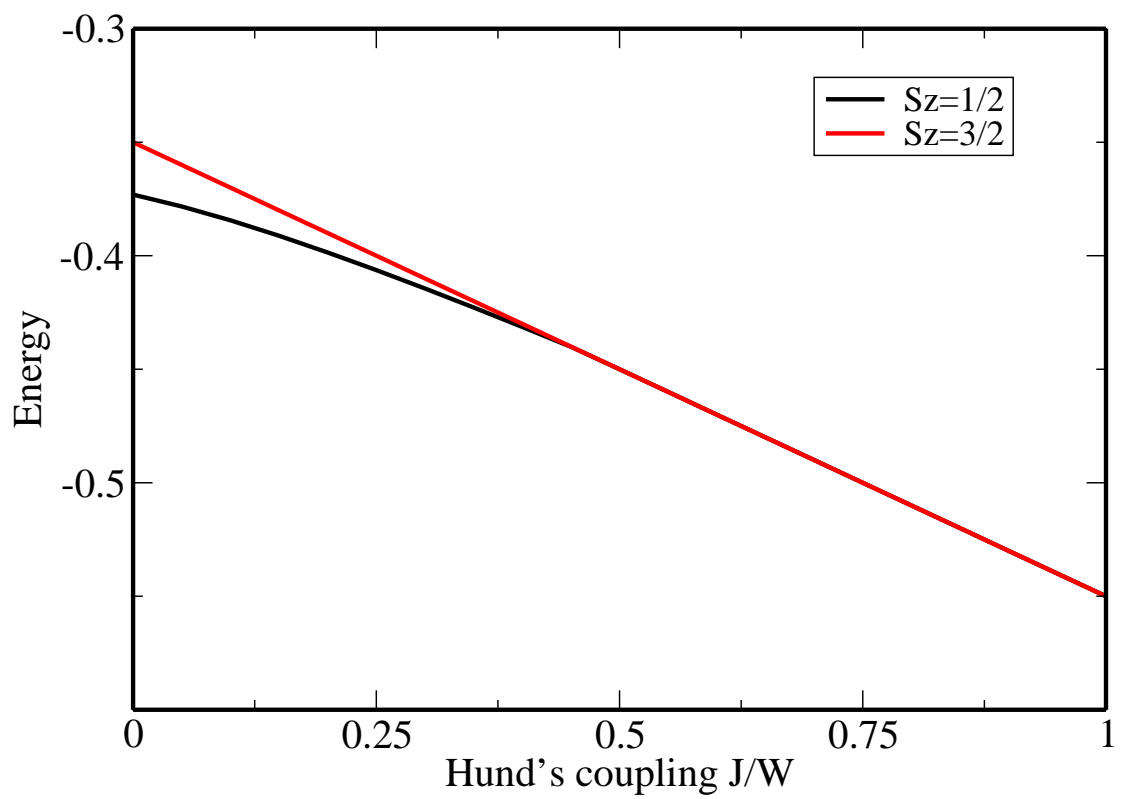

Figure 6.1: Exact diagonalization of a two site cluster with $t=-0.05, U=0.8$, $U^{\prime}=0.6, \mu=-0.3$ and 3 electrons in the system. For small Hund's coupling the ground state is a low spin configuration, while for large Hund's coupling the low and high spin configurations are degenerate. For scaling the x-axis, I have used the bandwidth $W=0.2$ of the free DOS in the next section.

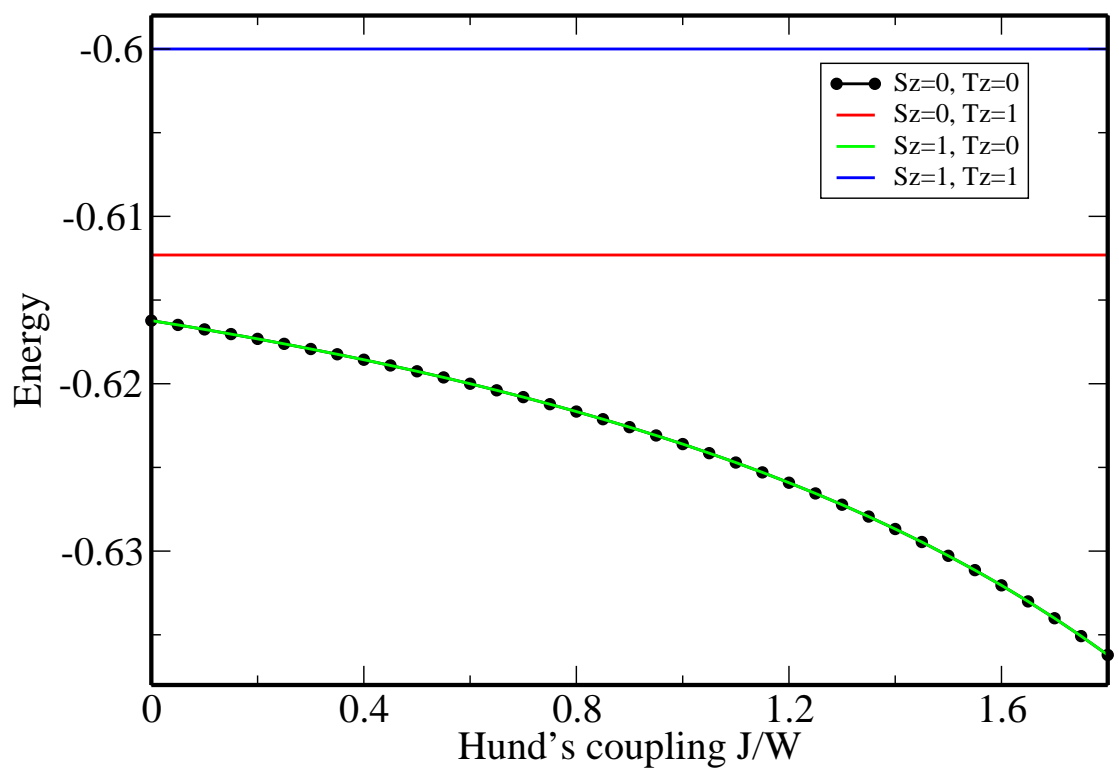

Figure 6.2: Exact diagonalization of a two site cluster with $t=-0.05, U=0.8$, $U^{\prime}=0.6, \mu=-0.3$ and 2 electrons in the system. The states with one electron in each orbital are degenerate ground states. I used again $\mathrm{W}=0.2$ for scaling the $\mathrm{x}$-axis. 
an antiferromagnetic state due to super exchange. Of course, one must be careful with the expression "antiferromagnetic" in a two site cluster. The ground state is a superposition of several possible states. The principal contributions come from the states, where one site consists of two up-electrons and the other of two down-electrons, and its interchanged configuration. If for a real lattice, like the Bethe lattice, long range order can persist, critically depends on the lattice and the temperature. For example, as seen in chapter 5 , frustration can prevent the system from forming an antiferromagnetic Néel-state. But in the calculations for a two site cluster, the states, from which an antiferromagnetic Néel-state would be built up, possess the lowest energy.

Figure 6.1 shows the ground state energies of the two site cluster with three electrons in the system. The interaction parameters are similar to the parameters, which are chosen in the next sections. The Hund's coupling is varied to see its influence on the ground state energy. The configuration with all three electrons in the same orbital has the highest energy, as one state must be double occupied and the local intra-band interaction $U$ is the largest interaction parameter. The remaining two configurations are such that two electrons are in the same orbital and the third electron occupies the other orbital. There is one ferromagnetic state with all spins aligned and therefore $S_{z}=3 / 2$, and one mixed state with $S_{z}=1 / 2$. Of course, the configurations with interchanged $S_{z}$-components, $S_{z}=-1 / 2$ and $S_{z}=-3 / 2$, exhibit the same energy. There is a level crossing in the quantum number subspace of $S_{z}= \pm 1 / 2$ at approximately $J / W=0.5$. For Hund's coupling $J$ below this value, the ground state is a doublet of $S_{z}= \pm 1 / 2$ states. Above this value the ground state is fourfold degenerate consisting of one state from the $S_{z}= \pm 1 / 2$ subspace and one state from the $S_{z}= \pm 3 / 2$ subspace each. The states from the $S_{z}= \pm 3 / 2$ subspace represent the possibility of a ferromagnetic state in an extended system. This shows the importance of the Hund's coupling $J$ for ferromagnetism in this system. One will see in the next section, that also in an extended system there is a ground state transition towards a ferromagnetic phase for occupation $\langle n\rangle \approx 1.5$ when increasing $J$.

In figure 6.2 one can see the ground state energies for the case, when there are altogether two electrons on both sites, representing quarter-filling. There are four quantum number subspaces under consideration. Quantum number $S_{z}=0$ corresponds to the situation of one up electron and one down-electron, while for $S_{z}=1$ both electrons possess spin component $S_{z}=1 / 2$. The subspace with both electrons having $S_{z}=-1 / 2$ is degenerate to the last one. The same discrimination can be done for the orbitals. As the pair hopping term was neglected in this work, one can introduce a conserved orbital quantum number $T_{z} \cdot T_{z}=0$ means, that one electron is in the orbital, represented by $m=1$ in the Hamiltonian, and the other electron occupying $m=2$. For $T_{z}=1$ both electrons occupy the same orbital. The ground state energies in figure 6.2 show that there is a degenerate ground state with $T_{z}=0$. The configuration corresponds to a triplet state in the spin space, but the $S_{z}=-1$ state is not shown here. In an extended bipartite lattice this configuration can result in a Néel-state for the orbital occupation: On the A-sites of the lattice the electrons sit on the $m=1$ orbital, while on the B-sites the electrons sit on $m=2$. I will 


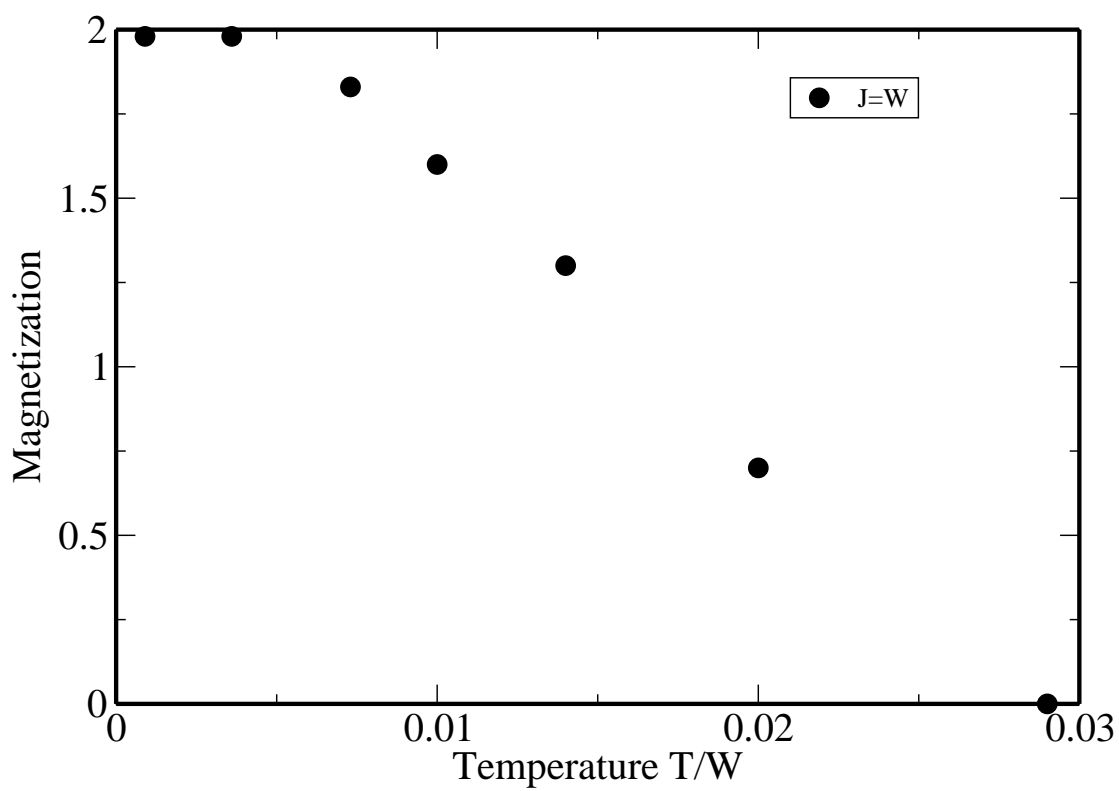

Figure 6.3: Antiferromagnetic magnetization $m=n_{\uparrow}-n_{\downarrow}$ versus the temperature for $U / W=4, J / W=1$, and $U^{\prime} / W=2$ at half-filling. The transition does only change a little upon varying $U^{\prime}$ or $J$.

show results on such states later in this chapter about quarter-filling.

Calculations with altogether only one electron are not shown here, as all interaction terms vanish. Thus all states possess the same energy of $E=-\mu+t$.

\subsection{Magnetic Phase Diagram}

For an extended system like the Hubbard model for a Bethe lattice the situation is different from the two site cluster. Even though the ground state of the two site cluster consists of aligned spins, it is a priori not clear, if the ground state is ferromagnetical for an infinite lattice. I will here only present results for the semi-elliptic free density of states (DOS) of the infinite dimensional Bethe lattice, corresponding to a bipartite situation. All results are obtained within the DMFT with the NRG as impurity solver. The two-orbital model is an extreme case for using the NRG. The calculations were performed parallelized on eight-core multiprocessor architectures using up to 15 GB memory. For all calculation the NRG parameters $\Lambda=2.0$ and $N_{K}=4000-5000$ were used.

As the intra-orbital density-density interaction $U$ is usually the strongest interaction present, the system is a good candidate for super exchange at halffilling [51] (chapter 1.4). And indeed, the system forms an antiferromagnetic insulator at half-filling. The temperature dependence of the antiferromagnetic magnetization $m=n_{\uparrow}-n_{\downarrow}$ is shown in figure 6.3. With increasing temperature the magnetization vanishes smoothly, signaling a second order phase transition. The transition temperature and the characteristics of the transition mainly depend on the intra-orbital interaction $U$. As in the one-orbital 

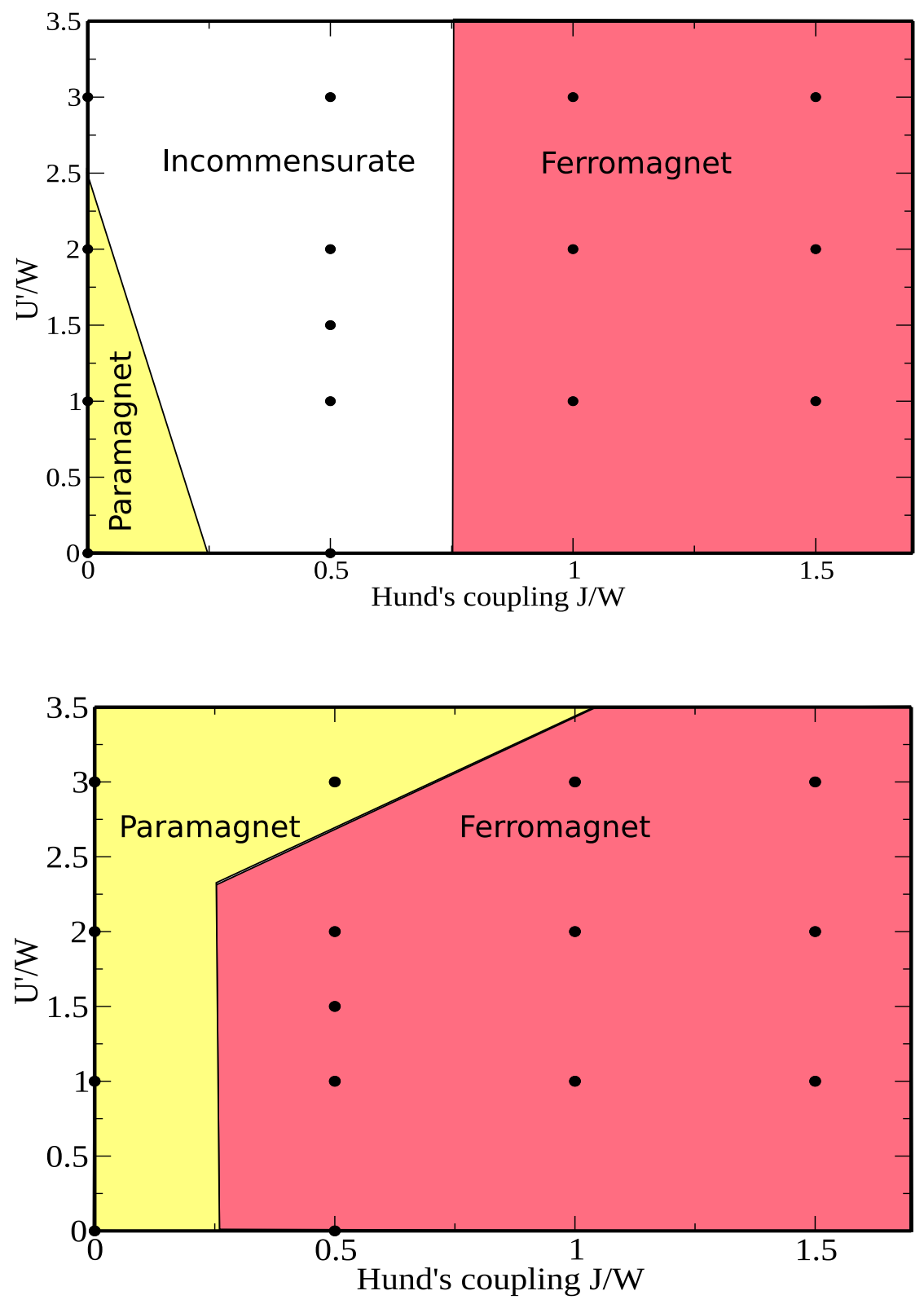

Figure 6.4: Magnetic ground state phase diagram, $U / W=4, T / W=2 \cdot 10^{-4}$, for $\langle n\rangle=1.5$ (upper panel) and $\langle n\rangle=1.2$ (lower panel). For stabilizing a ferromagnetic phase, one needs a finite Hund's coupling $J>0$. For $\langle n\rangle=1.5$ the incommensurate phase extending from half-filling, is still present. This phase has vanished for $\langle n\rangle=$ 1.2. The points denote the parameters, at which calculations were done. The phase boundaries are only schematic. 
Hubbard model for the unfrustrated Bethe lattice, this antiferromagnetic Néelstate cannot be doped. The antiferromagnetic state becomes unstable towards an incommensurate spin density wave, when changing the chemical potential $\mu$. This was already shown for such strong interaction strengths in chapter 5 for the one-orbital Hubbard model.

The phase diagram of the one-orbital Hubbard model with semi-elliptic DOS, corresponding to a Bethe lattice without frustration, does not show any other ordered phases. In contrast, there are parameter regions in the two-orbital Hubbard model, which are dominated by double exchange (chapter 1.4), showing large regions of ferromagnetism [33,181-187]. I have shown in the last section for three electrons in a two site cluster, that there is a ground state transition when increasing $J$ and that the state with parallel aligned electrons becomes the ground state for large enough ferromagnetic Hund's coupling $J$. The same holds true for the Bethe lattice. For $\langle n\rangle=1.5$, there is a transition from the incommensurate phase to a ferromagnetic phase for increasing Hund's coupling, see figure 6.4. The incommensurate phase shows the same non-convergent behavior in the DMFT as described in the last chapter. This phase extends up to half-filling, where it is displaced by the antiferromagnetic Néel-state. For $\langle n\rangle=1.2$ the incommensurate phase has completely vanished. This phase is most probably due to antiferromagnetic tendencies at half-filling, which are in the doped system incommensurable with the filling of the lattice and vanish when doping too far away from half-filling. The ferromagnetic state, on the other hand, is stabilized by the Hund's coupling $J$. Interestingly, strong Hund's coupling alone is not enough. For $U / W=2$ the ferromagnetic tendency in the parameter region for $\langle n\rangle=1.2-1.5$ decreases rapidly. For $U / W=2$ it is only possible to stabilize a ferromagnetic phase for $J / W=U^{\prime} / W=2$, which, however, is a questionable large Hund's coupling $J$ compared to $U$. For lower values of the Hund's coupling and $U / W=2$ the phase diagram for $\langle n\rangle=1.0-1.5$ is dominated by a paramagnetic metallic phase. In contrast, for $U / W=4$ the ferromagnetic phase covers most of the magnetic phase diagram for filling $\langle n\rangle=1.2$. Only for low Hund's coupling the paramagnetic phase is the only stable one. The ferromagnetic phase diagrams for the whole occupation and temperature range and $U / W=4, J / W=1, U^{\prime}=2$ (upper panel) and $U / W=4, J / W=1.5, U^{\prime}=1$ (lower panel) can be seen in figure 6.5. In both diagrams the ferromagnetic polarization $p=\frac{n_{\uparrow}-n_{\downarrow}}{n_{\uparrow}+n_{\downarrow}}$ is color coded. The white region denotes the already mentioned incommensurate phase. For better comparison, the same division of axis was chosen in both diagrams. Therefore, one can see how the ferromagnetic phase becomes more extended to larger values of hole doping when increasing the Hund's coupling. For $J / W \geq 1.5$ the ferromagnetic phase extends to fillings less than quarter-filling $\langle n\rangle=1$. This will become important in the next section. So far, the magnetic phase diagram of the two-orbital Hubbard model consists of an antiferromagnetic phase at halffilling, which becomes unstable towards an incommensurate spin density wave when doping, and eventually changes to a ferromagnetic state at occupation $\langle n\rangle \approx 1.5$ if the Hund's coupling $J$ is large enough. 

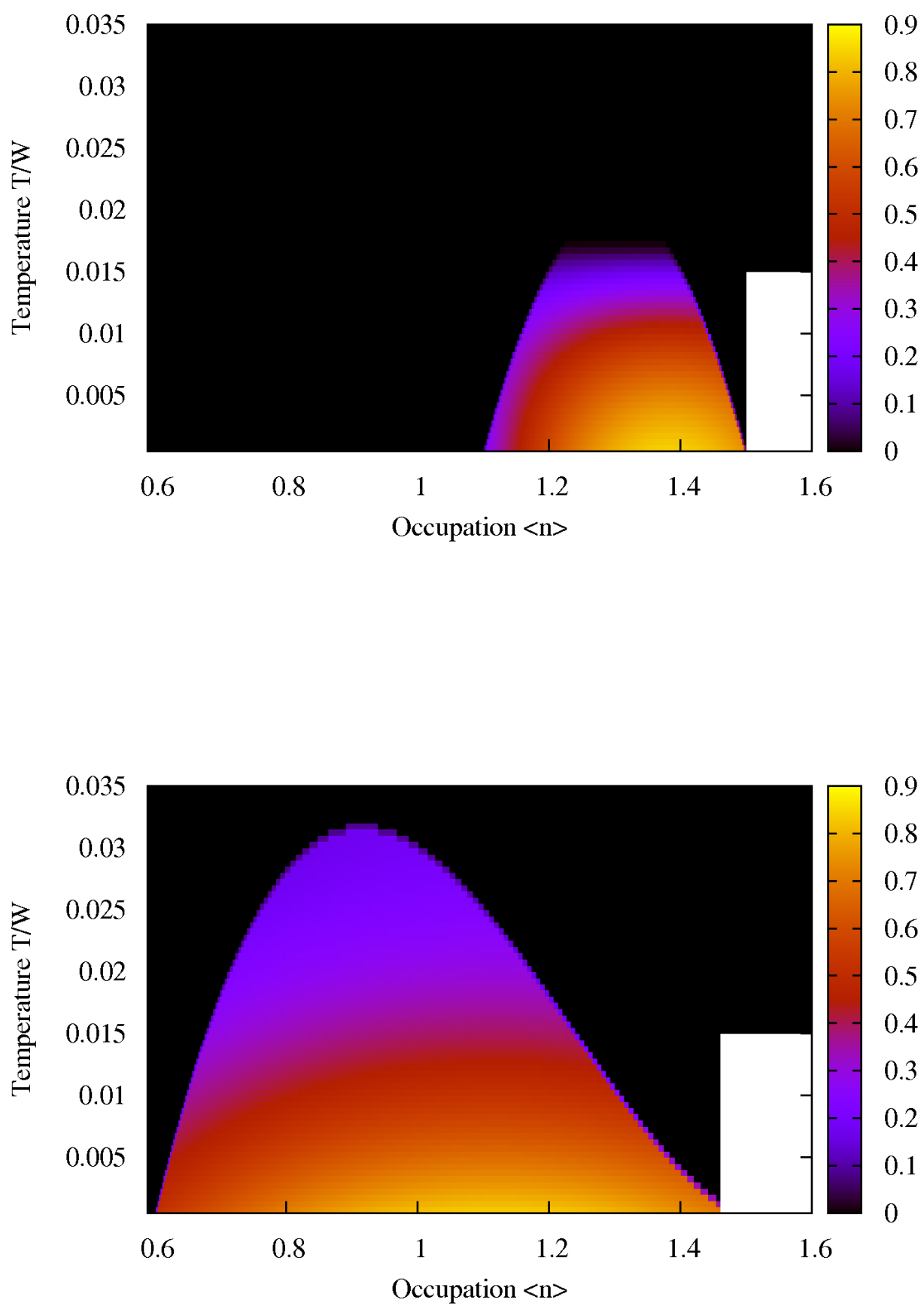

Figure 6.5: Magnetic ground state phase diagram for $U / W=4, J / W=1, U^{\prime} / W=2$ (upper panel) and $U / W=4, J / W=1.5, U^{\prime} / W=1$ (lower panel). The ferromagnetic polarization $p=\frac{n_{\uparrow}-n_{\downarrow}}{n_{\uparrow}+n_{\downarrow}}$ is color coded. The white region corresponds to the incommensurate phase. In this parameter region the DMFT calculations did not converge. Both phase diagrams were created by fitting of approximately 30 points each. Notice that for both diagrams the same division of axis was chosen. 


\subsection{Quarter-Filling}

\subsubsection{Ferromagnetic Metal Insulator Transition}

Quarter-filling, like half-filling, represents a very special point for the two-orbital Hubbard model. In a classical picture there is one electron per site, which can choose between two orbitals. This picture already makes clear that orbital degeneracy and fluctuations play an important role for quarter-filling. This was confirmed by the two site cluster calculation of the last section. In figure 6.6, I show the ferromagnetic ground state phase diagram for $\langle n\rangle=1$ as function of $U^{\prime}$ and $J$. The intra-orbital interaction was $U / W=4$. As noted before, for strong Hund's coupling one obtains the orbitally homogeneous ferromagnetic phase discussed in figure 6.5. However, for large enough repulsive inter-orbital density-density interaction $U^{\prime}$, I observe that the ferromagnetic spin alignment is accompanied by an antiferro-orbital order of the conventional Néel-type [182, 183,187-191]. The phase diagram at $\langle n\rangle=1$ is mainly covered by ferromagnetic phases. Only for small values of the Hund's coupling a paramagnetic phase can be found as a ground state. Figure 6.7 shows the spectral functions of both

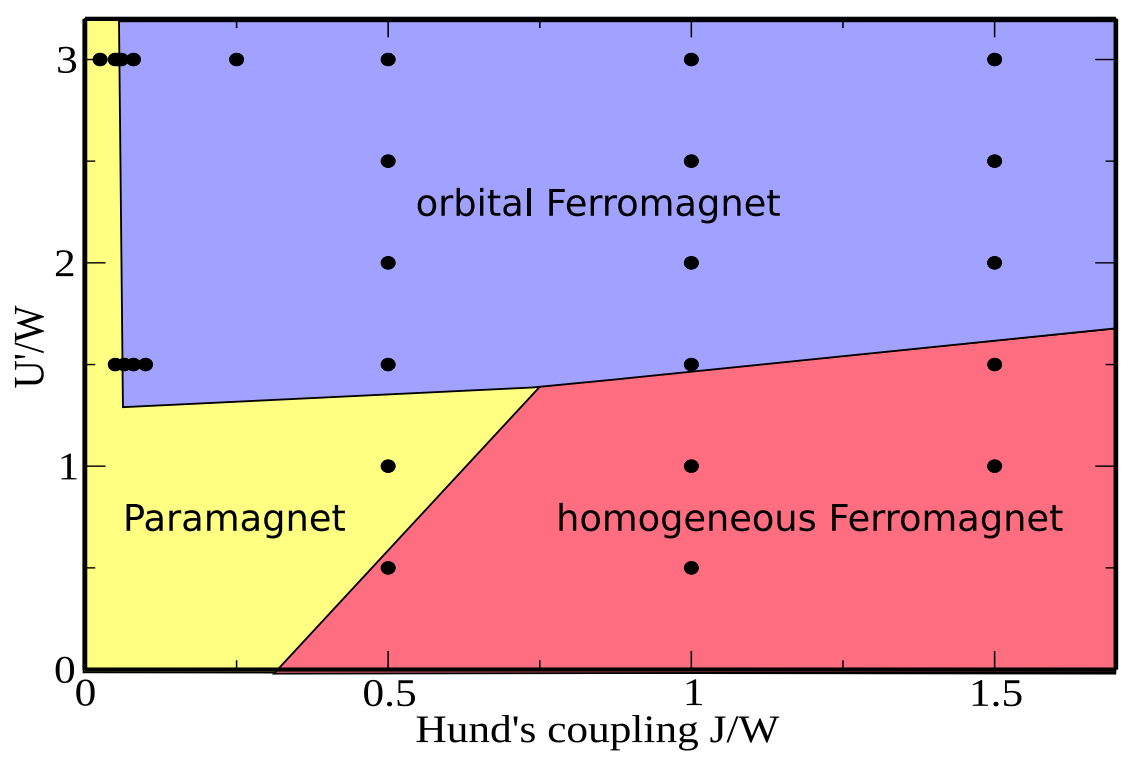

Figure 6.6: Ferromagnetic phase diagram for $U / W=4$ and $\langle n\rangle=1$. The calculations were done at the parameters denoted by the black points. Therefore the phase boundaries are only schematic.

ferromagnetic states. The left (right) panel shows the configuration and the spectral function of the homogeneous (orbitally ordered) phase. These results were obtained at $T / W=2 \cdot 10^{-4}$. The Fermi energy corresponds to $\omega=0$. Thus one can observe a dramatic difference in the behavior of both states at the Fermi energy. The majority spin of the homogeneous ferromagnetic state has large spectral weight at the Fermi level, showing metallic behavior. In contrast, the orbitally ordered ferromagnetic state possesses a gap at the Fermi energy. There is no spectral weight. Thus the orbitally ordered ferromagnet is an insulator. 


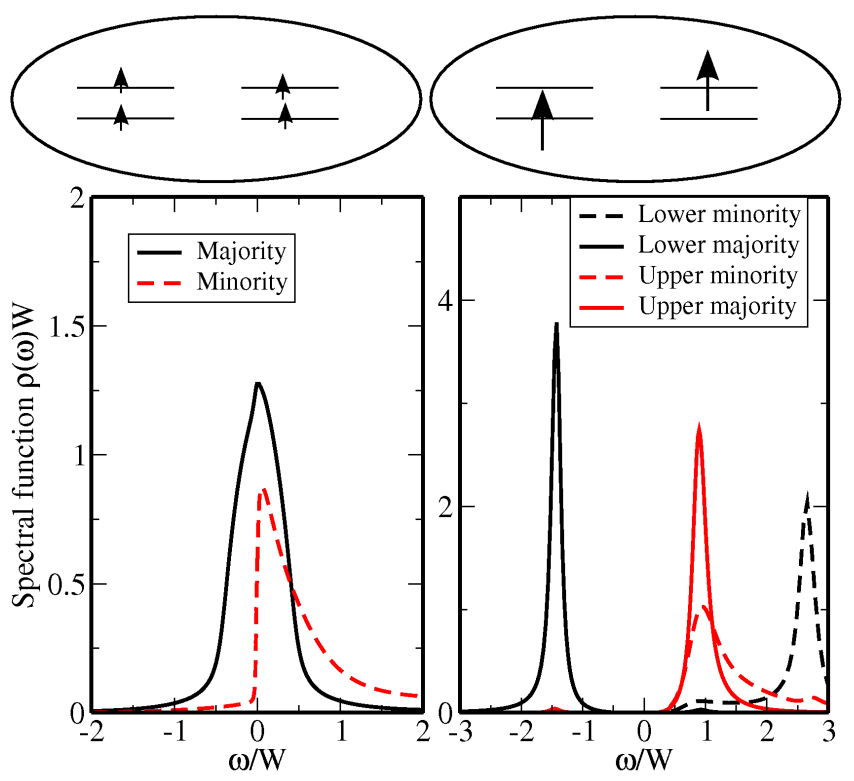

Figure 6.7: Spectral functions and schematic occupation of the orbitals within both ferromagnetic phases. Left: Homogeneous ferromagnetic state at $J / W=1.5$ and $U^{\prime} / W=1$. Right: Orbitally ordered ferromagnetic state at $J / W=0.5$ and $U^{\prime} / W=3$. Note the different axis divisions.

The evolution of the spectral function of the orbitally ordered ferromagnetic state and the gap size can be seen in figure 6.8. One can observe how the gap size linearly shrinks and finally closes at $U^{\prime} / W \approx 1.4$. Thus, by varying Hund's coupling $J$ or $U^{\prime}$ one can observe a metal insulator transition (MIT) between two almost fully polarized ferromagnetic phases. Note that this metal insulator transition is very different from the usual paramagnetic one, which appears in the Hubbard model as function of $U$ in the paramagnetic state. The observed MIT within the ferromagnetic phases, however, is due to a strong interorbital density-density interaction, which is responsible for driving the orbital ordering. The usual intra-orbital Hubbard interaction $U$ plays a minor role in this transition. There is a first order transition between the orbitally ordered ferromagnetic state and the homogeneous one: both the magnetic and orbital polarization show jumps when crossing the phase boundary, which can be seen in figure 6.9. Note that both states are nearly fully polarized; the orbitally ordered ferromagnetic state has $p=\frac{n_{\uparrow}-n_{\downarrow}}{n_{\uparrow}+n_{\downarrow}}=1$, while the homogeneous has $p=0.82$. This means that the jump in the magnetization is comparatively small. A more important aspect is that the orbitally ordered ferromagnetic phase is an insulator, while the homogeneous one is a metal.

Both states show very different dependence on the chemical potential. For the homogeneous ferromagnetic phase I already showed, when discussing figure 6.5 , that its filling can be varied smoothly. The dependence on the chemical potential of the orbitally ordered ferromagnet on the other hand is completely 

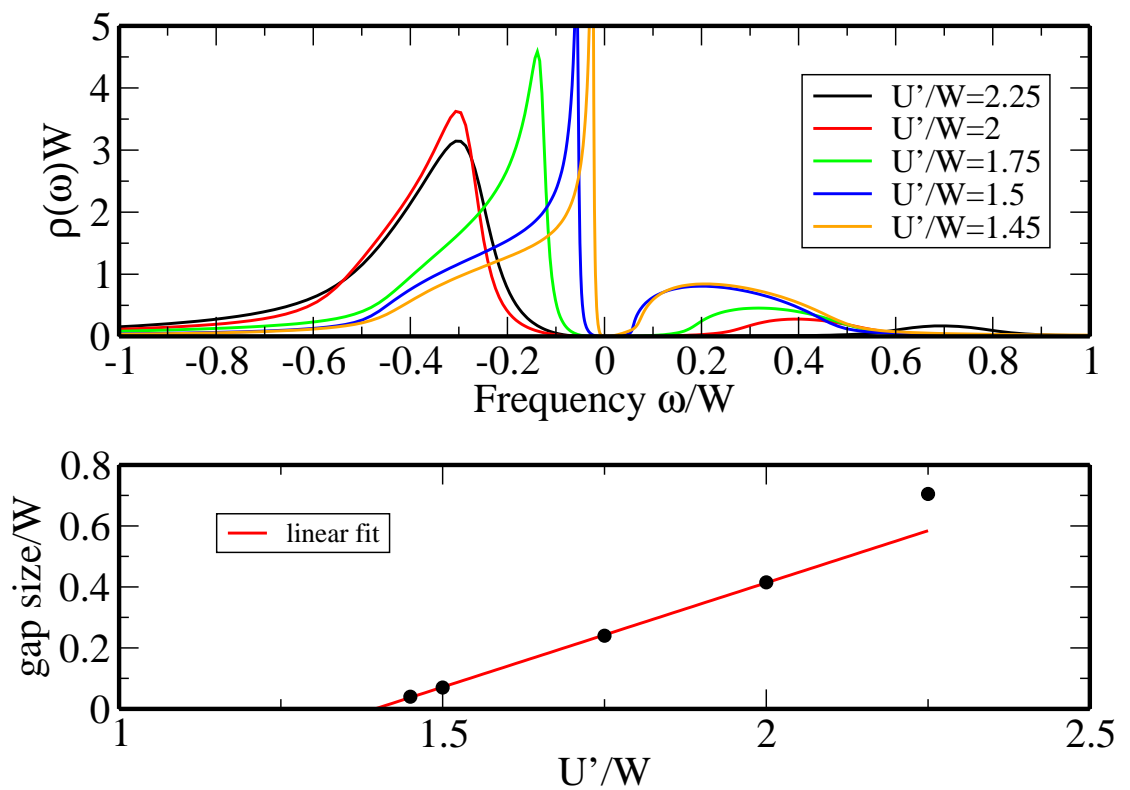

Figure 6.8: Top: Evolution of the spectral function of the orbitally ordered ferromagnetic state for $U / W=4$ and $J / W=1$. Bottom: Size of the gap extracted from the spectral functions as function of $U^{\prime}$. The red line corresponds to a linear fit.

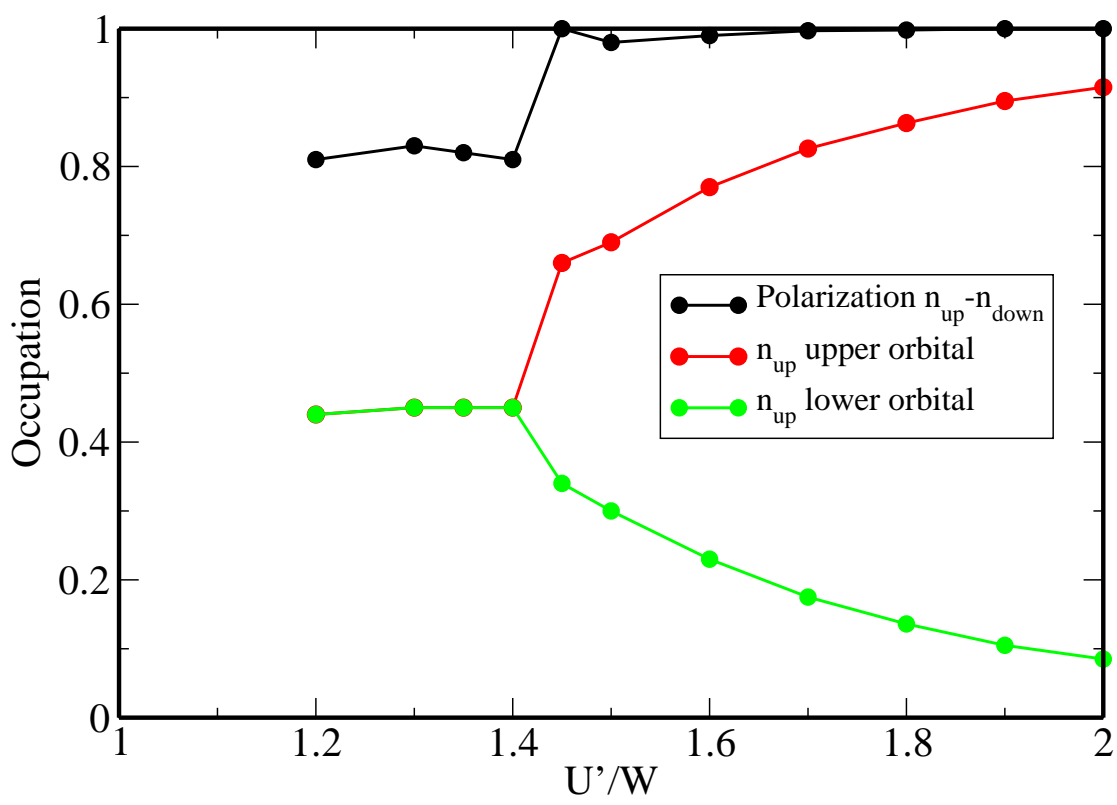

Figure 6.9: Dependencies of the occupations on $U^{\prime}$ near the metal insulator transition for $U / W=4$ and $J / W=1$. The black points denote the polarization of the ferromagnetic state. The red and green points show the occupation for the majority spin for the upper and lower orbital. 


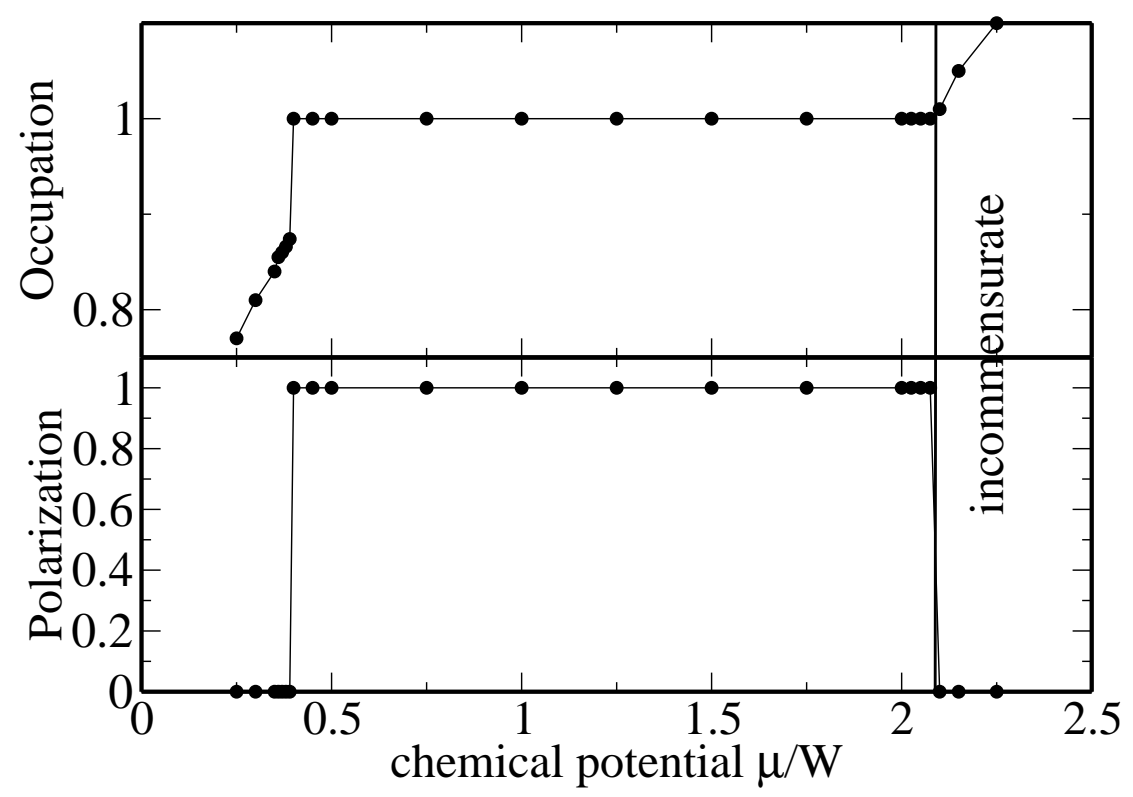

Figure 6.10: Dependence of the orbitally ordered ferromagnetic state for $J / W=0.5$ and $U^{\prime} / W=3$ upon changing the chemical potential $\mu$. The upper (lower) panel shows the occupation (polarization) of the system.

different and can be seen in figure 6.10. The plot shows that for a critical chemical potential the filling of the system jumps from approximately $\langle n\rangle=0.88$ to quarter-filling $\langle n\rangle=1$. At the same critical chemical potential also the polarization jumps from a non-polarized state to a nearly fully polarized. In other words, there is a first order transition between a paramagnetic phase with filling less than one and an orbitally ordered ferromagnetic state at quarterfilling. Consequently the electronic system shows phase separation between these states and the precise physics will depend on additional interactions, like long-range Coulomb interaction, or additional degrees of freedom like the lattice. For electron doping this orbitally ordered ferromagnet becomes unstable towards an incommensurate spin density wave. Unfortunately, it is very hard to stabilize such a spin density wave in our calculations, so that I am not able to study the exact nature and energetic stability of this phase.

With respect to effects like the colossal magneto resistance effect in manganites, introduced in chapter 1.2.4, it should be very interesting to look at the temperature dependence of the orbitally ordered ferromagnetic state. In manganites a ferromagnetic metal becomes unstable towards a paramagnetic insulating state when increasing the temperature. The transition temperature can be tuned by applying a magnetic field, which explains the large resistance change when varying the magnetic field. The metal insulator transition observed in the twoorbital Hubbard model provides the opportunity to see similar effects. The question is, whether this MIT can be triggered by applying a magnetic field or changing the temperature. Figure 6.11 shows the temperature dependence of a state deep within the orbitally ordered ferromagnetic phase. The ground state of 


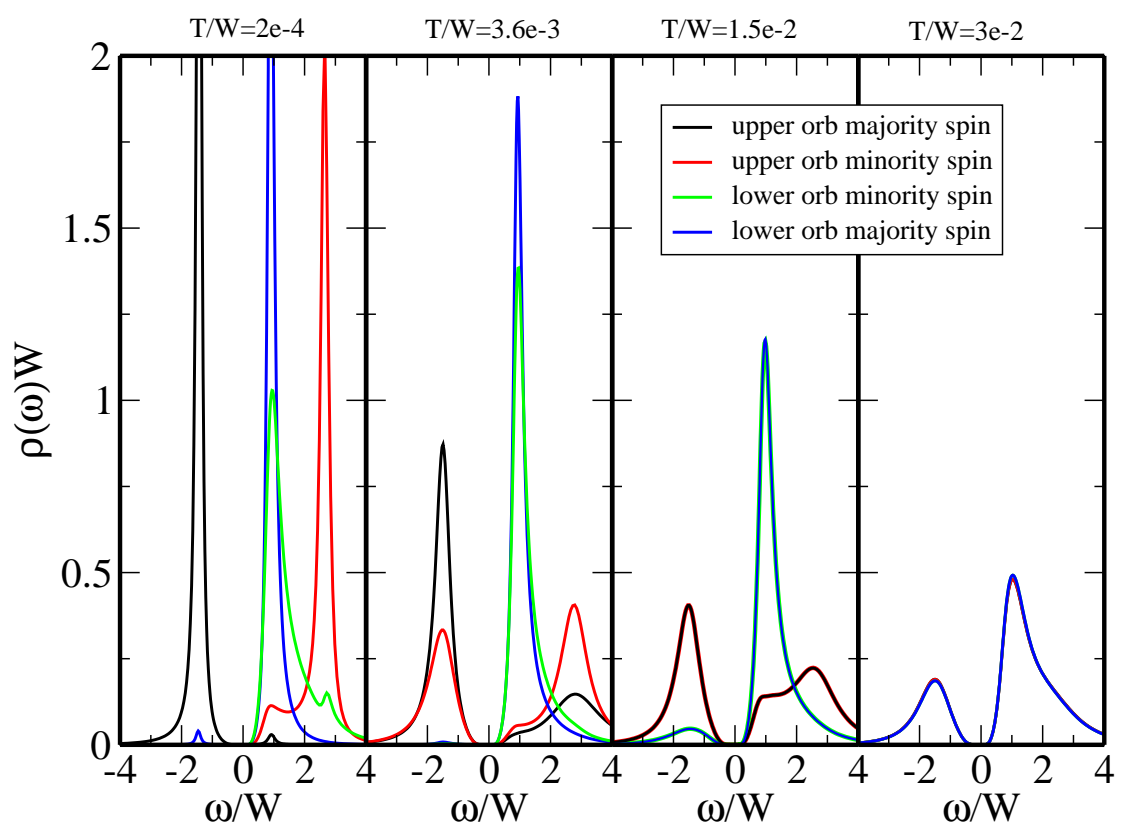

Figure 6.11: Temperature dependence of the orbitally ordered ferromagnetic state for $J / W=0.5$ and $U^{\prime} / W=3$ for four different temperatures. For $T / W=1.5 \cdot 10^{-2}$, both spin directions for each orbital are degenerate, denoting an orbitally ordered paramagnet. For $T / W=3 \cdot 10^{-2}$, all four lines lie above each other.

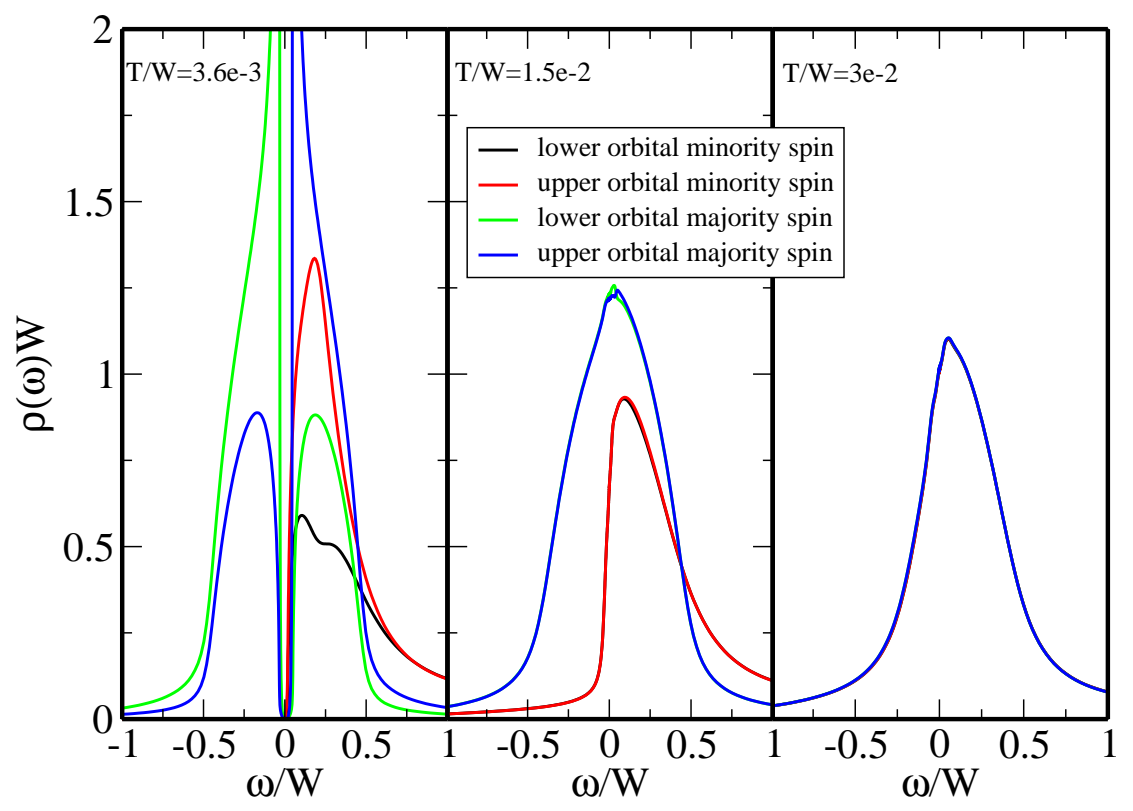

Figure 6.12: Temperature dependence of the orbitally ordered ferromagnetic state for $J / W=1$ and $U^{\prime} / W=1.45$ for three different temperatures. In the second panel, both orbitals are degenerate. In third panel, all lines lie above each other. 


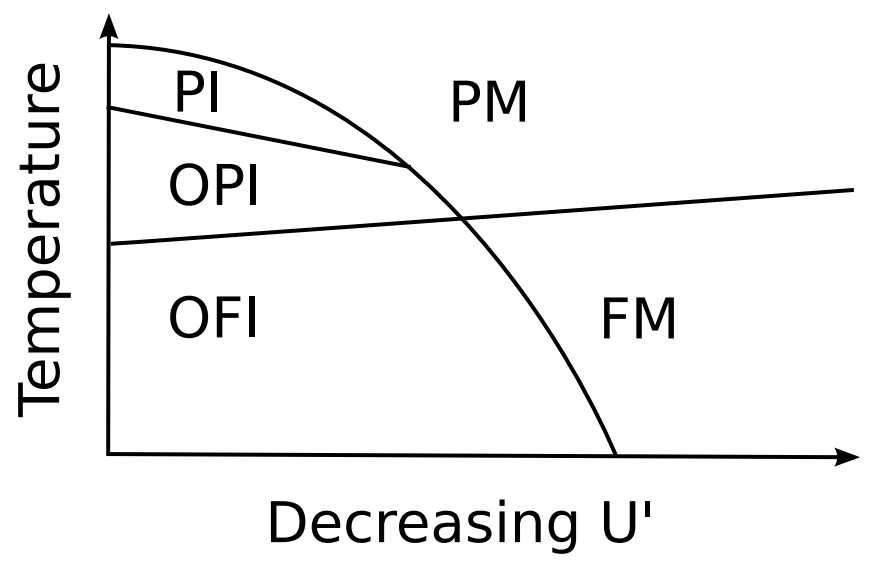

Figure 6.13: Proposed schematic $T-U^{\prime}$ phase diagram for quarter-filling. The following abbreviations are used: $\mathrm{PM}=$ paramagnetic meta; $\mathrm{PI}=$ paramagnetic insulator; OPI $=$ orbitally ordered paramagnetic insulator; $\mathrm{OFI}=$ orbitally ordered ferromagnetic insulator; $\mathrm{FM}=$ ferromagnetic metal.

the system is the fully polarized orbitally ordered ferromagnetic state, already shown above. When increasing the temperature, the ferromagnetic polarization vanishes, but the orbital order remains up to $T / W \approx 2 \cdot 10^{-2}$. Finally the orbital order becomes unstable towards a paramagnetic insulator. Obviously, the orbital order is the stronger ordering mechanism, persisting longer than the ferromagnetic order when increasing the temperature. But as the system remains in an insulating state, no temperature triggered ferromagnetic MIT can be observed in this parameter region. The insulating paramagnetic region reaches as high as $T / W \approx 0.4$. For a system with bandwidth $W=0.5 \mathrm{eV}$, this would imply a transition temperature of about $T \approx 2400 \mathrm{~K}$. Figure 6.12 shows the spectral functions in the orbitally ordered ferromagnetic phase near the ferromagnetic metal insulator transition for different temperatures. The orbital ordering now vanishes before the ferromagnetic transition occurs, and a ferromagnetic metallic state is stabilized. Finally, this state becomes unstable towards a paramagnetic metallic state. When increasing the temperature for a state in the homogeneous ferromagnetic phase, the ferromagnetic polarization vanishes and a paramagnetic metal is formed. Therefore, the transition temperatures of the ferromagnetic order and the orbital order change quite independently when varying the interaction parameters. Additionally, there has to be a paramagnetic MIT for high temperatures. Figure 6.13 shows a schematic phase diagram, which can be drawn after the just discussed results. Of course, the characteristics of the transition lines are here just proposed, but they include both temperature evolutions shown in the figures 6.11 and 6.12 . For high values of the inter-orbital density interaction $U^{\prime}$, there is the paramagnetic metal insulator transition. For lower temperatures a transition to the orbitally ordered paramagnet and the orbitally ordered ferromagnet can be found. In contrast, for inter-orbital density interaction $U^{\prime}$ close to the vanishing of the orbital order at $T=0$, one can see the temperature driven transition 

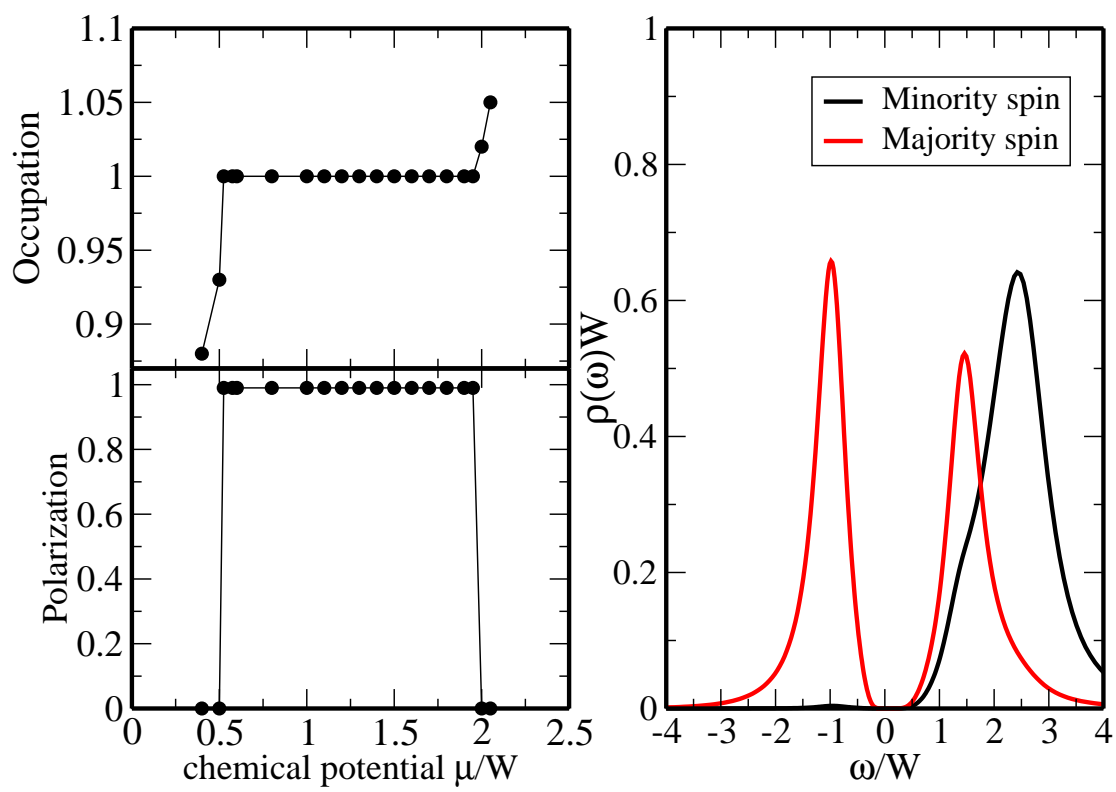

Figure 6.14: Antiferromagnetism for $J / W=0.5$ and $U^{\prime} / W=3$. The left panel shows the doping dependence of the occupation and polarization for the antiferromagnet state. The right panel shows the spectral function.

from the paramagnetic metal at high temperatures to the ferromagnetic metal and later eventually to the orbitally ordered ferromagnetic insulator.

\subsubsection{Antiferromagnetism and Charge Order}

In the region of large $U^{\prime}$, where the orbitally ordered ferromagnet is found, it was also possible to stabilize an antiferromagnetic Néel-phase at quarter-filling. As the system is dominated by ferromagnetic double exchange at quarter-filling, one actually does not expect such a phase here. An antiferromagnetic phase at quarter-filling was reported by T. Momoi [183] using an antiferromagnetic Hund's coupling. Like the orbitally ordered ferromagnet, this antiferromagnetic phase also exists only exactly at quarter-filling. The spectral function and the doping dependence can be seen in figure 6.14. The spectral function shows that also this state is a perfect insulator. When trying to dope it, one again finds phase separation to a paramagnetic metal away from quarter-filling. In order to find out which state is the thermodynamically stable one, I calculated the energy of both states. The result is that, as expected, the orbitally ordered ferromagnet has the lower energy, thus is the thermodynamically stable one. Nevertheless, the calculated energies are very small. Leaving out the chemical potential, which gives the same contribution for both states, the energy of the orbitally ordered ferromagnet is $\langle H\rangle=-0.0135 \mathrm{~W}$, and the energy of the antiferromagnetic state is $\langle H\rangle=-0.0085 \mathrm{~W}$. This is, compared to the chemical potential, which gives contributions of $\langle H\rangle \approx-W$, a very small splitting. Looking at the different terms in the energy, the kinetic energy gives a larger contribution for the antiferromagnetic state than for the ferromagnetic state, 


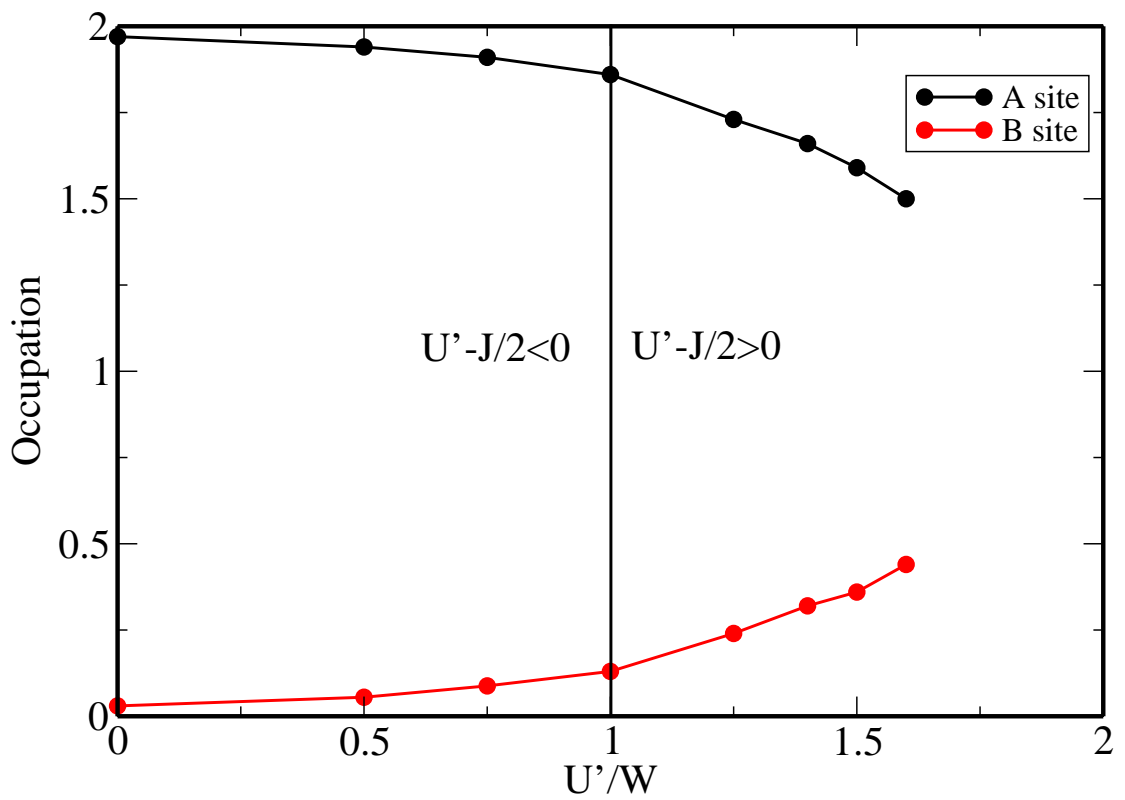

Figure 6.15: Occupation of neighboring sites in the charge ordered state for $J / W=2$. The inter-orbital interaction becomes repulsive for $U^{\prime} / W>1$. The lines are meant as guide to the eye.

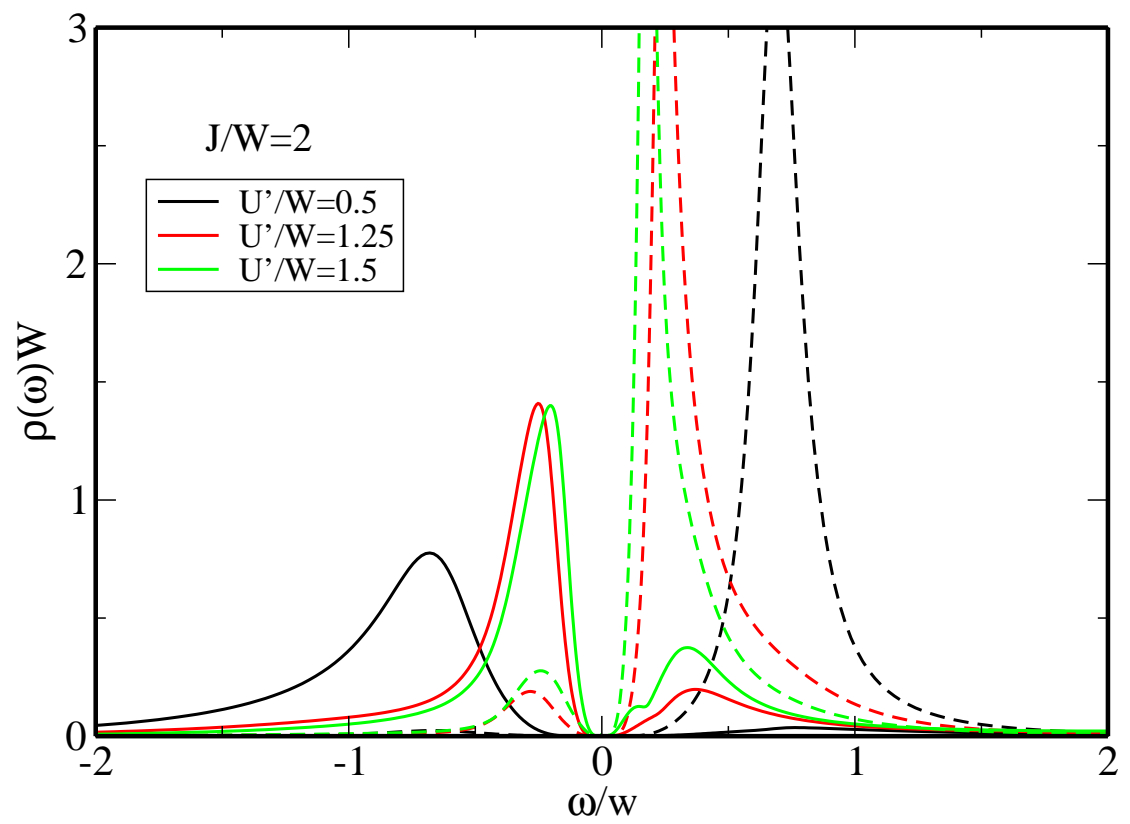

Figure 6.16: Spectral functions for the charge ordered state for $U / W=4, J / W=2$, and different $U^{\prime}$. The continuous (dashed) lines correspond to the occupied A-sites (nearly empty B-sites) 
while the interaction terms increase the energy of the antiferromagnet. Varying the parameters $J$ and $U^{\prime}$, I always find the antiferromagnetic state exhibiting the higher energy.

For large Hund's coupling $J / 2>U^{\prime}$ the inter-orbital density-density interaction becomes attractive. Although at a first glance such a large Hund's coupling appears unphysical, one can have situations, for example Jahn-Teller coupling to phonons, which can lead to additional contributions to $J$. In this case such an attractive interaction can effectively be generated and the physics will change dramatically. The first thing one notes is that it becomes very difficult to stabilize fillings other than $n=2$ or $n=0$. Inspired by this difficulty, I investigated charge ordered phases in this parameter regime. And indeed for sufficiently large Hund's coupling $J$ it is possible to stabilize a charge ordered state at quarter-filling, with alternating almost doubly occupied and nearly empty sites. The occupation of the different sites for this charge ordered phase can be seen in figure 6.15. The vertical line marks the point, where the inter-orbital density-density interaction changes from attractive in the left part to repulsive in the right part. Remarkably, even for repulsive density-density interaction, one can stabilize such charge ordered states. Of course, the occupation of neighboring sites approach each other. The end of the curves denote the point, at which it was impossible to stabilize this phase. From the two-orbital Anderson model one knows that the Kondo temperature [52] decreases exponentially and the doping dependence around quarter-filling becomes steeper for increasing Hund's coupling $J$. For an extended lattice this results in the charge ordered state, even for repulsive interaction $U^{\prime}-J / 2>0$. The spectral functions in this phase can be seen in figure 6.16. This charge density wave is a perfect insulator, too. The electron number from neighboring sites add up to two, which means that there is an average of one electron per site. When increasing $U^{\prime}$, the gap shrinks. Interestingly, the spectral function of the double occupied site possess an additional peak at $\omega / W \approx 5$. As the broadening of the NRG is very large in this regions, one cannot deduce the structure of these peaks or tell what is the origin of them.

\subsection{Summary}

This chapter dealt with the situation of two interacting degenerate bands. It is a basic model for an $e_{g}$-band, as it can be found in transition metal oxides with cubic symmetry. Like the one-orbital Hubbard model, the half-filled system is dominated by super exchange resulting in an antiferromagnetic phase. I especially looked at the interaction strength of $U / W=4$. For this interaction the system changes into an incommensurate spin density wave phase when doping away from half-filling.

In contrast to the one-orbital Hubbard model, the multi-orbital Hubbard model possesses a natural tendency towards ferromagnetism. For large enough Hund's coupling $J$ and density-density interactions $U$ and $U^{\prime}$ the system forms a homogeneous ferromagnetic state at $\langle n\rangle \approx 1.5$. Depending on the value of $J$ this phase can extend to occupations smaller than one. 
Quarter-filling is a very special point for the two-orbital Hubbard model. Besides a homogeneous ferromagnetic metal, one can stabilize an orbitally ordered ferromagnetic insulator for large $U^{\prime}$. Thus one can find a ferromagnetic metal insulator transition at quarter-filling. First studies in the temperature dependence of the phases at quarter-filling seem to indicate that also a paramagnetic metal insulator transition can be found. Such metal insulator transitions accompanied by a ferromagnetic phase transition provide the possibility to observe similar effects as the colossal magneto resistance effect in manganites. Therefore, the dependence of this MIT on temperature and magnetic field should be studied in more detail. Of course, in the manganites the just mentioned phenomenon occurs below quarter-filling and as a result of a transition between a ferromagnetic metal and a paramagnetic insulator, but the ingredients for this can already be found in this very simplified model. Including the neglected $t_{2 g^{-}}$ spin and the coupling to the lattice, one can expect to describe the manganites. But as the strengths of the neglected effects depend on the exact manganite compound, one can perhaps even hope that one can find a compound, which nearly shows the effects analyzed in this chapter.

Besides the just mentioned two ferromagnetic phases at quarter-filling, I additionally observed an antiferromagnetic insulator and an charge ordered insulator. The charge ordered state exists only for very strong Hund's coupling, which favors having two paired electrons at one site. In the same parameter region, in which the orbitally ordered ferromagnet exists, I found an antiferromagnetic insulator. But it seems as if this state has always the higher energy compared to the ferromagnetic state. 
CHAPTER 7

\section{Summary and Outlook}

This work dealt mainly with the topic of magnetic phases in the Hubbard model, which is a minimal model for analyzing the competition between localization induced by strong repulsive electron-electron interaction and delocalization induced by the electron hopping. This competition and the Pauli principle are the basic ingredients for magnetism as being observed in transition metal oxides. Additionally, the orbital degeneracy of the d-orbitals plays an important role in these materials. For better understanding the electronic properties in these materials, I have analyzed the influence of different hopping processes, the ratio between the interaction and the hopping, the filling of the lattice, and the degeneracy of the orbitals. Thus, I tried to identify the influence of each of the mentioned properties. For the purpose of calculating magnetic phases for the Bethe lattice I used the dynamical mean field theory (DMFT). Being exact in infinite dimensions it relates the lattice model to a quantum impurity model, which has to be calculated self-consistently.

\section{DMRG as an Impurity Solver}

Most of my results were gained using the numerical renormalization group (NRG), which is a well established method being able to calculate thermodynamic and spectral functions for a wide range of parameters. Additionally, I tested a newly developed code for the density matrix renormalization group (DMRG) as an impurity solver. The DMRG has the ability to perform ground state calculations for impurity models allowing for the calculation of ground state expectation values. For computing dynamical spectral functions, the DMRG uses an internal broadening, which results in a Lorentz convolution of the spectral function. Compared to the NRG, this broadening is much larger for frequencies near the Fermi energy, but smaller far away from it. Thus the 
DMRG is able to capture the behavior of the band edges and the substructures in the Hubbard bands, but eventually fails to correctly resolve the Kondo peak or small gaps at the Fermi energy. Unfortunately, this results in problems when using the DMRG as impurity solver within the DMFT. It can be shown that the half-filled one-orbital Hubbard model forms an antiferromagnetic insulator for every finite repulsive value of the Hubbard interaction $U>0$. This result can be reproduced by the NRG, but the DMRG fails if the interaction value is too small, resulting in a very small gap, which cannot be resolved anymore.

But the DMRG has a very big advantage to the NRG. For calculating dynamical properties properly within the NRG, one is limited to the one- or two-orbital case, because the local Fock space grows exponentially. Within the DMRG one can further split up the local Fock space, resulting in a linear growth of the chain length when increasing the orbital number. This is supposed to enable one to perform calculations for multi-orbital Hubbard models, perhaps even taking into account all 5 d-bands. Further studies on this subject should be done.

\section{One-Orbital Hubbard model}

In my thesis I showed many results on magnetic phases for the one- and twoorbital Hubbard model. The magnetic phase diagram of the one-orbital Hubbard model for a Bethe lattice with nearest neighbor hopping only is mainly covered by an antiferromagnetic insulating phase exactly at half-filling and a paramagnetic metallic phase away from half-filling. However, for large interaction strengths, there is an incommensurate spin density wave phase next to half-filling, which mainly stems from the antiferromagnetic fluctuation at halffilling. Interestingly, there is a transition from phase separation between the antiferromagnetic phase and the paramagnetic phase to this incommensurate phase exactly at the point, at which the paramagnetic metal insulator transition would occur. This stresses the connection between metal insulator transitions and magnetic phases. This incommensurate phase manifests itself in the DMFT as a non-convergent solution. I proposed here a way how to stabilize such phases for a Bethe lattice, but unfortunately the method seems to be rather unstable and unreliable for finding such solutions. In order to better understand such phases one should perform calculations for different lattices for which k-vectors can be defined, so one can look directly for spin density wave states.

Introducing a next nearest neighbor (NNN) hopping for the Bethe lattice changes the density of states, which becomes asymmetric and develops a singularity. This imposes frustration to the Néel-state at half-filling. It was argued that frustration helps pushing the metal insulator transition out of the antiferromagnetic dome. Increasing the frustration suppresses the Néel-temperature as well as the critical end point of the paramagnetic metal insulator transition. Only for NNN-hopping comparable in strength to the NN-hopping the paramagnetic metal insulator transition reaches out of the magnetic phases when increasing the temperature. This is the scenario seen in $\mathrm{V}_{2} \mathrm{O}_{3}$, but one still needs very large values of NNN-hopping. Additionally, one can observe that for such large NNN-hopping the Néel-state is replaced at half-filling by a spin 
density wave.

Surprisingly, for comparable NN- and NNN-hopping one can still stabilize an antiferromagnetic Néel-state away from half-filling. Thus frustration changes the filling at which a Néel-state is stable. Additionally, one can now find a ferromagnetic state, which is due to the singularity in the free density of states. Ferromagnetic states cannot be found for a Bethe lattice with semi-elliptic density of states. First introducing a singularity into the density of states makes ferromagnetic order possible. The ferromagnetic state reaches to the parameter regions, where the antiferromagnetic state can be stabilized, too. Comparing the energies of the ferromagnetic and the antiferromagnetic states in the fully frustrated model, there seems to be a phase transition between the ferromagnetic and the antiferromagnetic state. But the latter state has nearly equal energy to the paramagnetic state in this region. One could think of the possibility that in this parameter region even more magnetic states are stable due to the imposed frustration. But this is only a conjecture.

\section{Two-Orbital Hubbard model}

Finally, I showed results for the two-orbital Hubbard model describing correlations in a degenerate $e_{g}$-band. Close to half-filling, the model shows similar physics as the one-orbital Hubbard model. But away from half-filling, the twoorbital Hubbard model possesses even for symmetric free density of states the tendency towards ferromagnetism due to the degenerate orbitals. The most interesting point in the two-orbital Hubbard model is quarter-filling. There I could observe a clash of four different long range ordered phases. There is a phase transition between two different ferromagnetic states. One is a homogeneous metal, the other an orbitally ordered insulator. This phase transition can, for example, be triggered by changing the interaction parameters. Thus a metal insulator transition was found. Such transitions have always been very popular in the research of strongly correlated materials. The metal insulator transition observed here is strongly connected to the ferromagnetic phases observed at quarter-filling. It should be very interesting to study the influence of temperature and magnetic fields on this phase transition in more detail. If the transition point can be changed by applying a magnetic field, it would directly connect the resistivity to magnetic fields, which then can eventually be used for measuring magnetic fields. Very similar effects can be seen in manganites in the form of the colossal magneto resistance effect.

Besides these ferromagnetic states also antiferromagnetism and charge order could be found at quarter-filling. Charge order can especially be found for very large Hund's coupling, which favors states where two electrons are paired on one site.

This thesis explored some of the complexity, for which electronic correlations in an one- and two-orbital Hubbard model can be responsible. But mentioning the colossal magneto resistance effect in manganites, one should remember that the coupling to the very localized $t_{2 g}$-states and to the lattice is of great importance. Therefore, after studying the two-orbital Hubbard model, one should include these degrees of freedom step by step. By this one can analyze the effects for 
which each coupling is responsible and finally build up a very powerful model for manganites and other transition metal oxides. 


\section{Appendices}



APPENDIX A

\section{Calculation of the Ground State Energy}

In two parts of this thesis I calculate the kinetic energy of the DMFT solution. Especially in the case of the frustrated lattice, the integrals are numerically difficult, as singularities are involved. Therefore, parts of the integrals are performed analytically improving the accuracy. I will now shortly describe how one can treat the occurring integrals. The kinetic energy for $T=0$, see chapter 5.4.6, can be written as

$$
\begin{aligned}
\frac{\left\langle H_{T}\right\rangle}{N} & =\int_{-\infty}^{\infty} d \theta \epsilon(\theta) \rho(\theta) \int_{-\infty}^{0} d \omega\left(-\frac{1}{\pi}\right) \mathfrak{I m} \frac{1}{\omega+\mu-\epsilon(\theta)-\Sigma(\omega+i 0)} \\
\epsilon(\theta) & =t_{1} \theta+t_{2}\left(\theta^{2}-1\right) \\
\rho(\theta) & =\frac{1}{2 \pi} \sqrt{4-\theta^{2}} .
\end{aligned}
$$

As the self-energy is a calculated set of data points, the frequency integral has to be performed numerically. But the $\theta$-integral, can be performed exactly, in which the self-energy enters only as a complex parameter. The $\theta$-integral can be written as

$$
\int_{-2}^{2} d \theta\left(t_{1} \theta+t_{2}\left(\theta^{2}-1\right)\right) \frac{\sqrt{4-\theta^{2}}}{\zeta-\left(t_{1} \theta+t_{2}\left(\theta^{2}-1\right)\right)}
$$

where $\zeta=\omega+\mu-\Sigma(\omega+i 0)$. This integral can be solved by transforming it onto a complex contour by two consecutive substitutions. Firstly, writing 
$\theta=2 \cos (\phi)$ gives

$\int_{-\pi}^{0} d \phi 2 \sin (\phi)\left(2 t_{1} \cos (\phi)+t_{2}\left(4 \cos (\phi)^{2}-1\right)\right) \frac{2 \sin (\theta)}{\zeta-\left(2 t_{1} \cos \left(\theta+t_{2}\left(4 \cos (\theta)^{2}-1\right)\right)\right)}$

Secondly, one transforms this integral to the closed complex unit circle by substituting $\cos (\phi)=\frac{1}{2}(\exp (i \phi)+\exp (-i \phi))$ and $\sin =\frac{1}{2 i}(\exp (i \phi)-\exp (-i \phi))$ and using symmetry properties for performing the integral from $\int_{-\pi}^{\pi}$. Simultaneously one can write $z=\exp (i \phi)$ and $1 / z=\exp (-i \phi)$. The integral thus can be written as

$$
\frac{-1}{2 i} \oint_{S_{1}} \frac{1}{z} \frac{\left(z-\frac{1}{z}\right)^{2}\left(t_{1}\left(z+\frac{1}{z}\right)+t_{2}\left(\left(z+\frac{1}{z}\right)^{2}-1\right)\right)}{\zeta-\left(t_{1}\left(z+\frac{1}{z}\right)+t_{2}\left(\left(z+\frac{1}{z}\right)^{2}-1\right)\right)} .
$$

Sorting powers of $z$, this reads

$$
\frac{-1}{2 i} \oint_{S_{1}} \frac{1}{z^{3}} \frac{\left(z^{2}-1\right)^{2}\left(t_{1}\left(z^{3}+z\right)+t_{2}\left(\left(z^{2}+1\right)^{2}-z^{2}\right)\right)}{\zeta z^{2}-t_{1}\left(z^{3}+z\right)-t_{2}\left(\left(z^{2}+1\right)^{2}-z^{2}\right)} .
$$

The roots of the denominator polynomial can be found easily looking in equation (A.1). Thus, one is able to use the residue theorem to evaluate this integral for a given self-energy at frequency $\omega$. The remaining $\omega$-integration can then be performed numerically. 


\section{Bibliography}

[1] Stöhr J and Siegmann H, 2006 Magnetism From Fundamentals to Nanoscale Dynamics (Springer Verlag).

[2] Mattis D, 2006 The Theory of Magnetism Made Simple (World Scientific Pub Co).

[3] Gilbert W De Magnete, Magneticisque Corporibus, et de Magno Magnete Tellure.

[4] Jackson J, 1998 Classical Electrodynamics (Wiley\&Sons).

[5] Baym G, 1990 Lectures On Quantum Mechanics (Westview Press).

[6] Prinz G, 1998 Science 2821660.

[7] Fazekas P, 1999 Lecture Notes on Electron Correlations and Magnetism (World Scientific).

[8] Herring C, 1966 Magnetism, volume 4 (Academic Press NY).

[9] Solovyev I and Imada M, 2005 Phys. Rev. B 71045103.

[10] Maekawa S, Tohyama T, Barnes S, Ishihara S, Koshibae W, and Khaliullin G, 2004 Physics of Transition Metal Oxides (Springer).

[11] Jahn H and Teller E, 1937 Proc. R. Soc. London 161220.

[12] de Boer J and Verwey E, 1937 Proc. Phys. Soc. 4959.

[13] Mott N and Peierls R, 1937 Proc. Phys. Soc. 4972.

[14] Imada M, Fujimori A, and Tokura Y, 1998 Rev. Mod. Phys. 701039.

[15] McWhan D, Remeika J, Brinkman W, and Rice T, 1973 Phys. Rev. B 7 1920. 
[16] Katsufuji T, Taguchi Y, and Tokura Y, 1997 Phys. Rev. B 5610145.

[17] Miyasaka S, Okimoto Y, Iwama M, and Tokura Y, 2003 Phys. Rev. B 68 100406.

[18] Tokura Y, Taguchi Y, Okdada Y, Fujishima Y, Arima T, Kumagai K, and Iye Y, 1993 Phys. Rev. Lett. 702126.

[19] Terasaki I, Sasago K, and Uchinokura K, 1997 Phys. Rev. B 56 R12685.

[20] Mahan G, Sales B, and Sharp J, 1997 Physics Today 5042.

[21] Lawrence J, Riseborough P, and Parks R, 1981 Rep. Prog. Phys. 441.

[22] Thornton G, Morrison F, Partington S, Tofield B, and Williams D, 1988 J. Phys. C: Solid State Phys. 212871.

[23] Tokura Y, Okimoto Y, Yamaguchi S, Taniguchi H, Kimura T, and Takagi H, 1998 Phys. Rev. B 58 R1699.

[24] Bednorz K and Muller K, 1986 Z. Physik 64189.

[25] Lee P, Nagaosa N, and Wen X, 2006 Rev. Mod. Phys. 7817.

[26] Anderson P, 1987 Science 2351196.

[27] Bennemann K and Ketterson J, 2004 The Physics of Superconductors, volume 2 (Springer-Verlag).

[28] Coey J, Viret M, and Molnar S, 1999 Adv. Phys. 48167.

[29] Tokura Y (editor), 2000 Colossal Magnetoresistive Oxides (GORDON AND BREACH SCIENCE PUBLISHERS).

[30] Salamon M and Jaime M, 2001 Rev. Mod. Phys. 73583.

[31] Dagotto E, Hotta T, and Moreo A, 2001 Phys. Rep. 3441.

[32] Moshnyaga V, Sudheendra L, Lebedev L, Köster S, Gehrke K, Shapoval O, Belenchuk A, Damaschke B, Tendeloo G, and Samwer K, 2006 Phys. Rev. Lett. 97107205.

[33] Held K and Vollhardt D, 2000 Phys. Rev. Lett. 845168.

[34] Tokura Y, 2003 Physics Today 56(7) 50.

[35] Millis A, Littlewood P, and Schraiman B, 1995 Phys. Rev. Lett. 745144.

[36] Millis A, 1998 Nature 392147.

[37] Czycholl G, 2004 Theoretische Festkörperphysik (Springer Verlag).

[38] Hubbard J, 1963 Proc. R. Soc. A 238.

[39] Kanamori J, 1963 Prog. Theor. Phys. 275. 
[40] Gutzwiller M, 1963 Phys. Rev. Lett. 10159.

[41] Hohenberg P and Kohn W, 1964 Phys. Rev. 136 B864.

[42] Kohn W and Sham L, 1965 Phys. Rev. 140 A1133.

[43] Jones R and Gunnarsson O, 1989 Rev. Mod. Phys. 61689.

[44] Cox B, Coulthard M, and Lloyd P, 1974 J. Phys. F: Metal Phys. 4807.

[45] Gunnarsson O, Andersen O, Jepsen O, and Zaanen J, 1989 Phys. Rev. B 391708.

[46] Anisimov V and Gunnarsson O, 1991 Phys. Rev. B 437570.

[47] Nakamura K, Arita R, Yoshimoto Y, and Tsuneyuki S, 2006 Phys. Rev. B 74235113.

[48] Aryasetiawan F, Karlsson K, Jepsen O, and Schoenberger U, 2006 Phys. Rev. B $\mathbf{7 4} 125106$.

[49] Mosey N and Carter E, 2007 Phys. Rev. B 76155123.

[50] Hubbard J, 1964 Proc. R. Soc. A 277237.

[51] Oleś A, 1983 Phys. Rev. B 28327.

[52] Pruschke T and Bulla R, 2005 Eur. Phys. J. B 44217.

[53] Lieb E and Wu F, 1968 Phys. Rev. Lett. 201445.

[54] Baeriswyl D, Campbell D, Carmelo J, Guinea F, and Louis E, 1995 The Hubbard Model Its Physics and its Mathematical Physics (Springer).

[55] Tasaki H, 1998 J. Phys.:Condens. Matter 104353.

[56] Anderson P, 1950 Phys. Rev. 79350.

[57] Anderson P, 1959 Phys. Rev. 1152.

[58] Jonker G and Santen J, 1950 Physica 16337.

[59] Zener C, 1951 Phys. Rev. 82403.

[60] Anderson P and Hasegawa H, 1955 Phys. Rev. 100675.

[61] de Gennes P, 1960 Phys. Rev. 118141.

[62] Ising E, 1925 Z. Phys. 31253.

[63] Hazenwinkel M (editor), 2002 Encyclopedia of Mathematics (Ising model) (Springer).

[64] Onsager L, 1944 Phys. Rev. 65117.

[65] Scalapino D, 1986 J. Stat. Phys. 43757. 
[66] Scalapino D, 1995 J. Low Temp. Phys. 95169.

[67] Fehske H, Weisse A, and Schneider R (editors), 2008 Computational Many-Particle Physics, Lect. Notes Phys. 739 (Springer Verlag).

[68] Wiese U, 1993 Phys. Lett. B 311235.

[69] Chandrasekharan S and Wiese U, 1999 Phys. Rev. Lett. 833116.

[70] Georges A, Kotliar G, Krauth W, and Rozenberg M, 1996 Rev. Mod. Phys. 6813.

[71] Weiss P, 1907 J. Phys. (Paris) 6661.

[72] Brandt U and Mielsch C, 1989 Z. Phys. B 75365.

[73] Brandt U and Mielsch C, 1989 Z. Phys. B 79295.

[74] Brandt U and Mielsch C, 1991 Z. Phys. B 8237.

[75] Metzner W and Vollhardt D, 1989 Phys. Rev. Lett. 62324.

[76] Mueller-Hartmann E, 1989 Z. Phys. B 74507.

[77] Mueller-Hartmann E, 1989 Z. Phys. B 76211.

[78] Zlatic V and Horvatic B, 1990 Solid State Comm. 75263.

[79] Pruschke T, Jarrell M, and Freericks J, 1995 Adv. Phys. 44187.

[80] Georges A, 2004 AIP Conference Proceedings 7153.

[81] Orland H and Negele J, 1998 Quantum Many Particle Systems (Advanced Book Classics) (Westview Press).

[82] Fetter A and Walecka J, 2003 Quantum Theory of Many-Particle Systems (Dover).

[83] Kotliar G and Savrasov S, 2001 New theoretical approaches to strongly correlated systems (Kluwer Academics Publishers).

[84] Lichtenstein A, Katsnelson K, and Kotliar G, 2002 Electronic Correlations and Material Properties 2 (Kluwer NY).

[85] Kotliar G and Vollhardt D, 2004 Physics Today 5753.

[86] Keller G, Held K, Eyert V, Anisimov V, Byczuk K, Kollar M, Leonov I, Ren X, and Vollhardt D, 2006 NIC symposium, Realistic Modeling of Materials with Strongly Correlated Electrons.

[87] Held K, Nekrasov I, Keller G, Eyert V, Blümer N, McMahan A, Scalettar R, Pruschke T, Anisimov V, and Vollhardt D, 2006 Phys. stat. sol. b 243 2599 .

[88] Baxter R, 2008 Exactly Solved Models in Statistical Mechanics (Dover). 
[89] Economou E, 1979 Green's Functions in Quantum Physics (Springer Verlag).

[90] Kollar M, Eckstein M, Byczuk K, Blümer N, van Dongen P, de Cuba M, Metzner W, Tanaskovic D, Dobrosavljevic V, Kotliar G, and Vollhardt D, 2005 Ann. Phys. 14642.

[91] Eckstein M, Kollar, Byczuk K, and Vollhardt D, 2005 Phys. Rev. B 71 235119.

[92] Anderson P, 1961 Phys. Rev. 12441.

[93] Wilson K, 1975 Rev. Mod. Phys. 47773.

[94] Bulla R, Costi T, and Pruschke T, 2008 Rev. Mod. Phys. 80395.

[95] White S, 1992 Phys. Rev. Lett. 692863.

[96] Schollwoeck U, 2005 Rev. Mod. Phys. 77259.

[97] Kondo J, 1964 Progr. Theoret. Phys. (Kyoto) 3237.

[98] Hewson A, 1997 The Kondo Problem to Heavy Fermions (Cambridge University Press).

[99] Schrieffer J and Wolff P, 1966 Phys. Rev. 491.

[100] de Haas W W, de Boer J, and van den Berg G, 1934 Physica 1115.

[101] Krishna-murthy H, Wilkins J, and Wilson K, 1980 Phys. Rev. B 211003.

[102] Krishna-murthy H, Wilkins J, and Wilson K, 1980 Phys. Rev. B 211044.

[103] Bulla R, Pruschke T, and Hewson A, 1997 J. Phys.: Condens. Matter 9 10463.

[104] Press W, Teukolsky S, Vetterling W, and Flannery B, 2002 Numerical Recipes in $\mathrm{C}++$ Second Edition (Cambridge University Press).

[105] Sakai O, Shimizu Y, and Kasuya T, 1989 J. Phys. Soc. Jpn. 583666.

[106] Bulla R, Costi T, and Vollhardt D, 2001 Phys. Rev. B 64045103.

[107] Anders F and Schiller A, 2005 Phys. Rev. Lett. 95196801.

[108] Anders F and Schiller A, 2006 Phys. Rev. B 74245113.

[109] Peters R, Pruschke T, and Anders F, 2006 Phys. Rev. B 74245114.

[110] Weichselbaum A and von Delft J, 2007 Phys. Rev. Lett. 99076402.

[111] Hofstetter W, 2000 Phys. Rev. Lett. 851508.

[112] Bulla R, Hewson A, and Pruschke T, 1998 J. Phys.: Condens. Matter 10 8365 . 
[113] White S, 1993 Phys. Rev. B 4810345.

[114] Hallberg K, 2006 Adv. Phys. 55477.

[115] Nishimoto S and Jeckelmann E, 2004 J. Phys.: Condens. Matter 16613.

[116] Raas C, Uhrig G, and Anders F, 2004 Phys. Rev. B 69041102.

[117] Weichselbaum A, Verstraete F, Schollwoeck U, Cirac J, and von Delft J, 2008 arXiv:0504305v2 .

[118] Holzner A, Weichselbaum A, and von Delft J, 2008 arXiv:0804.0550v1 .

[119] White S and Noack R, 1992 Phys. Rev. Lett. 683487.

[120] Feynman R, 1998 Statistical Mechanics: A Set of Lectures (WESTVIEW PRESS).

[121] Bai Z, Demmel J, Dongarra J, Ruhe A, and van der Vorst H, 1987 Templates for the Solution of Algebraic Eigenvalue Problems: A Practical Guide (Society for Industrial Mathematics).

[122] Golub G, 1996 Matrix Computations (Johns Hopkins).

[123] White S, 1998 Phys. Rep. 301187.

[124] Gaite J, 2001 Mod. Phys. Lett. A 161109.

[125] Hallberg K, 1995 Phys. Rev. B 52 R9827.

[126] Kuehner T and White S, 1999 Phys. Rev. B 60335.

[127] Pati S, Ramasesha S, Shuai Z, and Bredas J, 1999 Phys. Rev. B 5914827.

[128] Jeckelmann E, 2002 Phys. Rev. B 66045114.

[129] Raas C, 2005 Dynamic Density-Matrix Renormalization for the Symmetric Single Impurity Anderson Model (Ph.D. Thesis Koeln).

[130] Jaynes E, 1985 in Maximum-Entropy and Bayesian Methods in inverse Problems (Dordrecht: Reidel).

[131] Skilling J and Gull S, 1985 in Maximum-Entropy and Bayesian Methods in inverse Problems (Dordrecht: Reidel).

[132] Narayan R and Nityananda R, 1986 Ann. Rev. Astron. Astrophys. 24127.

[133] Dargel P, 2009 Local Density of States of the Single Impurity Anderson Model (Diploma Thesis Goettingen).

[134] Zitko R and Pruschke T, 2009 Phys. Rev. B 79085106.

[135] Nishimoto S, Pruschke T, and Noack R, 2006 J. Phys.: Condens. Matter 18981. 
[136] Peters R and Pruschke T, 2009 Phys. Rev. B 79045108.

[137] Peters R and Pruschke T, 2009 New J. Phys. 11083022.

[138] Bulla R, 1999 Phys. Rev. Lett. 83136.

[139] Rozenberg M, Chitra R, and Kotliar G, 1999 Phys. Rev. Lett. 833498.

[140] Georges A and Krauth W, 1993 Phys. Rev. B 487167.

[141] Rozenberg M, Kotliar G, and Zhang X, 1994 Phys. Rev. B 4910181.

[142] van Dongen P, 1994 Phys. Rev. Lett. 74182.

[143] van Dongen P, 1995 Phys. Rev. B 541584.

[144] Zitzler R, Pruschke T, and Bulla R, 2002 Eur. Phys. J. B 27473.

[145] Freericks J and Jarrell M, 1995 Phys. Rev. Lett. 74186.

[146] Jarrell M, 1992 Phys. Rev. Lett. 69168.

[147] Zitzler R, Tong, Pruschke T, and Bulla R, 2004 Phys. Rev. Lett. 93 016406.

[148] Rozenberg M, Kotliar G, Kajueter H, Thomas G, Rapkine D, Honig J, and Metcalf P, 1995 Phys. Rev. Lett. 75105.

[149] Nagaoka Y, 1966 Phys. Rev. 147392.

[150] Obermeier T, Pruschke T, and Keller J, 1997 Phys. Rev. B 568479.

[151] Park H, Haule K, Marianetti C, and Kotliar G, 2008 Phys. Rev. B 77 035107.

[152] Žitko R, 2009 arXiv:0908.0613.

[153] Dagotto E, 1994 Rev. Mod. Phys. 66763.

[154] Arrigoni E and Strinati G, 1991 Phys. Rev. B 447455.

[155] Fleck M, Lichtenstein A, Oleś A, Hedin L, and Anisimov V, 1998 Phys. Rev. Lett. 802393.

[156] Fleck M, Lichtenstein A, Oleś A, and Hedin L, 1999 Phys. Rev. B 60 5224 .

[157] Peters R and Pruschke T, 2007 Phys. Rev. B 76245101.

[158] Nishimoto S, Gebhard F, and Jeckelmann E, 2004 J. Phys.: Condens. Matter 167063.

[159] Karski M, Raas C, and Uhrig G, 2005 Phys. Rev. B 72113110.

[160] Karski M, Raas C, and Uhrig G, 2008 Phys. Rev. B 77075116. 
[161] Shraiman B and Siggia E, 1988 Phys. Rev. Lett. 60740.

[162] Dagotto E, Joynt R, Moreo A, Bacci S, and Gagliano E, 1990 Phys. Rev. B 419049.

[163] Obermeier T, Pruschke T, and Keller J, 1996 Ann. Physik 5137.

[164] Sangiovanni G, Toschi A, Koch E, Held K, Capone M, Castellani C, Gunnarsson O, Mo S, Allen J, Kim H, Sekiyama A, Yamasaki A, Suga S, and Metcalf P, 2006 Phys. Rev. B 73205121.

[165] Duffy M and Moreo A, 1997 Phys. Rev. B 55 R676.

[166] Hofstetter W and Vollhardt D, 1998 Ann. Phys. 748.

[167] Chitra R and Kotliar G, 1999 Phys. Rev. Lett. 832386.

[168] Eckstein M, Kollar M, Potthoff M, and Vollhardt D, 2007 Phys. Rev. B 75125103.

[169] Pothoff M, 2003 Eur. Phys. J. B 32429.

[170] Aspelmeier T, 2009 private communication .

[171] Stoner E, 1938 Proc. R. Soc. A 165372.

[172] Mielke A, 1991 J. Phys. A 24 L73.

[173] Tasaki H, 1992 Phys. Rev. Lett. 691608.

[174] Tasaki H, 1998 Prog. theor. Phys. 99489.

[175] Wahle J, Blümer N, Schlipf J, Held K, and Vollhardt D, 1998 Phys. Rev. B 5812749 .

[176] Ulmke M, 1998 Eur. Phys. J. B 1301.

[177] Arita R, Onoda S, Kuroki K, and Aoki H, 2000 J. Phys. Soc. Jpn. 69 1181.

[178] Pandey S and Singh A, 2007 Phys. Rev. B 75064412.

[179] Tokura Y and Nagaosa N, 2000 Science 21462.

[180] Peters R and Pruschke T, 2009 arXiv:0908.3990 .

[181] Fresard R and Kotliar G, 1997 Phys. Rev. B 5612909.

[182] Held K and Vollhardt D, 1998 Eur. J. Phys. B 5473.

[183] Momoi T and Kubo K, 1998 Phys. Rev. B 58 R567.

[184] Didkuh L, Hankevych V, Kramar O, , and Skorenkyy Y, 2002 Condens. Matter 14827. 
[185] Fresard R, Raczkowski M, and Oles A, 2005 Phys. Stat. Sol. B 242370.

[186] Sakai S, Arita R, and Aoki H, 2007 Phys. Rev. Lett. 99216402.

[187] Kubo K, 2009 Phys. Rev. B 79 020407(R).

[188] Roth C, 1966 Phys. Rev. 149306.

[189] Kugel K and Khomskii D, 1973 Sov. Phys. JETP 37725.

[190] Cyrot M and Lyon-Caen C, 1973 J. Phys. C: Solid State Phys. 6 L247.

[191] Lu F, Chen D, and Zou L, 2009 Chinese Phys. Lett. 26097501. 


\section{Thanks}

Finally, I want to say "THANK YOU".

First, I want to thank my adviser Thomas Pruschke. He has always an open door for questions. It was a pleasure and honor working, drinking coffee, and visiting temples with him. I am very grateful for his support, trust, and friendship.

I also want to thank Frithjof Anders for agreeing to review this thesis. Additionally, it is always enlightening to discuss with him on conferences.

At this point I want to thank my family for their interest and support during the last years.

I thank all the people, who read parts of this thesis and gave me some good advice: Andreas, Katharina, Ludger, Oliver, Piet, Sebastian, Sigrun, and Till. Especially, I thank Andreas, who also has always an open door for questions. Additionally, I thank Ansgar, Anton, Piet, and Till for playing some good games table-soccer with me, and Riccardo for all the good Pizza and Coffee.

During my thesis, I was allowed to visit Kyoto for nearly three months. For giving me this opportunity, I give thanks to Kawakami-sensei, GCOE, and Pruschke-sensei. I also want to thank Koyama-san and Noda-san for their friendship and support in Kyoto. 



\section{Lebenslauf}

Persönliche Daten

Name, Vorname

Anschrift

Mail

Staatsangehörigkeit

Geburtstag

Geburtsort

Werdegang

1988-2000

2000-2001

2001-2003

2003-2006

2006-2009

Kenntnisse

Sprachen
Peters, Robert

Im Hassel 13, 37077 Göttingen

peters@theorie.physik.uni-goettingen.de

Deutsch

15. Dezember 1981

Karl-Marx-Stadt (heute Chemnitz)

Grundschule und Gymnasium in Chemnitz

Abschluss: Abitur 2000

Wehrpflicht in Cham und Regensburg

Grundstudium der Physik an der TU Chemnitz

Studium der Physik an der Universität Göttingen

Abschluss: Physikdiplom 2006

Promotion in Physik an der Universität Göttingen

Englisch, Japanisch (Grundkenntnisse) 